UNIVERSIDAD POLITÉCNICA DE MADRID

Escuela Técnica Superior de Ingenieros Industriales

\title{
Modelling ACTIVIties OF DAILY LIVING APPLIED TO COGNITIVE REHABILITATION
}

\section{DOCTOR OF PHILOSOPHY}

AUTOMATION AND ROBOTICS

Javier I. Rojo Lacal

Ingeniero en Automática y Electrónica Industrial 

DEPARTAMENTO DE AUTOMÁTICA, INGENIERÍA ELÉCTRICA, ELECTRÓNICA E INFORMÁTICA INDUSTRIAL

Escuela Técnica Superior de Ingenieros Industriales

\title{
Modelling ACTIVITIES OF DAILY LIVING APPLIED TO COGNITIVE REHABILITATION
}

\author{
DOCTOR OF PHILOSOPHY \\ AUTOMATION AND ROBOTICS
}

Author: Javier I. Rojo Lacal

Ingeniero en Automática y Electrónica Industrial

Advisor: Manuel Ferre Pérez

Doctor Ingeniero Industrial 



\section{UNIVERSIDAD POLITÉCNICA DE MADRID}

Escuela Técnica Superior de Ingenieros Industriales

\section{Modelling ACtivities OF DAILY LiVing APPLIED TO COGNITIVE REHABILITATION}

Autor: Javier I. Rojo Lacal

$\underline{\text { Tribunal: }}$

Presidente: $\quad$ Dra. M ${ }^{\mathrm{a}}$ Teresa Arredondo

Secretario Dr. Claudio Rossi

Vocal A : $\quad$ Dr. Antonio Giménez

Vocal B : $\quad$ Dr. José Mª Azorín

Vocal C : $\quad$ Dr. Christos Giachritsis

Suplente A : Dra. Concepción Alicia Monje

Suplente B : $\quad$ Dr. Raúl Marín

Acuerdan otorgar la calificación de: 



\section{AgRAdecimientos}

Hace tiempo que decidí embarcarme en esta aventura y es imposible evitar mirar atrás y hacer balance de lo acontecido en este tiempo. Si de todas las personas que han desfilado por mi vida tuviera que nombrar a la precursora de todo esto, esa persona sería sin duda D. Ramón Galán. Todos los éxitos y la motivación de esta tesis parten de una visita trivial a un departamento de robótica y son responsabilidad directa tuya.

Tampoco me olvido del día en el que Rafael Aracil y Manuel Ferre se acercaron al puesto en el que trabajaba y me ofrecían participar en algo que a posteriori supuso un giro a mi vida. A ellos también les agradezco haber llegado a mi actual situación. Desde entonces hasta ahora sólo puedo valorar la suerte de haber podido aprender y formarme en aquello que es mi vocación. Gracias por aportarme tanto. Siempre sabré valorar la oportunidad recibida. De mi director, Manuel Ferre, siempre agradeceré su eterna paciencia y tolerancia.

Quiero agradecer a todos los compañeros que han pasado por el departamento de Robots y Máquinas Inteligentes (ROMIN). Todos y cada uno de ellos me enseñaron algo. Gracias a todos los Ronin, en especial a: Jordi Barrio, Nuria Moratalaya, Sandra Campo, Ignacio Galiana, José Breñosa, José M. Cogollor, Alex Owen-Hill, Prithvi Pagala, Ramviyas Parasunam, Elena García, Francisco Suárez, Santiago Martínez, Miguel Gómez, Arturo Colina, Carlos Gutiérrez, Luis Rubio, José Luis Samper, Sofía Coloma, Joaquín Macanás, Gonzalo García, Juan Carlos Ramos y Doris Bautista. Ha sido un placer conoceros y convivir con vosotros entre el horizonte de sucesos y la repostería más amarga.

Este trabajo no habría sido posible tampoco sin la colaboración de todas y cada una de las personas que formaron parte del desarrollo del proyecto CogWatch. Quiero agradecer a cada uno de los compañeros que participaron en este proyecto ya que, sin la cooperación y estrecha colaboración que ofrecieron, nunca habría llegado hasta este punto. A partir de vuestro trabajo cobran sentido las famosas palabras de Bernard de Chartres y es que, sin vosotros, nunca podría haberme planteado el reto. Gracias a todos y cada una de las personas que formasteis parte del proyecto por aportar diferentes puntos de vista y soluciones, he aprendido mucho de todos. 
Nunca olvidaré los meses que pude disfrutar en Munich, donde conocí a gente maravillosa. Gracias al profesor Joachim Hermsdörfer por permitirme, durante mi estancia, ser uno más en su departamento y ser testigo de su día a día y buen hacer. ¡No se puede ser más acogedor!, debo dar las gracias en especial a Elvira Salazar, a Marta Bienkewich y a Kerstin Laimgruber por toda su ayuda y consejos para instalarme en Munich. Aprendí y disfruté mucho con vosotros y quiero darles las gracias, Yi-Huang, Marie-Luise, MaFe, "Bro" J, Philipp, Saskia, Alan y Ferdinand. Os agradezco a todos vosotros los buenos momentos vividos en Munich, nunca olvidaré ese picnic en Walchensee.

Quiero tener también una mención especial a María Teresa Arredondo y su grupo Life Supporting Technologies, por haberme permitido seguir adelante con el trabajo aquí iniciado, qué es mi actual pasión. Y por supuesto a Matteo Pastorino y Alessio Fioravanti por ser tan buenos compañeros, por los buenos momentos vividos y el apoyo dado durante los momentos menos buenos. Es de recibo agradecerles, también, que me enseñaran, por descuido, algo de italiano, Butta n'...!

En un terreno más personal, quiero dar las gracias a las personas que más me han apoyado, empezando por mis amigos.

A mi familia por su continuo apoyo. La dedicación y las ganas nunca me han faltado gracias a la ilusión y actitud que desde pequeño me inculcaron las personas que más me han dado en esta vida, mis padres; Lourdes y Javier.

A mi cuñado Javier y a mi hermana Patricia y a ese pequeño regalo en forma de bichejo que se está formando y que pronto colmaremos de cariño.

A la familia de mi novia por hacerme sentir tan querido y agasajado, gracias Maite, Luis y Álvaro.

Por último quiero agradecer a la persona que siempre ha estado ahí, por sus consejos, su apoyo incondicional y por ser la persona que da sentido a mi vida, gracias Maite. 


\section{ACKNOWLEDGMENTS}

Some time ago I decided to embark on this adventure and it is impossible to avoid looking back and take stock of what happened at this time. From all the people who have passed through my life if I had to name the forerunner of all this, that person would certainly be D. Ramón Galán. All successes and motivation of this thesis are based on a trivial visit to a department of robotics and direct responsibility is yours.

Nor do I forget the day when Rafael Aracil and Manuel Ferre came to my sitting place where I was working and offered me to participate in something that, post, was a turnabout in my life. I also thank you for having come to my current situation. From then until now I can only assess fortunate to have been able to learn and grown in my vocation. Thank you for bring me so much, I will always know appreciate the opportunity received. From my director, Manuel Ferre, I always appreciate your eternal patience and tolerance.

I want to thank all colleagues who have gone through the Department of Robots and Intelligent Machines (ROMIN). Each and every one of them taught me something. Thanks to all Ronin, especially Jordi Barrio, Nuria Moratalaya, Sandra Campo, Ignacio Galiana, José Breñosa, José M. Cogollor, Alex Owen-Hill, Prithvi Pagala, Ramviyas Parasunam, Elena Garcia, Francisco Suarez, Santiago Martinez, Miguel Gomez, Arturo Colina, Carlos Gutierrez, Luis Rubio, Jose Luis Samper, Sophia Coloma, Joaquín Macanás, Gonzalo Garcia, Juan Carlos Ramos and Doris Bautista. It has been a pleasure to meet you and live with you between the event horizon and the bitterest backery.

This work would not have been possible either without the collaboration of each and every one of the people who were part of the development of CogWatch project. I want to thank each of the partners who participated in this project because, without the cooperation and close cooperation offered would never have come this far. From your work make sense the famous words of Bernard of Chartres and it is a fact that, without you, I could never have raised this challenge. Thanks to each and every one of the people who were formed part of the project. They provided different viewpoints and solutions, I have learned a lot from everyone.

I never forget the months I could enjoy in Munich, where I met wonderful people. Thanks to Professor Joachim Hermsdörfer for allowing me during my stay, to be one 
more in his department and witness their daily lives and good work. No one can be more welcoming!, I must thank especially Elvira Salazar, Martha Bienkewich and Kerstin Laimgruber for all your help and advice to settle in Munich. I learned and enjoyed meeting with you and want to thank Yi-Huang, Marie-Luise, MaFe, "Bro" J, Philipp, Saskia, Alan and Ferdinand. I thank you all the good times in Munich, I will never forget the picnic in Walchensee.

I would also have a special mention to Maria Teresa Arredondo and her group Life Supporting Technologies, for allowing me to go ahead with the work started here, what is my current passion. And of course Matteo and Alessio Pastorino Fioravanti for being so good companions for the good times and the support given during less good moments. Thank receipt is also to teach me, inadvertently, some Italian, Butta n '...!

On a more personal level, I want to thank the people who have supported me over, starting with my friends.

To my family for their continuous support. Dedication and work I have never missed thanks to the enthusiasm and attitude instilled in me since childhood from the people who have given me more in this life, my parents; Lourdes and Javier.

To my brother in law Javier and my sister Patricia and that little gift in the form of "bichejo" being formed and who we soon overwhelm of affection.

To the family of my girlfriend for making me feel so loved and feted, thanks Maite, Luis and Alvaro.

Finally I want to thank the person who has always been there, for their advice, their unconditional support and for being the person who gives meaning to my life, thanks Maite. 


\section{RESUMEN}

El modelado de tareas describe las actividades que se deben realizar a fin de alcanzar un objetivo. Para que el modelo sea completo se deben contemplar las diferentes formas de alcanzar dicho objetivo dentro del modelo. El modelado de tareas se ha constituido como un campo de estudio con un amplio conjunto de aplicaciones. Estas aplicaciones nacen de la observación y tienen como finalidad mejorar la eficiencia del sistema en el que se aplican.

Se pueden enunciar los tres grandes campos de aplicación del modelado de tareas. Estos campos son la monitorización, el diseño y, principalmente, la toma de decisiones. En esta tesis, en particular, se ha realizado una revisión en el marco de la rehabilitación, en concreto, la rehabilitación cognitiva.

En primer lugar, se ha realizado una reseña del estado actual del modelado de actividades, a partir de ella se busca profundizar en las ventajas que presenta el modelado de tareas y sus posibles aplicaciones. Posteriormente se indaga brevemente en los primeros estudios que se realizaron para modelar la actividad humana. A partir de estos primeros estudios se busca conocer las motivaciones intrínsecas que suscitan aplicar el modelado. A continuación se detallan las técnicas más interesantes para aplicar un modelo que actúe como crítico de las acciones realizadas por un actor. Este modelo deriva del famoso modelo sugerido por los padres del aprendizaje por refuerzo, Sutton y Barto, y los modelos que se derivaron de su estudio.

Por otro lado, es preciso detallar el papel de la rehabilitación cognitiva en la actualidad y las posibles consecuencias derivadas de padecer un accidente cerebro-vascular. El papel de la tecnología para paliar los efectos que puede tener en el día a día de las personas que padecen secuelas de un ictus es tratado con un enfoque novedoso. Para entender la importancia del marco de esta tesis, se presenta varias de las principales enfermedades cognitivas asociadas al ictus y se introducen las consecuencias que tienen en la ejecución de tareas de la vida diaria. 
A continuación, se plantea un modelo para la toma de decisiones que actúa como núcleo de un sistema de asistencia para la realización de tareas. A partir de una pre-evaluación del sistema se detectan las carencias de aplicar una estrategia simple y se muestra una posible solución a partir de un sistema supervisor basado en el contraste del modelo proponiendo un sistema distribuido más efectivo que el inicial.

Posteriormente, se analiza la aplicación de este modelo al guiado y se presenta una interfaz multimodal efectiva para guiar a un usuario a través de tareas secuenciales. Se destacan los criterios y funcionalidades necesarias para dicha aplicación. Un pequeño experimento permite comprobar la efectividad de la aplicación para su finalidad principal, el aprendizaje.

La monitorización del usuario también ha sido analizada. Tres aspectos han sido contemplados, el seguimiento supervisado de tareas a partir de técnicas mínimamente invasivas; un sensor de profundidad de bajo coste basado en infrarrojos, el tiempo de reacción a estímulos del usuario; se demuestra la utilidad de una herramienta dedicada, diseñada para dicho propósito, frente a programas existentes no dedicados y, por último, un sistema de medida de la presión arterial para estimar diversos parámetros fisiológicos del usuario.

Por último, el modelo descrito es implementado y evaluado dentro de una plataforma más compleja dedicada a la rehabilitación cognitiva. Esta herramienta busca facilitar la rehabilitación del paciente en tareas cotidianas dentro del entorno doméstico. A partir de las valoraciones de clínicos y pacientes y de diversos test para determinar la eficacia de la herramienta se concluye que el modelo utilizado tiene una gran utilidad.

La conclusión de esta tesis es que el modelado de tareas permite mejoras cualitativas en los sistemas que requieren alguna de sus tres principales áreas de aplicación previamente citadas. En particular, se ha demostrado su efectividad aplicada a tareas de rehabilitación cognitiva. Cómo trabajos futuros se plantea la utilización del modelo en otros campos de aplicación y se comentan brevemente las líneas de investigación actuales que auguran un escenario futuro prometedor para la aplicación de las técnicas presentadas. 


\section{ABstract}

A task model describes the activities to be performed to achieve a goal, and the different ways to achieve the target. Modelling has been established as a field of study with a large set of applications. These applications came from observation and are intended to improve the efficiency of the system in which it is applied.

It can be stated that the three major fields of application of tasks modelling are monitoring, design and, mainly, decision making. This thesis has been focussed on a review within the framework of rehabilitation with special attention in cognitive rehabilitation.

First, a review of the current status of modelling activities, in order to deep in the advantages of modelling tasks and their possible applications is introduced. Then, this thesis briefly explores the first studies which were performed to model human activity. From the report of these early studies it is intended to understand the intrinsic motivations hereby aroused applied modelling. Up to this point, the most interesting techniques are described for applying a model to act as critic of the actions taken by an actor. This model is derived from the famous model suggested by the parents of reinforcement learning, Sutton and Barto, and the models derived from their study.

On the other hand, it is necessary to detail the role of cognitive rehabilitation at present and some of the possible consequences of suffering a cerebrovascular accident. The role of technology to mitigate the effects and sequelae of stroke on the daily lives of people is treated with a novel approach. To understand the importance of the framework of this thesis, a summary of the main cognitive disorders is presented and the implications in performing daily activities are mentioned.

Following the description of the problematic which aims this study, a decision-making model to act as the core of an assistive system, while performing tasks, is proposed. From a pre-evaluation of the system deficiencies of applying a simple strategy based on markovian methods are 
detected and, consequently, a possible solution from a supervisory system based on model checking is presented. It is concluded that a distributed system is more effective than the initial system in this context.

Subsequently, the application of this model for guidance is analysed. An effective multimodal interface for guiding the user through sequential tasks is described. To evaluate the reaction time to stimuli, the usefulness of a dedicated tool, designed for this purpose, is reported, the designed system is compared with existing programs. User monitoring has also been briefly analysed from minimally invasive techniques; a low cost infrared-based depth sensor and a blood pressure module.

Finally the model described is implemented and evaluated in a more complex platform dedicated to cognitive rehabilitation. This tool aims to facilitate the rehabilitation of the patient in everyday tasks in the home environment. From clinical and patient assessments and various test to determine the effectiveness of the tool it is concluded that the model presented is effective for its' purpose.

The possibility of future work looking to expand the fields of application of the model and to improve the techniques presented are discussed. To that end, some guidelines have been suggested. 


\section{Contents}

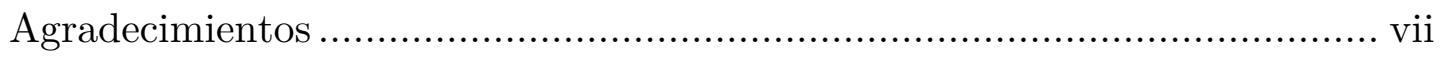

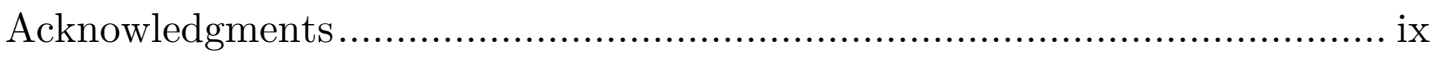

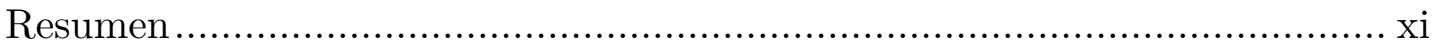

Abstract f...................................................................................... xii

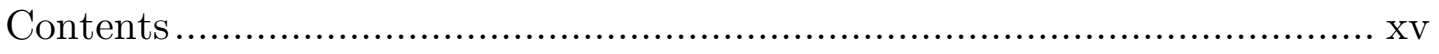

Acronyms and Abbreviations ........................................................... xxi

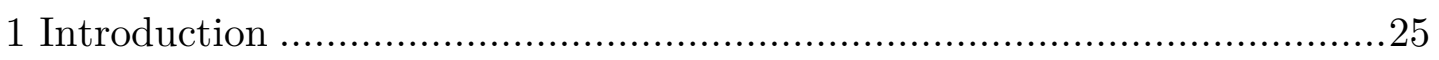

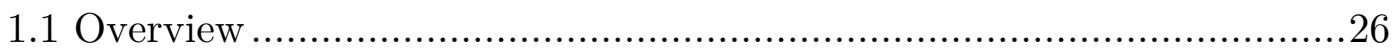

1.2 Research motivation.................................................................27

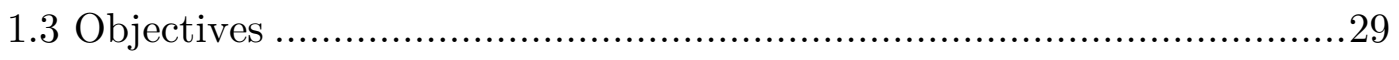

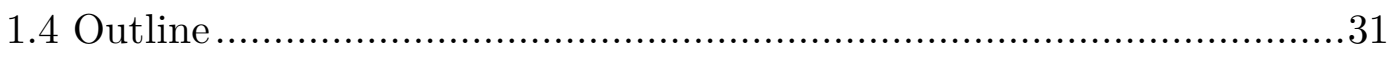

2 State of the Art on task modelling and post-stroke rehabilitation .............33

2.1 Cognitive disorders following a stroke........................................... 33

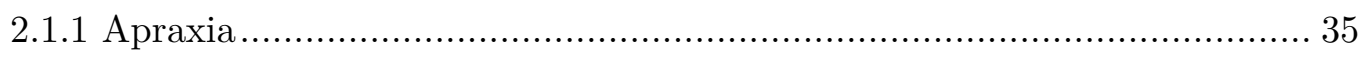

2.1.2 Action Disorganization Syndrome ….............................................. 38

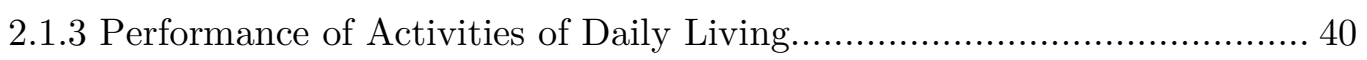

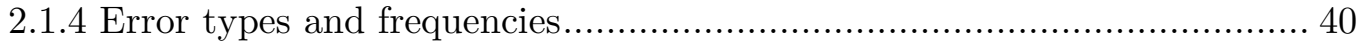

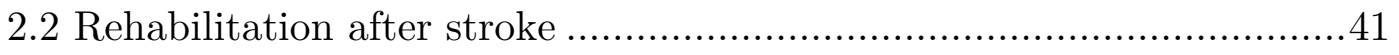

2.3 Gathering information from the expert .................................. 45

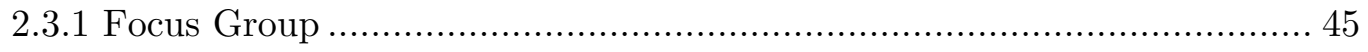

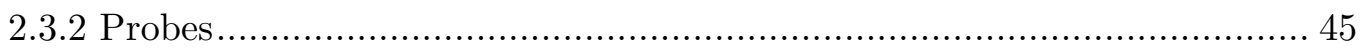

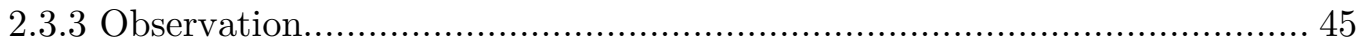

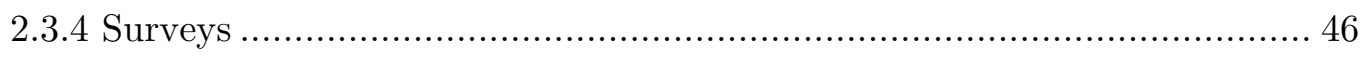

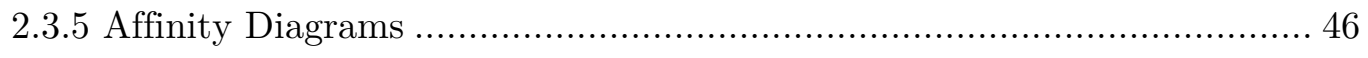




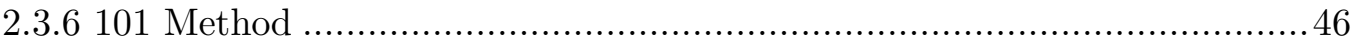

2.4 The goal of modelling .............................................................. 47

2.5 Dimensionality of Task Modelling ................................................. 48

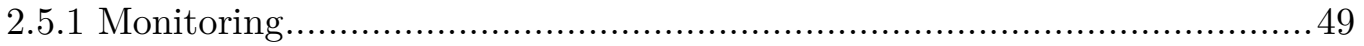

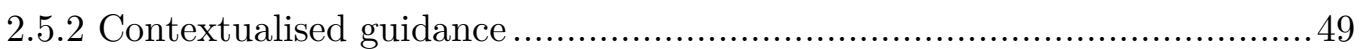

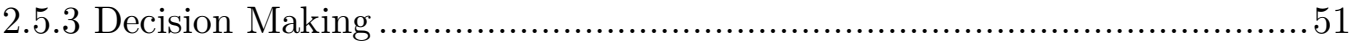

2.6 The study of actions............................................................... 53

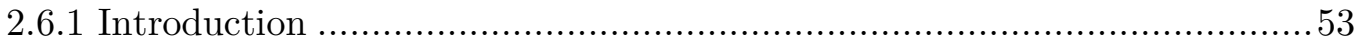

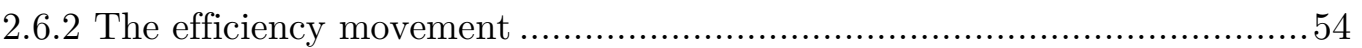

2.6.3 Approach to computer science …….................................................56

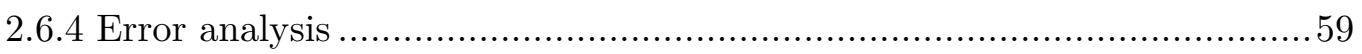

2.7 Planning under uncertainty ................................................. 59

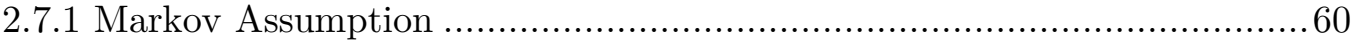

2.7.2 Markov Decision Process .................................................................... 60

2.7.3 Partially Observed Markov Decision Process .......................................61

2.7.4 Bellmans' Optimality Principle..................................................... 61

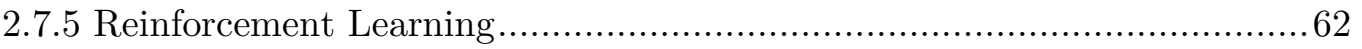

3 Error detection based on Task Modelling ............................................... 65

3.1 Cognitive Rehabilitation and supervisory algorithms .................... 66

3.2 Methodology for Task Modelling............................................. 68

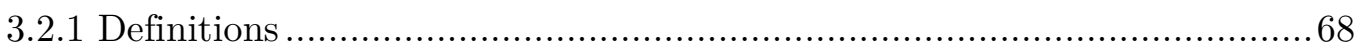

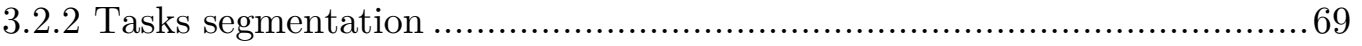

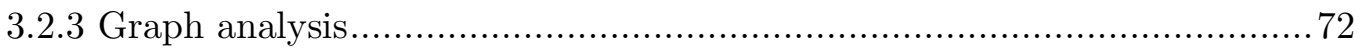

3.2.4 Classification of errors .................................................................. 74

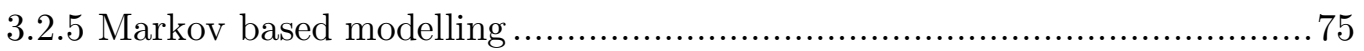

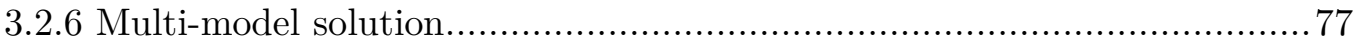

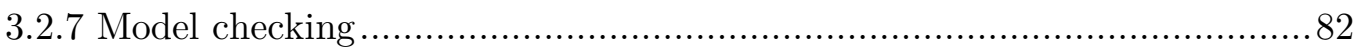

3.3 Functional description of the system ...................................... 83

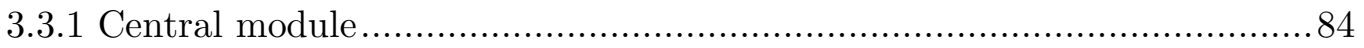




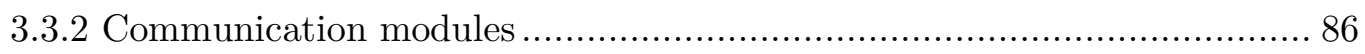

3.4 Developed tools for system management.................................... 90

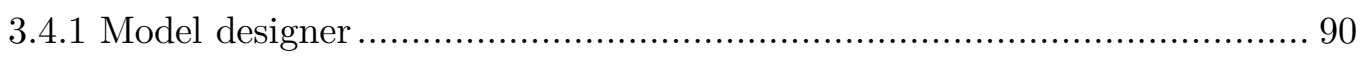

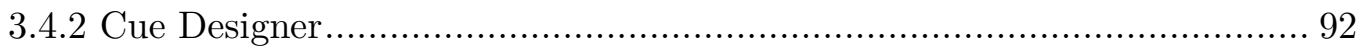

3.5 Lessons from Task Modelling ..................................................... 94

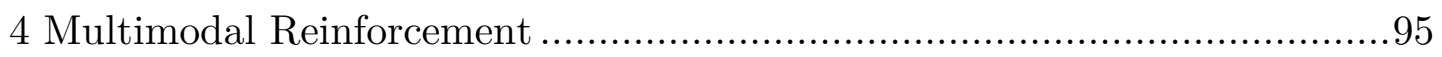

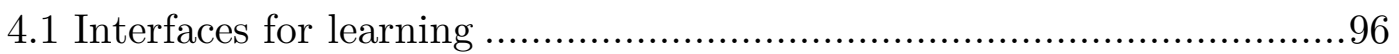

4.2 Feedback system description ............................................... 98

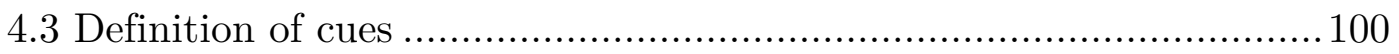

4.4 Cue design................................................................. 101

4.5 Considerations........................................................................ 102

4.5.1 Ipsilateral or contralateral............................................................. 102

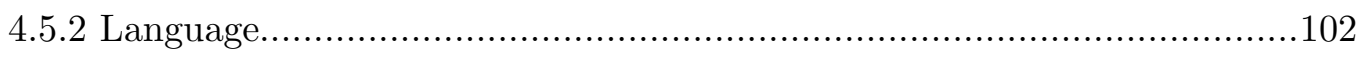

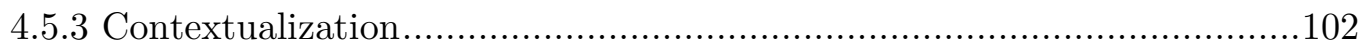

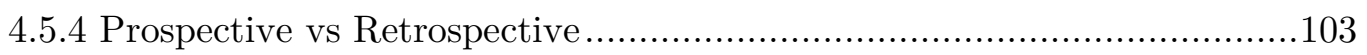

4.6 The multimodal interface .................................................... 104

4.7 Experiment for learning interfaces validation ............................ 105

4.7.1 Analysis of results from the experiment ............................................110

4.7.2 Conclusions from the experiment …..............................................112

4.8 Lessons about multimodal interfaces........................................... 113

5 Monitoring the user actions and performance ….............................. 115

5.1 Experiment for Natural User Interface sensor validation ................116

5.2 Experiments and Results .................................................... 118

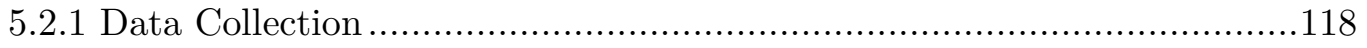

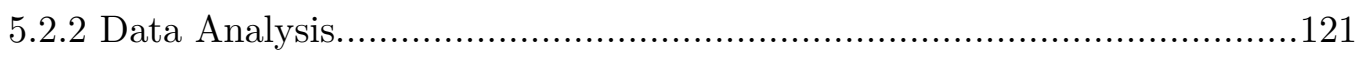

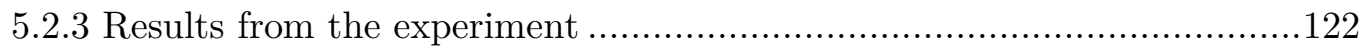

5.2.4 Lessons from monitoring user actions.............................................125

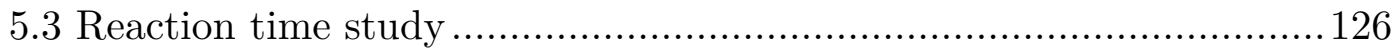




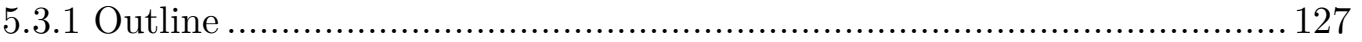

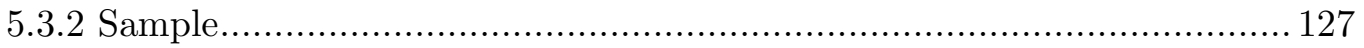

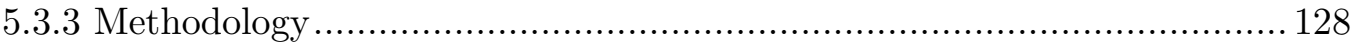

5.3.4 Lessons from Reaction Time study ................................................... 129

5.4 Monitoring physiological measurements .................................. 130

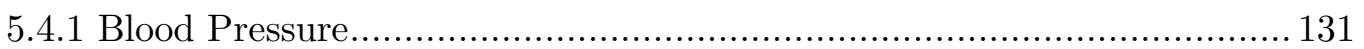

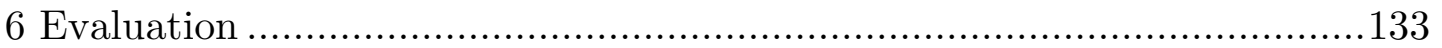

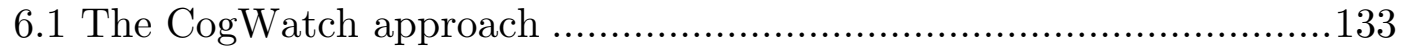

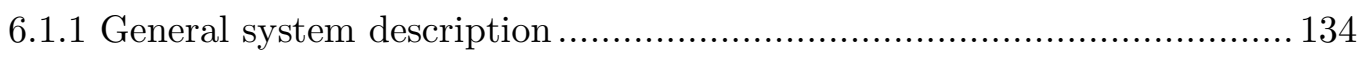

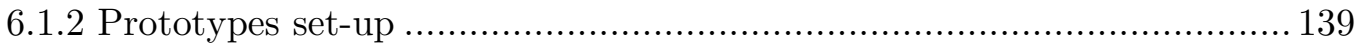

6.2 Technical evaluation .......................................................... 142

6.3 Health care evaluation................................................................ 143

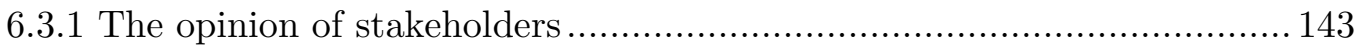

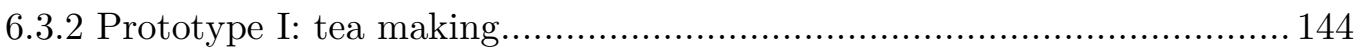

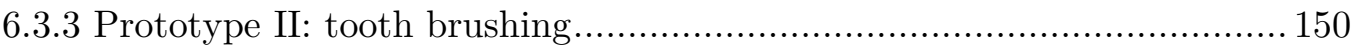

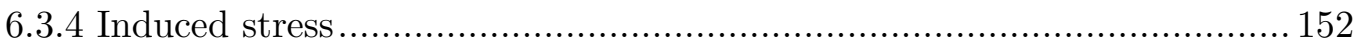

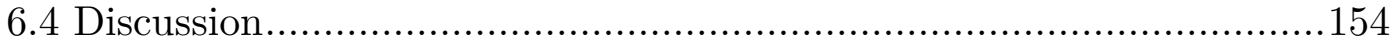

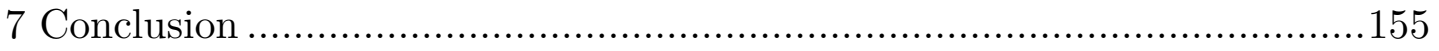

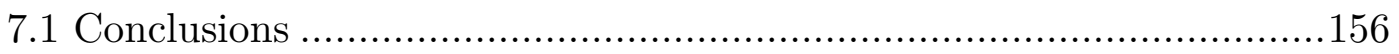

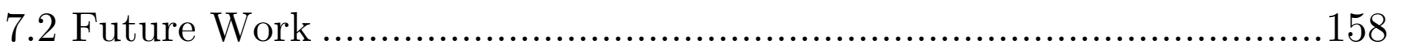

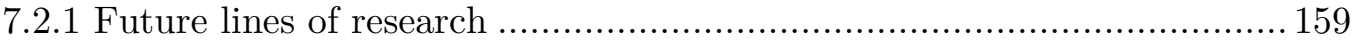

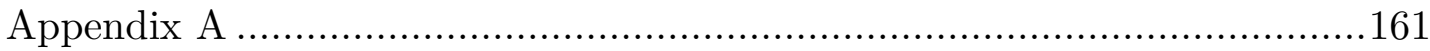

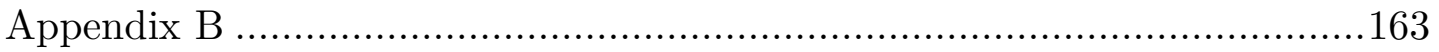

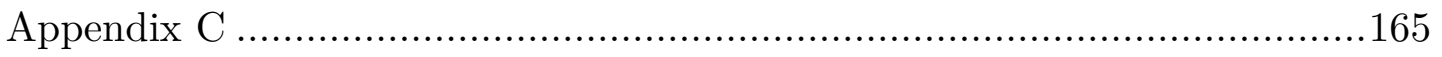

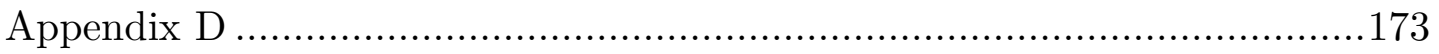

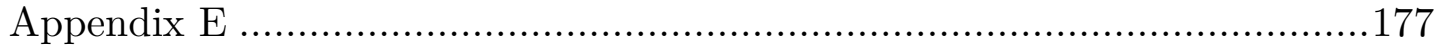

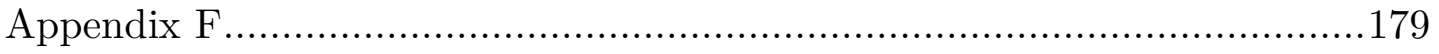




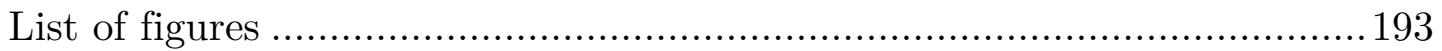

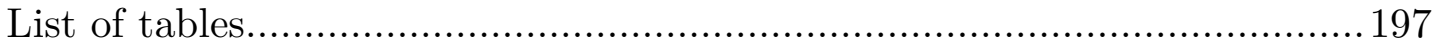

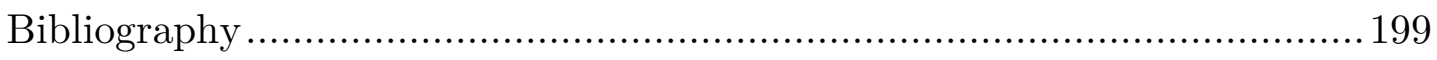





\section{Acronyms And AbBreviations}

AADS

ADL

AI

AmI

AR

BD

BORB

BP

BPMN

CEDI

COACH

CPG

CREAM

DDS

DA

D-L distance

FMEA

FreeRTOS

GA

HMM

HTA

IBM

IH

IMACS

INEF

IoT

IIR

MC

MCI

MCDM

MDP

MSE

MVC
Apraxia and Action Disorganisation Syndrome

Activities of Daily Living

Artificial Intelligence

Ambient Intelligence

Action Recognition

Big Data

Birmingham Objects Recognition Battery

Blood Pressure

Business Process Model and Notation

Centre for Studies on Inclusive Sport

Cognitive Orthosis for Assisting aCtivities in the Home

Consumer Packaged Goods

Cognitive Reliability and Error Analysis Method

Data Distribution Service

Decision Action

Damerau-Levenshtein distance

Failure Modes Effects Analysis

Free Real Time Operative System

Genetic Algorithm

Hidden Markov Models

Hierarchical task Analysis

International Business Machines

Information Handler

Interactive Manufacturability Analysis and Critiquing System

Instituto Nacional de Educación Física

Internet of Things

Infinite Impulse Response

MonteCarlo

Mild Cognitive Impairment

Multi-Criteria Decision Making

Markov Decision Process

Mean Square Error

Model-View-Controller 
NUI

OAET

OT

PARO

PGM

PI

POMDP

POSIX

PSL

PSMS

RFID

RL

RT/RTM

SHERPA

SOA

TAFEI

TD

TOTE

TUM

UML

UoB

UPM

VI

VTE

W3C

XML
Natural User Interface

Operator Action Event Trees

Occupational Therapy

Personal Robot

Probabilistic Graphical Model

Policy Iteration

Partially Observable Markov Decision Process

Portable Operating System Interface

Process Specification Language

Physical Self-Maintenance Scale

Radio Frequency Identification

Reinforcement Learning

Reaction Time/Reaction Time Meter

Systematic Human Error Reduction and Prediction Approach

Service Oriented Architecture

Task Analysis For Error Identification

Temporal Difference

Test Operate Test Exit

Technische Universität München

Unified Model Language

University of Birmingham

Universidad Politécnica de Madrid

Value Iteration

Virtual Task Execution

the World Wide Web Consortium

eXtensible Markup Language 
To my dearest... 



\section{CHAPTER 1}

\section{INTRODUCTION}

Information and Communication Technologies (ICTs) are taking an active role in our life. J. Ellul wrote in [1]:

\footnotetext{
"No technique is possible when men are free. ... Technique requires predictability and, no less, exactness of prediction. It is necessary, then, that technique prevail over the human being. For technique, this is a matter of life or death. Technique must reduce man to a technical animal, the king of the slaves of technique."
}

On the contrary of J. Ellul s' thought, and from a retrospective point of view, the work presented on this dissertation will defend the benefits from technology to educate and, therefore, the potential that technology have to preserve mental health. It is a bit pretentious, and even arrogant, begin a thesis contradicting one of the greatest philosopher of the last century, but, this is far away from the intentionality of the author. The actual pretension underneath the citation of this peculiar quote is to make the reader think about how the perception of technology has changed. Out of all chances, our brains have demonstrated an incredible capacity for adaptation to a new era where technology plays an important role in the way that millions of humans interact and communicate with each other. But, as J. Ellul foresight, relies on 
us how we use the technology and the huge amount of information that, nowadays, we can access.

This chapter introduces the work carried out on this thesis. After a brief introduction about the reasons and environmental conditions which have motivated the consecution of this work, the main objectives of this dissertation are established. At the end of the chapter, an outline of the thesis is described.

\subsection{Overview}

Task modelling plays a fundamental role in the way that humans take decisions and, therefore, make plans. The current work aims to present a study of the importance of modelling tasks. It has been studied how intelligent agents can adopt strategies based on modelling assignments and how to use this capability to help users to make decisions. The integration of a guidance system based on task models has been applied to the field of cognitive rehabilitation in order to evaluate their effectiveness. The conclusions of these thesis look forward open a discussion of how technology can be used to enhance tools for learning and reinforce our memories, both potential benefits for the technological society.

The work of this thesis has been founded by the European project COGWATCH (FP7-ICT-2011-288912) [2], which aimed to improve the way of living of stroke patients by creating a new personalized, long-term and autonomous way of providing cognitive rehabilitation.

Thanks to this project, and in collaboration with the University of Birmingham (UoB) and the Technische Universität München (TUM), we had the opportunity to contribute in the development of an innovative solution for patients who suffer from Apraxia and Action Disorganization Syndrome (AADS). By their side, we had learnt and come to the consecution of a successful breakthrough in assistive technology. 


\subsection{Research motivation}

Intelligent agents are defined weakly by M.J. Wooldridge and N.R. Jennings, as "a hardware or software-based computer system which has, at least, the following characteristics" [3]:

- Autonomy: "agents operates without the direct intervention of humans or others, and have some kind of control over their actions and internal state" [4];

- Reactivity: "agents perceive their environment (which may be the physical world, a user via a graphical user interface, a collection of other agents, the Internet, or perhaps all of these combined), and respond in a timely fashion to changes that occur in it" [3];

- Pro-activeness: "agents do not simply act in response to their environment; they are able to exhibit goal-directed behaviour by "taking the initiative"" [3].

- Social ability: "agents interact with other agents (and possibly humans) via some kind of agent-communication language" [5];

All of the above characteristics rely on a common property, the ability of making plans. The execution of multi-step complex tasks requires a major cognitive level and most regions of the brain are involve in the process [6]. Task modelling is inherent to human beings. It is one of the most important competitive advantages acquired by humans and a major manifestation is shown by the capacity of making plans. The complexity of this behaviour and variability, by the adoption of multiple strategies, comprises a fascinating challenge in the study of human skills. In the case of agents, multiple strategies have been followed to emulate task modelling [7]. But, these strategies, used to depend on an omniscient planner which supervise the tasks [8]. Usually, planning is associated with the term controller, when this happens; the term is used in charge of forcing behaviour [9, p. 2]. The problem arises when the environment does not met established conditions and solutions based on pre-known internal models of the environment fail. It is impossible to expect the unexpected, out of the working conditions. That is the reason why some scientist has turned their attention to new solutions inspired in the way that humans beings deal with them. Unless brain-inspired 
systems have achieved several milestones, this solution has not arrived yet $[10]$.

Is in this context where anthropological studies reveal the usefulness of the emerging technologies. Human-machine interaction is essential and ICTs' agents have come out as a natural step in the assistive technology for cognition. Visionaries such as J.C.R. Licklider predicted, back in the sixties, the role that computers could evolve in the future and the benefits that could bring the symbiosis between human and computers [11].

In the field of rehabilitation, new technologies have rolled a fundamental position during the last years. During this time different approximations of an intelligent and more independent home rehabilitation have emerged to meet the growing demand for assistance and caregivers in a family setting.

There is evidence of the increasing demand in the European home-care sector but some restrictions raise questions about the future supply of it. Economically affordable assistance and the availability of home-care workers are some of these challenges. One of the main doubts about home-care is whether sufficient qualified staff will be available if the ratio between the working age population and the elderly population changes [12]. ICTs applied to healthcare systems is proposed as a suitable solution and too many different technologies has been proved to properly coexist with the medical environment [13].

Medical practice and technology have grown together complementing each other. In the particular case of cognitive rehabilitation, some considerations have to be taken into account by the different aspects surrounding the patient. Therefore interdisciplinary collaboration is essential and begins with a proper definition of the needs of patients and the effectiveness of the methods used to achieve rehabilitation. According to this priority one of the aims of this thesis is to settle the bases to properly define those aspects, which results from the essential collaboration between different branches of the science. 


\subsection{Objectives}

The goal of this thesis is to establish some basis to define a platform useful for modelling and supervising tasks. Multiple approximations had been carried out to provide agents with this functionality. Current state of the art reflects some requirements for obtaining a suitable platform based on current technology. For this purpose it is necessary to define a framework for the applied technology to obtain the objectives of this thesis. This technology resides in the interaction between natural science, based on observation of natural phenomena, and design science, which attempts to create something. This dissertation has followed the framework proposed by S.T. March and G.F. Smith [14], see Figure 1.
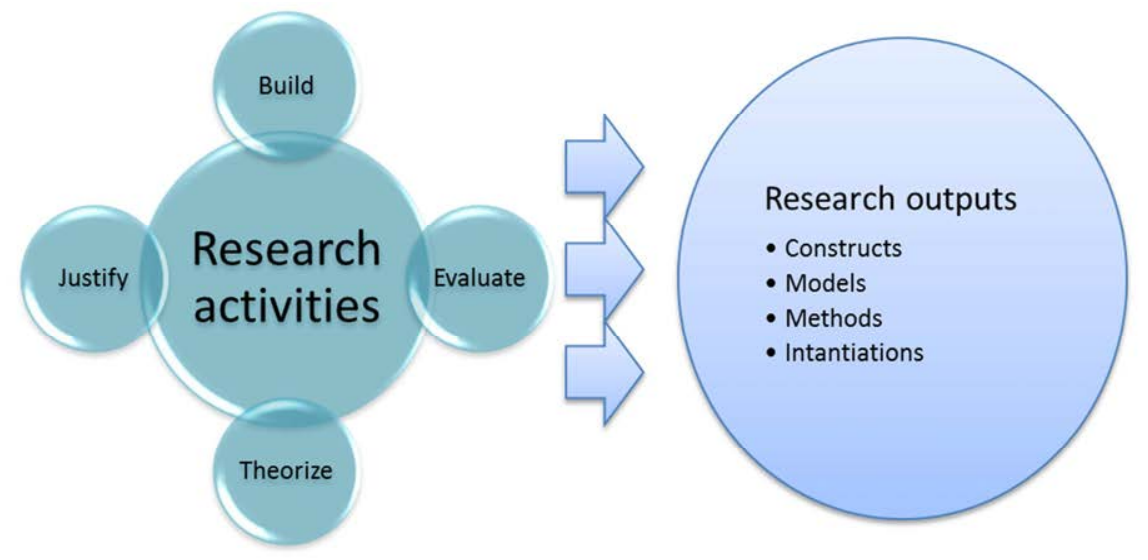

Figure 1. Research framework proposed by S.T. March and G.F. Smith [14]

Where the author distinguished two dimensions:

Research outputs:

- Constructs, constitute a conceptualization used for describing a problematic in a dominion.

- Model, an entity which establishes statements and propositions setting relationships among constructions.

- Method, a set of steps required for making a task.

- Instantiation, the realization of an artefact in their environment.

And research activities:

- Build, an artefact for making specific tasks.

- Evaluate, is an artefact for determining if a progress has been made. 
- Theorize, to explain the characteristics of an artefact and interactions with the environment.

- Justify, join evidences to demonstrate a theory.

The context of this thesis could be defined by the contributions in the form of models, methodologies, instantiations and constructs and comprises all the mentioned research activities.

This thesis is grounded on the following hypothesis:

- PGM will be best fit for establishing the relationship between subtasks in comparison to other methods. Specially, to determine those sequential errors more frequently done by users.

- Due to the multiplicity in the way of performing everyday tasks. It is an essential requirement the specialization of the system in order to obtain an adaptive system that meets the preferences of each user. Task Modelling could be an effective tool for gathering information from the expert.

- Finally, since it has been demonstrated the relevance of quantitative errors during tasks that involve the use of tools, the hypothesis that a rehabilitation platform that includes both types of errors, qualitative and quantitative, would improve the results obtained by the most common task model methodologies arises.

The following objectives are settled in order to contrast these hypotheses:

- Study and characterize the problematic and requirements for modelling the activities and errors made by users.

- Development of the software for monitoring performance of actions in multi-step tasks.

- Development of the software for assisting and provide feedback to users during the execution of multi-step tasks.

- Evaluation of suitability of task modelling for cognitive rehabilitation

- Assessment of the concurrent techniques for a Cognitive Rehabilitation System. 


\subsection{Outline}

The thesis explores different aspects of modelling tasks and their application to the field of cognitive rehabilitation. Thereby, different points where modelling tasks can bring improvements in systems to improve human factors are discussed.

The thesis is structured as follows:

Chapter 2: contains an overview of the most recent stage in the context of this disquisition, incorporating the newest ideas and features. The chapter introduces the problematic of stroke currently. Some of the cognitive disorders discussed in this thesis are described, including some inquiries about their aetiology, diagnosis procedures and manifestations. From this study, it can be established the basis for the definition of an effective rehabilitation platform. The principal attempts to carry out rehabilitation after suffering a stroke are described. After that, some of the traditional methodologies to gather information from the expert to construct specialized systems are detailed. The chapter continues with a description of the problematic of modelling. Finally, the chapter presents various models for analysing and modelling human sequence of actions. This study includes the topics addressed by Task Modelling, a brief historical review, and the description of current techniques for dealing with planning under uncertainty.

Chapter 3: entitled Error detection based on Task Modelling, presents the solution adopted for modelling ADLs. First, the methodology designed for Task Modelling is described in the following stages: task segmentation, graph analysis, classification of errors, Markov based modelling, multi-model and model checking. The chapter introduces the use of Markov Decision Processes (MDPs) which are applied to characterize the performance of tasks, specifically, in the case of multi-step task and their use for the detection of qualitative errors. A single evaluation of the performance of these algorithms has been carried out. From this evaluation it is determined the requirements of a complementary model for supervising the earlier stages via model checking and, therefore, include quantitative errors. The implementation of the system is later described for a deeper description of the work carried out. 
Finally, some additional tools developed to assist the professional in the development of models and feedback strategies are described.

Chapter 4: entitled Multimodal reinforcement is focused on the application of task model for guidance assistance and describes a cue system designed to cope with the work described in the previous chapter. After that the considerations to take into account for the assessment of a proper multimodal feedback are summarized. These considerations have been implemented in a multi modal interface for assisting the patient. Some experiments carried out to determine the suitability of multi modal interfaces are detailed. The chapter concludes with some guidelines for the implementation of multi modal interfaces.

Chapter 5: entitled Monitoring the user actions and performance, proposes the suitability of Natural User Interfaces (NUIs) and low cost tracking devices for monitoring patients' interaction. After justifying the use of this technology, in particular, the use of a depth sensor, other important aspects of monitoring the patient are studied. In relation with user performance, a study of the reaction time of users is presented. Finally the chapter reviews the monitoring of medical signals, in particular the relevance of blood pressure as a physiological signal for monitoring stress. This study is particularly important in the case of post-stroke patients.

Chapter 6: describes the test underwent to validate the system, exposes the results from these tests and takes particular attention to describe some clinical cases which shows the suitability of the system.

Chapter 7: summarises the main contributions of this work and conclusions about the work as a corpus. It proposes some interesting questions which have arisen from the thesis as well as potential future research which could be performed based on these. 


\section{CHAPTER 2}

\section{State OF THE ART ON TASK MODELLING AND POST-STROKE REHABILITATION}

This chapter contains an overview of the most recent stage in the context of this disquisition, incorporating the newest ideas and features. To introduce the problematic of cognitive rehabilitation the current state of the research in AADS is analysed, the principal attempts to carry out rehabilitation after stroke are described. From this study, it can be established the basis for the definition of an effective guidance platform. The requirements of this kind of systems have a common property: the importance of instilling professional knowledge in the system. To this end, it has been reviewed the more traditional methods in this regard. Finally, various models for analysing and modelling human sequence of actions are presented. This study includes the topics addressed by Task Modelling, a brief historical review, and the description of current techniques for dealing with planning under uncertainty.

\subsection{Cognitive disorders following a stroke}

A cerebrovascular disease happens when the blood supply to the brain is interrupted or there is a brain blood vessel broken. A stroke is produced due 
to the lack of oxygen or nutrients that the blood supplies to a specific region. Some of the symptoms could be the numbness or weakness felt in one side of the brain.

There are two main types of cerebrovascular accidents depending on the aetiology: caused by blockage, known as ischemia and caused by the leakage of blood in the brain tissue, known as haemorrhage. Ischemia is classified by the way that the blockage has been produced. A clot that forms a part of the body outside the brain can travel through blood vessels and trapped in a brain artery. This free clot is called an embolus and commonly is formed in the heart. When an ischemia is caused by an embolus is better known as embolic stroke. On the other hand, when the actors involved in the stroke are blood clots in an artery of the brain that remains fixed in the artery wall until it grows large, enough to block blood flow to the brain, In this case, the stroke is called thrombotic stroke [15].

The classical classification of cerebro-vascular accidents is shown in Table 1.

Table 1. General stroke classification adapted from [16].

\section{Ischemic stroke}

Transient ischemic attack

Reversible ischemic neurologic deficit

Stroke in evolution

Cerebral infarction: thrombosis, cerebral embolism, other entities (arteritis, hematologic disorders, ...)

Hypertensive encephalopathy

Lacunar infarct

\section{Haemorrhage stroke}

Intracerebral hypertensive haemorrhage

Saccular cerebral aneurysms

Arteriovenous malformation

Haemorrhagic disorders: aplastic anemia, leukemia, anticoagulants ingestion, thrombocytopenic purpura, etc

Thrombosis of the dural sinuses and cerebral veins. 
Depending on the severances of the stroke there could be multiple manifestations, some of these sequelae are listed below:

- Paralysis: weakness on a partial side of the body.

- Cognitive deficits: problems with thinking, awareness, attention, learning, judgment and memory. If severe the patient may have apraxia, agnosia or absence.

- Deficits in language: better known as aphasia.

- Emotional deficits: difficulty in controlling emotions or inappropriate emotional expressions.

- Pain: due to many factors, including damage to the sensory brain regions, inflexible joints or limb disabled.

Cognitive rehabilitation could bring to post stroke patients some benefits by reducing recovery times. A high percentage of the population that has suffered a cerebrovascular accident can undergo treatment to improve their living conditions. For example, the results reported in the study carried out by S. Rasquin et al. [17], in which 196 of the patients were tested neuropsychologically almost directly after their stroke, for the first time at 1 month, concluded that, while the frequency of post-stroke dementia is low (around ten percent), the frequency of post-stroke mild cognitive impairment (MCI) is high. The study concludes that in most of the cases the improvement of cognitive function is possible.

Hereinafter this dissertation will deal with the problematic of cognitive rehabilitation focussed on two particular syndromes: Apraxia and ADS. Even though, these syndromes are usually concurrent with many other possible manifestations.

\subsubsection{Apraxia}

The term Apraxia associated to cognitive disorders was firstly coined by Steinhal in 1871. After him the term was referred to a wide variety of neurologically induced, acquired and developmental disorders including buccofacial apraxia, constructional apraxia, dressing apraxia, gait apraxia, gaze apraxia, limb apraxia, speech apraxia, truncal apraxia and other 
disorders where the use of the word apraxia is associated with disturbance.[18].

It was H. K. Liepmann, see Figure 2, at the beginning of the $20^{\text {th }}$ century, the first who elaborate a description of apraxia as a distinct neuropsychological syndrome. His research and theories has a great influence on the consequent studies and diagnosis of the syndrome being responsible for their elucidation, distinguishing ideomotor, limb-kinetic or innervatory, and ideational apraxia [19].

H. K. Liepmann proposed the existence of the apraxic patients. According to him, the apraxia disorder is characterized because patients who suffer from apraxia have a correct concept of what they ought to do but cannot transform the image of the intended action into appropriate motor commands [20]. From the study of patients with right and left brain hemisphere damage, H. K. Liepmann come out to the conclusion that apraxia is associated with left brain damage $[5,6]$.

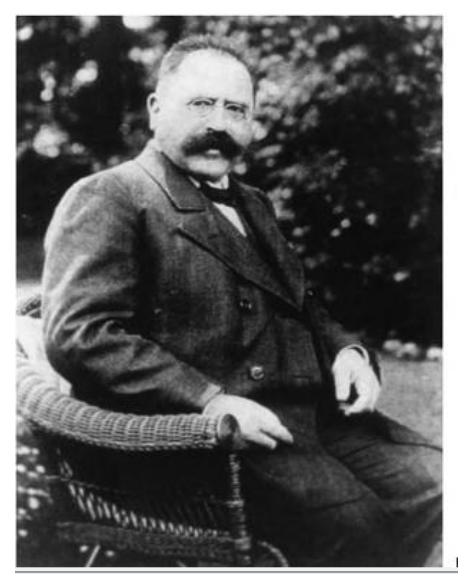

Figure 2. Hugo Karl Liepmann, extracted from [22].

After H. K. Liepmann, several definitions have been adopted to define apraxia which comprises one of the relevant topics on research in neuropsychology along the lasts two centuries.

A well accepted definition of apraxia is described in negative terms by L.J.G. Rothi and K.M. Heilman in [18]: 
"disorder of skilled movement not caused by weakness, akinesia, deafferentiation, abnormal tone or posture, movement disorders (such as tremors or chorea), intellectual deterioration, poor comprehension, or uncooperativeness"

The following taxonomy, showed in Table 2, described by K.M. Heilman et al. [23] summarized in the types of apraxia and their description.

Table 2. Different manifestations of apraxia from [23].

\begin{tabular}{|c|c|}
\hline ideational apraxia & $\begin{array}{l}\text { "an inability to correctly sequence a series of acts } \\
\text { leading to a goal" }\end{array}$ \\
\hline conceptual apraxia & "a loss of mechanical tool knowledge" \\
\hline ideomotor apraxia & $\begin{array}{l}\text { "a loss of the knowledge of how when making } \\
\text { transitive and intransitive movements to correctly } \\
\text { posture and move the forelimb in space" }\end{array}$ \\
\hline $\begin{array}{l}\text { dissociation } \\
\text { apraxia }\end{array}$ & $\begin{array}{c}\text { "a modality-specific deficit in eliciting learned } \\
\text { skilled acts" }\end{array}$ \\
\hline $\begin{array}{l}\text { limb-kinetic } \\
\text { apraxia }\end{array}$ & "a loss of hand-finger deftness" \\
\hline
\end{tabular}

For the scope of this thesis the description of the differential diagnosis of the thesis is not included, instead, a description of the main domains which constitute the core in clinical manifestation of apraxia is detailed in Figure 3. This omission is grounded to avoid divert attention to the study of problems in carrying out ADL experienced by patients with apraxia, more relevant to explain and link the contributions of this thesis ${ }^{1}$.

It is important to highlight that this clinical manifestations are caused by damage in different regions of the brain. Therefore, different studies have tried to isolate the location of these associated regions in order to characterize the disease.

\footnotetext{
${ }^{1}$ For a further review on this matter the reader could consult [18]
} 


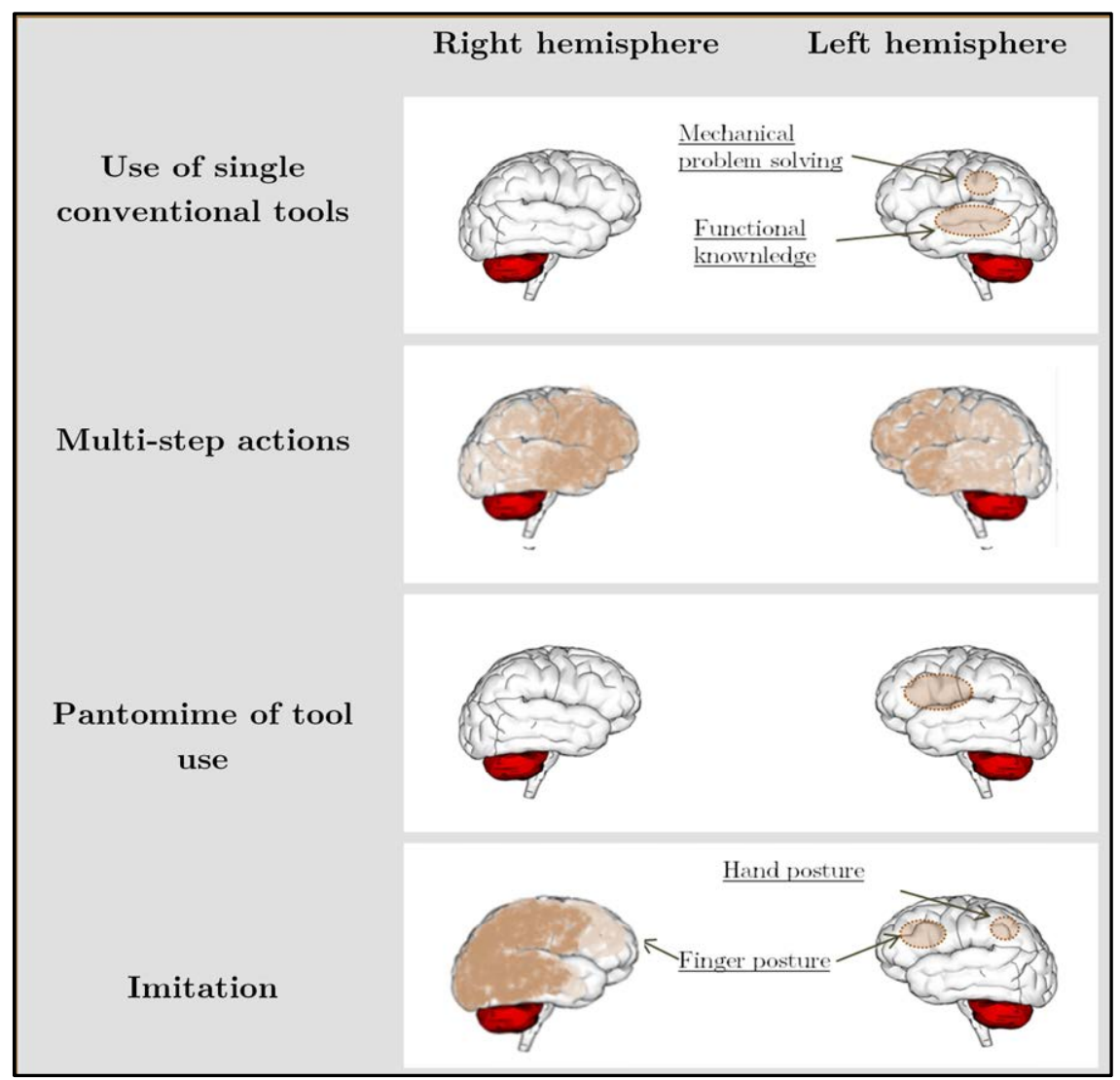

Figure 3. Summarized scheme of brain regions mainly affected by the apraxia adapted from[24]. Notice that the shadow areas refer to the potentially critical areas affected and it does not necessary mean that the whole area is affected, instead there are not founded grounds to delimitate the affected area.

\subsubsection{Action Disorganization Syndrome}

Another neuropsychological disorder following a brain injury is known as Action disorganisation syndrome (ADS). The term ADS has been used to describe patients with selective impairments in carrying out multi-step everyday tasks, like in the case of apraxia, ADS is not associated to motor deficits [25]. On the other hand, ADS is a neurological disorder which, unlike Apraxia, has been recently characterized. The first description of the symptomatology of ADS was described by Schwartz et al. through a case study. The authors highlight how disruption of performance may ensue when intentional control of action is compromised [26]. It could be associated with frontal lobe damage and leads to impulsive actions, poorly constrained by task goals. Patients may pick up the wrong objects or fail in the sequence while performing a task [27]. ADS is characterized by a high proportion of cognitive errors when performing everyday tasks [25]. 
There are very different manifestations of ADS, pattern of errors varies from one patient to another. V.g. G.W. Humphreys and E.M.E. Forde reported in [28] some case studies which represent this divergence among symptoms, as described by the cases of three patients (FK, HG and DS): in the case of patient FK, who manifested bilateral damage to the superior and middle frontal and temporal gyri, and lateral occipital gyri, due to an intoxication of carbon monoxide patient, the patient scored 24/25 and $25 / 25$ on views matching in the Birmingham Object Recognition Battery (BORB) [29]. However, this patient did have severe problems in the recognition of everyday objects and failed in the executive function. Another patient, HG, after suffering a stroke the scans revealed damage to the right inferior, superior, and middle frontal gyri, precentral gyrus, inferior parietal lobule and angular gyrus. HG was unable to name the colour of the ink in the Stroop test with colour words, e.g. "red, green, blue", was succeed in naming colours in non-colour words, e.g. responded black to the word "dog" but did not to the word "blue". Finally, patient DG, who revealed damage to the left inferior, superior, and middle frontal gyri failed in naming everyday objects. DG called a bowl a "jug or cup" a knife a "fork", and a lunchbox a "dolly". Earlier studies, such as [26] described a patient $(\mathrm{HH})$ with bilateral frontal brain injury. HH committed several errors while making a cup of instant coffee, some of the errors where putting butter into the coffee or adding coffee grinds into a bowl of oatmeal. During the performance of a grooming task, manifested perseveration errors, $\mathrm{HH}$ repeatedly turned the tap on and off, and wet the toothbrush several times. As previously mentioned, ADS is generally associated with frontal lesions, but it can also be manifested after a variety of lesions as reported in the case studies from [25], [28], [30], [31].

It is therefore permissible to think that, given the similarity of the manifestations of syndromes, apraxia and ADS. A platform for rehabilitation and assistance of patients of both syndromes is justified. Finally, in order to encompass both syndromes it is coined the acronym AADS, stands for Apraxia \& Action Disorganization Syndrome. 


\subsubsection{Performance of Activities of Daily Living}

The influence of AADS in ADL has been studied. The strategy followed in these studies is based on interviews and questioners with focus groups. These groups are integrated by relatives of the patient, Occupational therapy (OT) and other expert practitioners. The main drawback for the realization of these studies is given by the impossibility of realizing them in the patient's home environment. To overcome this drawback, researchers must move to rehabilitation centres for their studies.

The aim of these research is to understand how is affected a post-stroke patient in carrying out ADL. Some studies were focused on determining the task load implication, such as [25]. In [32], 33 patients of Apraxia were analysed to test ADL independence of stroke survivors. The study concludes that Apraxia treatment specifically improved praxis function and ADL. Another study based on observation studies the correlation between ADL motor and cognitive impairments and apraxia scores revealed from standard clinical tests in ADL task, such as washing the face and upper body and putting on a blouse or shirt [33].

The study of the Functional Independent Measure score as an outcome variable has been demonstrated as a correlated index to foresight the effects of the severity for performing ADL, established in [34]. Other similar scale is provided by the Physical Self-Maintenance Scale (PSMS)[35].

Finally, worth mentioning among studies of this nature a recent research noted for its application in a long data set establishing a low but consistence correlation in the scales given by the Barthel index, described in [36], and the Nottingham Extended ADL Scale [37], in a large group of stroke patients $(\mathrm{N}=635)$. The study concludes that their procedures offer a quick and valid way to detect apraxia and predict functional recovery for most stroke patients and define a rehabilitation plan [38].

\subsubsection{Error types and frequencies}

The methodologies described for error analysis based on HTA has been carried out in the case of action in simple, routine activities, along with a coding system for describing and analysing performance. From that analysis carried out by M.F. Schwartz et al. [39], [40] and L.J. Buxbaum et al. [41] from several case studies It can be concluded that there are six principal error types committed by AADS patients, see Table 3. 
Table 3. Summary of error taxonomy, from [42]. "Examples are drawn from the task of preparing a sandwich, snack and drink and packing the three items into a lunch box, as used with various patient groups by M.F. Schwartz et al. [39], [40] and L.J. Buxbaum et al. [41]"

\begin{tabular}{|ll|}
\hline Error & Explanation \\
\hline Step omission & $\begin{array}{l}\text { Failure to pack cookies, sandwich } \\
\text { or drink into the lunch box. }\end{array}$ \\
$\begin{array}{l}\text { Sequence: } \\
\text { anticipation/omission }\end{array}$ & $\begin{array}{l}\text { Packing the sandwich without first } \\
\text { wrapping it in foil. }\end{array}$ \\
Sequence: reversal & $\begin{array}{l}\text { Packing the cookies or sandwich } \\
\text { and then wrapping them. }\end{array}$ \\
Sequence: perseveration & Wrapping the cookies or sandwich \\
more than once. \\
Object substitution & Using apple sauce instead of \\
mustard on the sandwich. \\
Packing inappropriate items into \\
the lunch box
\end{tabular}

These errors can be classified into two categories omission and commission, according to R. Cooper [43]. Omission refers to the failure to execute critical actions or sequence of actions and errors of commission refers to the errors committed while performing an action in an incorrect or inappropriate way.

Several case studies with AADS patients have shown that some error types are more frequent than others. Some studies, [26], [44], [45], conclude that patients with high error rates commits more omission errors while patient with low error rates persist on errors of commission.

\subsection{Rehabilitation after stroke}

A recent study in Spain [46] accounts the rate of stroke incidents in people 18 years or older. The rate found was 186.96 cases per 100,000 inhabitants per year. This study responds to a necessity, because there was a lack of studies on the incidence of stroke in Spain with large denominators on the population. In addition, the study has concluded a significant increase in the incidence figures in the coming years due to the progressive aging of the 
population [46], [47]. This trend is observed in all western countries [48]. Unfortunately, in many countries, stroke is a lower priority than other diseases despite its public health impact[49].

There are two main causes of stroke depending on the aetiology. The most common are those that are originated by obstructions, known as ischemia and, secondly, accidents caused by leakage of blood into the brain tissue, known as bleeding. Ischemia is classified by how the blockade has occurred. A clot that forms in a region of the circulatory system outside the brain can travel through the blood vessels and block an artery in the brain. This clot is called an embolus and it is commonly originated from the heart. When ischemia is caused by a plunger it is known as embolic stroke or embolism. Furthermore, in the case of the occurrence of blood clots in an artery of the brain, which remain fixed in the wall of the artery, to grow large enough to block blood flow through this, the episode is called thrombotic stroke. The different nature of the causes results in a high incidence.

Data published by the World Stroke Organization indicate that a third of population who have suffered a stroke, after this event, experiment in some degree the loss of motor or cognitive abilities that prevent them from practicing daily activities [50].

One uncertainty always been in the air in ICT oriented to rehabilitation is whether or not a machine interface would be effective replacing OT. Obviously the capabilities of an OT cannot be fully replicated by a machine, but in the case of a specific rehabilitation it is plausible to wonder if effectiveness of the rehabilitation would be committed. From an extensive revision of multiple studies in the field of cognitive rehabilitation, the general conclusion from the research about multimodal intervention was that all the reviewed studies, classified as class II and III by the strength of their evidence, suggest no variation between using a computer interface instead of the traditional rehabilitation provided by an OT [51]. Furthermore, a later update of the study highlights that, in the particular case of Apraxia, a strategy for training is recommended because it is more effective in improving ADL function than the conventional OT [52]. To this point, most of the means for providing feedback to the patient had been studied and evaluated. Some studies focused on providing practical guidance of the best uses for 
different technologies in rehabilitation [53]. Even more, an adaptive architecture which provides a framework for evaluating how cognitive rehabilitation principles can inform and advance the development of more effective assistive prompting technologies that could be employed in smart environments has been reported as they are not always useful in terms of timing, content or patients' constrains [54].

On the other hand, ICT have not been only proved for being suitable for the rehabilitation. They are also useful for assessing diagnosis of different impairments and cognitive deficits. Based on speech recognition, an expert system could be useful for differing between Dysarthrias and Apraxia of Speech which are complex disorders of speech that are produced by different neurological disturbances [55]. Any help to enhance the diagnosis of cognitive disorders involve a need for proper rehabilitation of patients. Speech recognition has also been used to help in the diagnosis of different neuronal disorders like autism [56]. But recognition and signal processing of speech is not the only support from ICT to assess diagnosis, combining functional magnetic resonance imaging, trans-cranial stimulation and kinematic monitoring, H. Poizne et al. reported a method to model neural motor disorders [57].

Nowadays, some commercial cognitive rehabilitation systems offer cognitive rehabilitation based on different solutions. Assistive robotics is a new field of robotics which has evolved with the emergence of different prototypes, most of them, focused on the recovery of the motor skills [58]. On the other hand the use of cognitive robotics provided very positive solutions to therapies such as PARO robot pleasantly welcomed by psychologists to various therapies [59]. The problem is the effective cost, financial constrains make these systems unaffordable for every pocket, but, they evidence a promising future for home healthcare.

Fortunately, there are other alternatives for achieving cognitive rehabilitation at home at lower cost. The day to day technology has emerged as a great ally which has allowed the emergence of new platforms for rehabilitation. Mobile phones are chosen as one of the suitable platforms due to its ubiquity and the continuously improved accessibility [60]-[63]. The problem with most of these solutions are that, in some cases, the ubiquity of the system is compromised 
to provide alternative interaction or monitoring the patient and the barrier using new technologies for elderly who are not always familiarized with them.

To conferring a more immersive solution other platforms relies on virtual reality and the use of rehabilitation games [64]-[66]. The solution provided by this technology usually supposes high cost in dependence of the virtual platform but has obtained promising outcomes [67].

Other technology, like CogWatch, requires human-computer interface and interacts with the patient through multiple feedback and monitoring devices placed at patients' home. Similar solutions can be found for the task of hand washing with patients having moderate-to-severe dementia provided by $\mathrm{COACH}$ [68]. The pro from this solution is that it does not require makers, against, this framework was proposed to be generalizable to other ADLs but no further work has been published [54]. Another similar system prompts and messages to assist patients with successful completion of tasks, directions, remembering schedules and other functions providing feedback with a screen placed in a belt [69]. There are some evidence that the system could help to improve rehabilitation but it has not been proved for a specific neuronal disorder [70]. 


\subsection{Gathering information from the expert}

In order to be able to raise useful models for actual practice, it is necessary to transfer expert knowledge to the model. This is customary work in multidisciplinary groups. In this practice, many methodologies for gathering information have emerged. Some of the traditional methodologies are explained below:

\subsubsection{Focus Group}

R.A. Powell and H.M. Single defined a focus group as "A group of individuals selected and assembled by researchers to discuss and comment on, from personal experience, the topic that is the subject of the research."[71] Focus groups are discussions led by a moderator. Focus groups have been traditionally used during the design of products, services and experiences to get feedback from people. Some drawbacks are that some focus group study results may not be generalizable and participants can influence each other.

\subsubsection{Probes}

A cultural probe is a method of collecting information about people, their context and their culture. The aim of this method is to record events, behaviours and interactions in their context. This method involves the participants to record and collect the data by themselves. This is a useful method when the participants are hardly accessible due to different causes. It is a useful technique when the activities being studied take place over an extended period or at irregular intervals. The method has evolved because it was originally refused due to the addition of bias. As reported by K. Boehner et al.[72]:"The original probes were presented as subverting methods, but tend to be picked up as a recipe or reproducible method."

\subsubsection{Observation}

This method involves observing people in their natural activities and usual context such as work environment. With direct observation the researcher is present and indirect observation the activities may be recorded by means such as video or digital voice recording. Observation does not explain the cause of behaviour. Obtrusive observation may cause 
participants to alter their behaviour. Analysis can be time consuming. Observer bias can cause the researcher to look only where they think they will see useful information. Indirect observation uncovers activity that may have previously gone unnoticed.

\subsubsection{Surveys}

Surveys are a method of collecting information. Surveys collect data usually from a large number of participants. Surveys may take the form of a questionnaire or a face to face interview. Some of the guidelines to create a survey are: follow a standard procedure, try to avoid complex questions, avoid the use of jargon, avoid information bias, ask one piece of information at a time and adjust the style of the questions to the target audience.

\subsubsection{Affinity Diagrams}

Affinity diagrams are a tool for analysing large amounts of data and discovering relationships which allow a design direction to be established based on the affinities. This method may uncover important hidden relationships. Affinity diagrams are created through consensus of the design team on how the information should be grouped in logical ways. It can be used to categorise models, for example the work reported in [73]. However, it is a time consuming method.

\subsubsection{Method}

This is a brainstorming method which is focused on creating volumes of ideas. The process is to ask for a particular problem to a group of people and then they have to suggest 101 possible solutions. Because the focus is on volume some ideas will not be useful, by contrast, the group will collaborate and most participants will try to contribute in order to fulfil the task giving ideas that in other cases would be omitted. 


\subsection{The goal of modelling}

The following reductio aims at provide evidence of the complications which involve the definition of a simple action and its' proper modelling. Lets' imagine that somebody needs to model a simple ADL such as pouring coffee into a vacuum flask. From an epistemic point of view, it is possible to study a phenomenon by understanding the interactions of a corpus with the environment and how it is modified. To that end, in the following example, some sensors have been placed in order to register the interactions between the user and the objects and the changes in the physical properties sensed. Suppose that the coffee pot is equipped with some inertial sensors as well as the tap of the vacuum flask. Additionally, the body of the vacuum flask is equipped with a temperature sensor inside and a pressure sensor on its bottom. Finally, a sensor camera identifies the objects and hands interactions of a single person, (region in green in Figure 4).

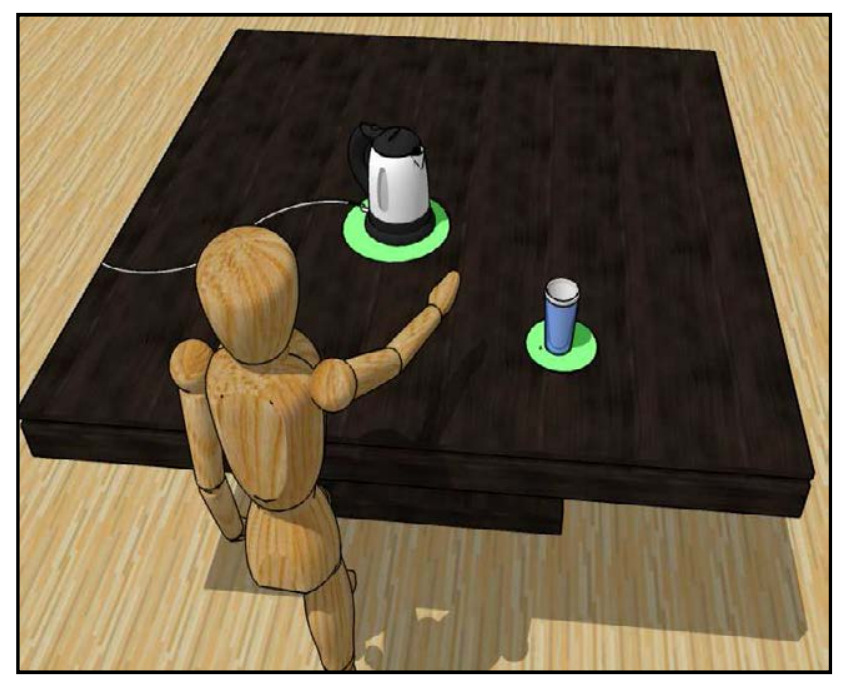

Figure 4. Introduction to the problematic of modelling; a simple task, pouring coffee into a vacuum flask.

From the measurements it has been identified different atomic events. These events can be recorded during the performance of an action. To simplify the problem lets' assume the following hypothesis:

- The events are processed sequentially at a high sample rate. That means that it doesn't matter if the subject triggers an event sequentially or concurrently because the system will always process it as a sequential buffer in the same order. 
- Sensors and communications channels are ideal (no occlusions, delays, etc).

- Events recognition accuracy is ideal (100\% of recognition rate without false positives)

The problem of modelling is to obtain a representation which can cope with all the possible combination of these events in order to identify the possible errors and succeeds of the subject during the performing of the task. The problem has been faced from different approximations: from recording the performances of different users and analysing the data to reducing the model to the simplest dimension isolating common errors and goals. In following sections it will be explained some of these approximations to deal with the problems of multiplicity of actions, efficiency and error recognition.

\subsection{Dimensionality of Task Modelling}

The aim of this section is to rough out Task Modelling giving examples of their use and letting a deeper description for the following sections where an introduction to historical approaches and applications are further described.

Task Modelling has a very wide field of application. It can be used to provide the content of instructions, to plan production processes or to define functional specifications. In modern applications it is quite common to see an architecture which relies on an internal Task Model for different purposes. In computer design, Task model describe how to perform activities to reach users' goals.

Task Model has been widely investigated for years without any agreement on a formal notation. They have been proposed various formalisms (such as formal grammars, transition networks, Petri nets) to meet different types of information for different types of Task Model. The discussion focuses on the fact that some are more geared towards identifying activities and logic decomposition while others are including indications of temporal relationships and adding information related to various concepts such as objects involved in a tasks, rules or agents [74]. In any case, Task Model could be considered a cornerstone for three main fields of application which are monitoring, contextualised guidance and decision making: 


\subsubsection{Monitoring}

The use of a task models has been applied under the framework of fault modelling. The aim of this procedure is to rely on quantitative and qualitative models of a physical system to contrast them with dynamic models, reducing diagnostic knowledge from qualitative simulations, continually comparing observations against fault-model predictions, and the gradual creation and testing hypotheses multiple failures. This process can be a valuable tool for revealing parameters of physical system over time by the time that a fault diagnosis is carried through [75].

Monitoring through a model can be applied to extensive areas of research. To cite a small sample of areas in which it has been implemented this kind of applications. Task modelling, for example, has been applied in manufacturability analysis with Interactive Manufacturability Analysis and Critiquing System (IMACS) methodology [7, Ch. 21], vehicles tracking [76], monitoring sensors [77], [78], or even in an area of major interest for the content of this work, such as, the description of ADL describing human, or other animals, actions at various levels of abstraction from raw poses and trajectories to motions, action models and activities. This models are interesting for classifying action sequences and study the relevance of each action by determining ordered or partially ordered trees of actions [79]-[81].

\subsubsection{Contextualised guidance}

A system can take advantage of an estimated current state through a Task Model to contextualize critical information in order to assist an agent. A significant example might be the use of these techniques aimed at creating an emergency evacuation plan for a building. A practical example of a case study is described in [7, Ch. 22]. Contextualization in AmI environments is also a key factor [82], as previously described, the use of activity-aware or policy-aware smart objects are really useful to achieve this objectives. In terms of usability and performance of an interface, contextualisation makes the difference. As an example, the main international standards organization for the World Wide Web, the World Wide Web Consortium, (W3C), has contribute in the best practices reference guides, publishing a 
review of different libraries and programs to take Task Modelling into account [83]. Furthermore the W3C highlights the role of Task Modelling in application design-cycle to improve performance and relax the definition of evaluation objectives through the definition of a model, see Figure 5.

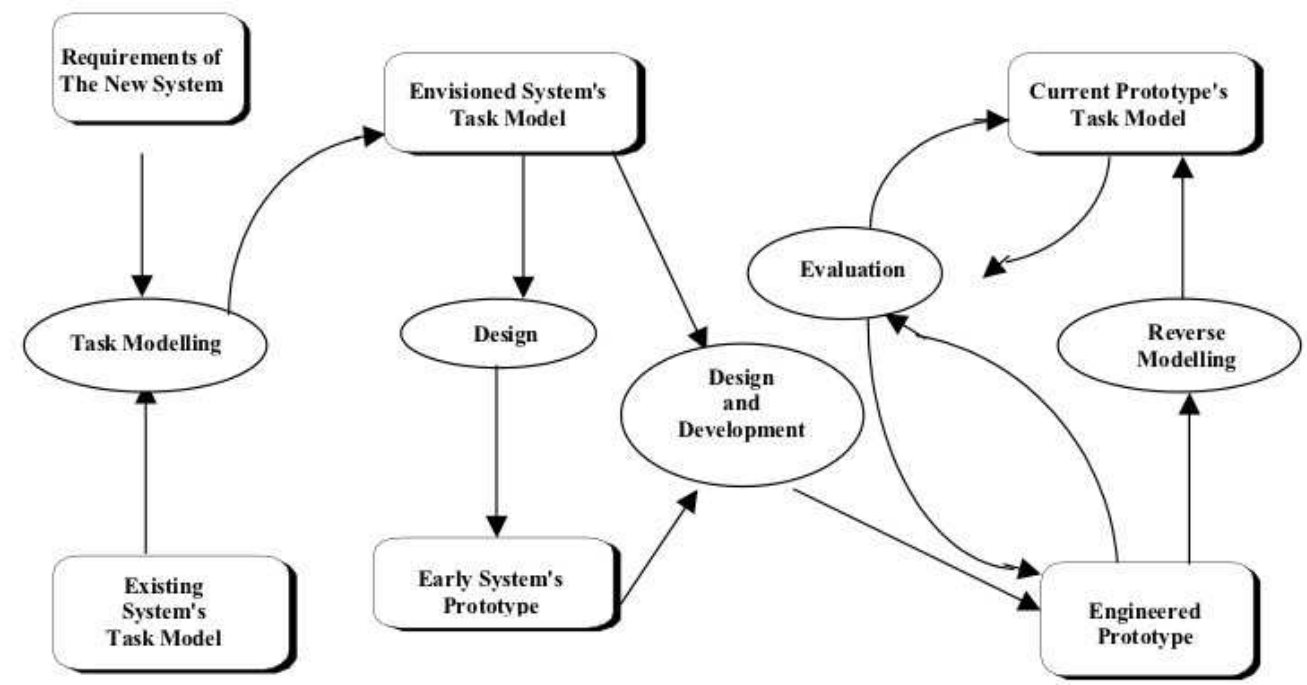

Figure 5. Use of task models in the design cycle from [84]

Regarding the use of a model in software development it is also worth to mention the use the Model-View-Controller (MVC) architectures nowadays, which is a widely extended architecture used in modern web applications promoted by companies such as Google, Inc. or Microsoft [85], [86], It divides a given software application into three interconnected parts, so as to separate internal representations of information from the ways that information is presented to or accepted from the user by contrasting it with a model via a controller, see Figure 6.

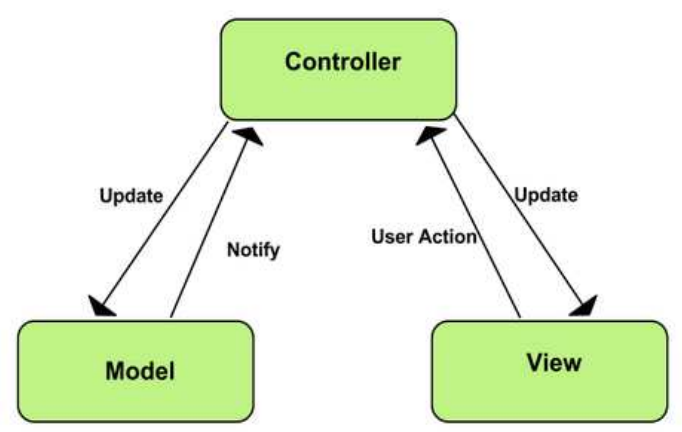

Figure 6. MVC architecture schema from [85]. 


\subsubsection{Decision Making}

Taking decisions is the principal application of Task Modelling and a vast field of study. There are some situations where the user has to deal with uncertainty and a Task Model could rely on multiple strategies for giving the best choice. These applications rely on the theory of decision postulated by E. L. Lehmann in [84] and [85] in some degree. Neo classical planning under uncertainty is based in MDP and model checking. The first focus the problem as a probabilistic map and deal with it as an optimization problem. The second deals with non-deterministic problems as a satisfiability problem focussing on reachability goals.

Theory of decision making has been clustered in the industrial applications under the Multi Criteria Decision Making field. This topic was firstly studied in operational problems such as production scheduling or inventory control [89]. The MCDM applications were later migrated to other decision systems such as energy planning [90]. The principal methodologies applied in this field are the DEA and Analytic Hierarchy Process (AHP). DEA is a nonparametric method of measuring the efficiency of a decision-making unit such as a firm or a public-sector agency. The problem of this methodology is that it relies on subjective definitions of the inputs and output and the models generated are not always generalizable.

AHP is the extension to MCDM of the approach followed in this thesis. It divides the problem into different sub-goals. The methodologies for reaching sub goals are modular and can be extended to more complex models.

Fuzzy sets are another strategy for simulating systems to support decision making. The problem is that it requires a vague definition of the system from the expert. Then, the modeller can construct a universe of discussion to set some abstract rules and simulate the system. The underneath true is that the modeller needs a deep knowledge of how the system works to properly tune the controller to achieve a good performance and the fuzzification process implies some level of abstraction. 
Genetic Algorithms is another approach to simulate systems. Studies their evolution over a period of time based on a natural selection process. The GA approach is hard to implement in real time systems because it relies on time consuming simulations.

From system identification branch it is possible to study a system presented in the form of a time series. The methodology relies on statistical tools to build models with deterministic and stochastic components. Previously, some suppositions about the ergodicity and the invariability in time of the system used to be made. There are a huge variety of models to describe a system depending of the internal correlations and the availability of the data, for example models described by L. Ljung in [91]. These models are applied to simulate and predict time series. It has been demonstrate useful in fields such as econometrics, energy demand estimation, seismology and many others.

Finally, another important approach to support decision making in the case where multiple rational agents are involved is the Game Theory. Game theory studies the conflict which arises from the interaction of multiple agents and the strategies to reach an equilibrium point. V. Neumann and Morgenstern are the ground-breaking authors who created this interdisciplinary research field applying the theory to the study of economic actors[92]. Later, J.F. Nash postulated his theory of equilibrium when he published the famous bargaining problem [93]. To name another important application, this theory was followed by J.M. Smith to the study of evolution [94]. 


\subsection{The study of actions}

The study of modelling tasks arose from the observation. It is undoubtedly a bio-inspired art. Back in the lately $19^{\text {th }}$ century, pioneers in the application of the modern technologies of the epoch or, simply, artist inspired by the beauty of capturing motion, initiate the study of modern biomechanics.

\subsubsection{Introduction}

It was 1985 when E.J. Marey published his study [95] about the registries taken by his own-made electromyogram. In the study, E.J. Marey analysed the pressures and reaction forces experimented by humans and other animals in motion, see Figure 7 . The study had a great impact among their peers.

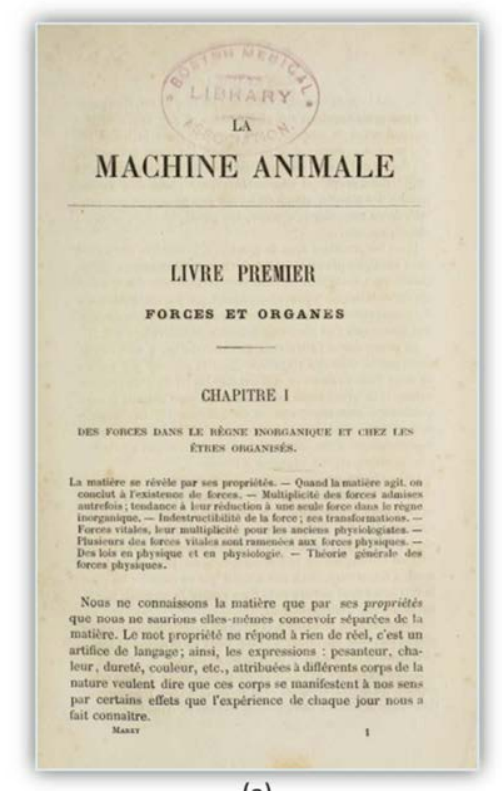

(a)

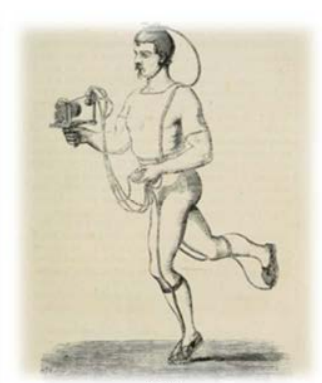

(b)

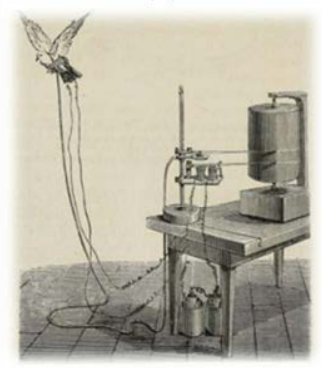

(c)

Figure 7. La Machine Animale (a) Cover, (b) sketch up of the study of human biomechanics and (c) sketch up of the study of birds in flight [95].

The influence of E.J. Marey was soon reflected in the work of other authors like E. Muybridge [96] or W. Braune and O. Fischer [97]. Their assumptions in novel analyses of gait in humans have withstood a century of progress and similar oscillatory movements are studied in the design of modern exoskeletons, such us in [98]-[100]. On the other hand, the technologies applied by E. Muybridge [101] capturing S. Gardner at a Gallop were revolutionary in the field of art. It has been seen as source of 
inspiration for Lumière brothers, who were finishing the cinematograph and, therefore, modern technologies for monitoring the performance of a task improved hereby.

\subsubsection{The efficiency movement}

The start of the twenty century and, in particular, industrialization, brought a twist of fate to the study of modelling tasks. Those responsible for this vagary were the leaders from the efficiency movement: F. Taylor, F. Gilbreth and L. Gilbreth.

The industrialization set up new criteria and methodologies for measuring productivity. Since an industrialist, H. Ford, sponsored the development of the assembly line technique of mass production, engineers started searching for ways of improving the value chain. In this context, F. Taylor developed his management ideas and postulated the four great underlying principles of scientific management [102].

The scientific management has been severally criticized by failing in the psychological approach and proposes models that specify the conditions under which individuals will become internally motivated to perform effectively on their jobs, v.g., the psychological model of job design described in [103].

On the contrary to Taylors' ideas and influenced by the background of Lillian as psychologist, the Gilbreths overdone their precise studies based on the figure of the worker [104]. They studied the net effect and the reduction of tasks applying an efficacy study of times and actions. To measure production times they decomposed all the tasks into some atomic units called Therbligs (the inverse of Gilbreth). The optimization of a task could be understood as the minimization of their associated Therbligs units. Note that these units refer both to cognitive and manual tasks. In Table 4 the most relevant Therblings units are showed. 
Table 4. Therbligs, along with their mnemonic symbols and standard colours for charting adapted from [105].

\begin{tabular}{|c|c|c|c|c|c|}
\hline Therblig & Color & Symbol/Icon & Therblig & Color & Symbol/Icon \\
\hline Search & Black & & Use & Purple & \\
\hline Find & Gray & & Disawemble & Violet, Light & \\
\hline Select & $\begin{array}{l}\text { Light } \\
\text { Gray }\end{array}$ & & Inspect & $\begin{array}{l}\text { Bumt } \\
\text { Orange }\end{array}$ & \\
\hline Grasp & Lake Red & & $\begin{array}{c}\text { Pre- } \\
\text { Position }\end{array}$ & Sky Blue & \\
\hline Hold & $\begin{array}{l}\text { Gold } \\
\text { Oclire }\end{array}$ & & $\begin{array}{c}\text { Release } \\
\text { Load }\end{array}$ & Carmine Red & \\
\hline $\begin{array}{l}\text { Transport } \\
\text { Loaded }\end{array}$ & Green & & $\begin{array}{c}\text { Unavoidabl } \\
\mathrm{e} \\
\text { Delay }\end{array}$ & Yellow Ochre & \\
\hline $\begin{array}{c}\text { Transport } \\
\text { Empty }\end{array}$ & $\begin{array}{l}\text { Olive } \\
\text { Green }\end{array}$ & & $\begin{array}{c}\text { Avoidable } \\
\text { Delay }\end{array}$ & $\begin{array}{l}\text { Lemon } \\
\text { Yellow }\end{array}$ & \\
\hline Position & Blue & & Plan & Brow7t & \\
\hline Akemble & $\begin{array}{l}\text { Violet, } \\
\text { Heavy }\end{array}$ & & $\begin{array}{c}\text { Rest for } \\
\text { overcoming } \\
\text { fatigue }\end{array}$ & Orange & \\
\hline
\end{tabular}

From their first study of bricklayers [106], reducing the task from 20 to 4 actions, to the familiar organization [107], by the application of their methodology, the Gilbreth marriage acquired a great reputation in the field of industrial management.

After the pioneers of the efficiency movement, the methods evolved, but the basis of tasks management were settled. 


\subsubsection{Approach to computer science}

We must move forward in the timeline until the mid-twentieth century, to situate the moment in which the computer science, from mathematical formalism, began to implement strategies to emulate behaviour in computer systems. This is the case of E. F. Moore and G. H. Mealy whose different interpretations of Karnaugh maps leaded to the definition of the sequential state machine [108], [109].

A Moore machine is defined as a 6 -tuple $(S, S o, \varepsilon, \delta, \theta, \gamma)$ where:

$S$ is a finite set of states, $S o$ is the initial state So, with $S o \in S, \varepsilon$ is a finite set of inputs, $\delta \varepsilon$ is a finite set of outputs, $\theta$ is a transition function such it maps a state and an input combination to the next sequential state $\theta: S \times \varepsilon \rightarrow S$ and finally $\gamma$ is an output function which maps each state to its correspondant output $\gamma: S \rightarrow \delta$

A Mealy machine is defined as a 6-tuple $(S, S o, \varepsilon, \delta, \theta, \gamma)$ where:

$S$ is a finite set of states, $S o$ is the initial state So, with $S o \in S, \varepsilon$ is a finite set of inputs, $\delta \varepsilon$ is a finite set of outputs, $\theta$ is a transition function such it maps a state and an input combination to the next sequential state $\theta: S \times \varepsilon \rightarrow S$ and finally $\gamma$ is an output function which maps each state and an input from the set $\varepsilon$ to its correspondant output $\gamma: S \times \varepsilon \rightarrow \delta$

Both extended solutions for synchronous circuits. The clear difference between Moore and Mealy machines are [110]:

- Mealy machine tend to have fewer states:

- Different outputs on arcs $\left(n^{2}\right)$ rather than states $(n)$.

- Moore machine are safer to use:

- Outputs change at clock edge (always one cycle later).

- In Mealy machines, input change can cause output change as soon as logic is done - a big problem when two machines are interconnected - asynchronous feedback may occur if one isn't careful.

- Mealy machine react faster to inputs:

- React in same cycle - don't need to wait for clock. 
○ In Moore machines, more logic may be necessary to decode state into outputs - more gate delays after clock edge.

E. F. Moore suggested that these sequential machines could be extended to probabilistic machines but his solution scopes only a finite set of deterministic states and inputs [108, p. 129]

Few years later C.A. Petri generalized the idea of distributed systems, clearly inspired by E.F. Moore and G.H. Mealy describing concurrent systems, into a mathematical representation known as the Petri net. This net represents a discrete event system in which it can be described the topology of a distributed system, concurrent or parallel. A Petri net is characterized by an initial state (named initial marking), two kinds of nodes, called places and transitions, and arcs, which represent connections of nodes. These connections must be done between nodes of a different kind, (i.e. place $\rightarrow$ transition or transition $\rightarrow$ place). Worth to mention that, unlike the models described below, it has a strong basis for analytics grounded in mathematics, an excellent review can be found in [111]. Furthermore, Petri nets include a graphical notation which makes use of symbolic notation, circles for places and bar/boxes for transitions, to make analysis more human-readable. This model is useful for determining reachability and deadlocks [112]. There are a several number of standardised models focussed, principally, on describing workflows and business processes. These models could be considered evolutions and applications of the ones described. To remark some of them: Unified Modelling Language (UML)[113], Process Specification Language (PSL)[112][114] and Business Process Model and Notation (BPMN)[115].

The use of a graphical notation was essential for the generalization of analytical models. This is the case of the models described henceforth. Another essential step towards modelling goals and plans as humans conceive them was the inclusion of hierarchies in the models. The work from G.A. Miller et al. was the first in considering this concept. The definition of three units of analysis where introduced for a goal oriented methodology called test-operate-test-exit, also known as TOTE [116]. The classical example of TOTE methodology applied to hammering a nail is showed in Figure 8. 
Notice that this model includes three important principles for modelling behaviour: information feedback, a control system and a hierarchical analysis of the system.

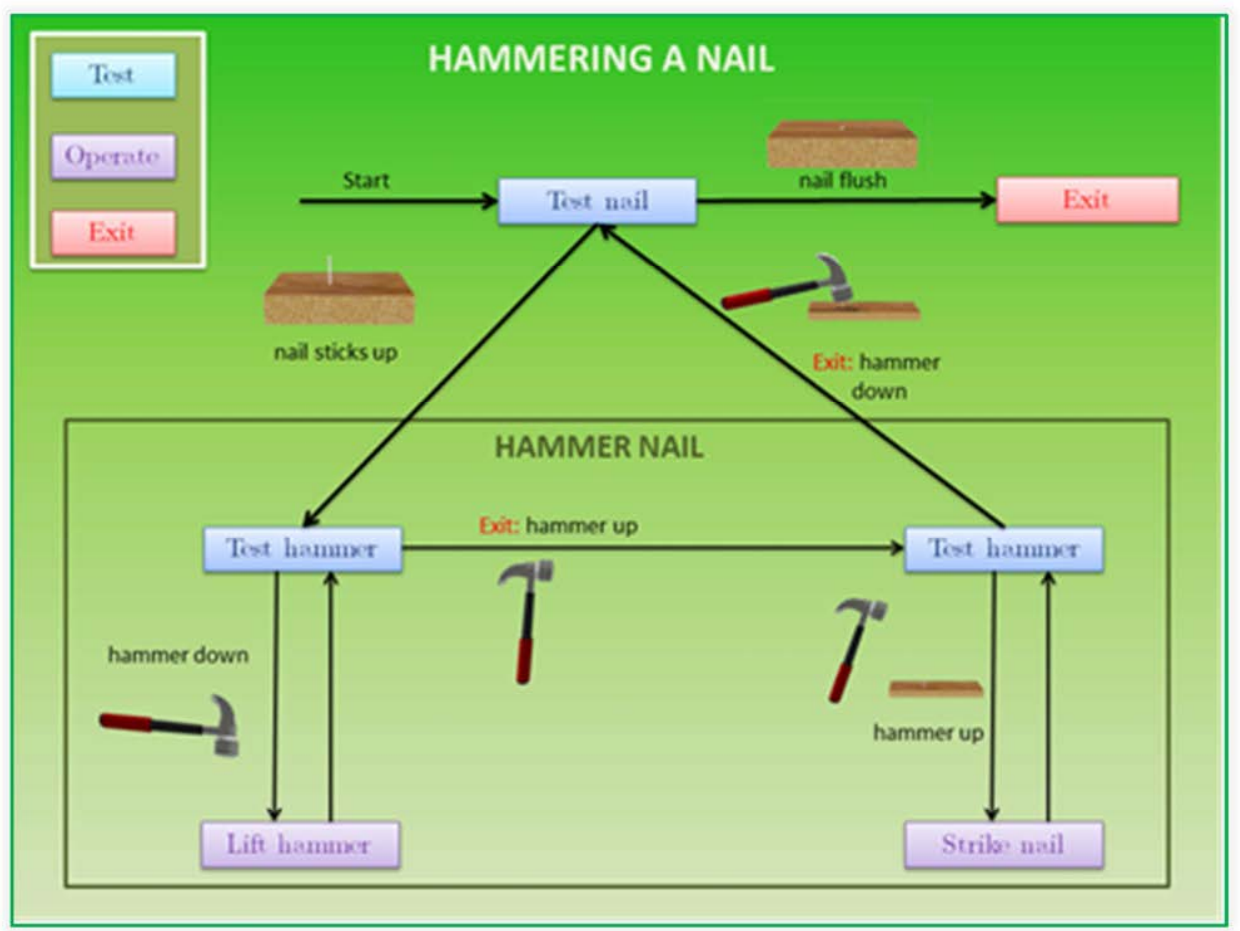

Figure 8. TOTE example of hammering a nail re-described from [117].

From this notion J. Annett and N.A. Stanton established the basis of a new task description method [118]. Some years later, J. Annett et al. developed the theory of Hierarchical Task Analysis (HTA). HTA is governed by the theory of performance which remains in three governing principles:

- To define a task at its' highest level it must be described by the objectives or goals of the task, called operations.

- Every operation can be decomposed into other sub-operations. These sub-operations are defined by sub-goals.

- The relationship between an operation and its' correspondent suboperations is hierarchical.

The use of HTA has a tradition of more than forty years in practice. The steps for carrying out this methodology where described in [119, Ch. 2], an updated re-description of the methodology can be found in [120]. 


\subsubsection{Error analysis}

HTA has been applied not only as a tool for definition of actions/operations in terms of goals, also for errors analysis. Human Error Identification has been consolidated as an inherited discipline of HTA. A classification of the most common human errors is shown in Table 5.

Table 5. Error types and associated Psychological Mechanisms from [121]

\begin{tabular}{|ll|}
\hline Error Type & Associated Psychological Mechanism \\
\hline Slip & Action execution \\
Lapse and mode errors & Memory \\
Mistake & Planning and intention of action \\
Mistake & Interpretation and situation assessment \\
\hline
\end{tabular}

Systematic Human Error Reduction and Prediction Approach (SHERPA) is intended to provide guidelines for human error reduction and quantification in a wide range of human-machine systems. The approach utilizes as its basic current cognitive models of human performance [122]. Another approximation described later was Task Analysis For Error Identification (TAFEI), it was defined by the need for theoretically-driven approaches that are able to provide practical utility in error prediction, an extensive validation of this method can be found in [123]. A comparison between SHERPA, TAFEI and other methodologies such as Cognitive Reliability Error Analysis Method (CREAM) in terms of sensitivity and performance can be found in [121, Ch. 8$]$

\subsection{Planning under uncertainty}

An agent, either human or computer, continually faces the problem of decision making under uncertainty. Based on limited information from the environment, the agent must take the best decision objectives agreed. On many occasions this process is repeated sequentially in time, so that in every 
moment the agent receives information and decides what action to take, based on their long-term goals. This is called sequential decision problems.

Classical examples of sequential decision problems are: an entrepreneur who must decide among several candidates who wants to hire based on getting the most benefit for the business, a broker who must decide, on the basis of limited information, to buy and sell shares to maximize their long-term utility or a robot which must navigate from one point to another, so that at each instant of time it must decide its' movements, based on information from its sensors and knowledge of the environment, to get in the most direct way to the target. The problems of sequential decision can be solved by, for example, genetic method which applied multiple simulations about the system, but when the system requires real time interaction the markovian framework is considered the best strategy.

\subsubsection{Markov Assumption}

A. Markov established that, in the case of a stochastic process it could be applied a memoryless assumption about the behaviour of the system. It implies that the conditional probability distribution of future states of the systems depends only on the present state; this is known as a Markov process.

\subsubsection{Markov Decision Process}

A MDP models a problem of sequential decision where a system evolves in time controlled by the actions of a user or agent. The system is determined by the transition probability function which maps the transition from one state to another as a consequence of taking an action $a .[124]$

It is formally defined as a 4 -tuple $(S, A, \emptyset, R)$ where:

- $S$ is a finite set of states, $S:\left\{\mathrm{s}_{1}, \ldots, \mathrm{s}_{\mathrm{n}}\right\} ; \mathrm{s}_{\mathrm{t}}$ is use for defining a state $\mathrm{s} \in S$ at time t.

- $A$ is a finite set of actions, $A:\left\{a_{1}, \ldots, a_{m}\right\} ; a_{t}$ is use for defining an action $a \in A$, made in a state $\mathrm{s} \in S$ at time $\mathrm{t}$.

- $\Phi\left(\mathrm{s}, \mathrm{a}, \mathrm{s}^{\prime}\right)$ is the state transition function. It is defined as the probability distribution, $P_{S S^{\prime}}^{a}$, of reaching a state $s^{\prime} \in S$. 
- $\mathrm{R}\left(\mathrm{s}, \mathrm{a}, \mathrm{s}^{\prime}\right)$ is the reward function which assigns a $\mathbb{R}$ value from reaching a state $s^{\prime} \in S$ doing an action $a \in A$ in a state $s \in S$.

\subsubsection{Partially Observed Markov Decision Process}

Not all systems are completely observable. Dealing with uncertainty means that there are situations where it cannot be known with confidence in which state the system is, given a time t. For that purpose it is necessary to include a measure of this uncertainty in the definition of the problem [125].

It is formally defined as a 5 -tuple $(S, A, \emptyset, R, O)$ where:

- Ditto as in MDP for S, A, $\Phi\left(\mathrm{s}, \mathrm{a}, \mathrm{s}^{\prime}\right)$ and $\mathrm{R}\left(\mathrm{s}, \mathrm{a}, \mathrm{s}^{\prime}\right)$

- $\mathrm{O}(\mathrm{s}, \mathrm{a}, \mathrm{o})$ is the observation. It is defined as the probability distribution, $P_{s a}^{o}$, of observing $o$ doing an action $a \in A$ in a state $s \in S$.

\subsubsection{Bellmans' Optimality Principle}

A policy $\Pi$ is a map for each state $s \in S$ and action $a \in A$ to the probability of performing that action $a \in A$ in a state $s^{\prime} \in S \pi: S \times a \rightarrow S$. The idea underneath determining the most desirable next state is to assign a cost function to indirectly define the desired performance. In order to determine a measurement, it must be defined the total reward expected from a policy $\Pi$ for being in a particular state, denoted $V^{\pi}(s)$, as well as the expected value of taking an action a under a policy $\Pi$, described by $Q^{\pi}(s, a)$,

$$
\begin{aligned}
& V^{\pi}(s)=E_{\pi}\left\{R_{t} \mid s_{t}=s\right\}=E_{\pi}\left\{\sum_{i=0}^{\infty} \gamma^{i} \cdot r_{i+t+1} \mid s_{t}=s\right\} \\
& Q^{\pi}(s, a)=E_{\pi}\left\{R_{t} \mid s_{t}=s, a_{t}=a\right\}=E_{\pi}\left\{\sum_{i=0}^{\infty} \gamma^{i} \cdot r_{i+t+1} \mid s_{t}=s, a_{t}=a\right\}
\end{aligned}
$$

Where $\gamma$ is the discount factor which takes values from 0 to 1 as a common infinite-horizon notation and $\mathrm{r}$ is the reward at a particular state.

By the use of these equations the problem is reduced to a maximization problem, (or in minimization problem in case of defining cost functions). Furthermore, the equations (1) and (2) previously defined are both 
Lyapunov nonlinear equations, this formulation is known as the Bellman equation and the optimal values can be defined as follows [126]:

$$
\begin{aligned}
& V^{*}(s)=\operatorname{argmax}_{a} \sum_{s^{\prime}, r} p\left(s^{\prime}, r \mid s, a\right)\left[r+\gamma \cdot V^{*}\left(s^{\prime}\right)\right] \\
& Q^{*}(s, a)=\sum_{s^{\prime}, r} p\left(s^{\prime}, r \mid s, a\right) \gamma \cdot \operatorname{argmax}_{a^{\prime}} Q^{*}\left(s^{\prime}, a^{\prime}\right)
\end{aligned}
$$

These are relevant functions for the evaluation of policies and values and determine the different approaches to solve it by the knowledge over the system, which depend on the transition probabilities and the reward function.

\subsubsection{Reinforcement Learning}

Reinforcement learning $(\mathrm{RL})$ is an area of machine learning inspired by behaviourist psychology and human physiology. It is also inspired, partially, by the theory of control. It aims to maximize performance. The underneath principle of reinforcement learning consists on a paradigm where an actor or agent perceives a reward or penalty signal after performing an action and tries to maximize the perceived reward in time. The problem of optimizing performance to adopt the best strategy has been described from the point of view if classical planning. RL takes the background from the markovian framework to present a solution to this problem. Some studies, such as [127], has proved the relation between reinforcement learning and the theory of control, in particular, the strategy of adaptive control.

R.S. Sutton and A.G. Barto are considered the "fathers" of RL They defined the basis of RL [128] and the architecture for the actor-critic structure, see Figure 9. There are different literature reviews which aim to summarize all the methods. Later reviews include new methodologies to deal with the problematic of scaling dimensions and large control spaces. The method described in chapter 3 is episodic, i.e. it does not require any kind of approximation, e.g. factorizations, eligibility traces, etc. This is justified because the approach described is based on finite sets of actions. For a better understanding of the RL theory the reader can refer to the following publications with a more detailed description of the field of RL 
and the available algorithms [126], [129]-[132]. The most important algorithms for RL in finite horizon scenarios are detailed below:

\section{Policy Iteration (PI)}

This algorithm fix an arbitrary initial policy, $\boldsymbol{\pi}_{\mathbf{0}}$. At iteration $\mathrm{k}>0$, compute the action-value function underlying $\boldsymbol{\pi}_{\boldsymbol{k}}$. Next given the value from $Q^{\pi k}$, it defines $\boldsymbol{\pi}_{\boldsymbol{k}+\boldsymbol{1}}$ using a policy greedy with respect to the previous value. After $\mathrm{k}$ iterations a better policy than the greedy policy is obtained. However, every computational step is higher than the value iteration method.[131]

\section{Value Iteration (VI)}

Using fixed point equations this algorithm develops forward in time methods for solving the optimal control problem [127]. Convergence in geometric time and the procedure of this algorithm is justified in [130]. It diverge from PI algorithm in the updating step, updating respect $V^{\pi k}$. This step reduces the problem to a recursion equation which converges independently of a stable initial policy (which is not the case of PI).

\section{Monte Carlo (MC)}

This solution allows episodic process to iterate solutions based on predictions via simulation. The goal of the algorithm is to estimate the optimal state-action value function defined as the expected cost of a session starting in state, taking an action and thereafter proceeding according to the optimal strategy until a final state is reached, a description of this process is reported in [133].

\section{Temporal Difference (TD)}

This solution describes a residual equation error in forward time. It is known as the TD error and considered combination of $\mathrm{MC}$ and dynamic programming. For a given policy the problem is to solve the TD equation for each time k. This can be solved with standard Gauss-Newton leastsquares method [134]. A famous application of this algorithm was reported by G. Tesauro in learning Backgammon game [135]. 


\section{Q-Learning}

This algorithm works by successively improving its evaluations of the quality of particular actions at particular states is able to compare the expected utility of the available actions without requiring a model of the environment. A description of the algorithm can be found in [136].

\section{Actor-critic structure}

The actor-critic structure is based on TD algorithms, as originally formulated the model is characterized by a separate memory structure to explicitly represent the policy independent of the value function.

An actor is represented by a policy, which selects the appropriate action. The evaluation of that policy is known as the critic. This critic is based on the TD error obtained at evaluation time. This scalar signal is the unique output of the critic and drives all learning in both actor and critic. This allows the optimization of the system by searching the optimal policy with gradient descent methods. This architecture has been extended to for more complex scenarios in continuous time, a review of different actor-critic schemas is reported in [137].

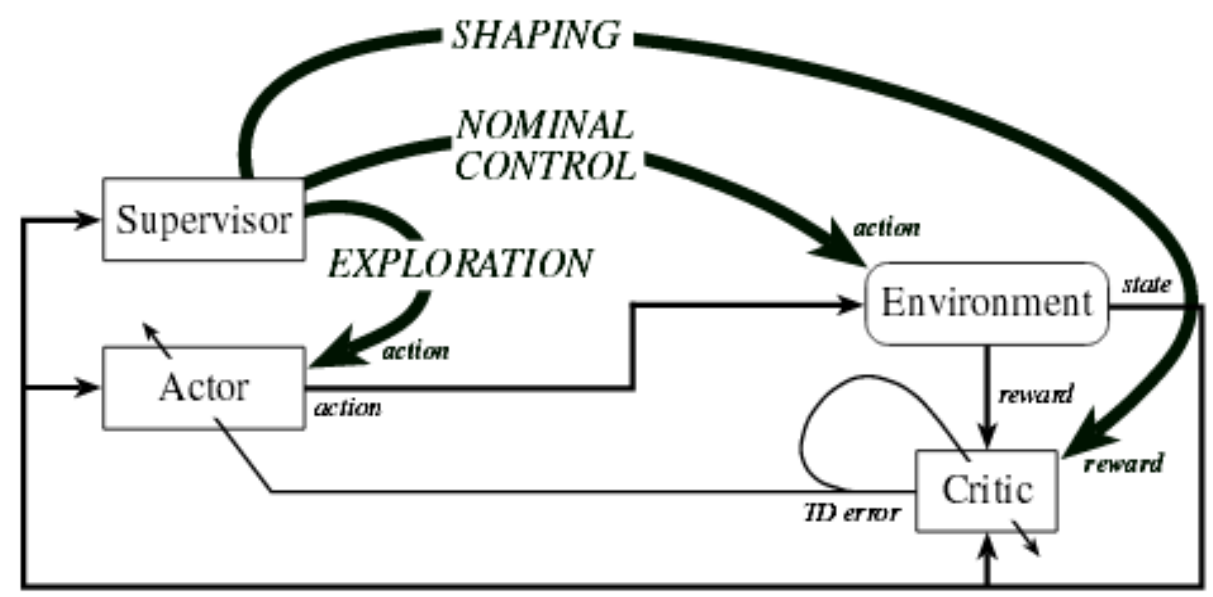

Figure 9. Actor-critic architecture and pathways for supervisor information from [138]. 


\section{CHAPTER 3}

\section{ERROR DETECTION BASED ON TASK MODELLING}

This chapter presents the solution adopted for modelling tasks. For this purpose, different stages have been developed. These stages are: i) segmentation of tasks within the framework of HTA, ii) the conception of a task model based on MDP and, finally, iii) a model checking monitoring system is proposed to adapt the system to the requirements of user. The chapter justifies the use of HTA and MDPs which are applied to characterize the performance of tasks, specifically, in the case of multi-step task, and their use for the detection of qualitative errors. A single evaluation of the performance of these algorithms has been undergone. From the results of the early evaluation of the system it has been found some constrains. Complementary to the foregoing steps, the last part of the chapter purposes a solution consisting of a supervising module defined by a structure named Cue Designer based on model checking. It includes quantitative errors and a solution to contextualise task modelling allowing the system to deal with the preferences of the user. The chapter concludes that the use of these strategies, MDP and model checking, could be complemented with the proper definition of the boundaries and interactions between both approximations. The result from a distribute strategy lead in a better experience for the user. 


\subsection{Cognitive Rehabilitation and supervisory algorithms}

Task analysis techniques have demonstrated to be very useful in order to define the problem and are widely used in order to identify and analyse human factor techniques. It can also be used retrospectively to identify and analyse the causality of a case study. In the case of cognitive rehabilitation it is important to identify which task analysis techniques are useful to use them as an input for human error analysis. The most notorious action oriented techniques are Decision Action (DA) flow diagrams, Operator Action Event Trees (OAET) and HTA according to D. Embrey et al.[139].

DA diagrams are charts which synthetize the sequence of action steps and questions to complete a task. This charts are similar to flow diagrams used in computer science and are based in simple binary decisions. Some withdraws from this method is that they do not describe the hierarchy of the task and goals, are complex to define and therefore are only affordable in simple tasks and don not offer guidance of which subtask must be described in more detail.

OAET are tree diagrams which represent a sequence of actions and decisions. The advantage from this methodology is that it allows the model of taking a decision or omitting that action which is useful to analyse the possible consequences of the operator decision. On the other hand, the method is not satisfactory for identifying decision errors not contemplated in the diagnosis. The method outperforms other when dealing with omission errors but fails in the approach to deal with commission errors

HTA, as described in the previous chapter, is by far the most implemented task analysis technique. But, there are some withdraws which difficult the implementation of this technique: the analyst needs to develop a measure of the skills of the user and the implementation of this methods requires collaborative work among the specialist and the analyst, this is a time consuming methodology in terms of specifications definition. Any tool for automatizing this process is considered a necessary good. 
Cognitive task analysis receives the "cognitive" property because it tries to identify the mental processes and decisions involved in the decision making process. Instead of focusing the attention on the task, cognitive processes are centred in the subject rewards and motivations for performing such task. The problem from this approximation is that it is grounded in the level of expertise. Expertise is considered a technical knowledge. It is acquired as a result of continuous and deliberate practice in solving problems in a domain[140]. The advantage from an expertise technic is that it lets the expert use their working memory in other task instead of focusing their attention in the domain in which they have specialised; even though they can perform complex tasks using a knowledge that they cannot describe. Some AI algorithms such as the famous Googles' AlphaGo algorithm are based on this idea of intuition from the point of view of an expertise gamer[141]. In a game where there are around $10^{107}$ possible configurations, professional players sometimes based their moves on what they feel right. The AlphaGo algorithm shares the intrinsic methodology from the procedures described in this chapter with an abstraction layer based on the use of Artificial Neural Networks. This approach is helpful to provide AI agents the capacity to learn complex activities without any kind of previous knowledge but, by contrast, the intrinsic abstraction makes difficult to transmit this knowledge to the user and therefore the final goal of rehabilitation cannot be easily achieved. Cognitive Task Meta-Analysis has been carried out to provide more evidence on the benefits that could result from trying to include in the model that information that the expert cannot easily transmit. This supposes an improvement in transmitted instructions [142].

The next step is the automatic analysis of the input from the analytic techniques. The reviewed literature focuses the problem mainly in two ways. On one hand the use of a decision tree is a possible solution. The use of decision trees allows the test of different models for automatically umbralise the values to determine the different diagnosis. One inconvenience from this method is shared with DA diagrams, decision trees performs better with dichotomous decisions. Another difficulty is the inclusion of the external factors that affect the judgement of the specialist at decision time. An application of this methodology has been reported by V. Venkatamaran et al. 
for a simple activity of "reaching to grasp"[143]. The study concludes the necessity of the definition of a global ontology for stroke patients to deal with the problem and provide further research.

On the other hand most of the rehabilitation systems decision support methodologies are based on markovian framework and suggested by different studies to cite some [54], [144], [145]. The COACH system found a successful solution for the task of hand watching [68] or the project Kinempt designed to assist individuals with cognitive impairments in preparing pizzas[146]. Despite reaching successful solutions both projects have failed in the implementation of their models on later systems; or there is no evidence of their existence. The hypothesis is that these systems are not readily reproducible or adaptable to other environments. In this chapter a process that does allow a simple implementation and has been proven in various tasks is defined. The methodology relies on the unification of three methods which compensate each other drawbacks, these methods are HTA, MDP and model checking.

\subsection{Methodology for Task Modelling}

This section describes the methodology proposed for task modelling, from the definition of the task until the obtaining of final models through internal definitions and the task errors. These steps are: task segmentation, graph analysis, additional errors, markovian based planning, multi-model definitions and model checking.

\subsubsection{Definitions}

In order to avoid confusions it is necessary to provide some definitions in the context of this section.

Task: a task is defined as a piece of work to be done. The task can be segmented in different sub-tasks. A group of subtasks defines a task and this definition is unique. If two tasks contains the same subtasks, then, are the same task, but, a task can be contained by another task with additional subtasks. 
Model: a model is a representation of a task. Different models can define a task. Two models can contain the same subtasks but not necessary be the same model, because models contemplate other properties of the task such as the order, repetitions, omissions, performance, time, etc.

Activities: the subtasks which define a task. Ergo, an activity is a task and then activities are iteratively defined. Hereinafter the term "activity" will refer to the sub-actions in which a task can be decomposed.

Activities can be classified into primary, secondary and error activities:

- Primary activity: a necessary activity to constitute the task.

- Secondary activity: an optional activity which does not influence the development of a task and cannot be considered an error.

- Error activity: an unnecessary activity to the constitution of a task, which influences the performance of the actor to achieve the target.

\subsubsection{Tasks segmentation}

From the study of the literature it has been seen different models to characterize processes and behaviour of humans performing a task. What is important in this approach is not simply the hierarchical decomposition, but also, the definition of 'plans' which complies with Human Factors. The use of HTA can be justified, in the scope of this study, by inherent functional properties from HTA, the definition of user goal directed actions which can be defined in goals and sub-goals allowing the use of recursion and the compatibility with techniques borrowed from Failure Modes Effects Analysis (FMEA) such as TAFEI[123] or SHERPA[122], mentioned in chapter 2 .

According to N.A. Stanton, the following steps must be carried out to the proper definition of a HTA [120], to illustrate these steps it has been chosen a simple operation to exemplify the process, see Figure 10:

- "Define the purpose of the analysis", in this case, change a bulb.

- "Define the boundaries of the system description", in this example case, it is desired to analyse the performance of a single operator with 
a given set of tools, see Table 6, supposing optimal conditions both for the installation facilities and the operator.

- "Try to access a variety of sources of information about the system to be analysed", in the example it has been used registers of users performing the task.

- "Describe the system goals and sub-goals", the different levels can be re-described, this description provides a non- deterministic view of the problem describing the system in terms of reachability.

- "Try to keep the number of immediate sub-goals under any superordinate goal to a small number", in the example from Figure 10 up to three sub-goals, depending of the level of detailed required for later stages small cardinalities could be critical to avoid explosion of data and allows to solve planning with exact methods.

- "Link goals to sub-goals", this step helps in aims of establishing an order of sub-goals, for example 1.0 followed by while waiting followed by 2.0 followed by when ladder ready followed by $3.0+4.0$ followed by 5.0 followed by if required 6.0 exit is a possible sequence among many others, the analysis of goals and sub-goals determine the dependencies between operations.

- "Stop re-describing the sub-goals when you judge the analysis is fitfor-purpose", this is the most important step and there are different formulas considering the level of detail required, in the example case it has been used a lower hierarchical level for describing "replace bulb" this is done in order to provide higher information in the case of a risky operation for the user. Task modelling is an iterative process and new models can be defined to describe sub tasks if more detail is required. This is possible due to the modularity nature of the system. 


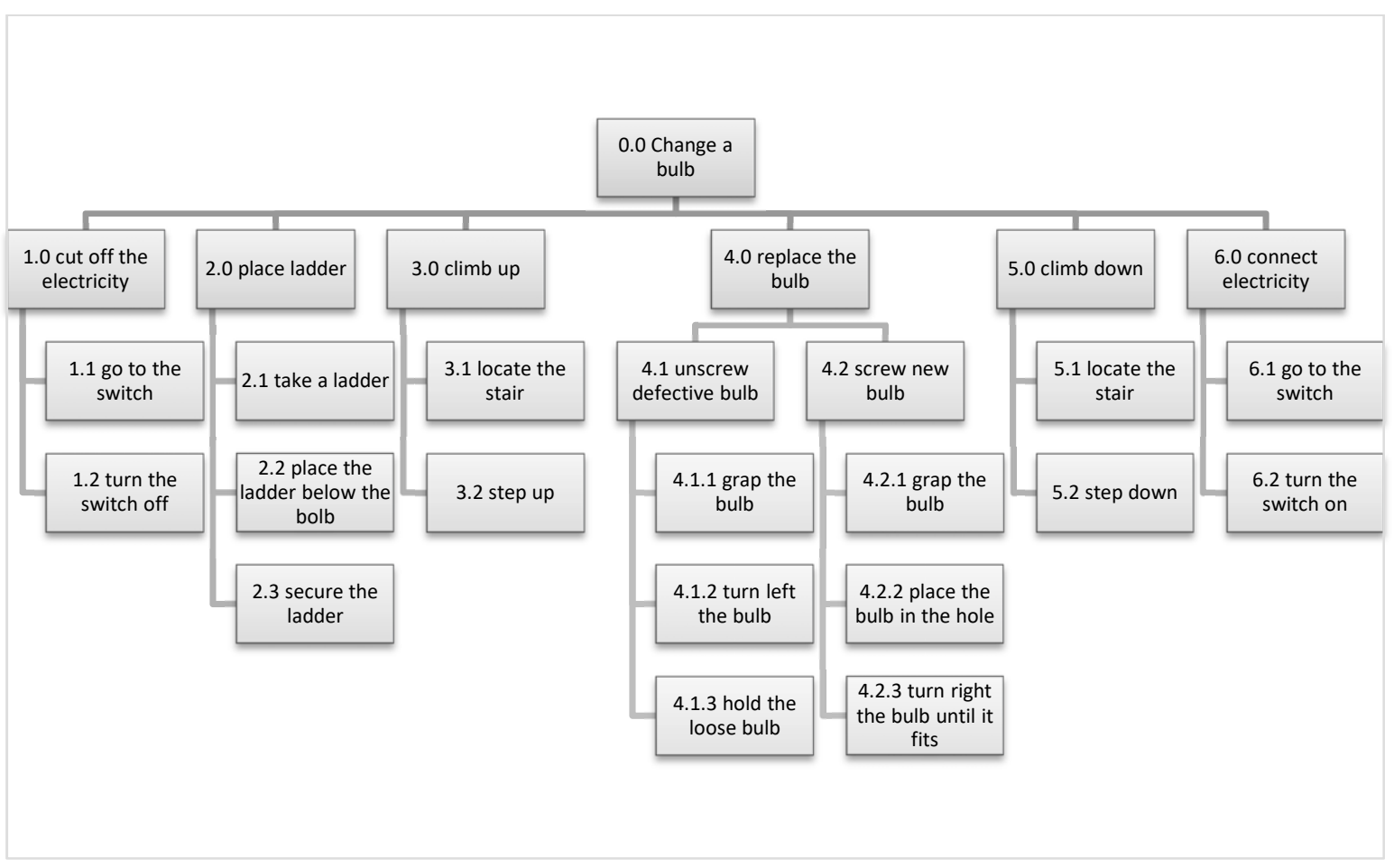

Figure 10. Example of a simple HTA for the task of changing a bulb.

Table 6 . List of required tools for changing a bulb.

\begin{tabular}{|cc|}
\hline Object label & Description \\
\hline $\mathrm{O} 1$ & Switch \\
$\mathrm{O} 2$ & Ladder \\
$\mathrm{O} 3$ & old bulb \\
$\mathrm{O} 4$ & new bulb \\
\hline
\end{tabular}

As previously mentioned, from the definition of hierarchies of the system it is possible to perform a process to determine errors in the execution of operations analysis. These steps must be performed under the supervision of qualified personnel. Cooperation between the computer scientist and professionals of the analysed task is essential, as it is critical to determine the relevant actions involved in the task related to the level of detail required and the observations from the user. Failure to define actions implies a lack of exponential order in the definition of errors. The definition of errors, however, could be corrected in later stages in the case that actions are well described.

Pre-classification of errors following the conclusions of the studies from L.J. Buxbaum et al. [41], M.F. Schwartz et al. [26] and R. Cooper and T. 
Shallice [42] are necessary in order to simplify later stages and characterize the performance of the average user.

\subsubsection{Graph analysis}

From previous analysis it can be characterized both actions and errors. The next step is transferring this information into a PGM.

By this action it will be obtained a second simplified description of the system and errors could be properly codified for computational purposes. It is also in this context that the fifth rule of HTA makes sense, since the low cardinality of the selected actions allows solving probabilistic graphs from exact methods and is not required to apply simplifications or abstractions with the consequent loss of information. It is essential information for the proper definition of assistance systems, as discussed in the next chapter. These is one of the benefits from this methodology, the system can be described in the same language used by the expert extremely simplifying the definition of requirements. On the other hand, the same lack of abstraction is required for the output of the system which will be directly perceived by the user. Information must not be lost, but also increased in order to enrich the communication of errors in accordance with the user preferences.

To give an example, from the HTA of Figure 10 we can define the following table of actions, Table 7.

Table 7. List of activities for changing a bulb.

\begin{tabular}{|cc|}
\hline Activity label & Description \\
\hline A1 & Cut the electricity \\
A2 & Place the ladder \\
A3 & Climb up \\
A4 & Replace the bulb \\
A5 & Climb down \\
A6 & Connect electricity \\
\hline
\end{tabular}

Given an initial state So, the following Probability tree could be defined as an explosion of all he possible combinations that is of an order: $\sim \mathrm{O}($ $\left.\sum_{i=0}^{N}(\operatorname{dim} A)^{i}\right)$ with $N \in[\operatorname{dim} A, \infty)$. Notation from HTA could be used to automatize this step, but, human supervision or an additional semantic 
description of the relationship among classes is required to accomplish this step and filling the missing information. The first solution is required in case of facing a new problematic and the second one could be appropriated in the case of a well described process where an ontology of all possible states is available.

The adoption of a PGM is useful in order to: i) review error definitions ii) reduce dimensionality of the tree by eliminating redundancy states and iii) detection of loops in the model. For the previous case of changing a bulb, see Figure 11, it can be clearly appreciated that, (linked to i)), S0003 is a terminal node and the system must prompt an alarm, once the system identifies that the user is operating under a risk, as a consequence, it leads to the identification of an omission error E001. On the other hand, (linked to ii)), all the nodes in red are considered impossible states, since transitions probabilities from their previous states are equal to zero, e.g. $P_{S 000-S 004}^{A 4}=0$. It re-describes constrains in the process, i.e., it is impossible for the user to climb down a scale without being on it. Finally, (linked to iii)), S029 is a virtual state; it could be seen as a bridge state because performing A5 in that state results in returning to S013. Notice that in this example it can be observed the implicit dimensionality reduction of strict sequential tasks in contrast with concurrent tasks. This effect, added to the benefits exposed of using a probability tree to define states, results in a dimensionality reduction. In the example case, the number of states is reduced from 55.956 theoretical states to 21 states, (orange states in Figure 11). 


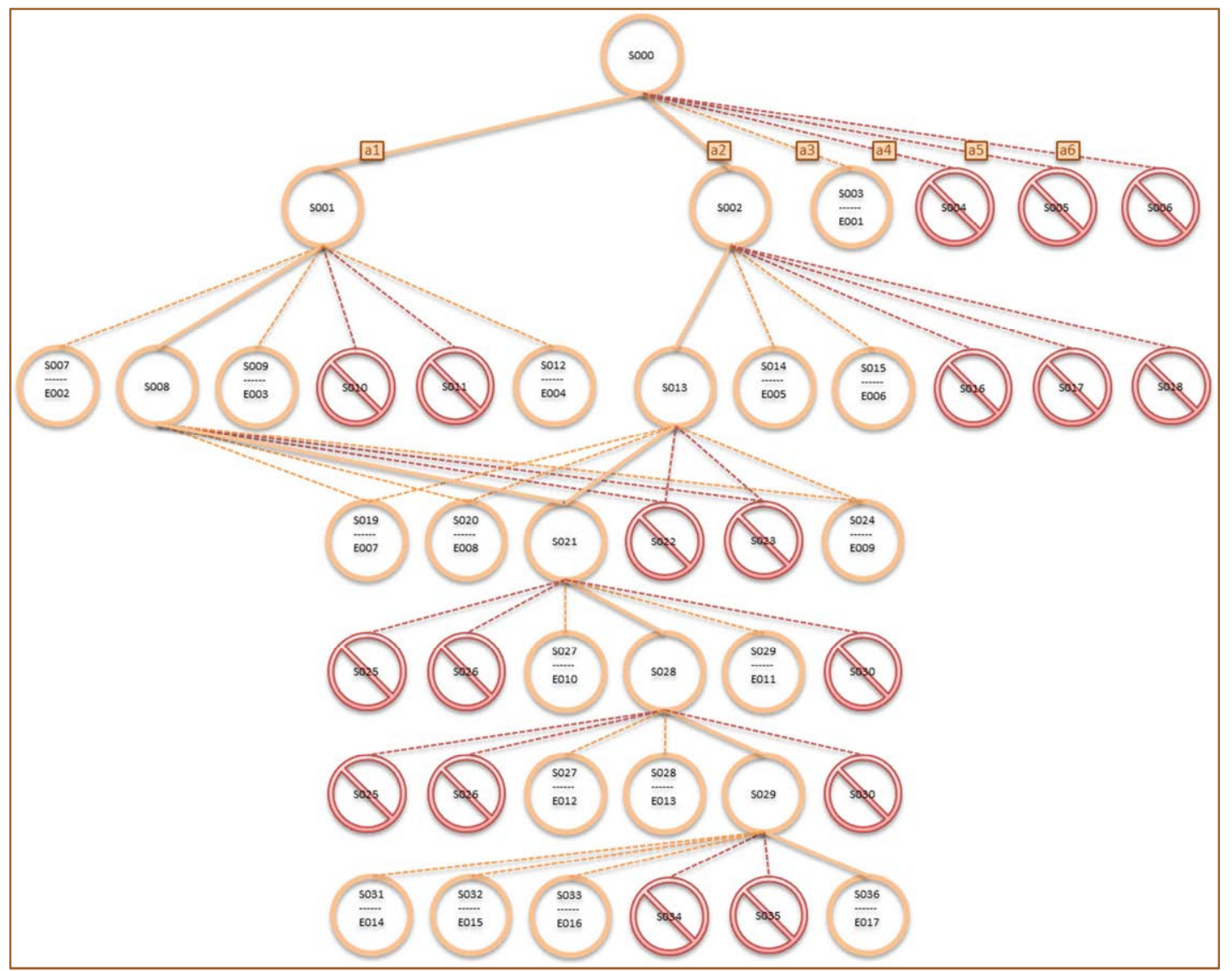

Figure 11. PGM defined from HTA shown in Figure 10.

After finalizing the error analysis from the graph exploitation of sub tasks, the next step is to consider the error activities previously defined. This activities leads directly to an error but were excluded from the graph analysis. At the end of this step it can be defined the final list of errors for the model.

\subsubsection{Classification of errors}

Each of the errors listed previously must be classified in order to define the action planning in the output stages, where severity of the error, priority and modality are specified. The errors can be classified as related to primary activities and secondary activities as: sequence, perseverations, omissions and toying. Additionally, semantic errors and additions are related to error activities. Quantity errors are only contemplated at this stage as a consecution of atomic actions but, as it will be explained later, it generates uncertainties on an appropriate definition. 
- Sequence error: perform an action in the wrong order.

- Perseverations error: (repeating an action or action sequence).

- Toying error: hesitation in movements and distractions.

- Addition error: add an extra component action.

- Semantic error: use of similar (semantic) objects instead of the correct one.

- Quantity error: use an inappropriate amount of ingredients.

It is also necessary to define the severity of the errors which has been classified in the context of this work on three different levels:

- Normal: the error can be corrected and does not interfere with the subsequent development of the task.

- Fatal: the error, due to its' severity, avoids the task to be continued.

- Safety: the error supposes a risk for the user and the system must try to avoid this possible risk with a warning.

\subsubsection{Markov based modelling}

While all the error activities could simply be mapped to an action plan, the errors committed by the user during the performance of the task depend on the current state in which the user is operating and in the possible transitions to other states. Omission, perplexity or user information requests also are context dependent. MDP constitute an ideal tool for dealing with this problem.

The graph can be supported with a MDP with the following characteristics:

- The action space A: Is a finite set of actions for each defined set. It can be added some common errors and potentially hazardous actions, excluded in the PGM, the reason is that any strategy of variable extraction can deal with these activities and they do not contribute to the goal of the process.

- The state space S: Is a finite set of states resulting from the dimensionality reduction. This finite dimension guarantees an exact solution by methods from reinforcement learning and dynamic 
programming by the use of recursive functions to take advantage of the programming pile.

- Transition function: this function states that the cumulative sum of all transitions possibilities from and state must be unitary and models the transition probability from one state to another by performing an action. A common notation this function is to deal with it as a kripke structure if transitions are modelled as a binary tree.

- Reward function: is used to determine the human factor or the opinion of the expert by adding a subjective value to reach a state and perform an action.

Once set the values of rewards the system can be solved by exact methods. The following algorithm has been designed as a possible method to solve the problem by taking advantage from the recursive stack by value iteration. If the inputs from the system are not clear it is possible to make use of different algorithms such as the POMDP algorithm described in Appendix B. 


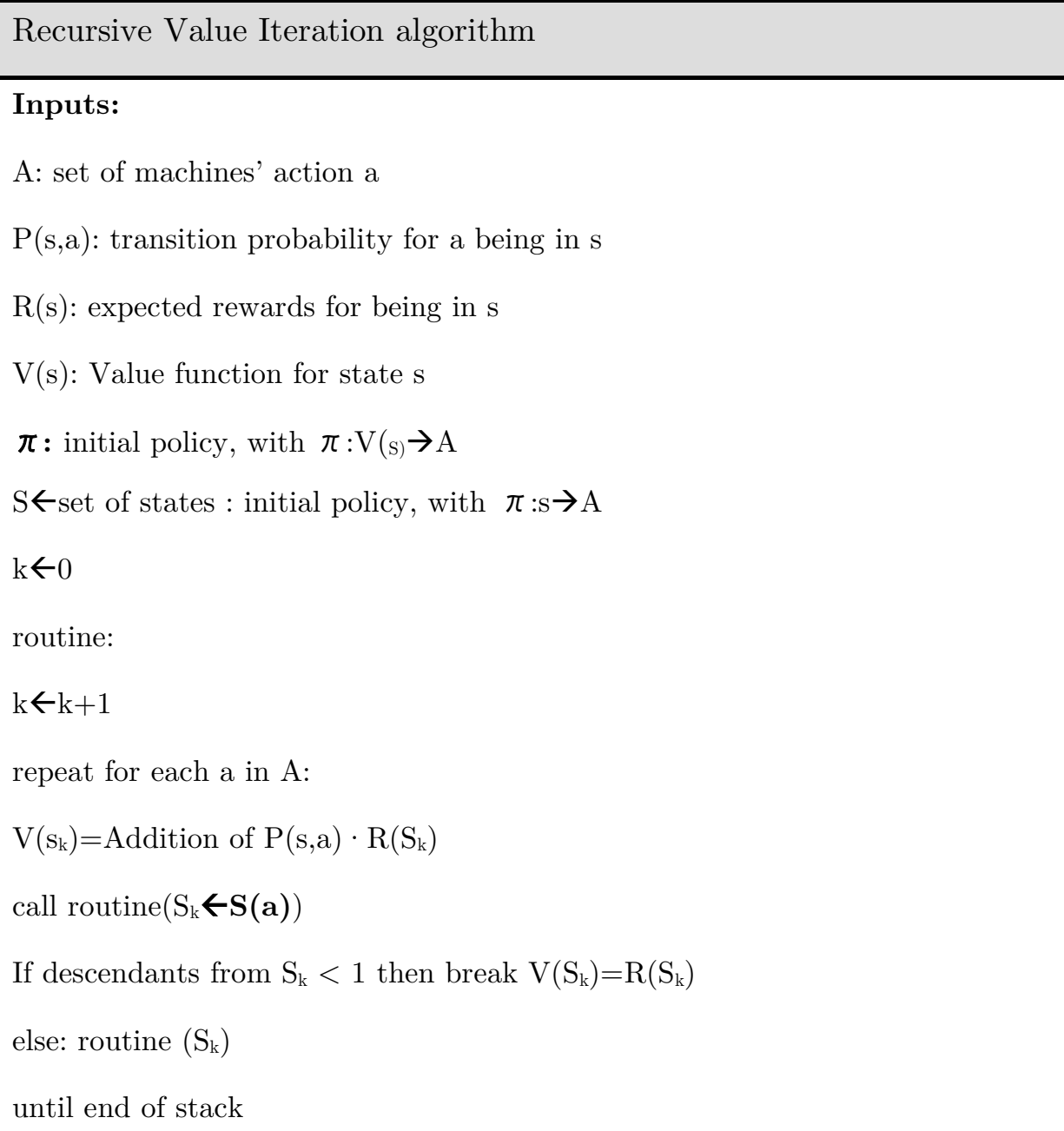

After solving the MDP is possible to adopt an actor critic structure to supervise the evolutions of the task and evaluate user actions. At the end of this chapter a descriptive architecture of the system is provided to find a functional description on this matter, see section 3.3.

\subsubsection{Multi-model solution}

Also, it is difficult and tedious to model all the possible preferences of the user in terms of discrete units.

To exemplify this problematic the results from the first evaluation of a complete assistive prototype based on MDP are presented.

The MDP task model is described in [189] and follows these assumptions:

- The action space A: Is a finite set of actions given by the HTA carried out for tea preparation described in [190] 
- The state space S: Is a finite set of states resulting from the dimensionality reduction.

- Transition function: since the activity must be supervised by an expert the transition function follows the kripke modality assuming binary transition probabilities.

- Cost function: incorporates human judge to the actions of the user, in this sense the faster strategy for carrying out an activity is not considered always the most psychological plausible and the strategy to proceed is conditioned by this function.

The cues where provide in a hierarchical static order according to the floe diagram showed in Figure 12. This was the first approach made to provide a fixed feedback structure.

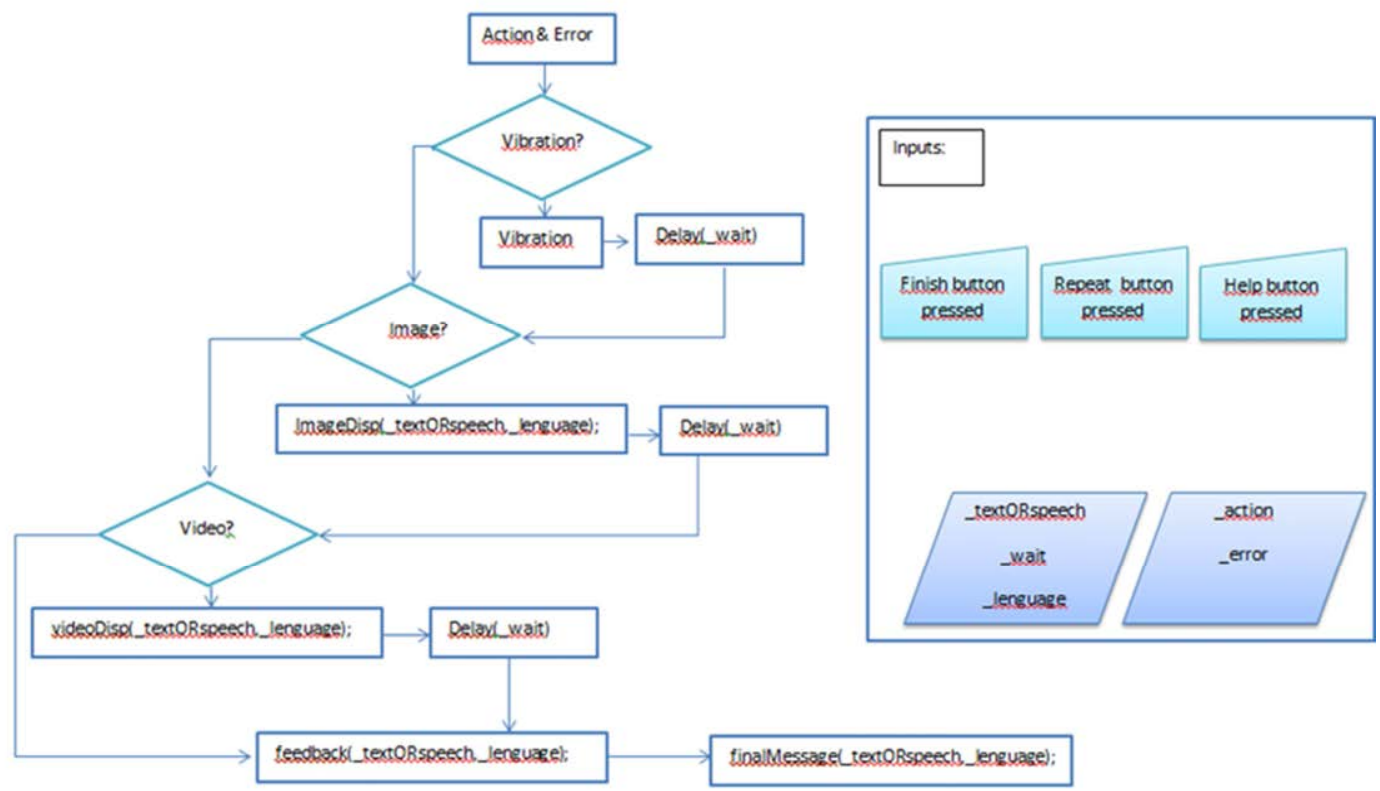

Figure 12. State machine cueing flow diagram.

The outcomes from this evaluation of the system are reported in Table 8 .

Different sequences were evaluated in order to obtain a specific feedback, the output from the system was contrasted with the output from specifications. Notice the discrepancies between task model and the error table, see Table 8. 
Table 8. Results of preliminary evaluation.

\begin{tabular}{|c|c|c|c|c|}
\hline $\begin{array}{l}\text { TASK } \\
\text { TYPE }\end{array}$ & ID & TEST & $\begin{array}{c}\text { Checking } \\
\text { TM }\end{array}$ & $\begin{array}{l}\text { Checking } \\
\text { Error Table }\end{array}$ \\
\hline BT & E01 & $\mathrm{A} 1, \mathrm{~A} 2, \mathrm{~A} 11, \mathrm{~A} 3,[\mathrm{x}],[\mathrm{x}], \mathrm{A} 4,[\mathrm{x}]$ & Yes & Yes \\
\hline BT & $\mathrm{E} 01+\mathrm{E} 18$ & $\mathrm{~A} 1, \mathrm{~A} 2,[\mathrm{x}], \mathrm{A} 7,[\mathrm{x}],[\mathrm{x}], \mathrm{A} 4,[\mathrm{x}]$ & Yes & Yes \\
\hline BTS & E02 & $\mathrm{A} 1, \mathrm{~A} 2,[\mathrm{x}], \mathrm{A} 3,[\mathrm{x}],[\mathrm{x}], \mathrm{A} 4,[\mathrm{x}]$ & Yes & Yes \\
\hline BTS & $\mathrm{E} 02+\mathrm{E} 26$ & $\mathrm{~A} 1, \mathrm{~A} 2,[\mathrm{x}], \mathrm{A} 3,[\mathrm{x}],[\mathrm{x}], \mathrm{A} 6,[\mathrm{x}]$ & Yes & Yes \\
\hline WTS & E03 E26 & $\mathrm{A} 1, \mathrm{~A} 2, \mathrm{~A} 6$ & Yes & Yes \\
\hline WT & E04 & $\mathrm{A} 1, \mathrm{~A} 2, \mathrm{~A} 10$ & Yes & Yes \\
\hline WTS & E05 & $\mathrm{A} 1, \mathrm{~A} 2,[\mathrm{x}], \mathrm{A} 3,[\mathrm{x}],[\mathrm{x}], \mathrm{A} 4,[\mathrm{x}]$ & Yes & No \\
\hline WTS & $\mathrm{E} 05+\mathrm{E} 18$ & $\mathrm{~A} 1, \mathrm{~A} 2,[\mathrm{x}], \mathrm{A} 7,[\mathrm{x}],[\mathrm{x}], \mathrm{A} 4,[\mathrm{x}]$ & Yes & Yes \\
\hline BTS & E13 & $\mathrm{A} 1, \mathrm{~A} 2,[\mathrm{x}], \mathrm{A} 3,[\mathrm{x}],[\mathrm{x}]$ & Yes & Yes \\
\hline WTS & E22 & $\begin{array}{c}\mathrm{A} 1, \mathrm{~A} 2, \mathrm{~A} 3, \mathrm{~A} 4, \mathrm{~A} 5, \mathrm{~A} 6, \mathrm{~A} 7, \mathrm{~A} 8 \\
\mathrm{~A} 7\end{array}$ & Yes & No \\
\hline WT & E21 & $\mathrm{A} 1, \mathrm{~A} 2, \mathrm{~A} 3, \mathrm{~A} 4, \mathrm{~A} 6, \mathrm{~A} 7, \mathrm{~A} 8, \mathrm{~A} 7$ & Yes & No \\
\hline BT & No errors & $\mathrm{A} 1, \mathrm{~A} 2, \mathrm{~A} 3, \mathrm{~A} 4, \mathrm{~A} 8, \mathrm{~A} 7, \mathrm{~A} 7$ & Yes & Yes \\
\hline BT & E10 & $\mathrm{A} 1, \mathrm{~A} 2, \mathrm{~A} 2, \mathrm{~A} 3, \mathrm{~A} 2, \mathrm{~A} 2$ & Yes & Yes \\
\hline BT & $\mathrm{E} 10+\mathrm{E} 18+(\mathrm{E} 10)$ & $\mathrm{A} 1, \mathrm{~A} 2, \mathrm{~A} 2, \mathrm{~A} 7, \mathrm{~A} 2, \mathrm{~A} 3, \mathrm{~A} 2$ & Yes & No \\
\hline WT & E12 & $\mathrm{A} 3, \mathrm{~A} 1, \mathrm{~A} 2, \mathrm{~A} 3$ & Yes & Yes \\
\hline WT & $\mathrm{E} 12+\mathrm{E} 01$ & $\mathrm{~A} 1, \mathrm{~A} 2, \mathrm{~A} 3,[\mathrm{x}], \mathrm{A} 3$ & Yes & Yes \\
\hline BTS & E13 & $\mathrm{A} 1, \mathrm{~A} 2, \mathrm{~A} 3, \mathrm{~A} 4, \mathrm{~A} 9$ & Yes & Yes \\
\hline BTS & $\mathrm{E} 13+\mathrm{E} 05$ & $\mathrm{~A} 1, \mathrm{~A} 2,[\mathrm{x}], \mathrm{A} 3,[\mathrm{x}],[\mathrm{x}], \mathrm{A} 9$ & Yes & Yes \\
\hline WTS & E14 & $\mathrm{A} 1, \mathrm{~A} 3, \mathrm{~A} 4$ & Yes & Yes \\
\hline WTS & $\mathrm{E} 14+\mathrm{E} 27$ & $\mathrm{~A} 1, \mathrm{~A} 6, \mathrm{~A} 3, \mathrm{~A} 6, \mathrm{~A} 4$ & Yes & Yes \\
\hline BT & E15 & $\begin{array}{c}\mathrm{A} 1, \mathrm{~A} 2, \mathrm{~A} 4, \mathrm{~A} 4, \mathrm{~A} 4, \mathrm{~A} 3, \mathrm{~A} 7, \mathrm{~A} 4 \\
\mathrm{~A} 4, \mathrm{~A} 4\end{array}$ & Yes & No \\
\hline WT & E18 & $\mathrm{A} 7, \mathrm{~A} 1, \mathrm{~A} 2, \mathrm{~A} 7, \mathrm{~A} 7$ & Yes & Yes \\
\hline BT & E20 & $\begin{array}{c}\mathrm{A} 3, \mathrm{~A} 1, \mathrm{~A} 2, \mathrm{~A} 4, \mathrm{~A} 7, \mathrm{~A} 7, \mathrm{~A} 7, \mathrm{~A} 7 \\
\mathrm{~A} 7\end{array}$ & Yes & No \\
\hline BTS & E21 & $\begin{array}{c}\mathrm{A} 3, \mathrm{~A} 1, \mathrm{~A} 5, \mathrm{~A} 2, \mathrm{~A} 4, \mathrm{~A} 7, \mathrm{~A} 7, \mathrm{~A} 7 \\
\mathrm{~A} 7, \mathrm{~A} 7, \mathrm{~A} 7\end{array}$ & Yes & No \\
\hline WTS & E22 & $\begin{array}{c}\mathrm{A} 3, \mathrm{~A} 1, \mathrm{~A} 5, \mathrm{~A} 2, \mathrm{~A} 4, \mathrm{~A} 6, \mathrm{~A} 7, \mathrm{~A} 7 \\
\mathrm{~A} 7, \mathrm{~A} 7, \mathrm{~A} 7, \mathrm{~A} 7\end{array}$ & Yes & No \\
\hline WT & E23 & A5 & Yes & Yes \\
\hline BTS & $\mathrm{E} 24$ & $\mathrm{~A} 3, \mathrm{~A} 1, \mathrm{~A} 5, \mathrm{~A} 2, \mathrm{~A} 4, \mathrm{~A} 7, \mathrm{~A} 5$ & Yes & Yes \\
\hline BT & $\mathrm{E} 23$ & $\mathrm{~A} 3, \mathrm{~A} 1, \mathrm{~A} 2, \mathrm{~A} 4, \mathrm{~A} 7, \mathrm{~A} 6$ & Yes & Yes \\
\hline WTS & $\mathrm{E} 27$ & $\begin{array}{c}\mathrm{A} 3, \mathrm{~A} 6, \mathrm{~A} 1, \mathrm{~A} 5, \mathrm{~A} 6, \mathrm{~A} 2, \mathrm{~A} 4, \mathrm{~A} 6 \\
\mathrm{~A} 7, \mathrm{~A} 6, \mathrm{~A} 6\end{array}$ & Yes & Yes \\
\hline WTS & $\mathrm{E} 27+\mathrm{E} 07$ & $\begin{array}{c}\mathrm{A} 3, \mathrm{~A} 6, \mathrm{~A} 1, \mathrm{~A} 5, \mathrm{~A} 1, \mathrm{~A} 6, \mathrm{~A} 2, \mathrm{~A} 4 \\
\mathrm{~A} 6, \mathrm{~A} 7, \mathrm{~A} 6, \mathrm{~A} 6\end{array}$ & Yes & Yes \\
\hline BTS & E23 & $\mathrm{A} 3, \mathrm{~A} 5, \mathrm{~A} 2$ & Yes & Yes \\
\hline WT & E30 & $\mathrm{A} 1, \mathrm{~A} 3, \mathrm{~A} 8$ & Yes & Yes \\
\hline WTS & $\mathrm{C} 01$ & $\mathrm{~A} 1, \mathrm{~A} 2, \mathrm{~A} 3, \mathrm{~A} 4, \mathrm{~A} 5, \mathrm{~A} 6, \mathrm{~A} 7, \mathrm{~A} 8$ & Yes & Yes \\
\hline BTS & $\begin{array}{c}\mathrm{C} 01+\mathrm{E} 01+\mathrm{E} 02+ \\
\mathrm{E} 05\end{array}$ & $\begin{array}{c}\mathrm{A} 1, \mathrm{~A} 2, \mathrm{~A} 4,[\text { help] }, \mathrm{A} 3, \text { [pause], } \\
\text { [finish], A5, A7, A8 }\end{array}$ & Yes & Yes \\
\hline
\end{tabular}


The poor results obtained, especially in the case of quantitative actions, were manifested by failures in the output expected from the feedback system.

As a result of generalization, dealing with complex models may result in greater complexity on definition of errors. The best solution in these cases is to use multiple models of the system allowing specialization in the task. For example, in the task making a cup of tea the general model was divided into different models within categories only tea, tea with milk, tea with sugar and tea with milk and sugar. In this way it was possible to create sub models task in which it is established whether they were mistakes or were not, certain actions depending on patient preferences.

From the observation of the user it can be clustered, via data mining, the group of sequence which best generalise the different kind of ways of performing a task. In Table 9, the different sequence chosen for the task of making a tea are represented as an example. See appendix F, where caption labels are described.

Table 9. Available sequence for tea making.

\begin{tabular}{|l|}
\hline Black tea: \\
\hline BT_01 : $\{\mathrm{A} 1, \mathrm{~A} 2, \mathrm{~A} 3, \mathrm{~A} 4, \mathrm{~A} 7, \mathrm{~A} 8\}$ \\
\hline BT_wrt2 $:\{\mathrm{A} 1, \mathrm{~A} 2, \mathrm{~A} 3, \mathrm{~A} 4, \mathrm{~A} 7\}$ \\
\hline Black tea with sugar: \\
\hline BTS_01 : $\{\mathrm{A} 1, \mathrm{~A} 2, \mathrm{~A} 3, \mathrm{~A} 5, \mathrm{~A} 4, \mathrm{~A} 7, \mathrm{~A} 8, \mathrm{~A} 7\}$ \\
\hline BTS_02 $:\{\mathrm{A} 1, \mathrm{~A} 2, \mathrm{~A} 3, \mathrm{~A} 4, \mathrm{~A} 8, \mathrm{~A} 5, \mathrm{~A} 7\}$ \\
\hline BTS_wrt2 : $\{\mathrm{A} 1, \mathrm{~A} 2, \mathrm{~A} 3, \mathrm{~A} 4, \mathrm{~A} 5, \mathrm{~A} 7\}$ \\
\hline White tea: \\
\hline WT_01 : $\{\mathrm{A} 1, \mathrm{~A} 2, \mathrm{~A} 3, \mathrm{~A} 4, \mathrm{~A} 7, \mathrm{~A} 8, \mathrm{~A} 6, \mathrm{~A} 7\}$ \\
\hline WT_03 : $\{\mathrm{A} 1, \mathrm{~A} 2, \mathrm{~A} 3, \mathrm{~A} 6, \mathrm{~A} 4, \mathrm{~A} 7, \mathrm{~A} 8\}$ \\
\hline WT_wrt2 $:\{\mathrm{A} 1, \mathrm{~A} 2, \mathrm{~A} 3, \mathrm{~A} 4, \mathrm{~A} 7, \mathrm{~A} 6, \mathrm{~A} 7\}$ \\
\hline White tea with sugar: \\
\hline WTS_01 : $\{\mathrm{A} 1, \mathrm{~A} 2, \mathrm{~A} 3, \mathrm{~A} 4, \mathrm{~A} 8, \mathrm{~A} 6, \mathrm{~A} 5, \mathrm{~A} 7\}$ \\
\hline WTS_03 : $\{\mathrm{A} 1, \mathrm{~A} 2, \mathrm{~A} 3, \mathrm{~A} 5, \mathrm{~A} 4, \mathrm{~A} 6, \mathrm{~A} 7, \mathrm{~A} 8\}$ \\
\hline WTS_wrt1 : $\{\mathrm{A} 1, \mathrm{~A} 2, \mathrm{~A} 3, \mathrm{~A} 5, \mathrm{~A} 6, \mathrm{~A} 4, \mathrm{~A} 8, \mathrm{~A} 7\}$ \\
\hline WTS_wrt2 : $\{\mathrm{A} 1, \mathrm{~A} 2, \mathrm{~A} 3, \mathrm{~A} 4, \mathrm{~A} 6, \mathrm{~A} 5, \mathrm{~A} 7\}$ \\
\hline
\end{tabular}


The problem now is to determine the best available sequence for each user. The idea beneath the problem is to find the combination of words in a dictionary which most approximate to the given one. This problem is better known as a "closest string" problem, when the size of the string (number of characters) can vary the problem is known as "closest substring".

A possible solution has been evaluated. First, it is necessary to describe the hamming distance as the number of not coincidence characters between two words of the same length. Now it can be defined the problem of the closest string, given a set of strings each of length $\mathrm{m}$, the closest string problem is to find the smallest $\mathrm{d}$ and a string $\mathrm{s}$ of length $\mathrm{m}$ which is within Hamming distance $d$ to each element of the dictionary. This problem comes from coding theory when we are looking for a code not too far away from a given set of codes and is NP hard. The closest substring problem, with an additional input integer L, asks for the smallest $d$ and a string s, of length $\mathrm{L}$, which is within Hamming distance d away from a substring, of length L, of each substring in a dictionary D. This problem is much more elusive than the closest string problem. The Closest Substring problem is formulated from applications in finding conserved regions, identifying genetic drug targets and generating genetic probes in molecular biology.

To exemplify this problem, given the following sequences: A: $\{\mathrm{A} 1, \mathrm{~A} 3, \mathrm{~A} 4, \mathrm{~A} 5\}$ and $\mathrm{B}:\{\mathrm{A} 2, \mathrm{~A} 3, \mathrm{~A} 1, \mathrm{~A} 4, \mathrm{~A} 5\}$, the closest substring is described as the minimum set of actions necessary to convert the second sequence (B) into the first one (A) by describing a finite set of possible actions listed below, see Table 10. Set of actions in closest sub-string problem. Table 10:

Table 10. Set of actions in closest sub-string problem.

\begin{tabular}{|ll|}
\hline Action & Example \\
\hline Insertion & $\{\mathrm{A} 1, \mathrm{~A} 2\} \rightarrow\{\mathrm{A} 1, \mathrm{~A} 2, \mathrm{~A} 3\}$ \\
Deletion & $\{\mathrm{A} 1, \mathrm{~A} 2, \mathrm{~A} 3, \mathrm{~A} 4\} \rightarrow\{\mathrm{A} 1, \mathrm{~A} 2, \mathrm{~A} 3\})$ \\
Substitution & $\{\mathrm{A} 1, \mathrm{~A} 2, \mathrm{~A} 4\} \rightarrow\{\mathrm{A} 1, \mathrm{~A} 2, \mathrm{~A} 3\}$ \\
Transposition & $\{\mathrm{A} 1, \mathrm{~A} 3, \mathrm{~A} 2\} \rightarrow\{\mathrm{A} 1, \mathrm{~A} 2, \mathrm{~A} 3\})$ \\
\hline
\end{tabular}


This problem was solved by F.J. Damerau [147] and V.I. Levenshtein [148] who defined the Damerau-Levenshtein distance. A program implementing D-L distance has been developed; the program allows the user to change the weighs of each of the actions to penalize for example insertion actions against substitution actions which are directly related with human cognitive errors because the words of the dictionary are representing subtask in a multi -step sequential activity.

\subsubsection{Model checking}

Regarding quantitative errors, the problem is that, including the number of repetitions of an action increases dimensionality of the problem with the possibility of making unfeasible to deal with it using exact methods. Limitations of the Markov based task model could be bootstrapped with a model checking strategy. As reported in [7, Ch. 17], "The main advantage of planning by model checking is the ability to plan under uncertainty in a practical way. Non determinism leads to the need to deal with different action outcomes". Following this philosophy an additional profile module was added to deal with the problems discussed in the previous section. The following figure, Figure 13, details the interaction between the module based on MDP and the plug required to solve this problem. This module kills two birds with one stone, as it facilitates the processing of information in a process of concurrent track structure described in the next chapter, chapter 4 .

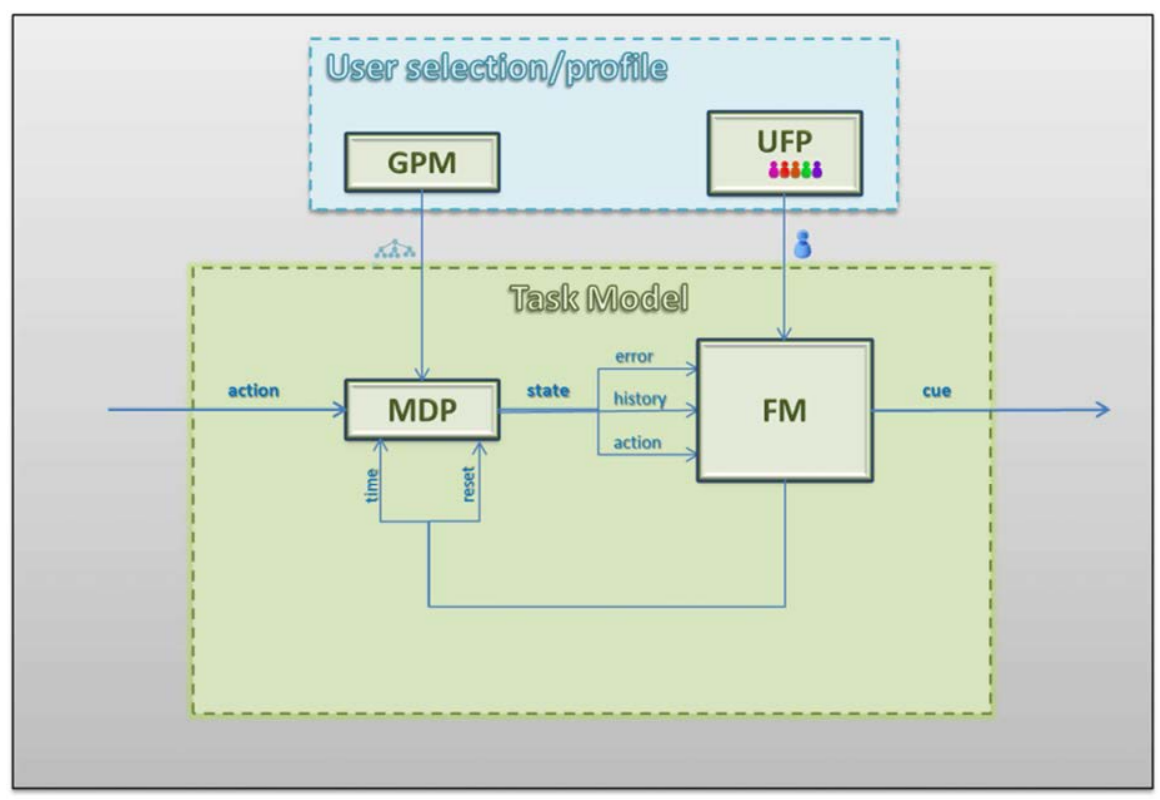


Figure 13. MDP module - Profile module interactions.

The profile module complements the MDP based module via:

- Providing personalization presenting cues in accordance with user preferences.

- Avoiding the dimensional exploitation of the graph with repetitive actions.

- Enhancing other solutions avoiding truncations to virtual states which end in unrealistic scenarios.

- Dealing with quantitative errors ignoring the requests of the planning system until the individualised model suggests so. The request can be heard or ignored increasing the universe of discussion.

- Planning different cueing strategies in accordance with the level of expertise of the user.

\subsection{Functional description of the system}

These section aims at covering the implementation of the system in terms of architecture and functionalities. The general architecture is defined as a three level architecture and is based on ISO/IEC/IEEE 42010 [149]. The description of the software can be split into a logic module and two communication modules which handle the inputs and outputs, see Figure 14. In this section the functional characteristics of each module are described. Additionally, some communication tests are presented.

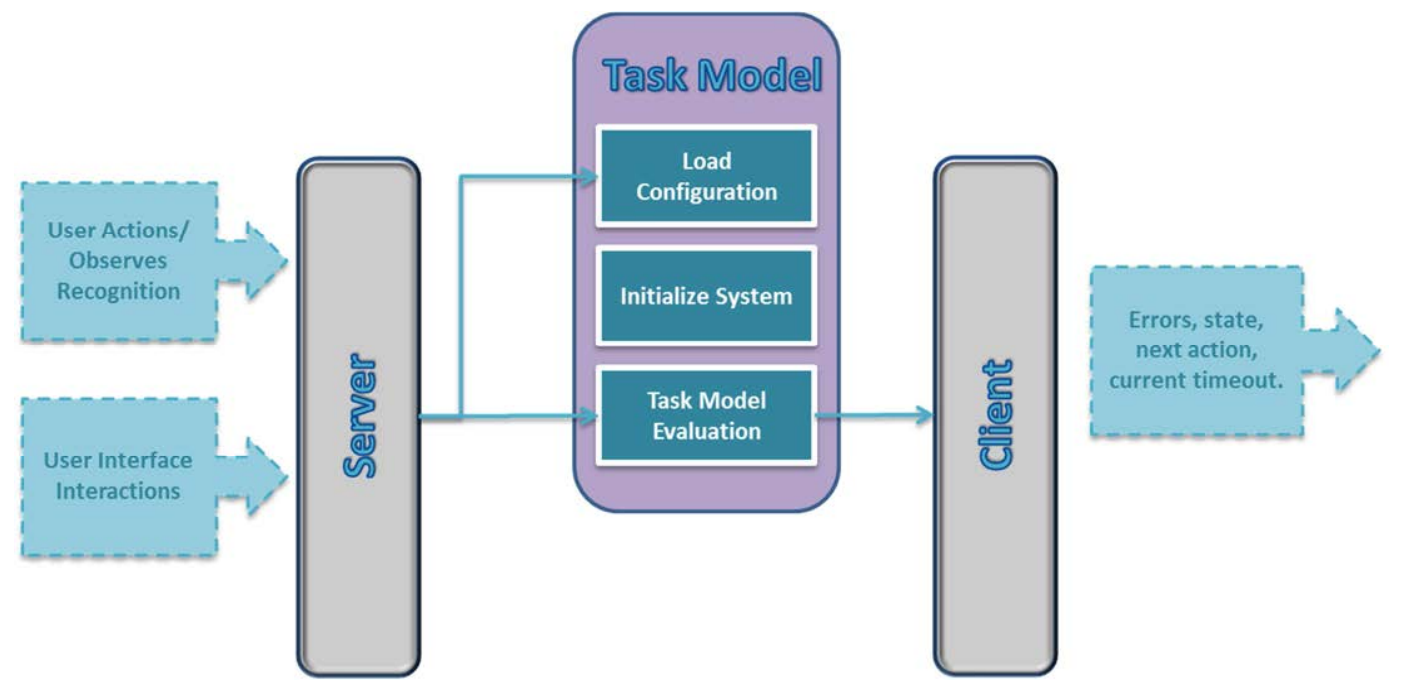

Figure 14. General three level architecture. 


\subsubsection{Central module}

The central module of this system is in charge of controlling the information workflow and providing the logic, see Figure 15.

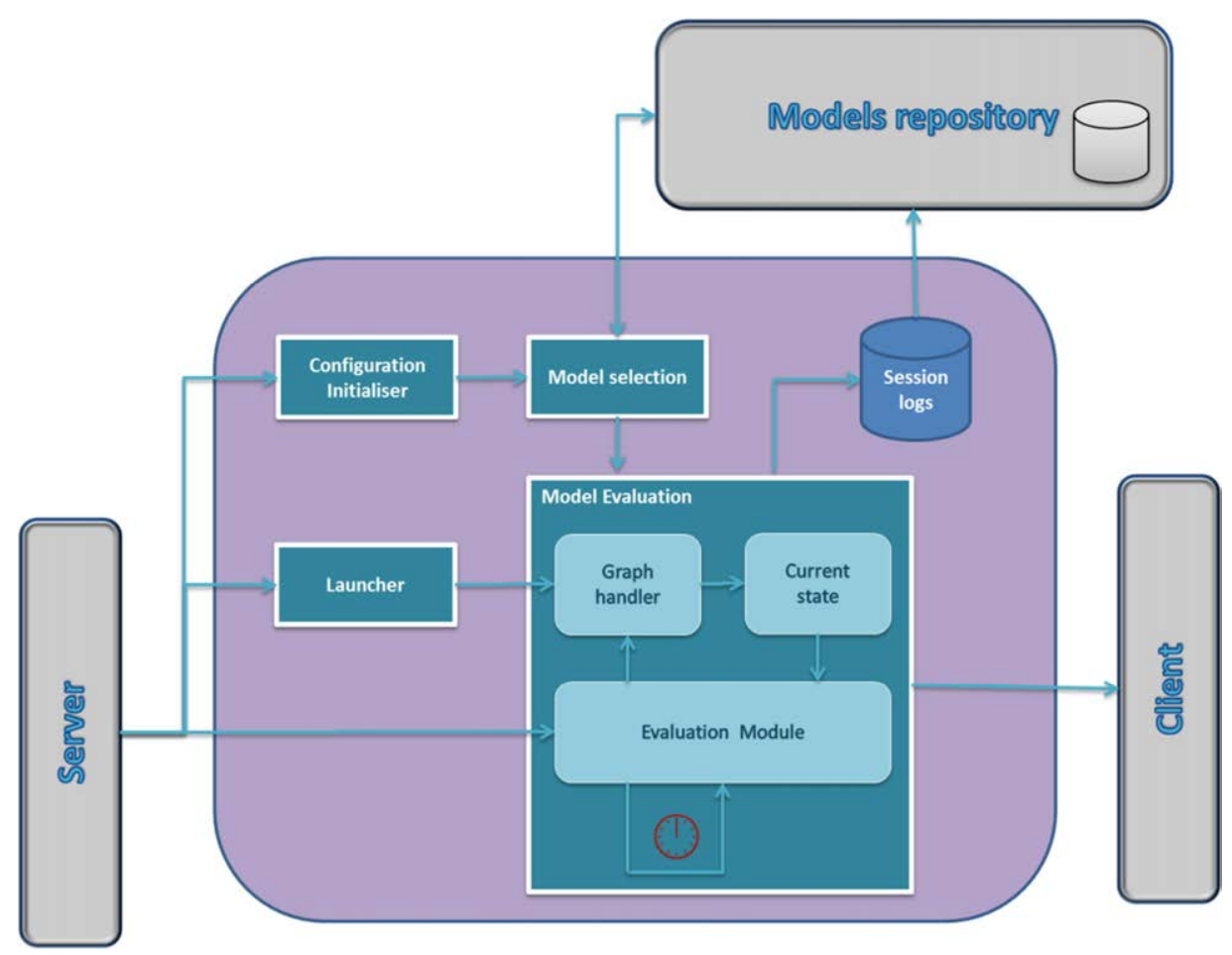

Figure 15. Description of the inner logic.

The modules have the following functionalities:

\section{Configuration Initializer:}

The configuration initializer is in charge of setting the task and finds the most appropriate model for the user.

\section{Model Selector:}

The Model Selector loads the selected model from the Models Repository. This model is used to construct the Graph Handler.

\section{Launcher:}

The Launcher is in charge of handling the GUI interactive actions such as pressing start, stop, repeat or pause. The module is also in charge of setting the initial state. 


\section{Graph Handler:}

The Graph Handler is programmed as a double (forward/backward) linked tree. Where each node represents a state object with the properties described below:

- Type of state: Indicates if the state is terminal, normal or the target state. Also, if contains direct transition to other states to avoid internal loops.

- Best next activity: the activity to suggest if the user request or commits a perplexity error.

- Timeout: the time to count for providing the next perplexity error if no action is detected.

- Children nodes: the list of children nodes according to each possible correct action to perform.

- Parent node: the predecessor node in the tree.

- Associated error: the error (if exist) committed for visiting the state.

- Omission error: the error committed if the user considers that task is finished.

\section{Current State:}

This module stores the current state according to the transitions in the tree.

\section{Evaluation Module:}

This is the core module which deals with all the possible interactions and generates the outputs from the system:

- If an action is received from the input buffer evaluates the result from performing that actions in the current state.

- If the perplexity timer expires it is responsible for evaluating the callback routine and return the more suitable action to perform

- If the user considers that the task has been finished the evaluation module checks it. 


\section{Sessions Repository:}

The sessions are stored to use the information of errors to refine the models and inform the supervisor.

\subsubsection{Communication modules}

This part of the architecture is critical in terms of integrations and therefore it is necessary to justify the solution adopted for the communication layer. Integrability is defined as the capacity of a program to be re-used in different contexts or projects. The definition of the inputs and outputs of the developed modules are described in this section. Furthermore, some tests to guarantee the correct performance of the implemented system as a whole has been done and reported at the end of this section.

There are different ways for communicating the Task Model, coded in python language, and a hypothetical module which requires interaction with this module that will be denoted as Information Handler (IH) hereinafter:

- Use of JSON-RPC/XML-RPC.

JSON is a remote procedure call protocol encoded in JSON. It is a very simple protocol, defining only a handful of data types and commands. JSON-RPC allows for notifications and for multiple calls to be sent to the server which may be answered out of order. $\mathrm{XML}-\mathrm{RPC}$ is also a remote procedure call protocol but in this case encoded in XML, currently is better known as SOAP.

- Use of wrappers/ parsers.

Iron python and python for .NET are two brother projects that aim to join the python programming languages with the large amount of functionalities and graphical supports from .NET. It is important to highlight that Iron python was maintained by a small team at Microsoft until the 2.7 Beta 1 release; Microsoft abandoned IronPython in late 2010.

- Inter Process Communication (IPC) 
The most common solution for sharing information between different programming languages is the use of POSIX/WIN32 API functions. Some of these mechanisms are: memory map files, named pipes and sockets. The advantage from this solution is that it is platform and programming language independence.

For the communication module a solution based on POSIX/WIN32 API was chosen. The communication between these modules is bidirectional. A diagram of the information flow is shown in Figure 16

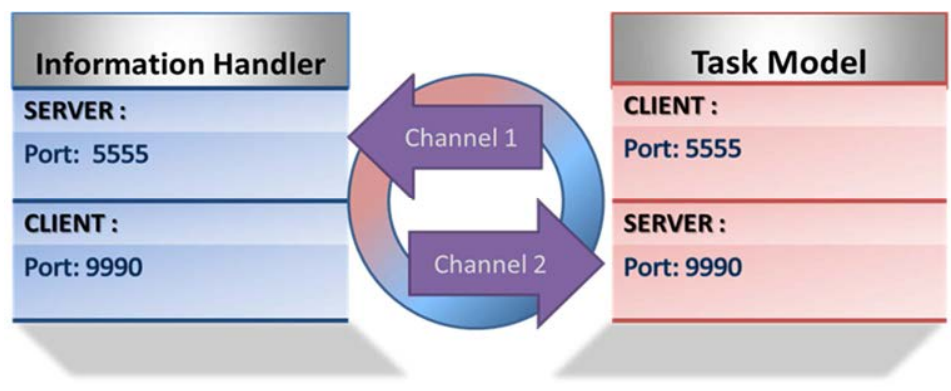

Figure 16. Connection diagram.

Channel 1 is comprised by all the possible codified inputs defined by the TM. Channel 2 is comprised by the inputs for IH. See Figure 17.

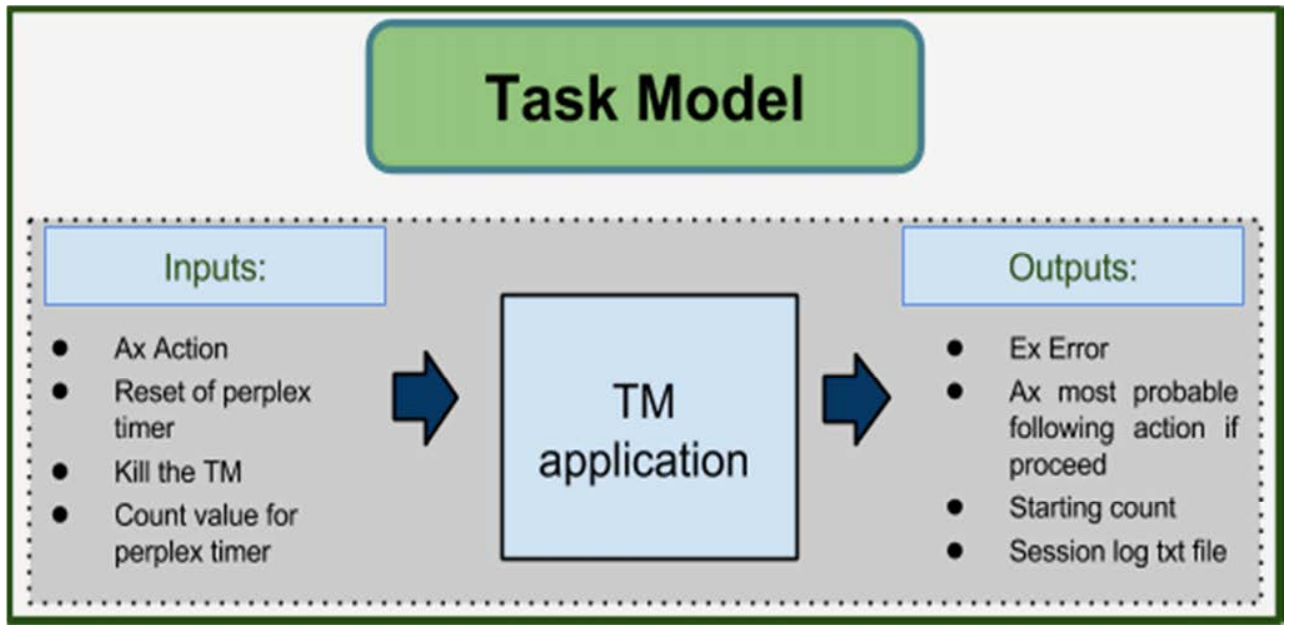

Figure 17. Inputs and outputs diagram.

The use of two channels is justified by the nature of some inputs of the TM. These inputs are temporally undefined and correspond to events triggered by the user, i.e. "pressing a button". To avoid collisions and delays in the information flow these solution is acceptable. 
Two tests have been carried out to determine the suitability of the system. The results and code of these tests are described in Appendix C.

\section{Stress test}

Stress tests are an extended and well accepted method for performing load tests. The aim of the test is to put the program on the limit of the system to conclude if the behaviour of the program is acceptable and agrees with its' requirements.

For doing this test the following protocol will be followed. A random message will be send continuously by the client reducing the time between messages according to an exponential curve.

The following code shows the distribution of times used for testing the communication increasing the load. These codes gives one hundred samples of the decremented gap time ending in the limits of the systems' clock rated in one millisecond which was considered an acceptable sample time for human motion capture.

The following figure, Figure 18, represents how much time is waited every time each trial message is sent. The times decrement from one second to three milliseconds.

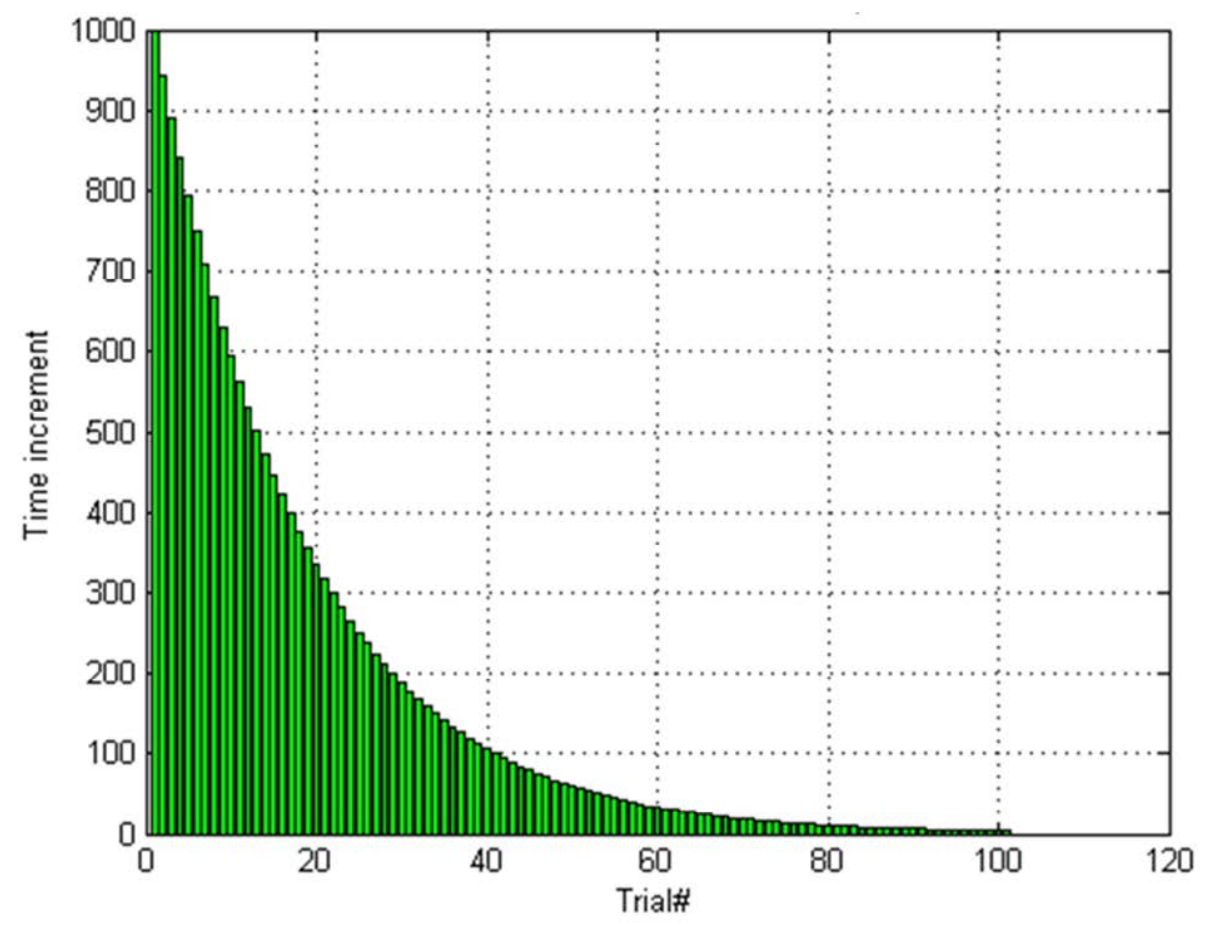


Figure 18. Decrement of times [ms] for each sample.

The load of the system can be expressed as the number of trials by the total amount of time. According to this definition in Figure 19 the total load of the system is represented to give an idea of the test performed.

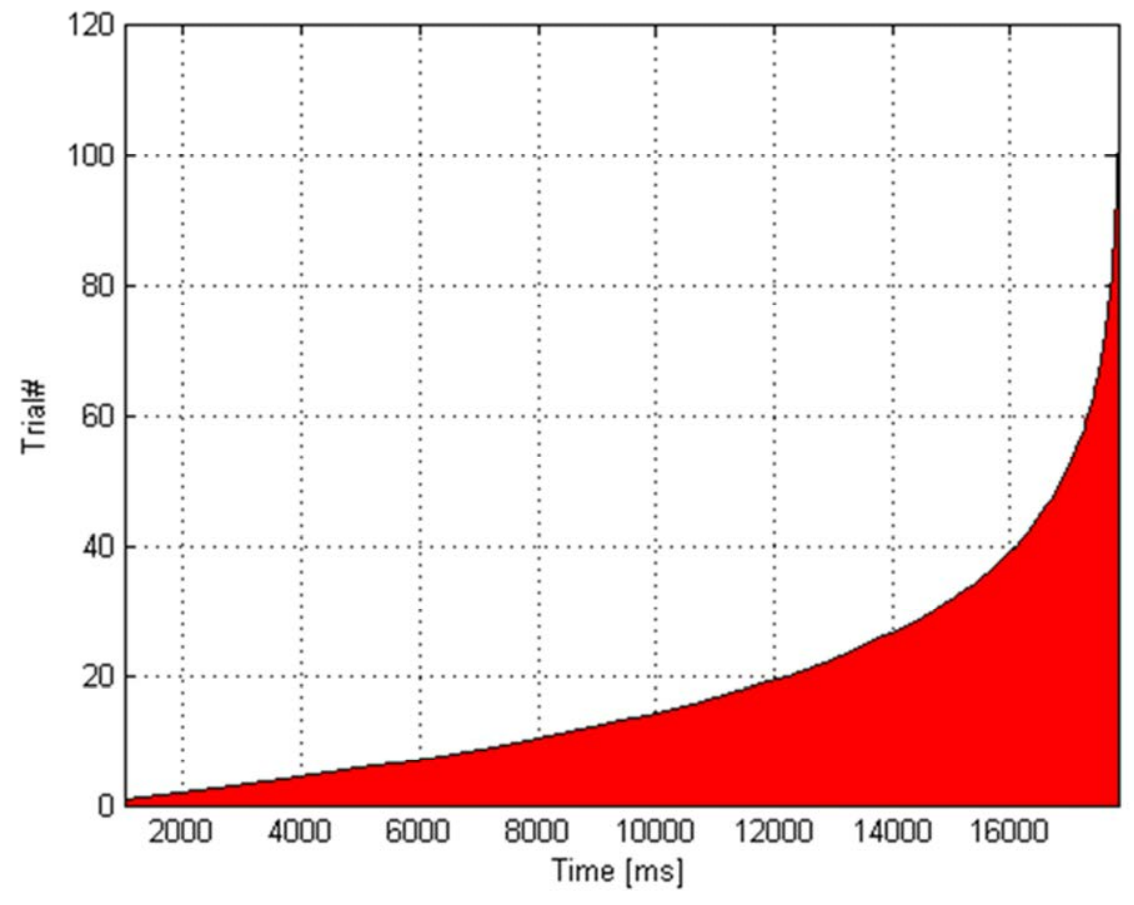

Figure 19.Increment of load in time.

\section{Timing test}

The aim of this test is to measure the delays that the communication via sockets can introduce in the system. Also, time gaps between each trial is sent are reasonable and similar to the smaller ones that the user can spent in performing the fastest subtasks.

The test consist in a continuous information flow of a statistically significance number of trials. During the test timestamps for each message from client and server are collected to measure the delay in the communication. The system clock is the best way to guarantee that both TM and IH times are synchronized and the affordable precision is 1 millisecond, under that time the delay cannot be measured and will be consider insignificant for an interprocess communication based solution. 


\subsection{Developed tools for system management}

One of the main objectives of this thesis is to adapt the tools available to improve and facilitate the transfer of the knowledge from the professional to the system. The following contributions allow the professional to design, in a simple way, a model of common tasks and errors according to their experience. These changes also represent an improvement based on user needs and habits obtaining a more flexible system. To achieve this objective it has been added two tools designed to assist the professional. These tools are the Model Designer and the Cue Designer.

\subsubsection{Model designer}

The Model Designer is a tool for assisting the professional and generates a model of a task. The procedure is explained bellow with the same example from section 0 .

First the program asks the user for the list of subtasks which comprises the task to model, see Figure 20. The methodology to obtain this list is described in 3.2.2. The activities must be described including a representative base time for their execution that will be used later by the decision algorithms.

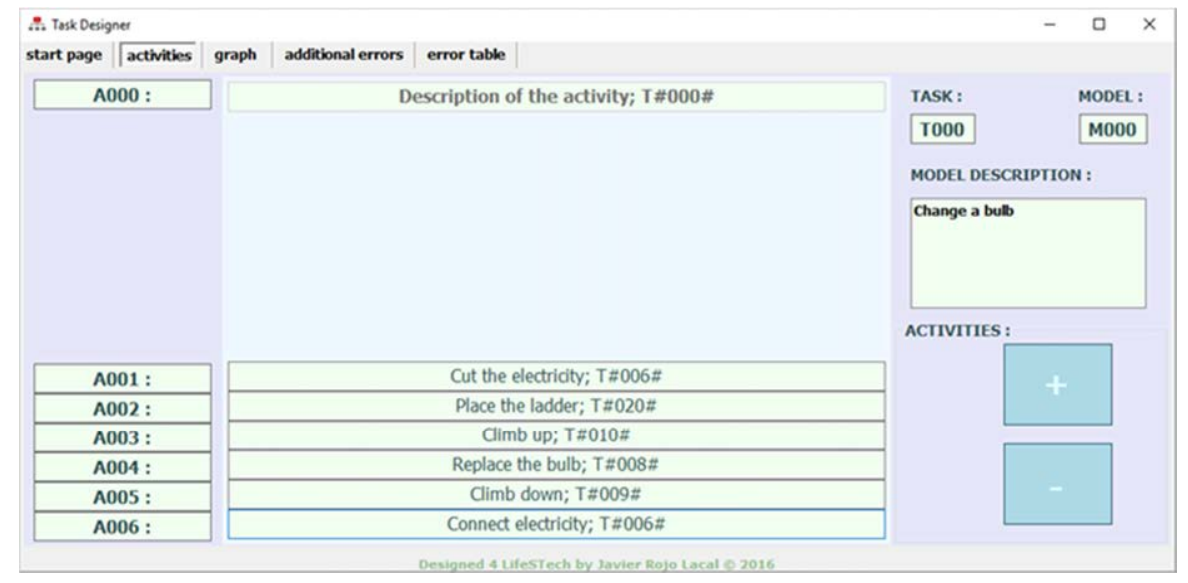

Figure 20. Activities input form.

Then, the application lets the user exploit the probabilistic graphical tree through a GUI, see Figure 21. 


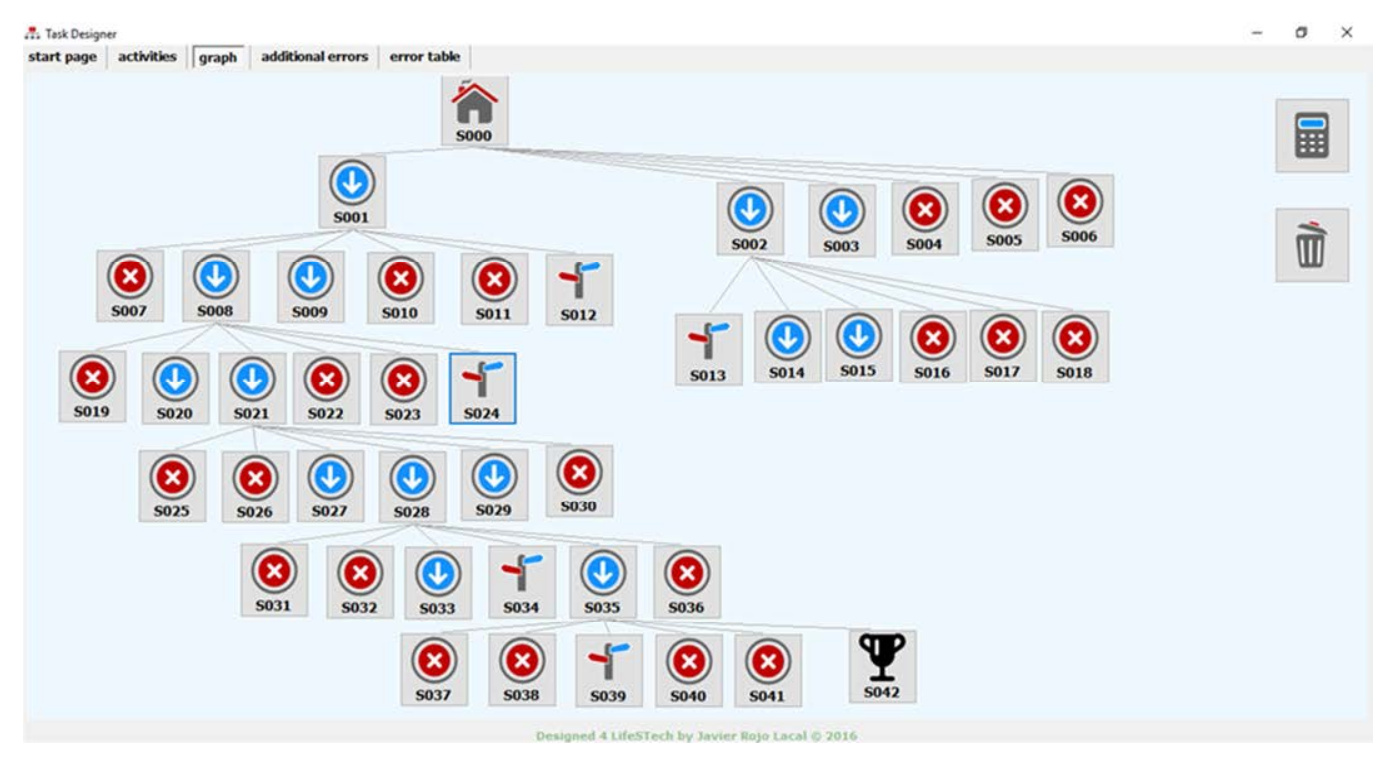

Figure 21.Creation of a probabilistic graphical tree.

Each state can be manipulated to modify their internal properties: transition probabilities between states, typology, reward and the associated errors, see Figure 22 .

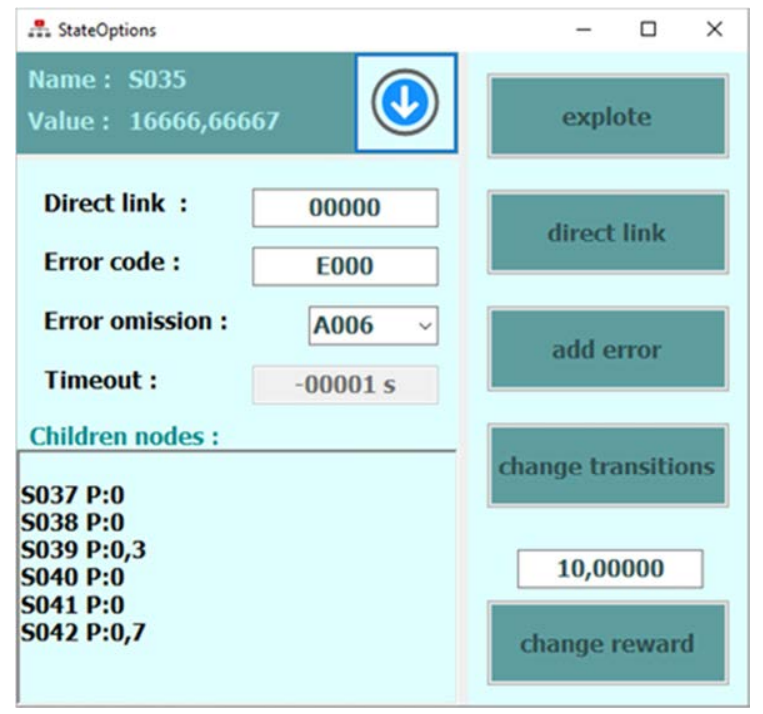

Figure 22. State definition.

Once the probabilistic graphical tree is created and each state defined the program generates the model for the Graph Handler including the timeouts and the planning strategies.

The next step is to add manually the activities with inherent errors that are excluded from the graph to avoid dimensional overflow. In the example, 
breaking bulb constitutes and error. In Figure 23 some of these activities are shown.

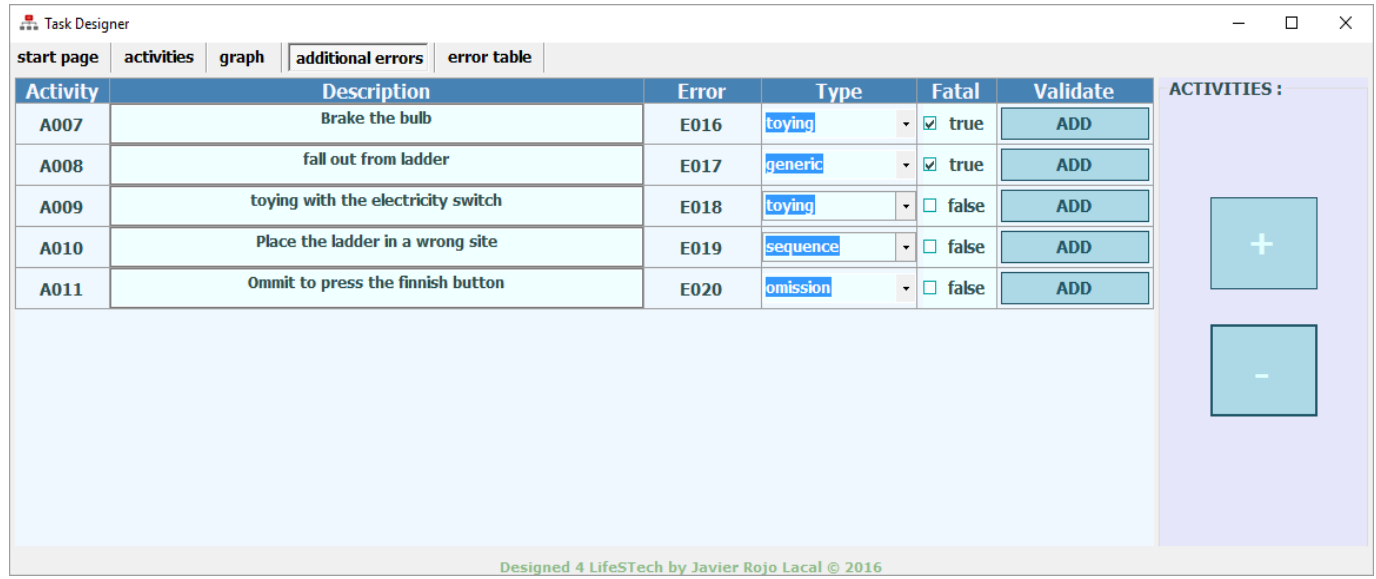

Figure 23.Error activities.

Finally, the program generates the error list and lets the user save the model for the task, see Figure 24. Some examples of errors tables can be found in Appendix F.

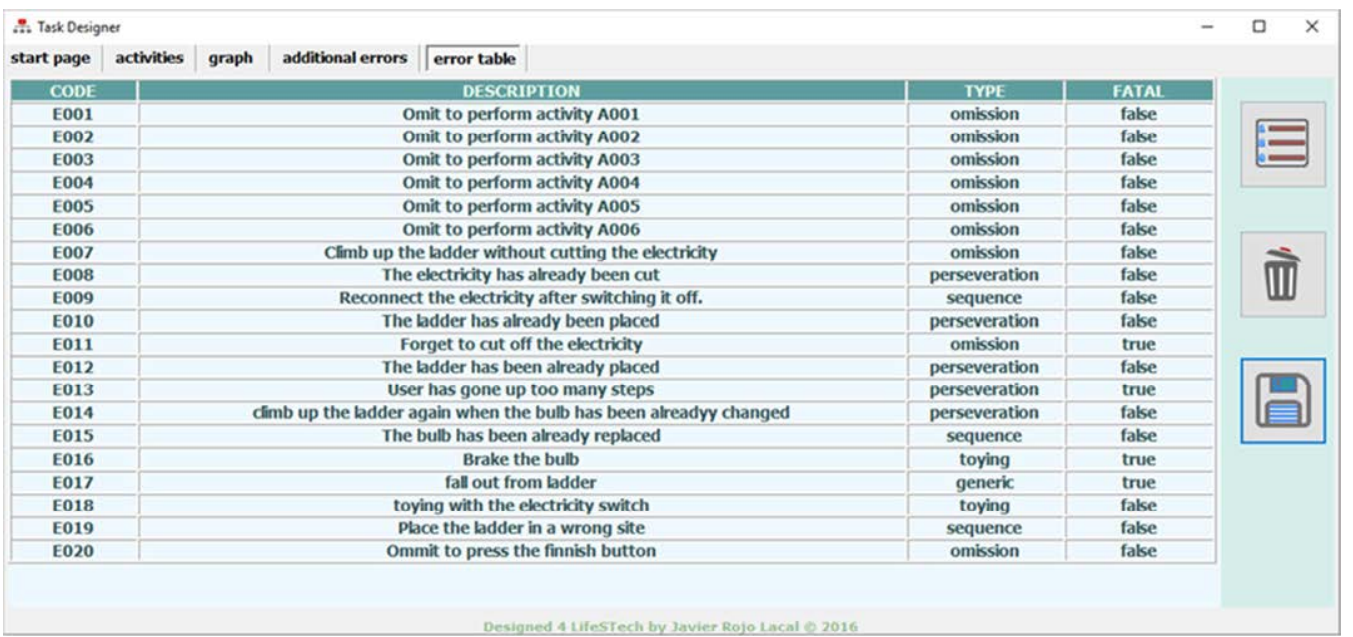

Figure 24.Error list generated.

\subsubsection{Cue Designer}

To assist the professional, a valuable tool has been developed. The tool is a program which allows the professional to adapt the structure of the cueing system. The Figure 25 shows the visual appearance of the program for a defined error table; see Appendix $\mathrm{F}$ for more information on defined error tables. 


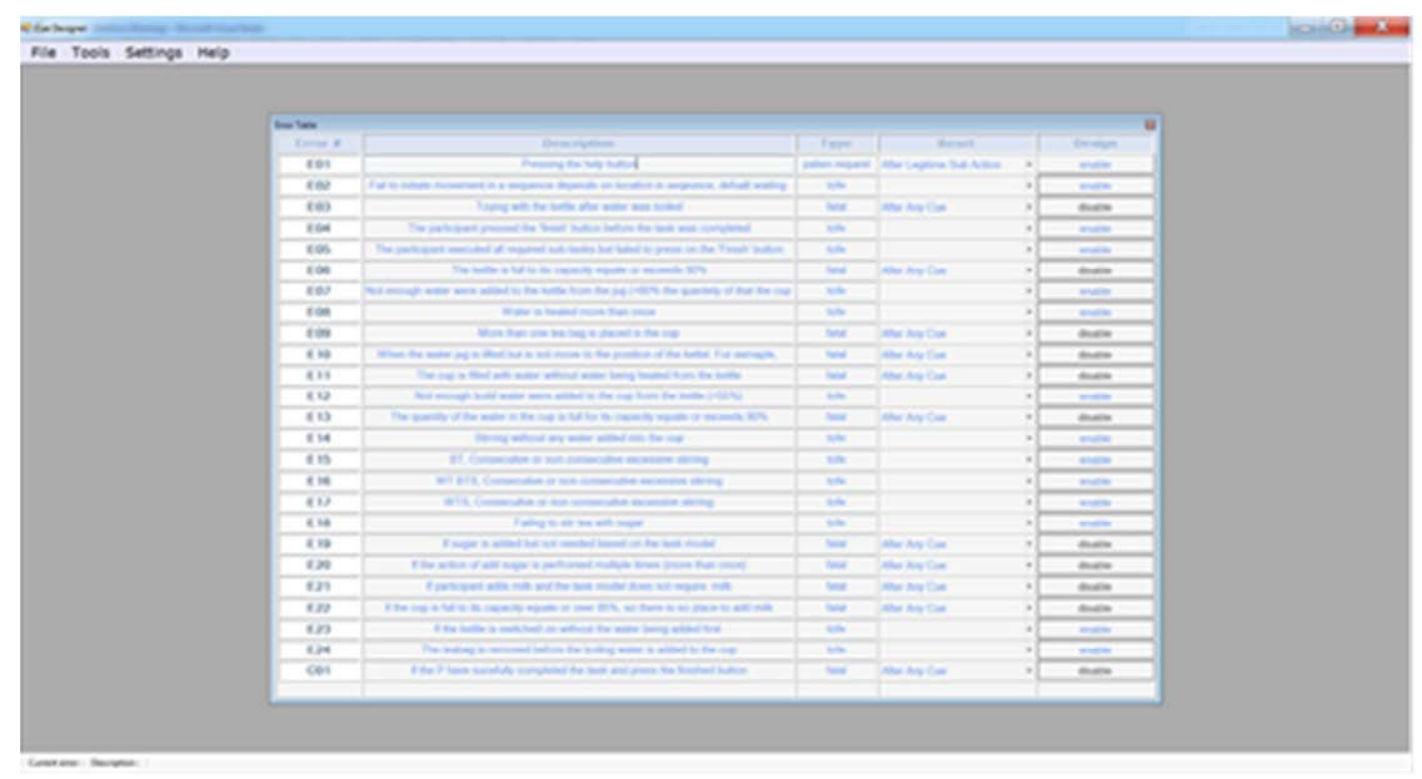

Figure 25. Profile module interface.

The tool generates a structure for supervising MDP-module which stored in an eXtensible Markup Language (XML) file [150], due to ethical implications the file is codified and later encrypted with standard methods with a double asymmetric key [151], all the information about the structure of the process by an error oriented management. The file stores also all the cueing information, described in chapter 4. It makes this solution ideal to manage the performance of different users individually for each defined error. Figure 26 shows the interface for modifying the cue structure.

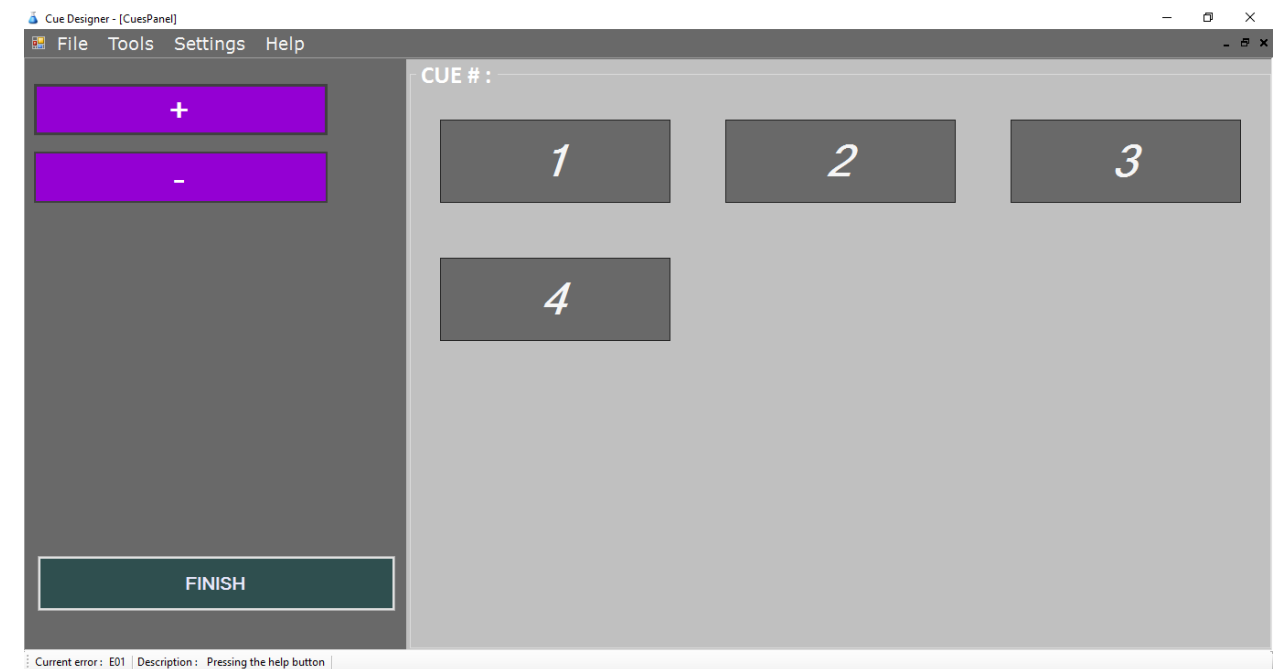

Figure 26. Cue structure definition. 
The local storing strategy is implemented in order to avoid consequences of bad management of data and the use of relational data bases which leads to the problems described in [152] regarding BD.

\subsection{Lessons from Task Modelling}

The methodology described in this chapter includes all the desirable functionalities and properties reviewed in the literature. These properties are segmentation, knowledge from the expert, personalization, modularity, adaptability and integration with other modules. The solution presented avoids the use of abstractions and can be represented by using the expert words and terminology. This makes easier the process of modelling and simplifies the system. In the particular case of the application to the clinical study of post-stoke patients, the specifications reveal a greater willingness to qualitative study of errors. However, a system based only on MDP dealing with quantitative errors with techniques such as Temporal Difference or Value Function Approximation require factorization and abstractions that in some manner affect the output oriented to a guidance system. The use of a hybrid strategy based both on MDP and Model Checking avoid this problematic at the same time as quantitative errors are managed according to the user preferences. Furthermore, the iterative algorithm presented allows a fast resolution of the task graph allowing on the fly resolution if required. 


\section{CHAPTER 4}

\section{Multimodal Reinforcement}

This chapter describes a cue system for assisting users under the circumstances of the work reported on the previous chapter. Some considerations to take into account for a proper assistance are summarized. These considerations have been implemented in a multimodal interface for assisting the user. The interface has been designed taking into consideration the ideas from the literature and the opinion from the experts. It is described the modality of the cues and the way in which the cues are presented to the user. Special attention is taken in the way of extracting information from task model to provide contextualised feedback. At the end of the chapter is reported an experiment to validate the multimodal interface presented in this chapter with some users applied to a learning tasks. The simplicity of the methodology applied in this study makes it useful for the validation of multiple multimodal interfaces. The idea underneath the study is to test if the interface enhances significantly the learning factor. Finally, the outcomes from the study are analysed to manifest the suitability of the system for applying cognitive rehabilitation. The study concludes that the proposed interface lets users finish the execution of the task faster and committing fewer errors when receiving multimodal help through the interactive interfaces instead of training by their own. 


\subsection{Interfaces for learning}

M. Minsky said[153]:

"You don't understand anything until you learn it more than one way".

The quote claims the advocacy of use of alternative strategies to ensure that any new chunk of learned information could be obtained from different sources. It was a main worry from the recently lost genius of Artificial Intelligence (AI). These statements have caused some controversy because Minsky is also considered by some as one of the forerunners of the known "AI-winter" during seventies by promoting driving research away from neural networks. But, letting all that controversy apart, nonconformity to find new ways to learn is what made M. Minsky a genius and, undoubtedly, one of the basis of learning.

Error awareness is another important actor in the regulation of cognitive processes. From conflict monitoring theory, which comprises a model for simulating a task performance and error related negativity, some researches has contrasted the conflict monitoring performing errors and corrections with the brain potentials of Anterior Cingulate Cortex to explain the dynamics of error detection [154]-[157]. An interesting conclusion from these studies is the role of a fast correcting response to enhance self-error perception.5

The use of multimodal interfaces for learning has been extensively studied since the invention of the graphical user interface by J.C.R. Licklider during his work on the SAGE project[158]. Researchers have been focused on the way of improving human computer interaction to transmit information faster and clearer. Previously, the classic study of this subject was constituted by the stimulus-response tests, as reported in the work of behaviourist headed by B.F. Skinner[159], [160]. Classical studies were conceived to improve our Response Times (RT), and, therefore, as previously mentioned, error perception and other factors correlated. But, the fact that stimuli were increasing in complexity made researchers deep into the problematic of compatibility[161] and focus the attention also on categorical perception[162]. The inclusion of new communication channels and the conception of the machine as an extension of human capabilities modify the classical point of 
view. Modality of the cues and their effects has been contrasted in multiple studies, to cite some[163]-[165]. A generalized outcome from these studies is that, usually, the richer the feedback provided to the user, the better performance of the actions and RT. Another rational conclusion from these studies was the proper distribution of the interface layout and the Fitts' law[166]. The problem is that users can always be divided into categorical groups and the stimuli must always be carefully selected to avoid incompatibilities.

Widespread characterization studies of learning are representations of error rates or performance over practice by learning curves.

Learning curves provide a way to measure a cognitive model. A generalize form of modelling learning is the power law of practice postulated by A. Newell and P.S. Rosenbloom[167]. According to this law, practice decreases the error rate in an exponential order. The peaks presented in the learning curve are considered hidden knowledge components. These hidden knowledge blips represent different sub-task which can be split and learned independently in a more likely ratio to the ideal of the power law. Different approximations have been reported since the first analysis of these curves and more complex methodologies has been presented to analyse the learning factor to evaluate a cognitive model[168]. The next stage is to categorize the results in order to apply the assumptions of each model.

Regarding the system attention the authors M. Solhberg and C. Mateer described in [169] the guidelines for improving attention based on the model of skill acquisition. The following figure shows the main points from these guidelines. 


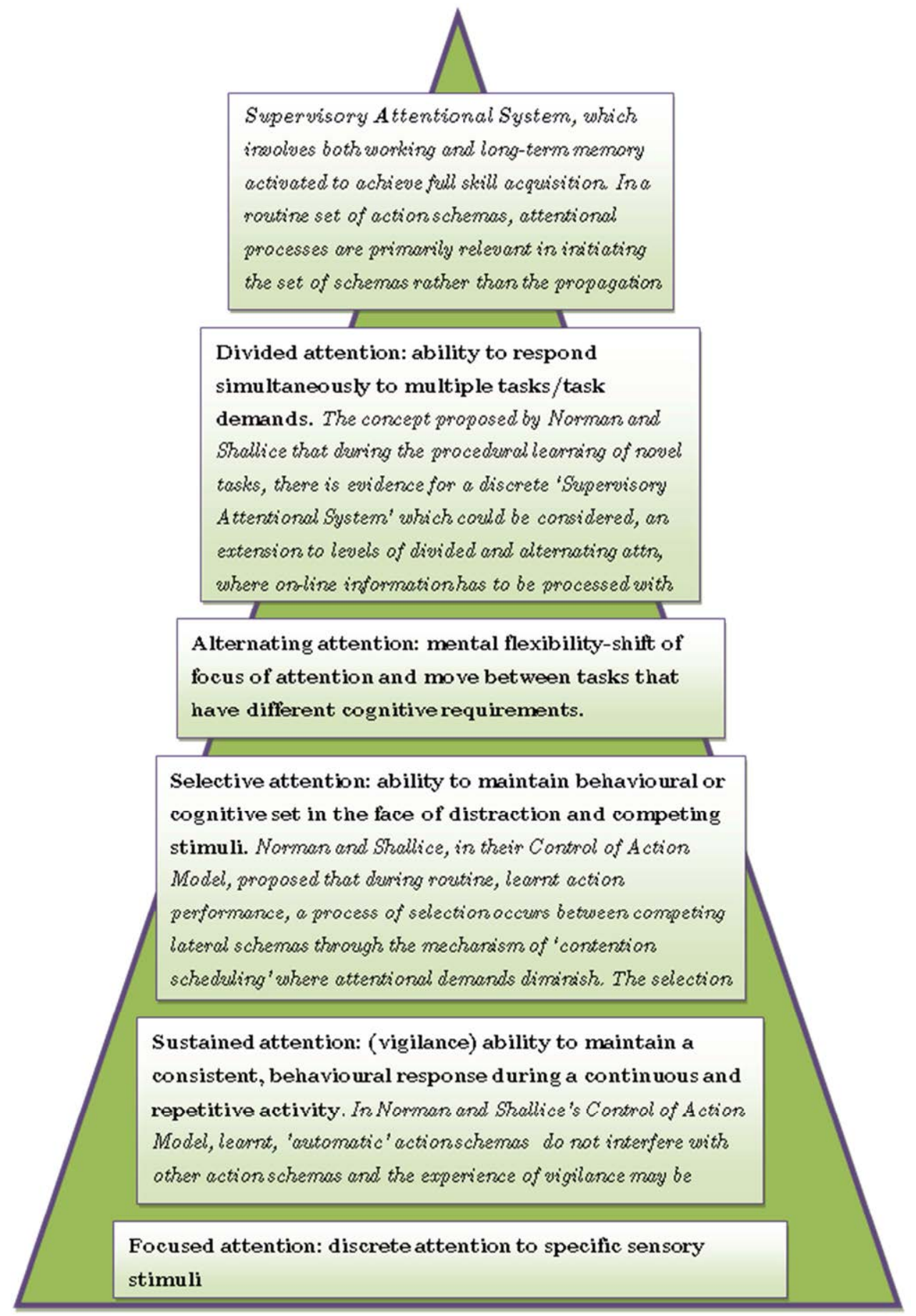

Figure 27. Model for improving attention postulated by M. Solhberg and C. Mateer.

\subsection{Feedback system description}

As exposed in previous chapters, chapter 2 and 3, one of the advantages and principal applications of describing a task model resides in the opportunity to bring contextualised information to the final user. 
The following figure, Figure 28, describes the general structure and the interactions between the modules with the user acting as the actor and the system as the critic.

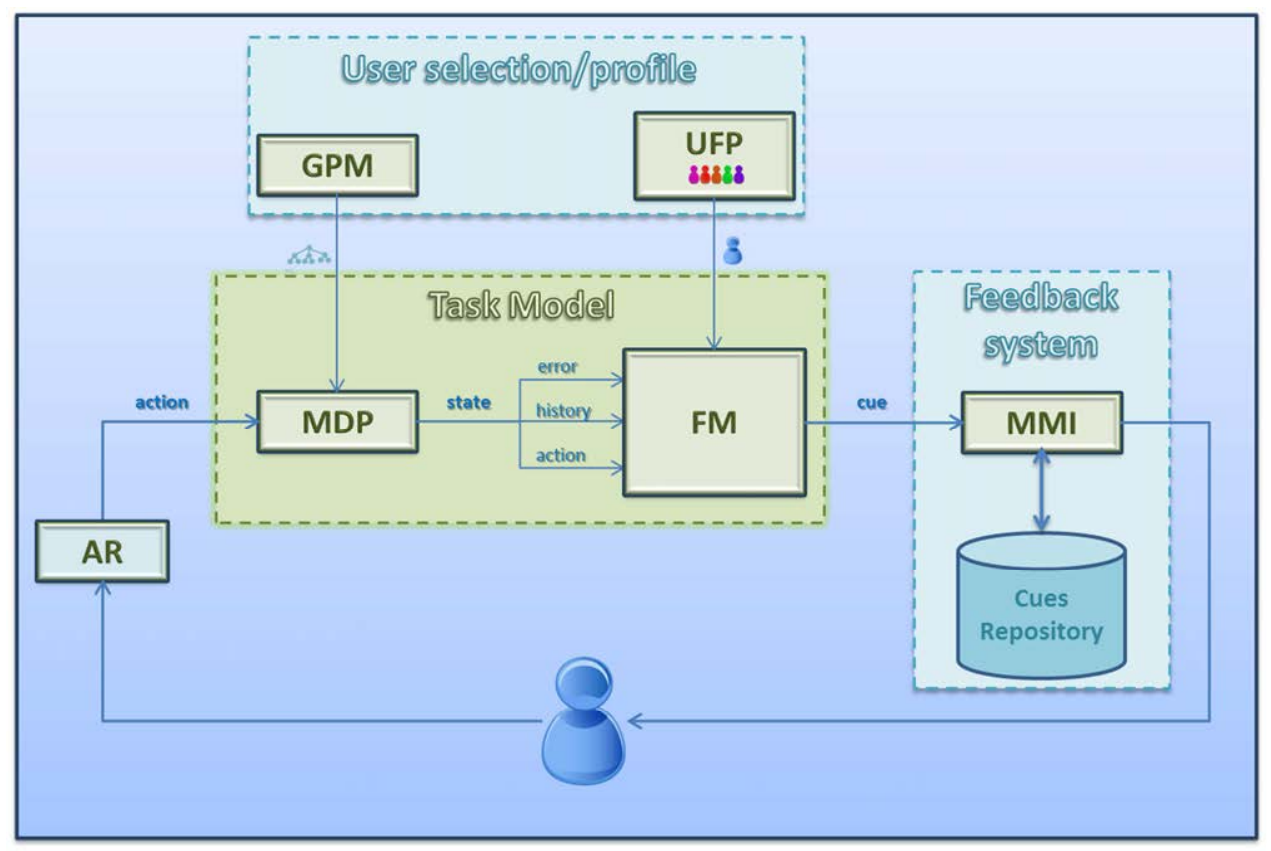

Figure 28. General architecture of the task model-cueing system.

Notice that while the user interacts with a given environment with a finite set of possible actions and errors characterized by a previously task analysis an Action Recognition (AR) module is in charge of detecting the actions made by the patient. There are several strategies to carry out this procedure and the complexity of this matter comprises the topic of a possible thesis. To name some of these strategies, automatic action recognition could be based on Hidden Markov Models (HMM)[170], vision such as [171], with biological inspiration [172], correlation maps[173], analysis of dense trajectories[174] and inference systems from different areas of Artificial Intelligence(e.g. neural networks [175], fuzzy logic [176] and vector support machines[177]). In this dissertation the problematic of action recognition is not treated. On behalf, in chapter 5, a solution for user monitoring has been studied, a deeper review of this matter is provided in [178].

The task model provides the information about the current state which includes: 
- The error committed by the patient

- The history of successful actions performed by the patient

- The next best action suggested by the planning strategy.

- The modality of the information to be displayed to the user.

\subsection{Definition of cues}

The modality of the information and how is displayed to the user is included in the structure defined by the profile module previously described. The cueing structure is defined as a discrete set of cues distributed in time by the amount of times that its' associated error is committed.

According to the modality of the cues, the following modalities has been considered, the modality of this cues has been defined in collaboration with psychologist from UoB and TUM.

- Alarm cues

\section{○ Warning sound}

A typical warning "beep" sound at the frequency of $440 \mathrm{~Hz}$ of adjustable duration (pers. comm. M. Bienkiewicz)

○ Haptics

A vibration of adjustable duration and amplitude is provided. For this purpose a smart watch of the brand Metawatch ${ }^{\mathrm{TM}}$ was used. This watch is based on FreeRTOS and is connected via Bluetooth with the cueing interface through the protocol described in [179]. The use of vibrations it should be not only considered as an alternative warning for the hard of hearing users, it implies and additional source of information supposing a richer communication and enhanced reaction times.

- Visual cues

○ Stills

A static picture represents symbolically an action.

○ Videos

A first person recorded video of the performing of an action.

- Auditory cues

\section{○ Oral messages}


An auditory order, e.g. "Stir the solution".

\section{$\circ$ Ecological sounds}

Based on acoustic ecology from the studies started by R.M. Schafer [180]. It is the emulation of a sound described in an environment and that is perceived and associated with a corresponding activity.

- Written cues

It is the written transcription of an oral message.

\subsection{Cue design}

For assisting the professional in the design of cues the profile module includes a drag and drop application to set the structure of the cues in a simple manner.

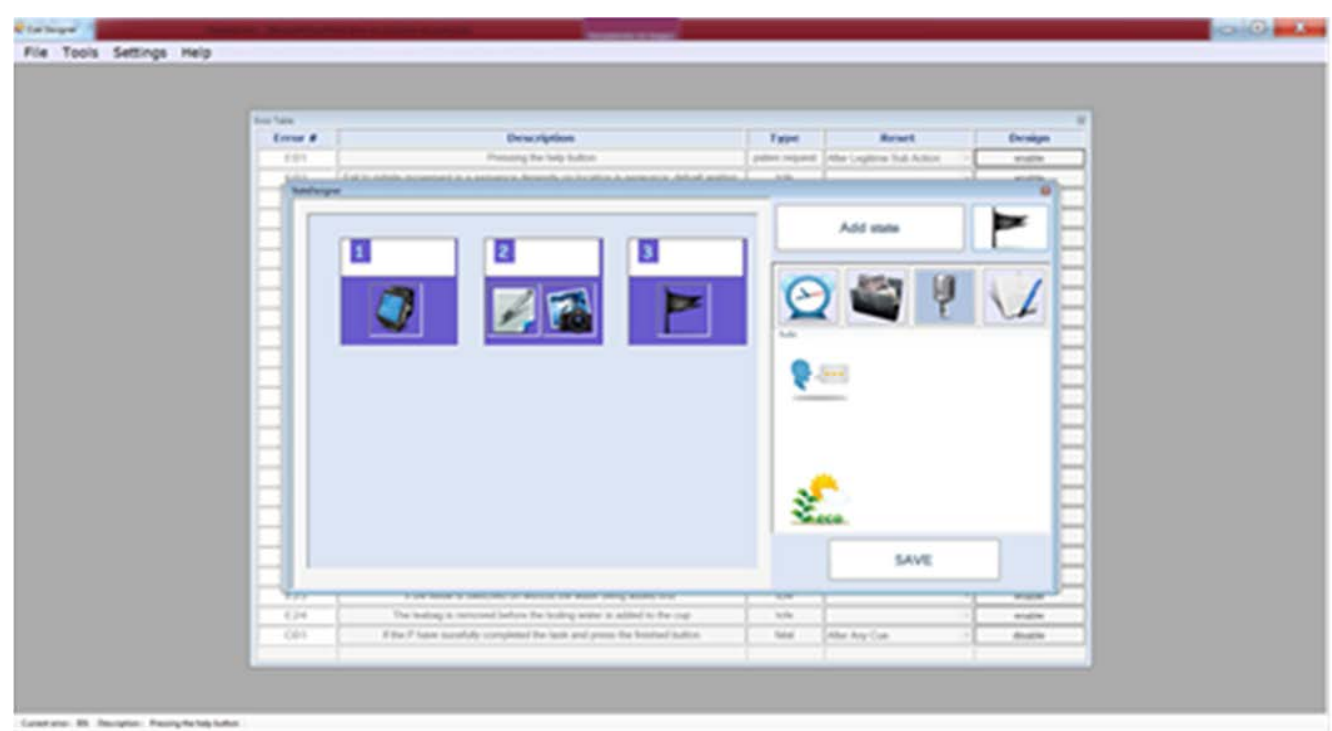

Figure 29. Interface snap shoot of the cue designer.

Each cue is defined as a batch amount of information displayed to the user in a sequence of different stage displayed at a time, see Figure 29. The developed tool allows modifying the structure of each cue individually according to a given set of rules: 
- Two cues of the same modality cannot be include in the same stage, i.e. cannot be reproduced at the same time.

- Visual and written cues must be displayed without distractors, e.g. a lettering animation.

- An alarm is considered a non-informative cue, so, cannot be displayed at the same time than an informative cue.

\subsection{Considerations}

This section described some considerations to take into account for the correct displaying of cues.

\subsubsection{Ipsilateral or contralateral}

Visual cues should consider the dominant hand and display the information from the visual perspective of a left/right handed person. In the special case of post-stroke patients this quality becomes essential because these syndromes are usually combined with hemiplegia.

\subsubsection{Language}

Written and auditory cues are constrained by regional settings, to deal with geographical constrains it is necessary to define each cue to be understood by the user.

\subsubsection{Contextualization}

The information can be show taking into account the previous progress made by the user and displaying visual cues taking into account the updated status of the global action, e.g. if the user is cued to screw a part of an assembly already placed, in the cue, the part must appear in the updated position. To contemplate this classification it must be taken into account that the set of actions is finite and not all the actions conditions the status of each cue. The possible combinatory of $n$ elements (i.e., $n$ is the number of activities to perform a task) in groups of $\mathrm{k}$ elements (where $\mathrm{k}$ evolves from 0 to the depth of the graph $\mathrm{m}$ ) is expressed by the combinatory without repetitions:

$$
\sum_{\forall K}\left(\begin{array}{l}
n \\
k
\end{array}\right)=\sum_{k=0}^{m} \frac{n !}{k !(n-k) !}
$$


The relational description among each error and action can be made by this application to label the contextualized cues according to all possible ordered combinations. Notice that the task model filters the wrong sequences reducing the number of combinations.

\subsubsection{Prospective vs Retrospective}

A cue is considered prospective if it alerts the user of an error who she/he has no committed already, and retrospective if it has been committed.

There is a big discussion in psychology about which kind of cue is more effective and better promotes learning. The work carried out in this thesis only comprises retrospective cueing. The idea underneath rehabilitation is to avoid guiding the user and let her/him proceed by their way. To properly define a prospective system it is required an "early" recognition system able to capture the intention or beginning of execution of each action. Since this AR system was not available the proposed system, Figure 30, has not been implemented. This system considers that the intended actions can be treated as observation in a POMDP based task model.

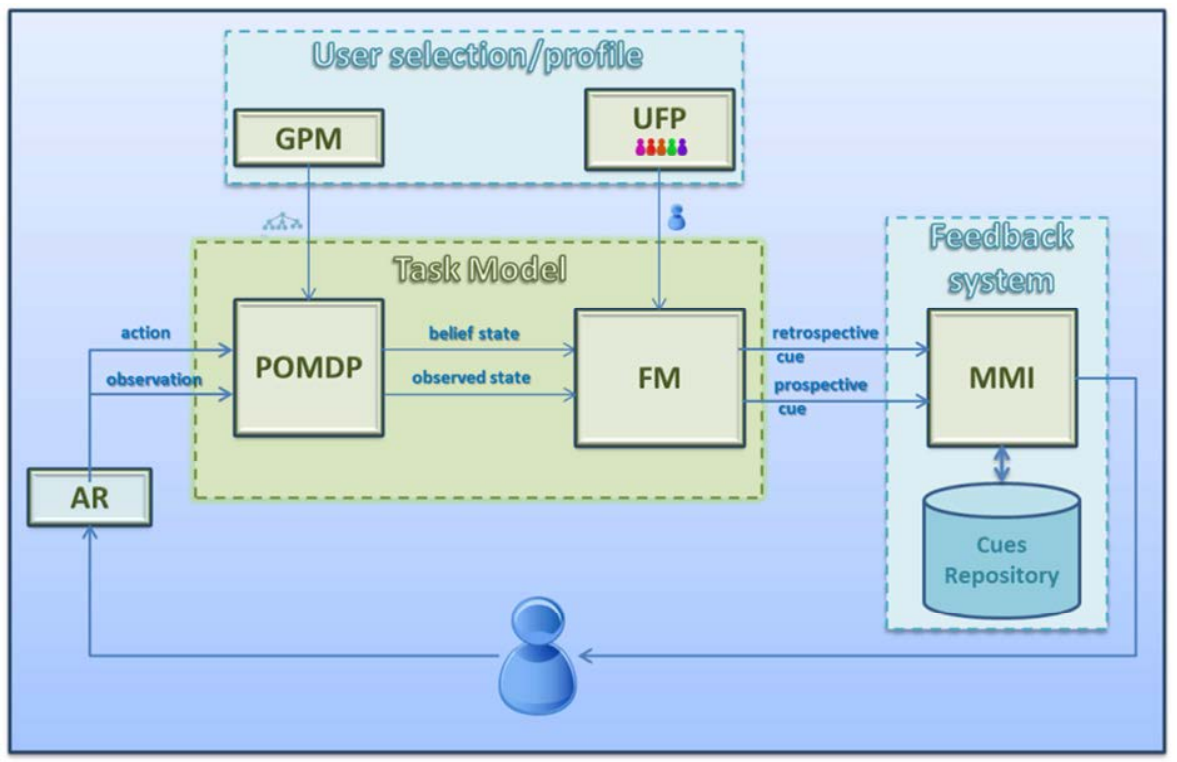

Figure 30. Proposed architecture for prospective cueing. 


\subsection{The multimodal interface}

In this section a definition of the most important factors to take into account at design time of a multimodal interface are presented. From literature could be conclude that multimodal reinforcement is always better than unimodal due to the account of information perceived by the user but it depends on the way of showing this information, so, user can perceive and interpret the stimuli correctly. For example, assuming perfect visual and auditory conditions for perceiving the stimuli, if a system emits a phonetic /ba/ while showing an image of a lip movement simulating a /ga/, the user will perceive a false stimuli /da/ [162]. This is an example of Categorical Perception; a matter widely studied by cognitive psychology.

It is necessary to describe the requirements of functionalities of a multimodal interface applied to cognitive rehabilitation. It must be developed an interface which supports all the communication channels: described in section 4.3, the restriction from section 4.4 and the considerations from section 4.5.

Other functionalities that the interface must fulfil are: i) interactivity with the user to correct on real time the errors and prevent potential hazardous actions, ii) the user can interact with the system gathering information in case that assistance is required, iii) the system must confirm the willingness of the patient to start and finish a session, if the user considers that the task is concluded and iv) due to ethical and safety reasons the system must allow the user to abort the session at any time without registering any data from the session. This final requirement is the most important and it is a topic largely discussed in the field of AI and, hence, in Task Modelling. The methodology of Reinforcement Learning applied in this thesis has been demonstrated to be inherently safely interruptible in the recent and famous publication from L. Orseau and M.S. Armstrong [181] where the safety switch is mathematically demonstrated for AI agents.

It is also desirable that the layout of the display is dynamic and re-sized depending on the cues to display, e.g. in Figure 31 the display is re-sized to show the cues optimising the display area, if the video were not required the text would appear in full screen and vice versa, 
Additionally the system should include different layouts to inform the user about the actions already done and the general goal. The progress of the task is included in the upper panel where each action made by the patient is represented by a characteristic still. The goal is represented in the upper right corner by a representative still of the global task.

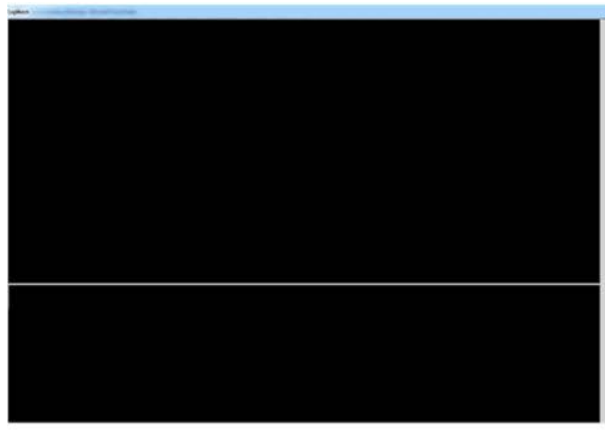

Without Cue Progress \& Cue Goal

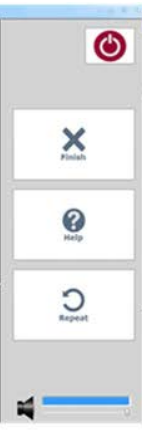

(1)
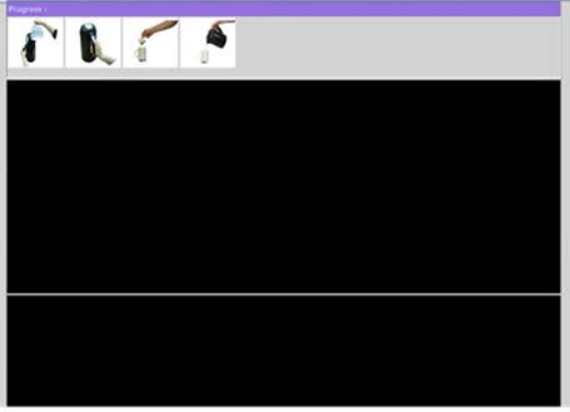

Only with Cue Progress

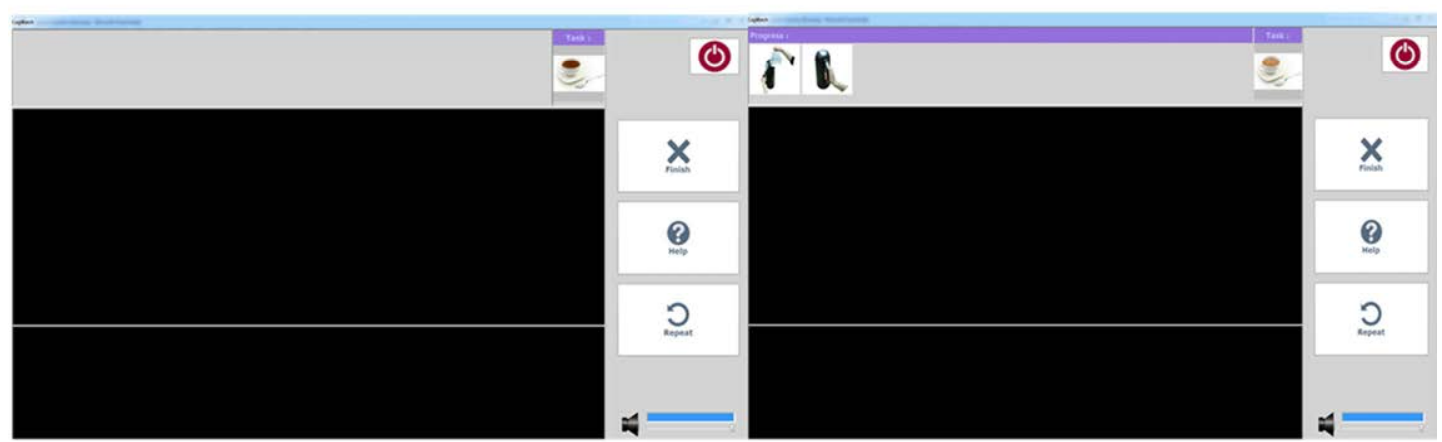

Only with Cue Goal

(1)

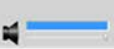

With Cue Progress \& Cue Goal

Figure 31. Interface layouts for additional assitive information, cue progress and cue goal.

N.B. that the requirements and functionalities of the multimodal interface meet the definition from an agent described in section 1.2.

\subsection{Experiment for learning interfaces validation}

As presented previously in the beginning of the article, one of the main objectives of this contribution is to improve the current techniques of cognitive rehabilitation by implementing a platform that use multimodal interfaces which interact with the user at the same time the clinician responsible for the rehabilitation session is supervising the task, and communicating with patient if necessary, remotely from another different room or even from the hospital if the patient is executing the task at home. 
This means that there won't be need of moving to the hospital or rehabilitation centre to carry out the session and even more it will discharge therapists from their workload of helping personally the patient to execute the activity.

In order to demonstrate the applicability and validate the use of the multimodal interfaces, an experiment focused on the execution of simple tasks never done previously by the user is considered with and without the help of the interface to measure the improvement in the execution and the cognitive model during the sessions.

For that purpose, 15 healthy participants have been chosen in order to carry out the activities. The tasks selected have been to put in order a sliding puzzle and to play the song "Jingle Bells" using a virtual piano. The participants have poor formation in music so to learn how to play this song is a challenge for them. The following figure, Figure 32, shows a graphical representation of the methodology implemented:

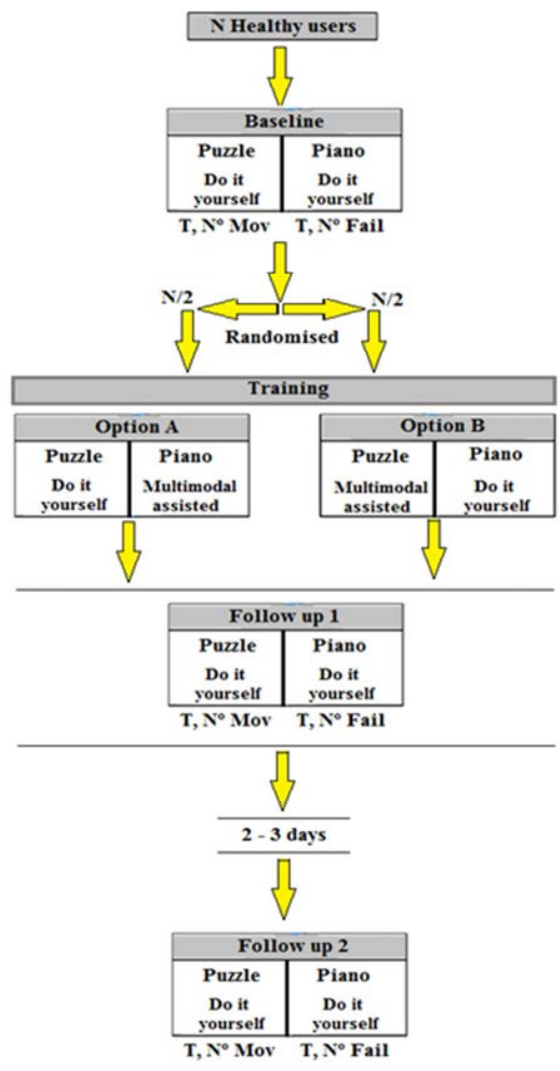

Figure 32. Experiment methodology for learning how to put in order a numbers puzzle and play a song with a piano. 
As presented in the figure, two different options were created to divide the initial number of participants into the half in each option taking advantage of the dichotomous nature of the experiment.

Initially, all the participants were undergone to execute both tasks without help in order to fix a Baseline (BL). Then, half of the participants were randomly assigned to option $\mathrm{A}$ while the other half were assigned to option B. These options mainly differ on the training phase. In option $A$, the participants had to put in order the sliding puzzle, Figure 33, without any help and after that try to play the song with the help of the multimodal interface and cues. Meanwhile, participants who were assigned with option B had to perform the same tasks but in this case, the help of the interface was focused on the order of the puzzle only. The two options correspond to a phase of training in order to better evaluate the execution of the task taking into account before and after being familiarised with them. It is important to highlight that no data registration is done during the training.

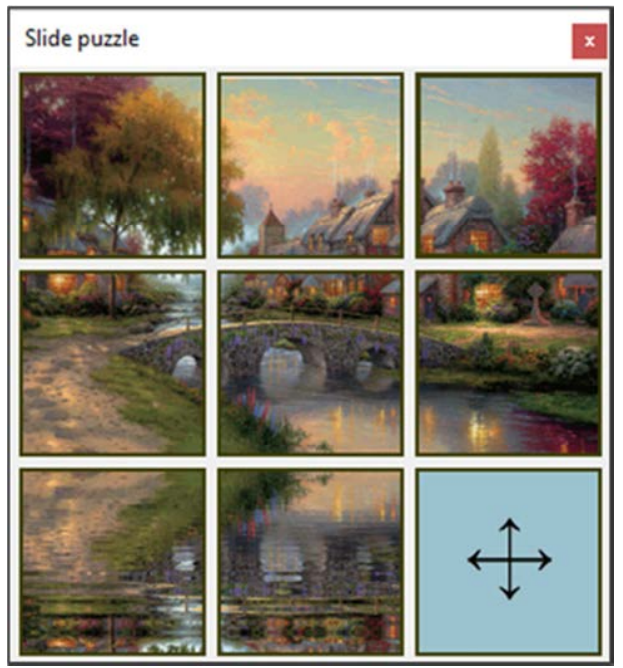

Figure 33. Correct disposition of the puzzle.

After finishing the performance implemented in the options, the users had to execute both tasks again without any help, Follow up 1(FU1), like in BL in order to measure the level of improvement and differences between those people who were aided by the multimodal interface and those without help. Moreover, after 2 or 3 days, all the users must perform the two tasks again without any help, Follow up 2(FU2), to measure also the capacity of training 
and their memory making difference between tasks guided with multimodal cues and without them.

In general, the main parameters measured to analyse the improvement in the execution of the task and the level of learning were:

- Execution time of both tasks.

- Number of movements needed to put in order to puzzle.

- Number of errors while playing the whole song.

Regarding the performance of the song without any help from multimodal interfaces, the users only had an audio to try to repeat the same melody just looking for the sound of the notes along the keyboard, sometimes complemented with a video of a piano also playing the song.

It is important to mention that in order to make it easy for the participants, the specific keys from the keyboard that correspond to each note that compose the song were pointed out just to limit the part of the piano's keyboard to that scale used in the song, see Figure 34:

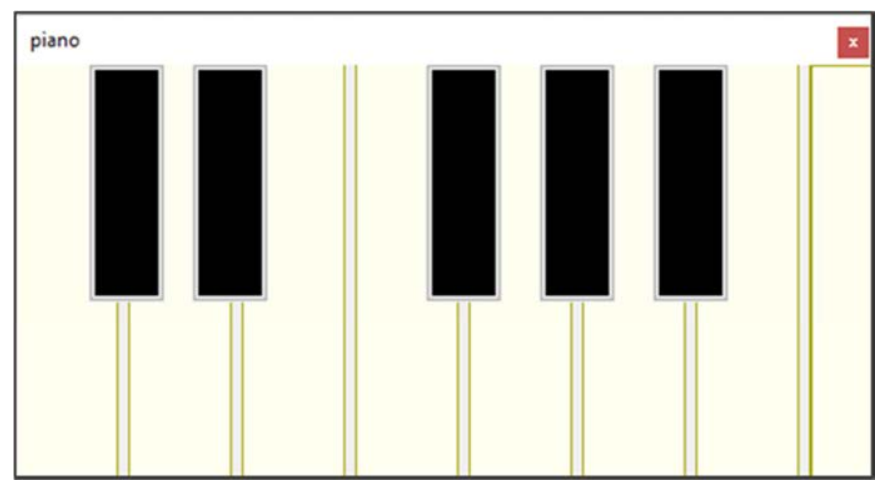

Figure 34. Piano's keys used to play the song Jingle Bells in Do.

The errors considered to automatically prompt a multimodal cue are related mainly with the following topics:

- The user has doubts in executing the following action (movement of the puzzle or key selection from the piano). A time counter exceeds a specific limit without any action from the user.

- The user fails in performing a step (playing the wrong note or making a movement of the wrong piece). 
Regarding the cues to use when the tasks are aided by the multimodal interface, these are associated with:

- Still images. An image shows the puzzle piece to move, Figure 35, or the following note to play, Figure 36.

- Video. An animation indicates the sequence of several notes to interpret the compass in which the user is blocked, or the real movement of the correct piece in the case of the puzzle.

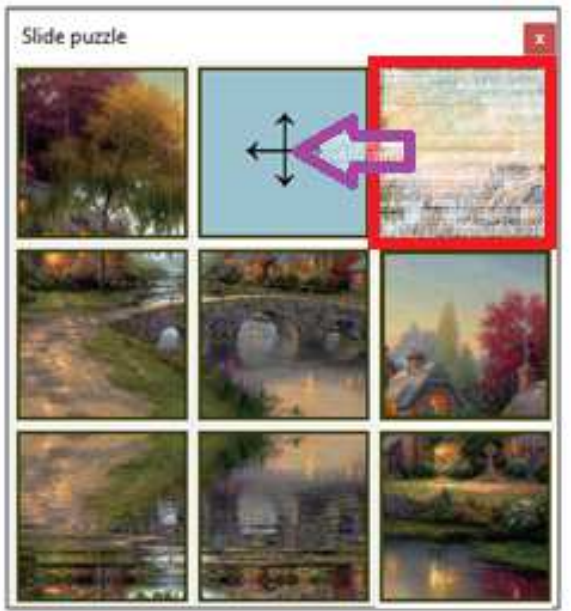

Figure 35. A still image indicates the correct piece to move.

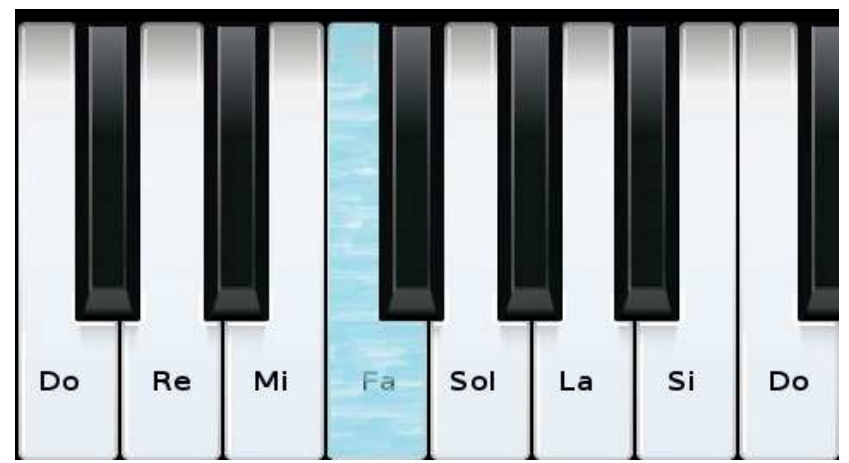

Figure 36. Still image indicating which note to play.

Regarding the performance of the Christmas song, each cue is also accompanied by the sound of the specific note, in the first case, or by the short melody, in the second one. The animation always displays all the steps done before, i.e. it accumulates the musical compasses played from the beginning and the different correct movements done by the participant, so when the user receives a video, he/she always must repeat the sequences from the beginning to the point where the error was committed. The cues are 
prompted automatically when an error is committed by the user thanks to the Task Model previously mentioned which is in charge of error detection.

For analysing results it has been chosen non-parametric test to answer the different questions from the study:

- Can the interface assist to learn?

- The method provided enhances the learning curve from classical methods?

- The performance time decreases between methods?

The advantage from non-parametric methods is that it is not necessary to make any assumptions about the type of distribution of the sample. In the case of not been possible to assume a normal distribution non-parametric results are stronger than parametric ones when the Central Limit Theorem cannot be met. That is the reason because some statistics prefer nonparametric methods[182].

\subsubsection{Analysis of results from the experiment}

One of the uncertainties exposed in the previous sections was that it is not always clear which kind of assumptions over the population are correct or not. The data from the 15 participants were analysed with $\mathrm{R}$ 3.2.3 [183]. The initial sample was contrasted to test for normality by using the Lilliefors test, for example in the case of FU1 for piano test null hypothesis was rejected at significance level of $5 \%$ with $\mathrm{p} \cong 8.334 \cdot 10^{-6}$ computed with Monte Carlo simulation and in the case of FU1 for the puzzle test the hypothesis was also rejected with $\mathrm{p}=5.4167 \cdot 10^{-4}$. The first conclusion from this test is that the samples collected cannot be decided in terms of a mean and a standard deviation, instead, data are better explained in terms of the median and the range.

In the following figures, Figure 37 and Figure 38, the results from the data collected are summarized. In Figure 37, the learning curves for piano test are showed represented by the median of both the number of errors and the time required for the task during the three stages previously described. In the left side the decrease in the number of errors seems to be sharper in the case of the assisted training (labeled ' $\mathrm{CW}$ training'), but 
must be also noticed that the representative number of errors during BL is higher than the value for both types of training together ('Global trend'). Task execution duration, on the other hand, shows more clearly a faster execution after the assisted training.

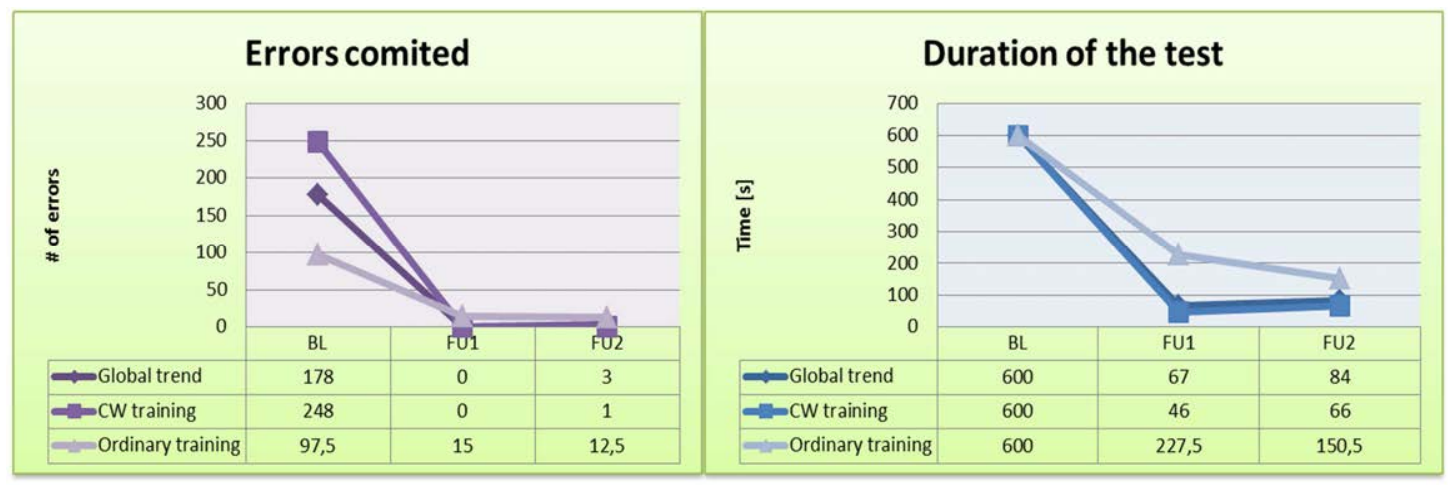

Figure 37. Results for piano test.

In the case of the sliding puzzle test, similar results are displayed in Figure 38. Opposite to the case of the piano test, the learned strategy from the training phase was learned and more steady retained as remarked by the plateaus on the curve.

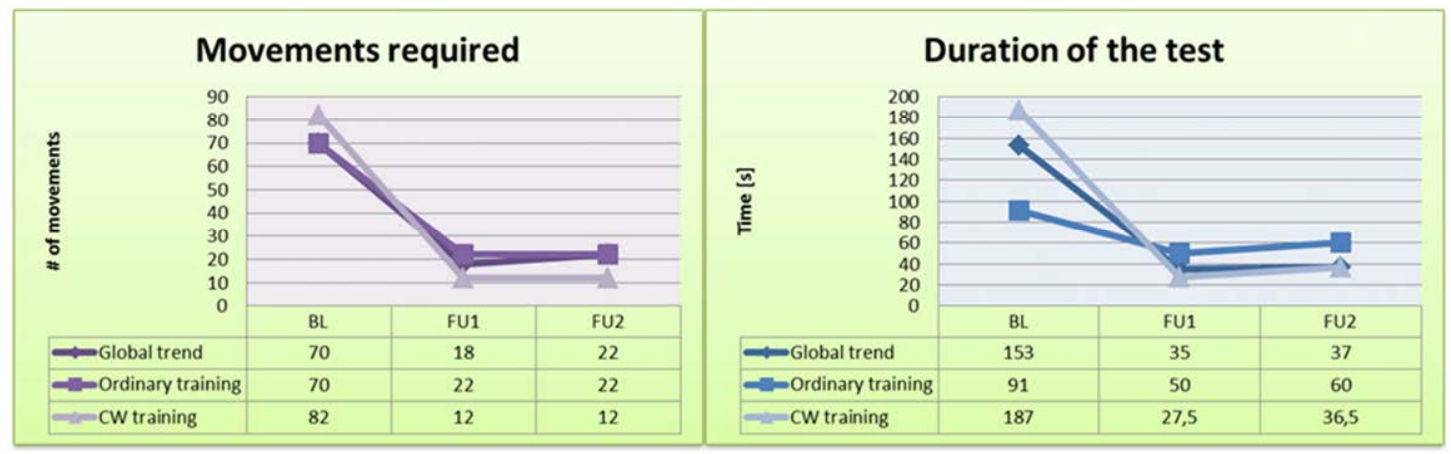

Figure 38. Results for sliding puzzle test.

The results displayed could explain a better performance after assisted training but it is a requirement to execute a contrast test to check the veracity of statements exposed. As previously explained, the suitability of non-parametric test is, by far, demonstrated. Different test could be used. Data can be transformed to contingency tables in case of nominal or categorical data if the object of the study is to answer a single question. In that case Cochran test combined with McNemar test are a good strategy to deal with studies where there is a need to add some information such as how 
the learning varies among categorical groups (i.e. clustered by gender or age ranges)[184]. A similar test can be performed applied to ordinal data. Friedman test was selected for the study, which is the non-parametric alternative to the one way ANOVA test for multiple measurements. The test is suitable for ordinal paired data and it has been performed for both the piano and the sliding puzzle tasks. In order to deal with the ties it has been used the corrected formula for the statistic F.

$F=\frac{12 \sum_{i=1}^{k} R_{i}^{2}-3 N^{2} k(k-1)^{2}}{N k\left(k^{2}-1\right)-\sum t\left(t^{2}-1\right)}$

Where: $\mathrm{R}$ is the rank number for each group, $\mathrm{N}$ is the number of participants, $\mathrm{k}$ is the number of groups and the number of ties. This statics follows a $\chi 2$ distribution with k-1 degrees of freedom.

The $\mathrm{p}$ value obtained for the piano test was $\mathrm{p}=1.2513 \cdot 10^{-5}$ and the value obtained for the puzzle results was $\mathrm{p}=1.3265 \cdot 10^{-5}$ both below 0.05 , what means that there are significance difference between both types of training complemented with a post-hoc analysis using a Tukey test which reveals that the most significant different are given between the BL and the FU1 stages and the improvement is higher in the puzzle task than in the piano task.

\subsubsection{Conclusions from the experiment}

The analysis of results has concluded that a significant difference can be established between the assisted training and the ordinary training. Some interesting remarks found are that in some cases the number of errors during the piano test where reduced between the FU1 and the FU2 what could explain that the participant has not learned correctly the task during the training stage and completed his training during FU2. The fact that the assisted training obligates the participant to perform the entire musical piece correctly from the beginning made the participants memorized the task as a whole. The improvement on the assisted puzzle test with 6 out of 7 participants performing the FU2 in the optimum number of moves (twelve) reveals that the strategy was easy to be learnt and the puzzle task was easier that the piano task. Analog tests could be done considering the task durations. 
The test presented in the experiment can be applied in different situations and complemented with ranking tests to consider the row effects as demonstrated in the post-hoc analysis. This is a simple methodology for measuring the efficacy of an assistive interface without the necessity of large datasets. It is especially helpful when the study must deal with categorical data or ranges. It can also be applied for usability surveys where the data is collected in ordinal data such as the Likert scale.

\subsection{Lessons about multimodal interfaces}

In this chapter, it was described how the use of multimodal interfaces can handle several communication channels and the benefits from this strategy. To transmit information with effectiveness the cues provided must be adapted by taking advantage from a user profile and take into account the limitations and preferences from this kind of structured information. Furthermore, it is recommended to contrast the information to display with an internal model of the task. Benefits from this strategy let the system provide contextualized feedback. Thereafter contrasting with the model, personalized feedback becomes adaptive and therefore a better learning tool. This is the ultimate objective of rehabilitation. The use of task modelling for supporting contextualized guidance is, hence, a recommended practice. 



\section{CHAPTER 5}

\section{Monitoring THE USER ACtions AND PERFORMANCE}

The chapter is focused on the factors involved in monitoring the user. As reported in the study from the literature, monitoring is considered another application field of task modelling; in order to complete the review of the topics covered by task modelling it has been presented an approach for user monitoring. This chapter aims to justify the use of non-invasive monitoring systems to be included in the recognition phase of the task model described in chapter 3. Additionally, considering the application purpose of the system, it has been studied additional parameters to characterize the physiological state of the user in the context of cognitive rehabilitation.

In the first part of this chapter, a research carried out in collaboration with the TUM and focused on the use of a NUI sensor as an adequate sensor for kinematics analysis to be applied in cognitive rehabilitation of AADS patients is presented.

The main objective is to prove that this low costs devices are suitable, accurate and effective enough to be used in the analysis and movement tracking of ADL performance taking into account its low cost and easy implementation. For that purpose, information regarding data collection and analysis of kinematic data obtained from the NUI sensor during the 
performance of an activity of daily living (i.e., making a cup of tea) is provided.

After that, a reaction time study has been carried out. The motivation of this study is twofold: fist it is required to validate a device for measuring reaction times from the user to visual and auditory stimuli and second, it is compared the reaction times of healthy volunteers to understand which stimuli are better to provide alarms to the user.

Finally, the suitability of monitoring physiological signals is reviewed and the use of a medical device for monitoring Blood pressure is proposed.

\subsection{Experiment for Natural User Interface sensor validation}

The experiment is carried out by two people: a healthy person, in order to evaluate the system more technical and a patient who suffer apraxia, to prove the performance considering a real case of use. Moreover, Kinect ${ }^{\mathrm{TM}}$ data is then compared to the kinematic data collected at a higher sampling rate from an ultrasonic motion capture system (Zebris). The results are used to make comparison between both systems, and show the suitability of Kinect $^{\mathrm{TM}}$ for investigating kinematic patterns of movement in ADL tasks.

The set-up used for the experiments is made up of the Kinect ${ }^{\mathrm{TM}}$, which is the most relevant component, and a central processor, which is responsible for: (1) receiving image and position data from the Kinect ${ }^{\mathrm{TM}}$, (2) computing all the algorithms for analysis, and (3) providing user interface to interact with the Kinect ${ }^{\mathrm{TM}}$.

It is important to mention that in order to ensure flexibility and to make the system more ergonomic and simple, the central processor and the monitor are embedded in an all-in-one computer, which provides the CPU and screen in only one device.

Finally, several objects are selected from the corresponding ADL task in order to simulate the preparation of tea. These are a kettle, a coffee cup, and a tea bag which are presented later in Figure 41. 
According to the methodology to follow during the execution of the experiment, Figure 39 shows a block diagram representing the whole process designed since the data acquisition from the Kinect ${ }^{\mathrm{TM}}$ to the plot and analysis of the signal:

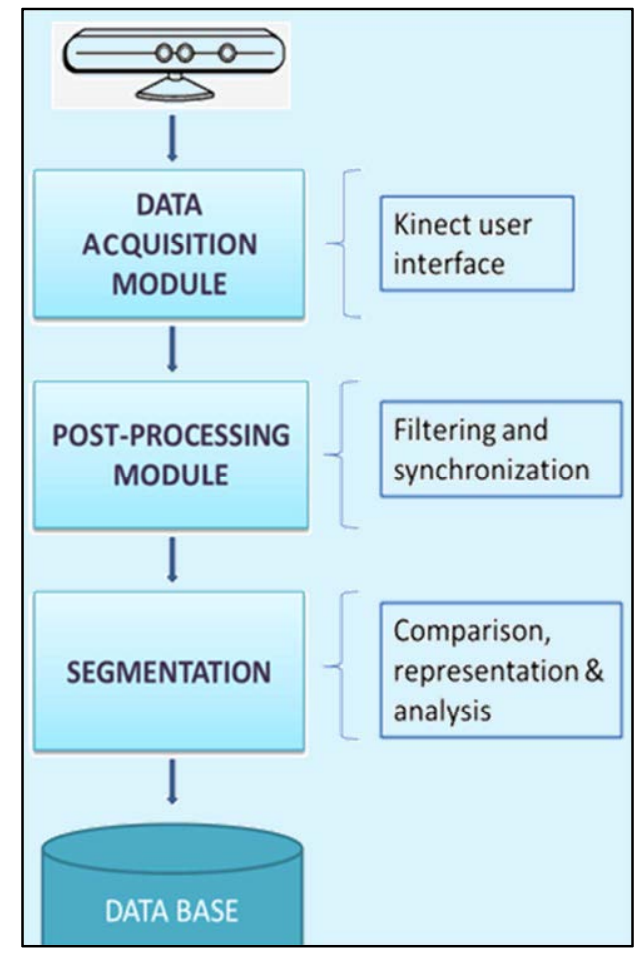

Figure 39. The figure shows the modular methodology used with the Kinect ${ }^{\mathrm{TM}}$ for data analysis. First, the data is acquired so as to launch the camera sensor using Kinect SDK@ functions and the interface developed, second, the data is filtered and synchronized, then the segmentation in epochs is carried out to represent and analyse the data. Finally, the information is stored.

First, the recording interface lets the user choose between different options related to the mode in which the Kinect ${ }^{\mathrm{TM}}$ records the information from the cameras. One of the advantages of selecting the mode is that the user can customize the video image. Instead of recording the whole body, the Kinect ${ }^{\mathrm{TM}}$ can record only the data from the upper half of the body. This feature is pertinent to obtain information strictly from hand movements during ADL performance.

Besides the different images from the whole scenario, another functionality provided by the interface is the possibility of visualizing in real time the $\mathrm{X}$, $\mathrm{Y}, \mathrm{Z}$ positions of the hand (exactly, of a fixed point in the wrist). The interface, which has been programmed using $\mathrm{C}++$ in Microsoft Visual 
Studio, has implemented a final option of saving all the position data in a file with a specific format selected by the user.

Secondly, once all the data is saved, all the data is post-processed by loading the files saved before using Matlab in order to plot the signals and study if any filtering is needed. As shown in the following section, filtering has been focused on Butterworth. A Butterworth filter is applied to obtain maximum flatness in the band pass. For this article, it is applied in its digital version that is an IIR filter. The Butterworth filter provides the best Taylor Series approximation to the ideal low-pass filter response at analog frequencies $\mathrm{fr}=$ 0 and $\mathrm{fr}=\infty$. In this case, no stability problems are found in the domain of the input data.

After filtering the signal, Kinect ${ }^{\mathrm{TM}}$ data is compared with the data obtained from Zebris, and used to determine whether the Kinect ${ }^{\mathrm{TM}}$ can recognize handmade movements, and if it is a suitable motion capture system for cognitive rehabilitation.

For comparison, an ultrasonic three-dimensional motion capture system was used as reference system. The Zebris [[185], [186] system features three sonic emitters, which send out packets of ultrasound, and receivers that can be placed on relevant body segments.

\subsection{Experiments and Results}

\subsubsection{Data Collection}

In order to test the suitability of the Kinect ${ }^{\mathrm{TM}}$ device as a motion capture system we collected data from a neurologically healthy 32 year-old female (control participant), and a 47 year-old female with apraxia (apraxic participant). Both participants were right handed. The apraxic participant had left-brain damage which resulted in hemiparesis of the dominant right hand. As such the apraxia patient had the use of the non-dominant left hand only.

For the decomposition of the task it has been done a HTA, as described in chapter 3. Some remarks in this approach is not simply the hierarchical decomposition but also the definition of 'plans'. As in the psychological studies of R. Cooper and T. Shallice [187], hierarchy is typically described 
in terms of decomposition of a "goal" into "subgoals", moving from a highlevel objective to lower-level tasks. In this case and based on HTA, the whole task tree designed for the project and experiments regarding the task focused on tea preparation is shown in the next figure, Figure 40:

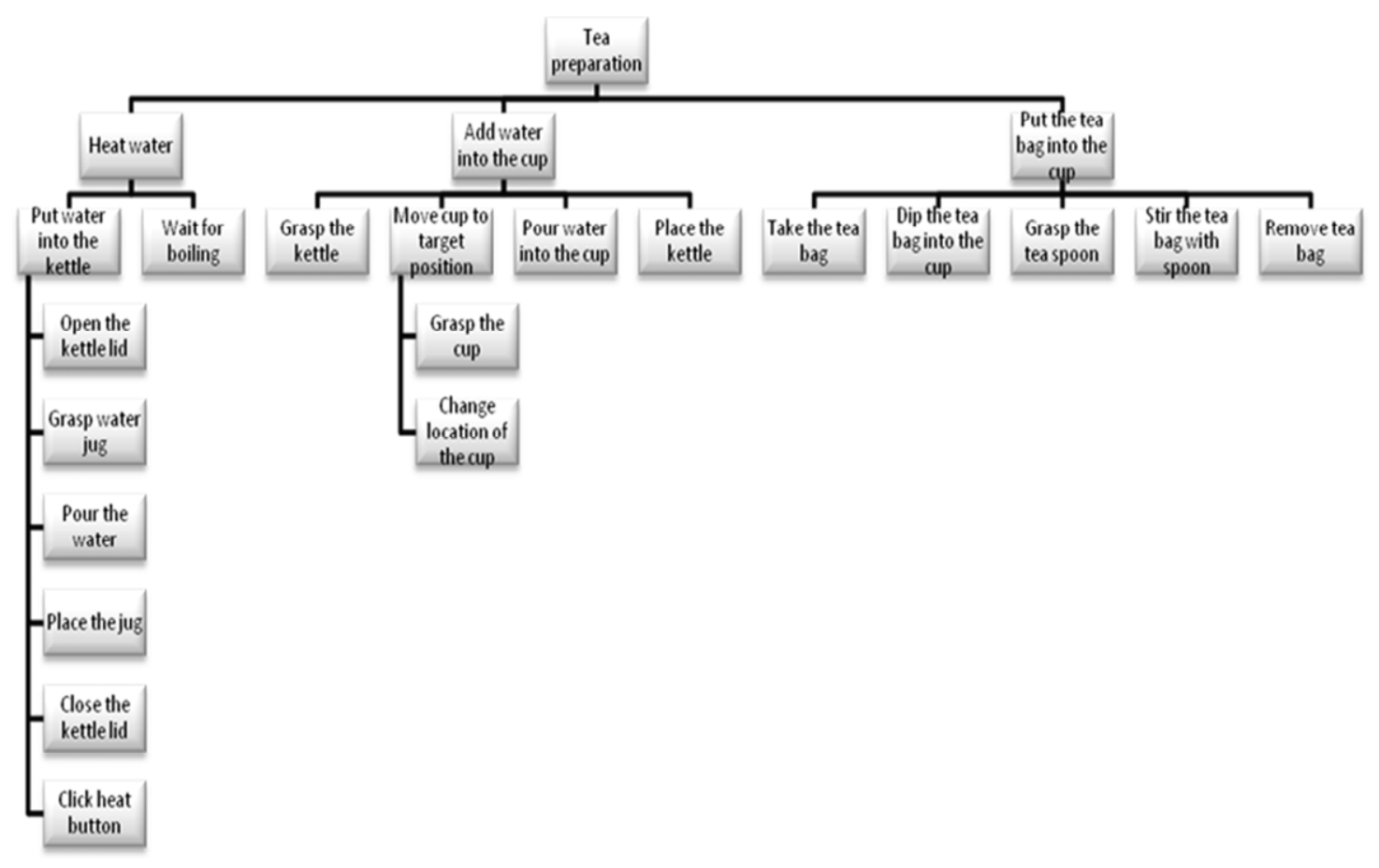

Figure 40. HTA for tea making.

However, in order to simplify the execution and make the experiment faster for the article, the task to be executed is reduced by following these instructions, which specify a particular sequence in which the task should be performed:

※ Reach and grasp the cup.

※ Transport the cup to target position.

※ Reach and grasp the tea bag.

* Place the tea bag into the cup.

※ Reach and grasp the kettle.

※ Transport the kettle to position over cup.

* Pour the water from the kettle into the cup.

※ Place the kettle back on table.

※ Reach the tea bag.

* Stir the tea bag in the cup. 
Given that the apraxic participant was hemiparetic, the control participant was also required to use only the left hand to perform the task. The instructions emphasized that the task must be performed at a normal pace. The participants performed a total of 20 tea-making trials. The entire session took 30 minutes. The objects used in the experiment were arranged as shown in Figure 41:

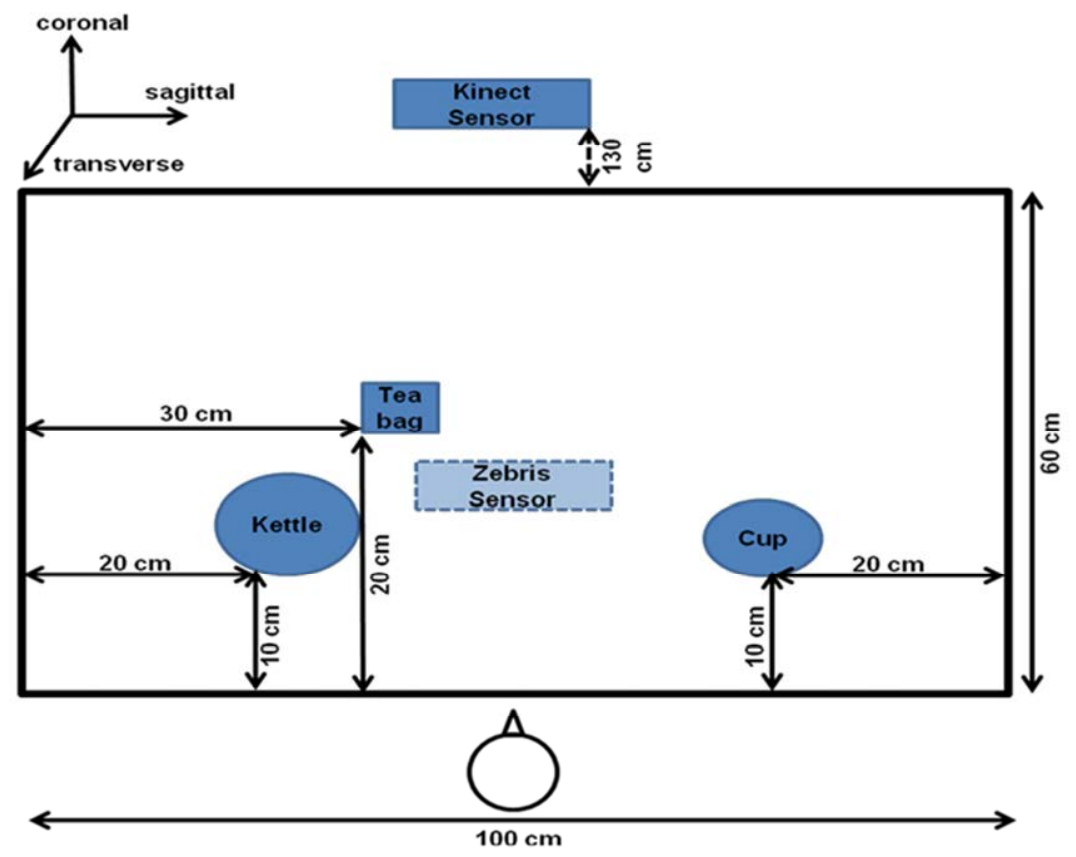

Figure 41. This figure shows the object layout for the experiment. The objects involved. A cup of tea, a kettle, a tea bag; the sensor KinectTM and the Zebris system. The general Cartesian reference system is also represented.

The optimal distance to locate the Kinect ${ }^{\mathrm{TM}}$ sensor in front of the participants was $1.3 \mathrm{~m}$. It was arranged so as to coincide with the participants' body midline. By default, the way the Kinect ${ }^{\mathrm{TM}}$ detects and obtains data from the body of the user is divided into different steps: the first step is focused on the segmentation of the depth data per-pixel, body-part classification. In the second step, the system hypothesizes the body joints by finding global centroid of probability mass (local modes of density) through mean shift. In the last step, the hypothesized joints are mapped to the skeletal joints, and then fit to a skeleton by considering both temporal continuity and prior knowledge. Thus, the body is represented as a stick figure consisting of line segments linked by joints (e.g., head, neck, shoulders, and arms), with each joint represented by its 3D coordinates. 
Kinematic data was collected by the Kinect $^{\mathrm{TM}}$ (30 Hz sampling rate) and the Zebris systems (120 Hz sampling rate). The Zebris system was used as reference system, and consisted of a sensor placed $1 \mathrm{~m}$ above the table, and a marker (1 cm diameter) which was fixed to the dorsum just proximal to the space between $1^{\text {st }}$ and $2^{\text {nd }}$ metacarpophalangeal joint of the left hand. The Zebris data was processed using WinData software 2.19.14 [188].

\subsubsection{Data Analysis}

In the first step of data analysis, the kinematic data was loaded in a custom written MatLab program (The MathWorks®, Version R2010a), and the 3D coordinates were low-pass filtered at a $5 \mathrm{~Hz}$ cut-off, using a first order Butterworth filter. The data was also filtered using an infinite response filter, which was selected because it reduced the amount of delay in the signal. It is known to be more computational efficiency than a finite response filter. This low-pass filter adequately smoothed the signal, rejected movement artefacts and high frequency phenomena (e.g., aliasing), and eliminated power line harmonic interferences Figure 42 shows the signal before (blue signal) and after (red signal) filtering Kinect ${ }^{\mathrm{TM}}$ data:

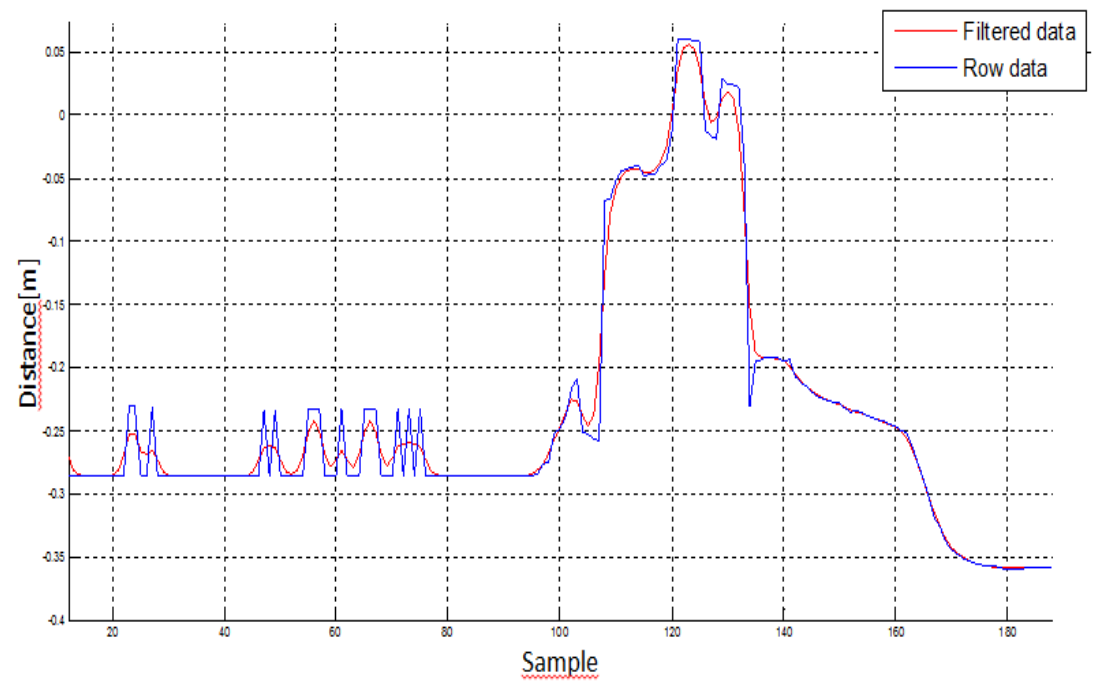

Figure 42. Result of filtering Kinect ${ }^{\mathrm{TM}}$ data with a Butterworth low pass filter.

In the next step, the Zebris data was then down-sampled to $30 \mathrm{~Hz}$ and then normalized by computing standardized z-scores using the mean and standard deviation of the vector for each axes (transverse, sagittal, coronal). This procedure allowed the comparison of the obtained data from the two motion 
capture systems to be done directly. The differences between the Kinect ${ }^{\mathrm{TM}}$ and Zebris data were quantified utilizing Mean Square Error (MSE) and cross-correlation measures separately for each axis. MSE was computed using the Matlab mean squared normalized error performance (mse) function, which measures the expected squared distance between an estimator (Zebris) and the observations (Kinect ${ }^{\mathrm{TM}}$ ). A MSE of 0 means the estimator (Zebris) predicts observations (Kinect ${ }^{\mathrm{TM}}$ ) with perfect precision. Larger MSE values indicate that the observation values (Kinect ${ }^{\mathrm{TM}}$ ) differed from the estimator (Zebris).

Cross-correlations were computed by using the Matlab corrcoef function, and that function was used for estimating the dependence of the values obtained from the two motion capture systems. The correlation coefficient ranges from -1 to 1 . A value of 1 implies that a linear equation describes the relationship between the Zebris and Kinect ${ }^{\mathrm{TM}}$ perfectly. Values around 0 imply that there is no linear correlation between the variables. Negative values indicate an inverse relationship between the variables (i.e., the values of one of the variables increase, the values of the second variable decrease).

In order to ascertain whether the comparison of obtained data from the Kinect $^{\mathrm{TM}}$ and Zebris systems differed between the control participant and the apraxic participant, we conducted a 2 (individual: control participant, apraxic participant) $\times 3$ (axes: transverse, sagittal, coronal) for each measure (MSE, cross-correlation) separately. The $\alpha$ level was set at 0.05 and as such $p$ values less than 0.05 indicate statistical differences.

\subsubsection{Results from the experiment}

Hand position for each dimension and correlation maps between axes for the control and apraxic participants are displayed in Figure 43. It was observed that the Kinect ${ }^{\mathrm{TM}}$ (blue signal) was able to adequate track 3D hand positions, and was similar to that collected by a Zebris marker-based ultrasonic motion capture system (green signal). 


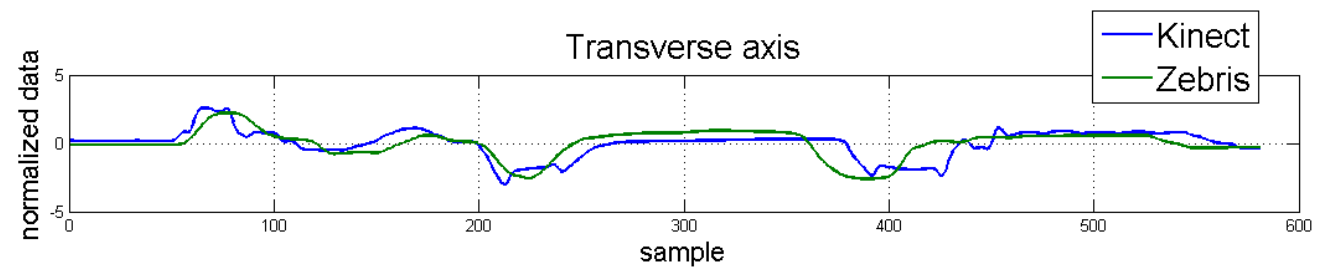

Sagittal axis
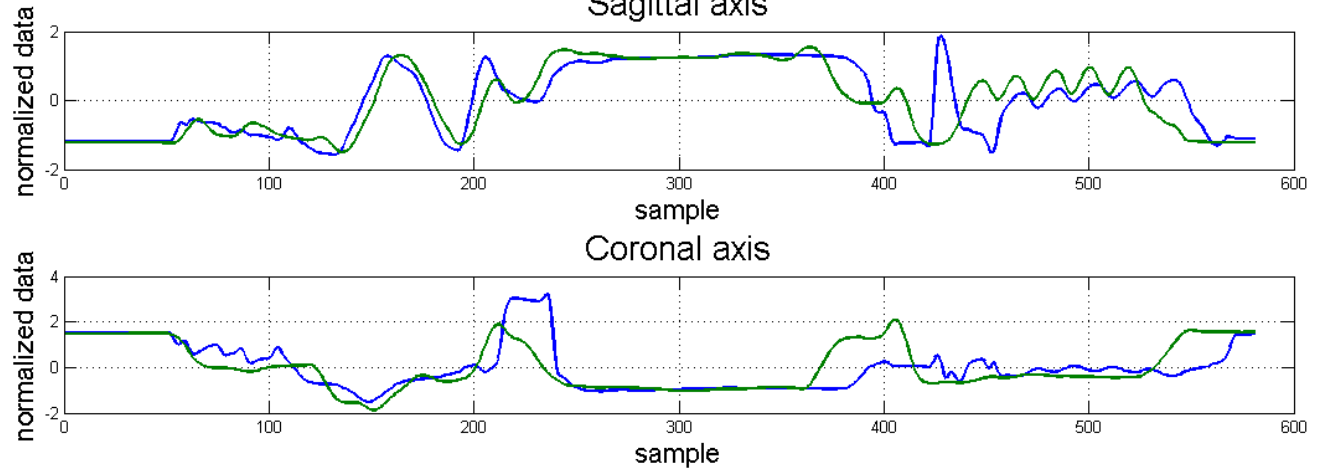

a)
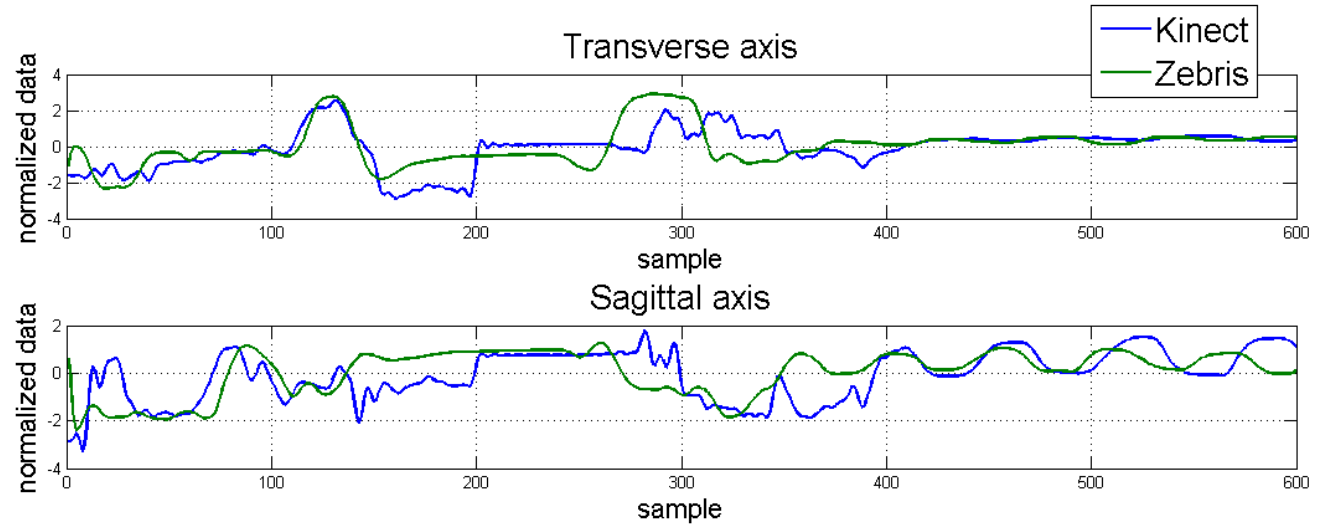

Coronal axis

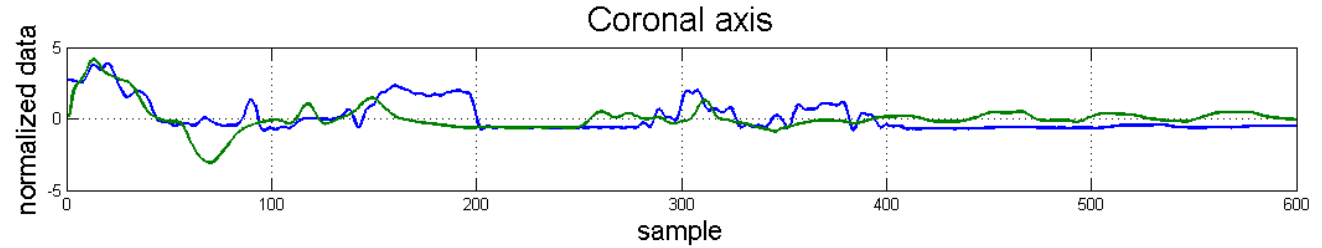

b)

Figure 43. Comparison of position data for the Kinect (blue lines) and the Zebris system (green lines) from a single tea making task trial for the control (a) and the apraxic.

The average Mean Square Error (MSE) and cross-correlation values for each axis are displayed in Table 11. Considering MSE, the values for the control participant ranged from 0.938 to 1.143. The MSE values for the apraxic participant were slightly higher, ranging from 1.265 to 1.322. Statistical analysis indicated that MSE values for the control participant and apraxic patient were significantly different from one another, $\mathrm{F}(1,19)=10.017, \mathrm{p}=$ 0.005. This difference was primarily driven by the MSE values in the sagittal 
axes, which were lower for the control participant than the apraxic patient, $\mathrm{F}(1,19)=4.478, \mathrm{p}=0.018$.

Table 11. Mean Square Error (MSE) and cross-correlation values for the transverse, sagittal, and coronal axes.

\begin{tabular}{|lllllll|}
\hline \multicolumn{3}{c}{ Mean Square Error } & (MSE) & \multicolumn{3}{l|}{ Cross-Correlation } \\
\hline & Transverse & Sagittal & Coronal & Transverse & Sagittal & Coronal \\
\hline Control & 1.143 & 0.938 & 1.074 & 0.427 & 0.489 & 0.525 \\
& $(0.454)$ & $(0.417)$ & $(0.453)$ & $(0.114)$ & $(0.137)$ & $(0.075)$ \\
\hline \multirow{2}{*}{ Apraxic } & 1.265 & 1.322 & 1.292 & 0.367 & 0.338 & 0.353 \\
& $(0.343)$ & $(0.224)$ & $(0.306)$ & $(0.172)$ & $(0.112)$ & $(0.153)$ \\
\hline
\end{tabular}

These results indicate that the overall observation (Kinect ${ }^{\mathrm{TM}}$ ) and estimator values (Zebris) were similar to one another $(\mathrm{p}>0.05)$ regardless of axes, and that the obtained data from the Kinect ${ }^{\mathrm{TM}}$ and Zebris motion capture systems were similar regardless of the neurological status of the participant for the transverse and coronal axes, but were significantly different from another in the sagittal axes.

On the other hand, cross-correlation analysis indicated that the relationship between the Kinect ${ }^{\mathrm{TM}}$ and Zebris systems ranged from 0.427 to 0.525 for the control participant. Based on the Cohen scale [189], these results indicate a medium correlation between motion capture systems for the transverse and sagittal axes, and a strong correlation between motion capture systems for the coronal axis. The cross-correlations for the apraxic patient ranged between 0.338 and 0.367 , indicating a moderate correlation between the tested motion capture systems for all three axes. Statistical analysis indicated that the cross-correlations were similar between the control participant and the apraxic patient. 


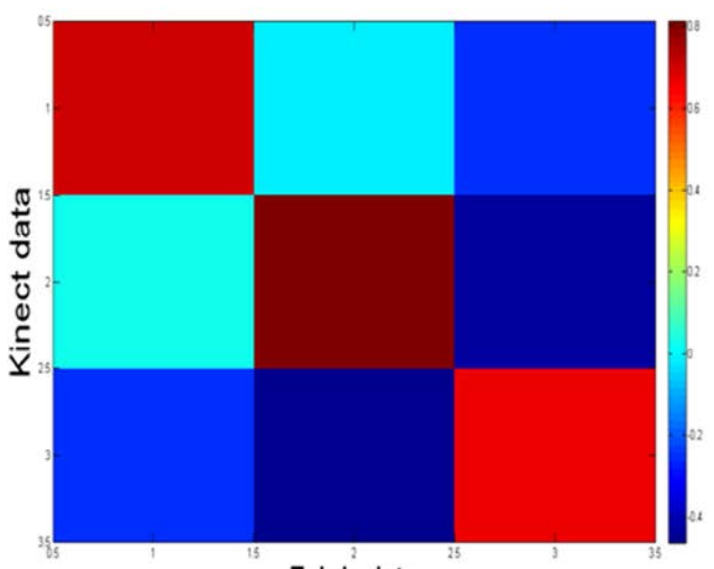

Zebris data

a)

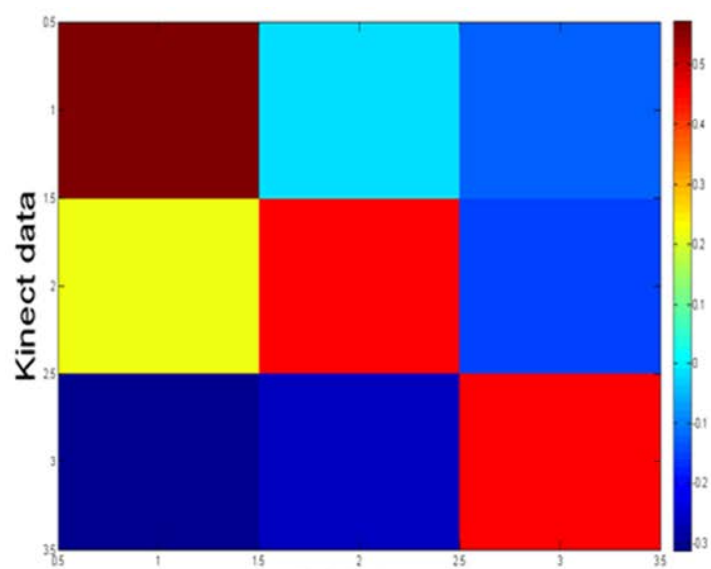

Zebris data

b)

Figure 44. Correlation map between axes for the control participant (left panel) and the apraxic participant (right panel).

Notice from Figure 44 that the correlation map from the trials with the control participant is more symmetric than that one for the apraxic participant due to that the transformation between axes from the Zebris and the Kinect ${ }^{\mathrm{TM}}$ is more linear because of different set up configuration. The data was considered and processed consequently.

\subsubsection{Lessons from monitoring user actions}

The purpose of the current experiment was to ascertain whether the Kinect ${ }^{\mathrm{TM}}$ device can be used as a motion capture system in a cognitive rehabilitative context. Overall, the results indicated a moderate to strong correlation between signals in the control participant, and moderate correlations between signals in the apraxic participant. Furthermore, although the sagittal axes MSE values differed between the control participant and apraxic one, the transverse and coronal axes MSE values were similar for both participants. Taken together, the research presented indicates that the Kinect ${ }^{\mathrm{TM}}$ device is able to adequately track hand movement during ADL, regardless of the neurological status of the individual.

These findings are indeed promising given that the sampling frame of the Kinect $^{\mathrm{TM}}$ device is lower than the Zebris system (30 Hz compared to 120 $\mathrm{Hz}$ ), the position of the hand is determined using RGB camera and depth 
camera, and that the Kinect ${ }^{\mathrm{TM}}$ sensor costs significantly less than its marker-based counterparts.

Moreover, one of the main advantages of the Kinect ${ }^{\mathrm{TM}}$ unit is its continuous development. It expands the possibilities for innovation with features like Near Mode, which enables the depth camera to see objects as close as 40 centimetres in front of the sensor. In addition, up to four Kinect ${ }^{\mathrm{TM}}$ sensors can now be plugged into the same computer and do not need any calibration (if used on Windows). Such possibility is worth mentioning since it can improve and increase the accuracy of tracking and future possible recognition of movements from the kind of patients treated as presented in the thesis. Kinect ${ }^{\mathrm{TM}}$ could also be a useful tool for AR as proven in the results.

\subsection{Reaction time study}

An experiment of response times has been carried out with the aim of study the variability of an electronic system capable of generating pseudo-random visual or acoustic stimuli in random time instants within the interval between stimuli cadence. The use of this system instead of the described interface is necessary in order to accurately measure the Reaction Time (RT). This, together with the biological variability phenomenon would indicate the reproducibility of the system [190]. This study has been carried out in collaboration with Instituto Nacional de Educación Física (INEF).

Certainly, the RT plays an important role in the actions of everyday life as well as sports. The brain requires a minimum of $60-70 \mathrm{~ms}$ for capturing the visual stimulus [191]. After this period the brain can understand and decide an action to perform on response. All these steps, together with the processing time of the measurement instrumentation, would result in TR. While the study of the simple TR could be of interest to study the role of the nervous system, in most cases it has to react to different stimuli that appear without warning, causing a reaction in terms of stimulus and previous experience with an elective-TR [192]

Other objectives of this study are: 
- Assess the consistency and the ratio of the measurements from the RT meter (RTM), see Appendix D.

- Analyse the validity RT meter to detect reliably.

- Determine whether there are differences in the two groups divided by gender when performing a test RT.

- Examine whether the RT to a visual stimulus is faster practitioners in team sports athletes in individual sports athletes.

- Analyse whether competing athletes usually perform better in the RT respect of those who do not.

\subsubsection{Outline}

The methodology used in this study was correlational, comparing the results of RT as sensory pathway (visual and auditory). The RT was studied in a sample, characterized in terms of their gender and their level of sport. Therefore, they cannot establish causal relationships: for example, it is not possible to say that a shorter RT produces a higher competitive level in sport, and vice versa. We can only say, for example, that volunteers with a high competitive level have lower reaction times. This is compared to volunteers of different sports levels in some variables to see if there is a difference between the groups and then validate the measuring apparatus by test - retest. To describe briefly the process that the volunteers have been undergone in this double-blind study typology, the measurements are performed on a single occasion for each volunteer and then volunteers were compared with each other.

\subsubsection{Sample}

The data collection was conducted in the months of December 2013 to March 2014, on the premises of the Centre for Studies on Inclusive Sport (CEDI) of the INEF of Madrid. The study involved 41 volunteers, all INEF students ( X 21.76 years \pm 2.9 ) of the UPM aged between 18 and 35 years. Of these, 30 were men (73\%) and 11 women (27\%). The sample was obtained by random selection procedure. In order to obtain a representative sample of the population of students of the Faculty, gender ratio as close to real for the current academic year, which is $30 \%$ of women in the total it was determined. In relation to the size sample and as 
proposed by the cited authors, an adequate sample number for the population of the Faculty would be 25 volunteers (1,397 men enrolled distributed in 1,075 women and 322 for the year 2012/2013). All participants were presented the study voluntarily after explaining the purpose of it after being informed, they agreed their participation in the study.

\subsubsection{Methodology}

The data collection took place in ideal conditions for carrying out the tests: the experimental context was a quiet place, a table and a chair in which the volunteer was comfortable in front of a computer and the RTM, adequate lighting conditions were settle, without dazzle. The volunteer in sitting position was in front of the computer screen or in front of the RTM device depending on the test, placing in both cases about $40 \mathrm{~cm}$., With the pusher button placed a little closer, at a distance of $30 \mathrm{~cm}$. In our study, each volunteer performed the entire measurement process in a single session. The experimental protocol was as follows:

1. Reception, information and filling out the log sheet.

2. Experiment with the RTM.

3. Break 10'.

4. Experiment with the meter RTM.

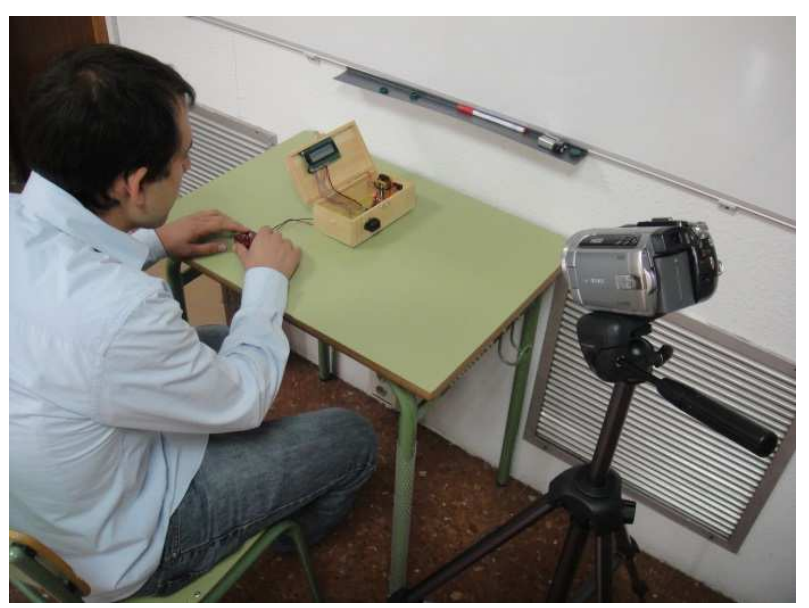

Figure 45. A volunteer doing the test.

The volunteer fulfilled the log sheet before the RT experiment. Then, the volunteer choose a number randomly to determine which one of the six 
available tests to perform in the computer program. The approximate duration of this process was about 5 minutes, enough time for the volunteer to relax and not perform the experiment under stress. It was intended that the volunteer was as calm as possible. Then RT experiment was performed with the computer through an Elective Manual Reaction Time consisting of 8 stimuli, with four visual stimuli and four auditory stimuli randomly interspersed. Before the experiment the volunteer was informed about the next task, also a time to rest was established. Finally, each volunteer dis a pre-test to learn the performance of the system.

Then the volunteer made a break of ten minutes which took place after the second experiment with the same RT meter. During data collection, the placement of the experimenter, the noise of the equipment and other details were controlled to avoid anticipations or loss of attention of the participant. The experimenter stood behind the unit, out of sight of the volunteer and the buttons are pulsed so as not produce any noise.

\subsubsection{Lessons from Reaction Time study}

In order to analyse the distribution of the data and their normality, a Kolmogorov-Smirnov test was applied. This test yielded normal results for each of the variables analysed, so that the parametric statistics study was applied. As descriptive measures the maximum, minimum, average and standard deviation were calculated for each variable. RT for the study of the average of the values obtained in each volunteer by removing outliers avoiding possible errors due to fatigue, forgetfulness, etc. The number of trials was enough to make a stable mean, taking into account that the variability of RT and fatigue did not affect the results. These statistics were studied as a function of the different groups in which the sample was completed: type of stimulus (visual and auditory), gender, type of sport (individual and team sports), depending on the sporting level (if the volunteer competes or do not).

In order to evaluate the possible differences between among the groups according to visual and auditory stimuli as well as to study both instruments, a "T" Student test was performed for comparison of means for related samples. A test " $\mathrm{T}$ " for independent samples was used to 
examine differences by gender, type of sport, and sport level. For all these calculus SPSS 18.0 and Microsoft Excel was used. Significance levels were set to $\alpha<0.05$, indicating the value of $\mathrm{p}$ in each case. From the results and being $0,975 \mathrm{t} 40=2.021$ the null hypothesis, $\mathrm{H} 0$, is not met and may be concluded that the means are equal with a p-value (visual) of 0.001 and pvalue (sound) 0.011. In Table 12, the general results for reaction time are provided.

Table 12. Results of Reaction Time.

\begin{tabular}{lcc}
\hline & \multicolumn{2}{c}{ Reaction Time [ms] } \\
\hline TEST & Visual & Auditive \\
PRE & $0,252 \mathrm{~s} \pm 0.043$ & $0,283 \mathrm{~s} \pm 0.060$ \\
POST & $0,234 \mathrm{~s} \pm 0.030$ & $0.264 \mathrm{~s} \pm 0.047$
\end{tabular}

Table 13. Comparison of means for visual and auditive RT.

\begin{tabular}{l|cc} 
& \multicolumn{3}{c}{ Mean Comparison* } \\
\hline TEST & $\mathrm{r}-$ value $(\mathrm{p}<0.001)$ & T Student \\
\hline Visual & 0.602 & 3.534 \\
Auditive & 0.687 & 2.657
\end{tabular}

RT values obtained are below the values reported by [192]. The ranges obtained are similar to the ones reported by Malapeira et al. [165], between 150-300 milliseconds. The RT for visual stimuli is faster than the times obtained for auditory stimuli as suggested in [193] and contrary to other studies.

\subsection{Monitoring physiological measurements}

It has been described in chapter 2 the role of smart sensing in current state of technology. There is a huge amount of technology for coming out and some companies are placing in market smart watches, wristbands and other devices which have set their focus on the healthcare sector. Prevention to avoid future problems, by promotion of a healthy diet and sport, is the best strategy adopted by these devices. But it is not the only strategy; it is also extended the implementation of a smart health card to always carry with us our medical records and other information relevant for better medical care. 
The future foresights the use of genomics as a core to provide personalized medicine and the inclusion of AI to assists on diagnosis, for example IBMs' agent Watson has been applied to care decision and is generating a new line of research in the field [194].

It is a fact persisting in time that about $75 \%$ of health care budged in developed countries is being spent in chronic diseases. In order to reduce this percentage countries are working on improving the assistance and prevention programs [195].

It is an added value to include physiological measures in a monitoring platform to improve the user experience. From these measurements parameters like fatigue, excitement or stress can be estimated.

\subsubsection{Blood Pressure}

Blood pressure is one of the most important measures between typical measurements by portable devices. The values of BP are tabulated per gender and age and published on the European Guidelines on Cardiovascular Disease[196] it is easy to determine associated chronic diseases like hypertension with the continuous monitoring of this parameter. Another example of the relevance of $\mathrm{BP}$ could be the importance of the follow up BP in post-stroke patient because, as mentioned in [196], significantly reduced new episodes occurs in these highrisk patients by reducing the pressure below 130/80 mmHg (130 mmHg systolic and $80 \mathrm{mmHg}$ diastolic). It would also be important to know the difference between daytime and night-time blood pressure to differentiate dipper patients, which experiment decreases of blood pressure overnight, of non-dipper. Since it is associated with an increased risk from cardiovascular events being a patient of non-dipper type and would benefit from a change in the therapeutic approach, that is, changing medication schedules.

In human-computer interaction $\mathrm{BP}$ can be a parameter for estimating the stress of the patient as several studies demonstrate [197]-[201]. In the evaluation reported on chapter 6 a Non-Invasive Blood Pressure Module (NIBP), see Figure 46, has been used for testing the stress level of the user 
by taking measurement before and after each session. More information about the module can be found in appendix E.

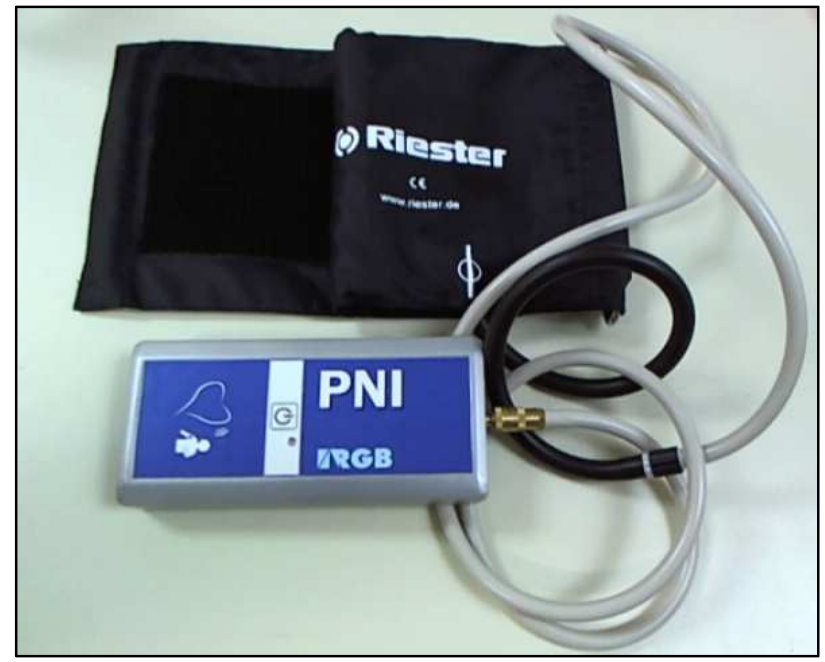

Figure 46. NIBP module from the company RGB.

Additionally, an interface to connect the module to the platform is showed in Figure 47. The interface allows taking measurements of blood pressure. It is a simple interface with three basic actions, start, pause and exit the measure. Subsequently, the data is stored in the local repository organized by session, date and time. For the implementation of this module a library program has been developed in order to manage the Bluetooth protocol and the communication with the device through standard COM ports.

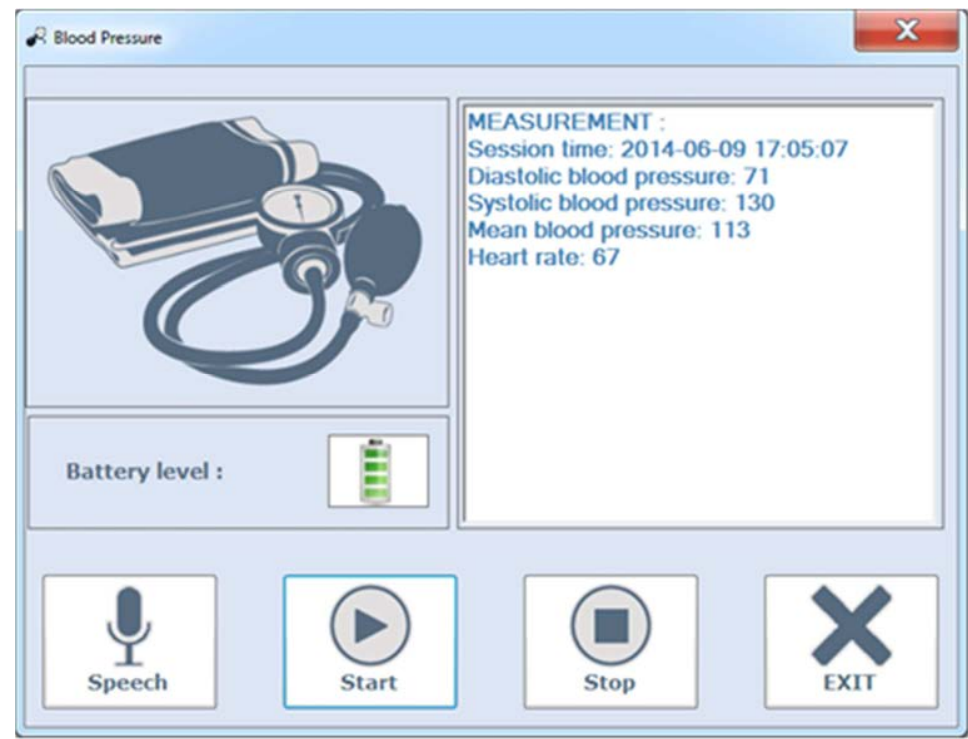

Figure 47. NIBP interface. 


\section{CHAPTER 6}

\section{Evaluation}

This chapter describes the platform where the task model system described in this thesis has been evaluated. After a brief introduction of the system some technical evaluation details are summarized. Finally, the results from an extensive healthcare evaluation are shown to demonstrate the effectiveness of the proposed solution.

\subsection{The CogWatch approach}

The aim of the CogWatch system is to provide cognitive rehabilitation taking into account all the conditions and guidelines mentioned in the literature. For this purpose CogWatch system covers all the following points: assessment of a system specialized for AADS patients, typology of the cues, timing and super-vision of the patient to provide a suitable cognitive rehabilitation in a suitable framework. In the previous chapter it has been described different areas of application for task modelling, To sum up this application are automatic planning, contextualized guidance and monitoring. In this section it will be highlighted that the research and developments carried out in the thesis have turned out to be essential and strong contributions to the outcome prototype of the CogWatch project which has been installed in different environments such as hospitals and other collaborative universities, as indicated in Chapter 1, to provide a real automatic system for cognitive rehabilitation of AADS patients also already introduced in Chapter 2. 


\subsubsection{General system description}

To describe the importance of the contribution exhibited, it is interesting to show the architecture designed for the CogWatch project in collaboration with technical partners from UoB and the LST group from the UPM. In Figure 48, the patient sub-system, conceived for being installed in patients home or rehabilitation centres is illustrated. The Virtual Task Execution (VTE) is the term to denote the system in charge of providing assistance to the patient emulating a "virtual caregiver". As it can be appreciated the start topology of this architecture is governed by a core module named VTE Information Handler. This module is fed with the modules described in this dissertation and an additional Action Recognition module which is out of the scope of this work and has been developed by UoB.

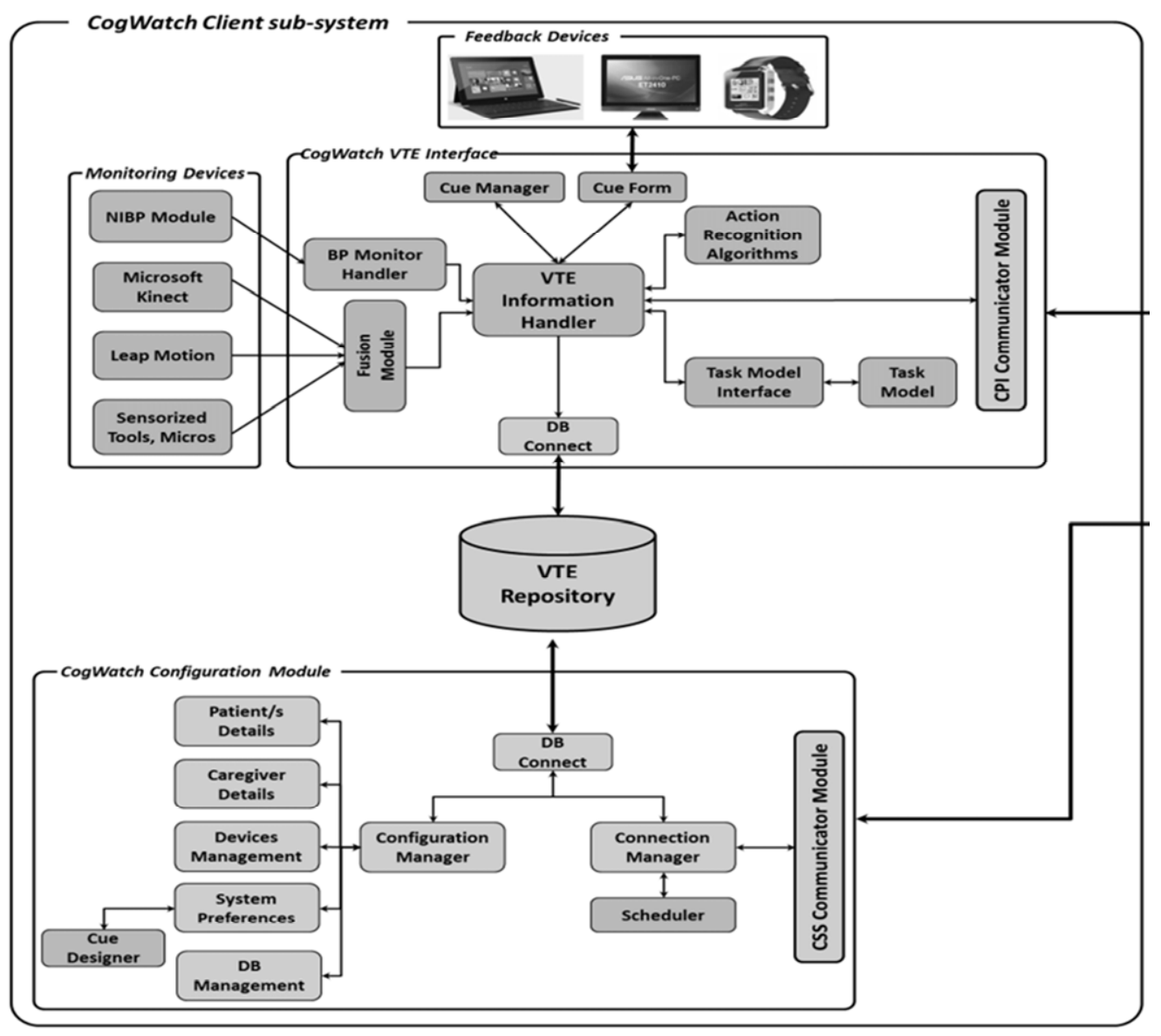

Figure 48. Client- sub system from CogWatch architecture. 
On the other hand, the profile module is included in the configuration system detailed in the bottom of the Figure 48. Also notice that the information of each patient and cues are stored locally in a distributed storage strategy to avoid the problems of an incremental data warehousing avoiding the consequent problems described in chapter 2 .

To provide a detailed description of the client sub-system each of the modules and their functionalities in which the work presented in this thesis has directly contribute are detailed below:

\section{Monitoring Devices:}

Monitoring devices allows the supervision of user movements and attitude as well as the follow up of different signals such as the blood pressure.

The following table, Table 14, sum up the different monitoring devices and their functionalities used in the CogWatch prototypes I and II:

Table 14. Devices for CogWatch prototypes.

\begin{tabular}{|c|c|c|}
\hline Device & Data provided & In support of \\
\hline Kinect $^{\mathrm{TM}}$ & $\begin{array}{c}\text { Coordinates of mouth } \\
\text { points }\end{array}$ & $\begin{array}{l}\text { Detection of toothbrush } \\
\text { approach to mouth }\end{array}$ \\
\hline Leap & $\begin{array}{c}\text { Coordinates from tip of } \\
\text { toothbrush and } \\
\text { hands/fingers }\end{array}$ & $\begin{array}{l}\text { Continuous tracking of } \\
\text { hands while approaching } \\
\text { objects and toothbrush } \\
\text { when grasped }\end{array}$ \\
\hline Shimmer3 & $\begin{array}{c}\text { Accelerations, } \\
\text { orientations and } \\
\text { angular velocities of } \\
\text { wrist }\end{array}$ & $\begin{array}{l}\text { Detection of specific } \\
\text { actions, especially, } \\
\text { toothbrushing }\end{array}$ \\
\hline Coasters & $\begin{array}{c}\text { Accelerations and } \\
\text { forces in sensorized } \\
\text { objects }\end{array}$ & $\begin{array}{l}\text { Detection of motion. } \\
\text { Recognition of actions }\end{array}$ \\
\hline Microphones & $\begin{array}{l}\text { Featured sound in } \\
\text { mouth and toothbrush }\end{array}$ & $\begin{array}{l}\text { Behaviour during } \\
\text { toothbrushing }\end{array}$ \\
\hline
\end{tabular}




\section{Fusion module:}

The data provided by Kinect ${ }^{\mathrm{TM}}$ and Leap are combined and presynchronized in order to provide such data to the Fusion Module, so to consider the data source as a unique device.

The wireless connection manager, in the meantime, is principally focused on obtaining the data from the sensorised objects, which contain the coasters, through the Bluetooth communication, hence processing and synchronizing the information.

Finally, all data is synchronized and combined at the final stage with the data provided by the Shimmer3 sensor, in order to provide useful and reliable information to be used by the VTE Information Handler and to be sent to the corresponding algorithms for the action recognition and the error detection. The data is stored in a specific log files in the Data Base repository.

\section{Blood Pressure Monitor Handler}

The system has been previously described in the section 5.4.1. The communication is established through a Bluetooth module compatible with the standard 2.0 which emulates the behavior of an asynchronous serial channel. This module is classified as "class 1 " so it provides a maximum range of 100 meters without obstacles.

The process used during a normal session to monitor the vital signs of the patient is as follows:

- The patient turns on the sensor module.

- After that, the sensor module activates Bluetooth permanently and the connection from the Gateway is established.

- The Gateway arranges the start of the session using the desired functioning mode.

- The sensor module transmits the corresponding information from the patient according to the mode selected. 
- The session is considered as finished when the module is turned off or when the corresponding order to do that is sent from the Gateway.

\section{Cue Manager}

The Cue Manager (CM) is the module in charge of localizing among the internal multimedia files the one most appropriate to show to the patient. Furthermore, there are different environmental constraints which the system must take into account. Constraints are set by the way in which the patient is used to perform an action. For this reason a number of different factors determine the behaviour of the system. These factors include:

- Time required for the patient to perform an action.

- The progress among the global task.

- Repeatability in the way of performing a task.

- User preferences.

- The way in which the information is shown to the patient.

\section{Cue Form}

It has been developed an interface, see Figure 49, which supports all the communication channels: described in section 4.3, the restriction from section 4.4 and the considerations from section 4.5 .

The interface is showed as a black screen with three interaction buttons and an exit button to allow the user to abort the session at any time. These buttons are aligned aside the cue screen considering Fittss' law[166] to prevail the interaction with the system. 


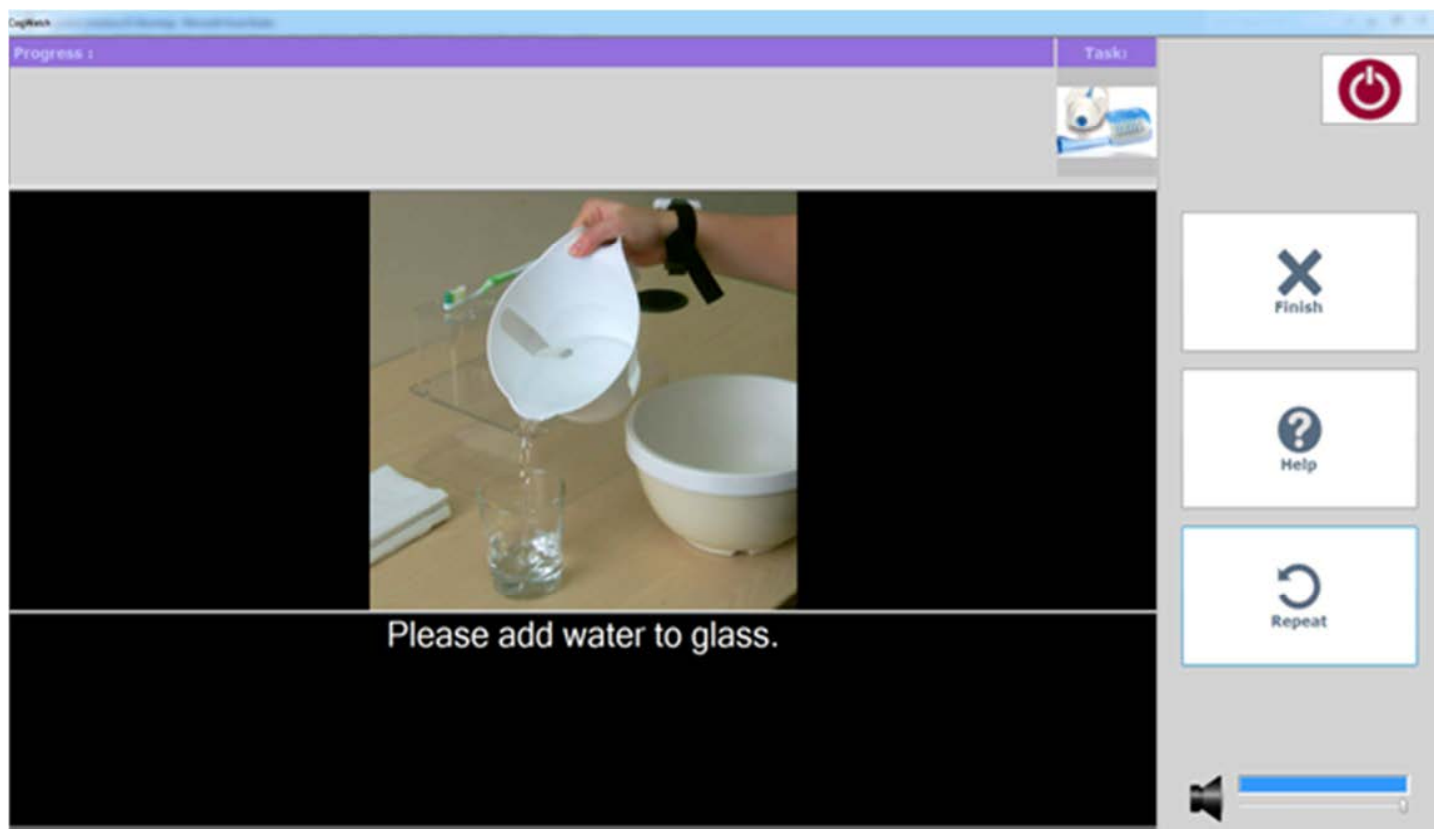

Figure 49. Appearance of the cue interface.

These buttons provides the following functionalities:

- Finish button: the user must press the button if she/he considers that the session has finished.

- Help button: the user can press it to request some assistance from the system.

- Repeat button: the user can press it to repeat the last displayed cue.

Additionally, Cue Form provides different layouts to define some additional cues to measure general progress during the performing of a task. These cues are:

- Cue Progress: a portrait view of the correct sub-actions done by the patient represented by a still.

- Cue Goal: a still of the final state of the task to remind the session objective to the patient.

The developed platform provides cues in English, German and Spanish. The problem is extensible to the forms from the interface.

\section{Information Handler}

The IH module manages the communication with the following modules: 
- Fusion Module, in charge of the data link from the monitoring devices.

- Action Recognition Algorithms and Task Model, focused on the recognition and the prediction of the action and the errors of the user.

- VTE Cue Manager, focused on managing the cues to prompt the user.

- Blood Pressure Module, in charge of providing data related to vital signs of patient, such as the heart rate and the blood pressure.

- Data Base repository. This module has a specific permission to exchange information with the Data Base Repository when required, especially, focused on the connection of devices and the relevant data of patient that may affect the performance of the cue prompting.

In addition, the $\mathrm{IH}$ is in charge of assigning priorities in order to protect the system from collapse due to overload. Considering the communication with the Fusion Module, the IH starts the connection with the monitoring devices and consequently the writing of the recording files that are stored in the Data Base once the session has terminated.

Then, the IH acts as a linker to send the raw data, derived from the Fusion Module, to the corresponding Action Recognition Algorithms. The output from these algorithms is successively sent to the Task Model to determine whether an error has been committed. The information about the error committed is obtained in terms of a specific code that allows the system to know what cue is needed, based on the information stored in the corresponding XML file.

\subsubsection{Prototypes set-up}

Two ADL has been considered during the development of the project to demonstrate the efficiency of the system in the assistance of ADLs. These selected activities are tea making and tooth brushing which represent two of the five task categories of ADL to manage basic self-care reported in [202], meal preparation and grooming activities respectively. The tables defining actions and errors from the study of each task analysis can be consulted in appendix F. 


\section{Tea preparation}

The following set.-up was prepared for the experiment of tea preparation, see Figure 50

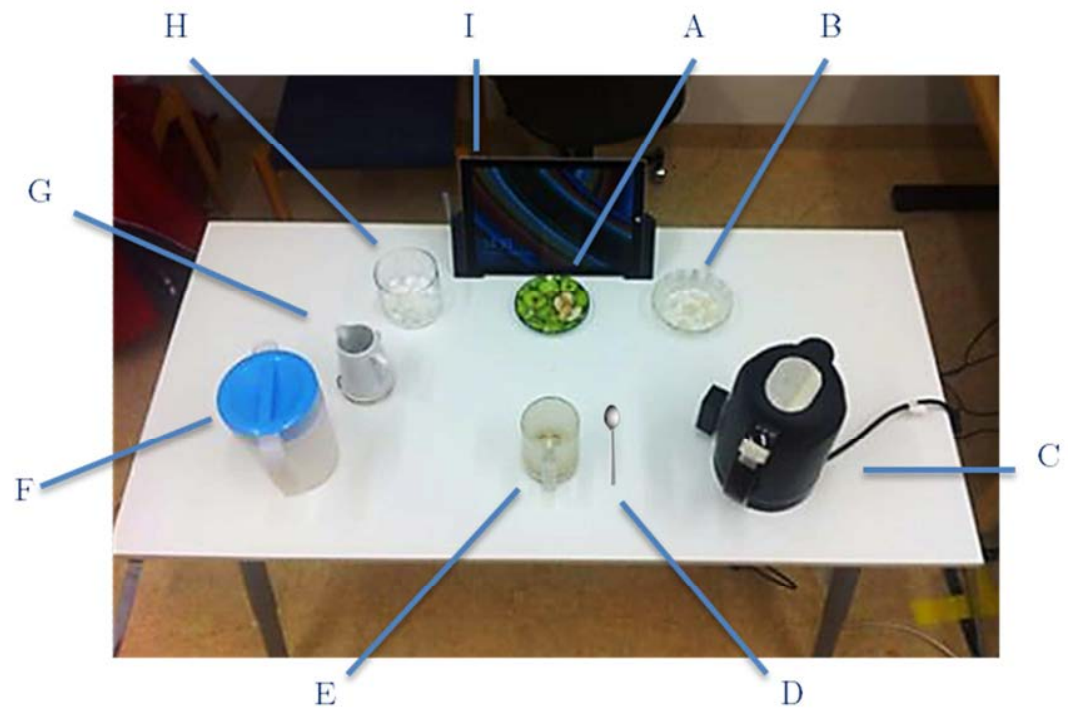

Figure 50. Layout for tea preparation.

The material for this experiment is listed below:
A. Teabags container
B. Used teabags container
C. Kettle
D. Spoon
E. Mug
F. Jug of water
G. Jug of milk
H. Sugar container
I. VTE Monitor (Tablet pc)

\section{Tooth brushing}

Like in the first experiment the following set.-up was prepared for the experiment of tooth brushing, see Figure 51. 


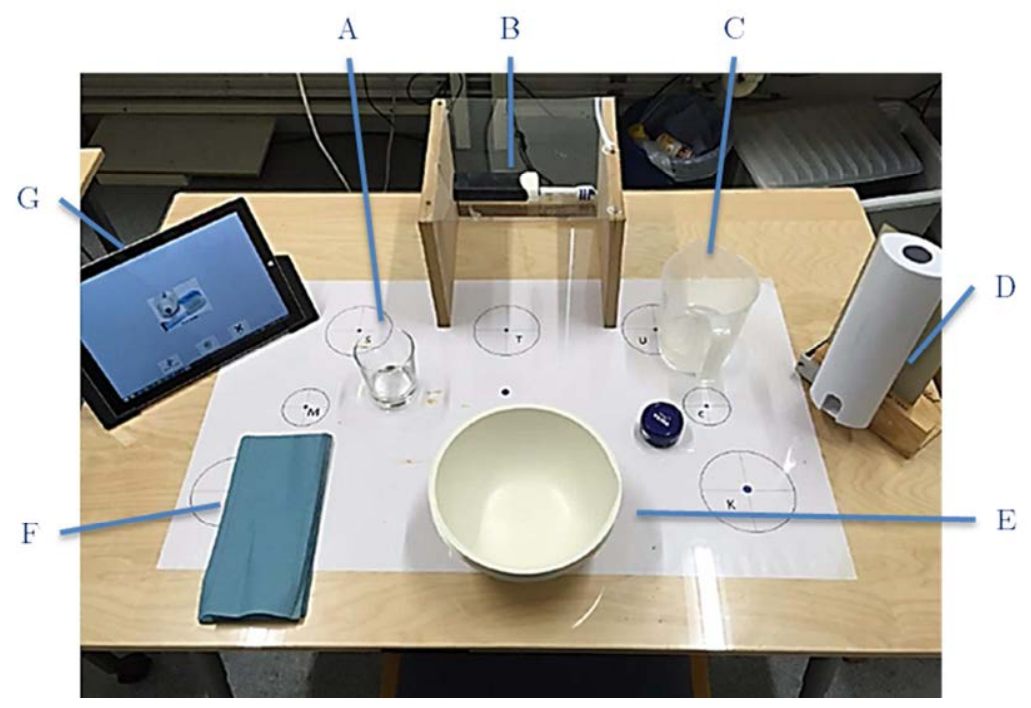

Figure 51. Layout for toothbursihing.

The material for this experiment is listed below:
A. Glass of water
B. Tooth brush
C. Jug of water
D. Toothpaste dispenser
E. Spittoon
F. Paper napkin
G. VTE Monitor (Tablet pc) 


\subsection{Technical evaluation}

The aim of the technical evaluation was to improve the prototype in a continuous quality cycle. The inner purpose is to provide a prototype to assess rehabilitation at the home of the patient. This is a very ambitious objective an a technical validation is considered as a first step necessary for moving from the laboratory prototype towards a home based environment, the idea is illustrated on Figure 52.

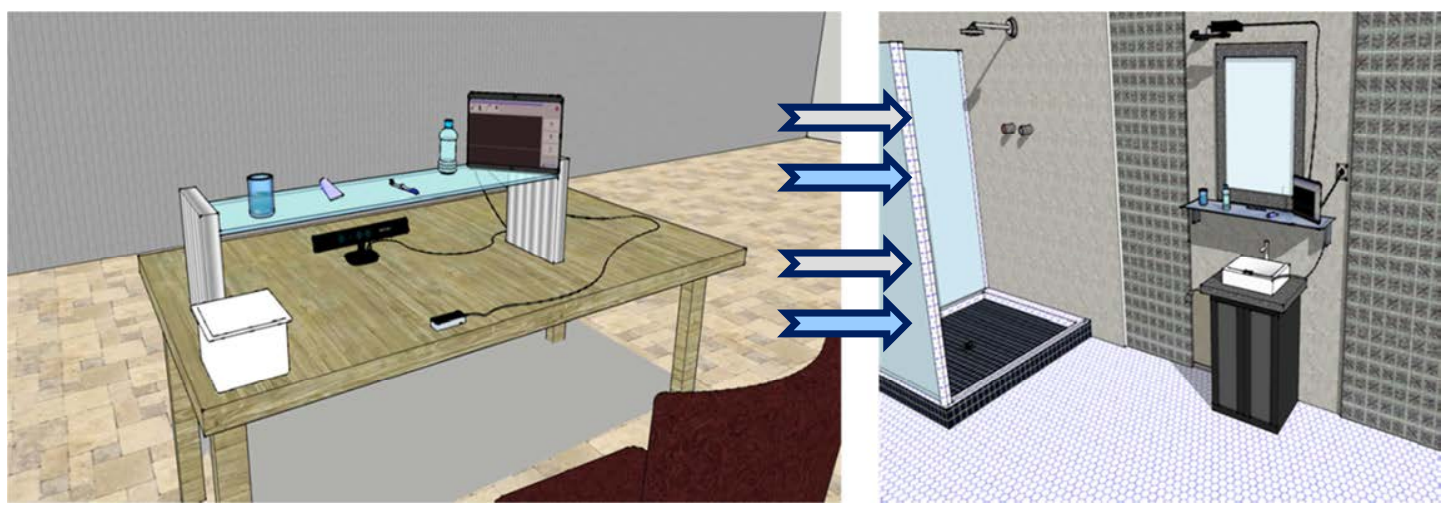

Figure 52. The quality step of technical validation.

In order to ensure proper operation of the prototype extensive technical validation was performed, it is out of the scope of this thesis to describe all the processes but it is necessary to highlight and mention briefly the work carried out necessary for the further validations described in this chapter. Some of the test performed consisting of:

- Unit testing of software components

- Interactive design

- Usability tests

- Monkey tests

- Connectivity tests

○ time delays

- load tests

- latency of connections 


\subsection{Health care evaluation}

\subsubsection{The opinion of stakeholders}

Headwise and The Stroke Association carried out different studies on United Kingdom population. Several groups were run across the West Midlands including Bromsgrove, Solihull and University of Birmingham. The groups started with an explanation of the CogWatch solution, after that an in-vivo demonstration of the actual P1 system was performed or a video of the prototype was shown instead. For all groups in relation to P2 the tools were demonstrated and participants were able to hold and comment on them and videos of any cues were presented on a small screen. Open ended questions were developed to give participants free rein to raise issues relevant to the evaluation. A total of 36 users and 6 carers took part in the focus groups where 31 had a diagnosis of a stroke and 5 of brain Injury. 16 out of the 31 participants were users who were seeing CogWatch for the first time.

The study highlights:

- Users and carers felt that at present there were technical constrains to the system would be ready for a home environment in terms of the amount and cost of the sensors. Suggestions were made on whether the current prompts could be downloaded on to a tablet or even technology that a user may already have in order to try and reduce potential cost.

- The therapists were concerned regarding how compatible this system would be with current restrictions on IT within hospitals and data protection.

- All groups felt that a lot of training and continuous support especially in terms of technical issues would be required.

- In terms of task preference a high percentage of users still feel that it is important for them to be independent in personal care, where carers and therapists still feel that kitchen tasks are where the focus for independence should be.

- Overall, both the therapist and users felt that CogWatch would be excellent as a rehabilitation tool within the hospital setting. 
It is worth to mention that both groups agreed that further technical developments needed before the system is ready to be used in current clinical and home environments but the conceptual solution presented was considered excellent.

Regarding the cues some general comments were:

- Cues needed to be visual: "need to be simple", "have one to two words underneath describing", "to have clear simple pictures".

- Videos would be much easier and clearer for an individual to follow.

- For auditory prompts, ten users commented that they would prefer this type of cue but all felt that these needed to be "individualised in terms of voice and language used".

- Combination of cues and prompts is excellent especially for those who have 'forgotten how to do tasks' or ' needed prompts to move on to next step' and that having the different types could actually mean that in some way the system could be personalised for each individual.

\subsubsection{Prototype I: tea making}

The evaluation of the prototype 1 aims to compare this prototype with standard procedures in rehabilitation in occupational therapy. The experiments and results presented here are carried out and obtained in collaboration with the Technische Universität München (TUM). The aim of this study is to measure the usability and efficiency of the CogWatch solution. For this reason, the following experiment was made.

\section{Objectives}

The first objective of this study is to confirm if the automatized assistance provided by the prototype is good enough to conduct a patient to carry out the task of preparing a cup of tea.

A second objective is to measure the effectiveness of a simple recipe book for assisting a patient to successfully make the same task of preparing a requested cup of tea. 
The third objective is to determine if exists any significant different between the use of the prototype and the recipe book.

Finally, the most important objective is to determine the immediate effects from both methods.

\section{Sample}

During this study 21 patients with Left and Right Brain Damage (LBD \& RBD) were selected to perform this test after as screening stage. The results from the screening guarantee that every patient manifested any of the apraxic symptoms with related errors on ADL performing. Patients selected were in the final stage of the course of their disease (corresponding to phases C \& D of the German classification of stroke severity). This sample is considered representative for the objectives of the study.

\section{Material}

The same tasks were required to the patient independently of using the prototype or the recipe books, patients were request to perform the four available types of tea included in the prototype. To wit: Black Tea (BT), a tea with no additional ingredients, Black Tea with Sugar (BTS), White Tea (WT), BT with milk and White Tea with Sugar (WTS).

The recipe books consist on an illustrated book, see Figure 53, with the same still cues from the prototype ordered in a correct sequence to complete the task.. All the patients received an explanation of the book. They could use them as a guide going through the task step by step or look up single sub-steps at will. The caregiver provided no further help. 


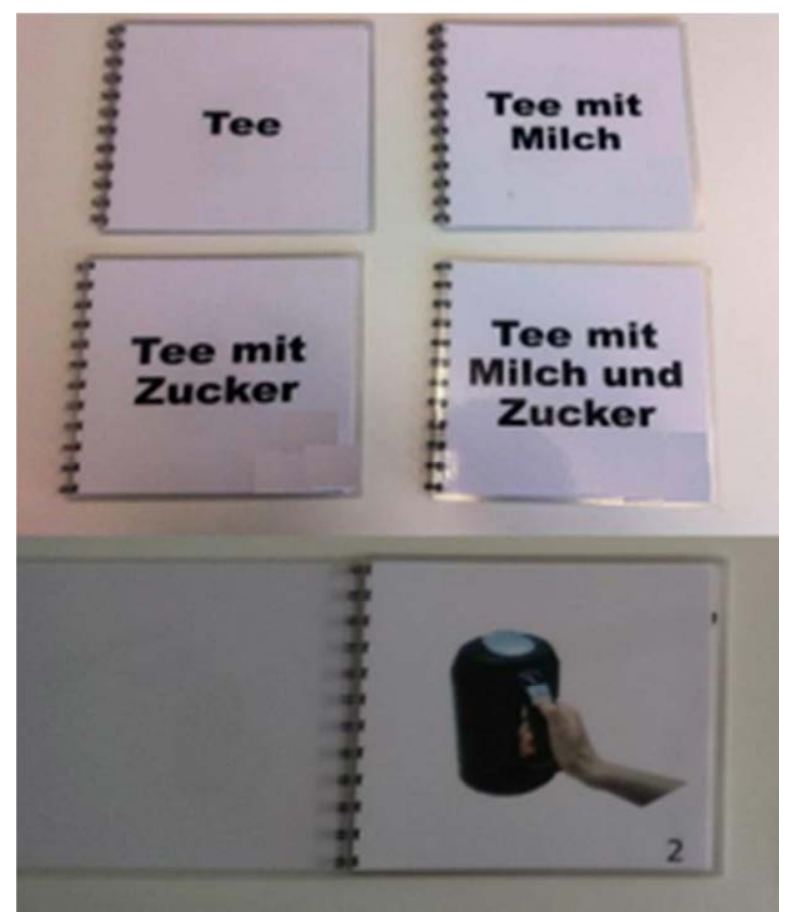

Figure 53. German version of recipe-books.

\section{Methodology}

A comparative study requires a particular topology. It is for this reason that a crossover design for the experiment was performed. The process followed by this design is showed on Figure 54. The process is described as follows:

The first baseline is set at the beginning of the experiment. The first session is chosen randomly to avoid conditioning the study. During the session, all patients prepared all four different cups of tea in a random order also. Immediate effects are tested after the session for the first follow-up milestone. Then, the patient was invited to repeat the test within the following 6 days with at least 24 hours between both sessions. In the second test after testing the conditions in the second baseline the patient carried out the opposite experiment in the same manner as the first one. The session included a Baseline and Follow-up tea-making task that occurred at the start and the end of each session by performing a WTS, due to the higher complexity of this task among the other types of tea preparation. 


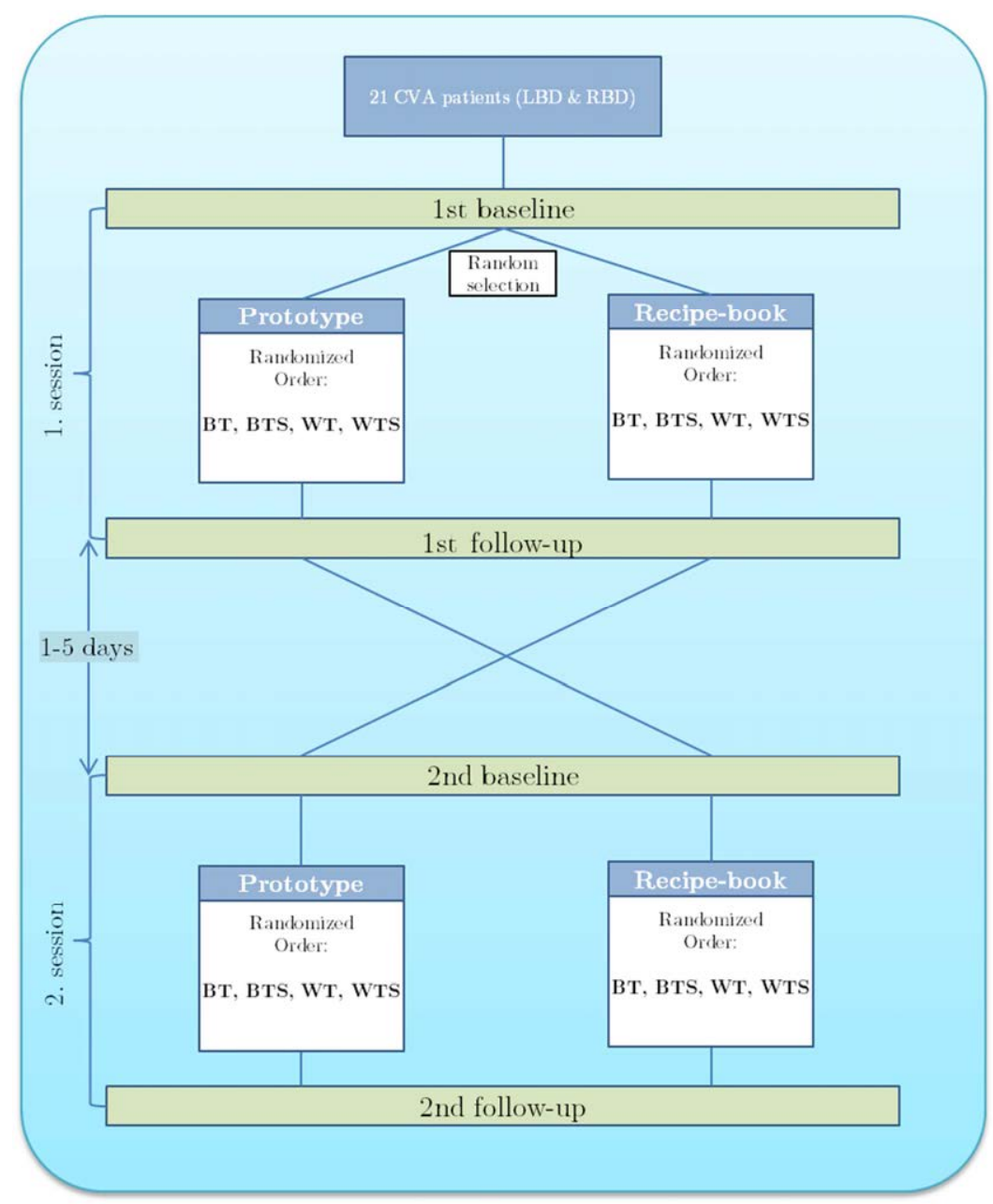

Figure 54. Crossover design for prototype I experiment.

To analyse the results several McNemar test studies has being realised [203]. The outcomes from this test allow contrasting the hypothesis formulated between both strategies as well as between each session and each type of tea. McNemar's test is considered the most suitable procedure for the dichotomous nature of the study and the pre and post conditions. Additionally, a parallel study was applied to the committed errors. The error data was analysed using paired t-tests in order to analyse the differences in the average number of errors produced in each of the six tea trials in both conditions.

\section{Results and discussion}

It is important to consider the differences between both processes to understand the results obtained in the test. On the one hand, during the prototype session, the patient only received assistance in the form of error 
cues when required, this is, when an error were committed or the patient asked the system for the next step, see appendix $\mathrm{F}$ for more information on the errors covered by the prototype. On the other hand, the second strategy, recipe book), the patient, instead of getting a cue when needed, had to check the book by themselves and manually look for the next correct step. The use of guide books is widely extended in therapy (e.g. aphasic patients for communication), so, it can be assumed that they are familiarized with this strategy. In addition to this, the layout of the Tea-Books was similar to what was displayed on the prototype interface and in both cases; the caregiver provided no feedback to the patient.

The percentage of successful teas made with the prototype is showed in Table 15, manifested an improvement in the performance of the task.

Table 15. Successful test percentage (\%) with prototype.

\begin{tabular}{|c|c|c|c|c|c|}
\hline Baseline & BT & BTS & WT & WTS & Follow-up \\
\hline 29 & 90 & 90 & 90 & 95 & 48 \\
\hline
\end{tabular}

The analysis of $\mathrm{p}$ values from McNemar test concludes that, no significant different between the baseline and the follow up was found ( $\mathrm{p}-0.22)$. When compared the tea trials with the baseline, the results suggested that the teas made assisted by the prototype were more likely to be successfully completed (with $\mathrm{p}<0.01$ ). The results comparing the tea trials and the follow up revealed also a better performance with the prototype $(\mathrm{p}<0.05)$.

On the contrary, the percentage of successfully made teas in the recipe book case indicated no improvement in the performance of the task with this strategy, see Table 16.

Table 16. Successful test percentage (\%) with recipe book.

\begin{tabular}{|c|c|c|c|c|c|}
\hline Baseline & BT & BTS & WT & WTS & Follow-up \\
\hline 29 & 38 & 38 & 24 & 29 & 19 \\
\hline
\end{tabular}


The results from the McNemar test obtained in this case not found any significant difference between the baseline and the follow-up ( $p>0.05)$. Also report no significance difference between the baseline and each of the 4 tea trials while performing the task with the recipe book $(\mathrm{p}>0.05)$. Finally, no differences were found too between the follow up and each of the four tea trials using the McNemar tests $(\mathrm{p}>0.05)$.

It was also desired to compare both kinds of strategies (prototype and recipe book) between them. For that purpose additional comparisons using McNemar test were made. The results from the comparison between both baselines report no significance different $(p=1.0)$ meanwhile the comparison between the follow up test corroborate the hypothesis that more successful teas were obtained at the follow up with the prototype strategy $(p=0.0)$. Finally, for all the four tea making trials the result from the recipe book were significantly worse than the results from the prototype case, $(\mathrm{p}<0.01)$. To summarize the results, once settled the statistical significance of them Figure 54 displays the successful tea data for each patient across all of the trials for each condition.

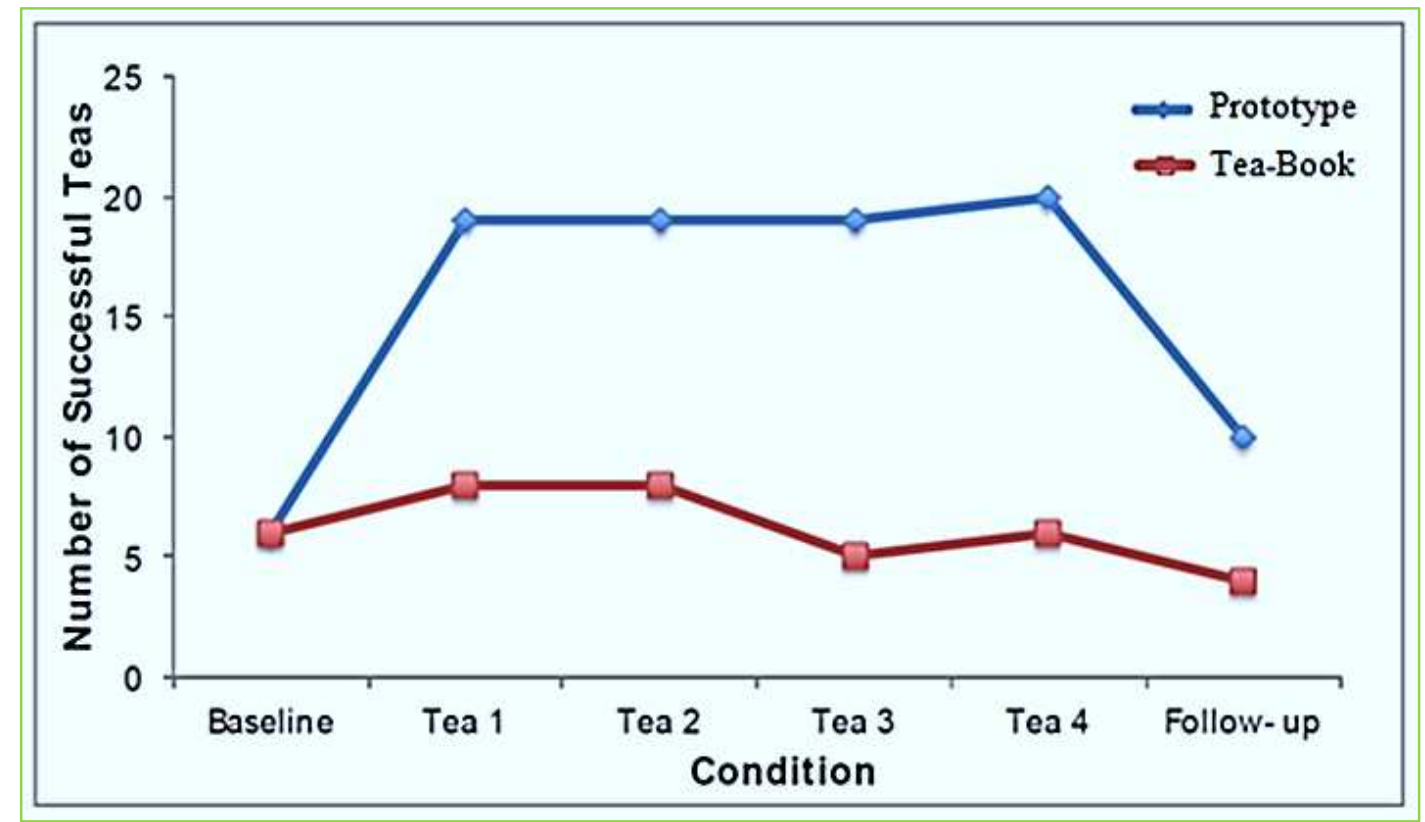

Figure 55. Number of successful teas made by patients in the six stages of the study. 


\subsubsection{Prototype II: tooth brushing}

The clinical evaluation of the prototype II reports a study carried out in collaboration with the UoB. To validate the performance of the prototype in a grooming task and in second term, record useful data for improving the task model, the analysis of apraxic patient during the task of tooth brushing was accomplished. This data collection considered a small group of 5 patients. The registers and data obtained was useful in order to established a basis for further studies with larger population and to register different sequences in the realisation of the task to improve the available task model by the incorporation of these users statistics.

The patients were required to perform the task of brushing their tongue/teeth in the same manner they would at home, including duration. The sessions were video recorded, under the consent of the patient, for later analysis and marking. Each of the 5 patients performed a total of three trials with rests between them. During the rests, carers rinsed the bowl the glass and the toothbrush as well as they added fresh water to the jug. The patients performed the trials without additional instructions from the carer, whilst the clinician inputted the order of completed steps and supervises the cues displayed by the prototype. The trials were recorded for offline scoring. Results are presented in Table 17 where the sequence of steps and errors made for each patient are shown. 
Table 17. Order of the sequences performed by each patient; shadowed in red the steps performed after cueing.

\begin{tabular}{|c|c|c|c|c|c|c|c|c|c|c|}
\hline & & $\begin{array}{l}\text { Add } \\
\text { water }\end{array}$ & $\begin{array}{l}\text { Add } \\
\text { toothpaste }\end{array}$ & $\begin{array}{l}\text { Wet } \\
\text { brush }\end{array}$ & $\begin{array}{l}\text { Brush } \\
\text { teeth }\end{array}$ & Rinse & Spit & $\begin{array}{l}\text { Clean } \\
\text { brush }\end{array}$ & $\begin{array}{l}\text { Wipe } \\
\text { mouth }\end{array}$ & $\begin{array}{l}\text { Empty } \\
\text { glass }\end{array}$ \\
\hline \multirow{3}{*}{$\begin{array}{c}\text { Patient } \\
1\end{array}$} & Trial 1 & 1 & 3 & 2 & 4 & 5 & 6 & 8 & 7 & 9 \\
\hline & Trial 2 & $1 ; 8$ & 3 & 2 & 4 & 5 & 6 & 9 & $7 ; 10$ & 11 \\
\hline & Trial 3 & 1 & 2 & & 3 & 4 & 5 & 7 & $6 ; 9$ & 8 \\
\hline \multirow{3}{*}{$\begin{array}{c}\text { Patient } \\
2\end{array}$} & Trial 1 & 1 & 2 & 3 & 4 & 7 & $5 ; 8$ & 6 & $9 ; 11$ & 10 \\
\hline & Trial 2 & 1 & 2 & 3 & 4 & 7 & $5 ; 9$ & 6 & 8 & 10 \\
\hline & Trial 3 & 1 & 2 & 3 & $4 ; 7$ & 10 & $5 ; 8 ; 11$ & 6 & $9 ; 12 ; 14$ & 13 \\
\hline \multirow{3}{*}{$\begin{array}{c}\text { Patient } \\
3\end{array}$} & Trial 1 & 1 & 2 & & 3 & 4 & 5 & 6 & 8 & 7 \\
\hline & Trial 2 & 3 & 1 & & 2 & $4 ; 7$ & $5 ; 8$ & 6 & 9 & 10 \\
\hline & Trial 3 & 1 & 2 & & 3 & 5 & 4 & 6 & 7 & 8 \\
\hline \multirow{3}{*}{$\begin{array}{c}\text { Patient } \\
4\end{array}$} & Trial 1 & 1 & 2 & $3 ; 5 ; 7 ; 10$ & $4 ; 6 ; 9$ & $1 ; 12 ; 14$ & $8 ; 11 ; 13$ & 16 & 18 & 17 \\
\hline & Trial 2 & 1 & 2 & & 3 & $6 ; 8$ & $5 ; 7 ; 9$ & 4 & 11 & 10 \\
\hline & Trial 3 & 1 & 2 & & 3 & $6 ; 8 ; 11$ & $5 ; 7 ; 10 ; 13$ & $4 ; 9$ & 14 & 12 \\
\hline \multirow{3}{*}{$\begin{array}{c}\text { Patient } \\
5\end{array}$} & Trial 1 & 1 & 2 & & 3 & 7 & $4 ; 8$ & 5 & $6 ; 9$ & 10 \\
\hline & Trial 2 & 1 & 2 & & 3 & 6 & $4 ; 7$ & 5 & 8 & 9 \\
\hline & Trial 3 & 1 & 2 & & 3 & 6 & $4 ; 7$ & 5 & 8 & 9 \\
\hline
\end{tabular}

- Patient 1 committed two omission errors in trial 1 (actions 7.Clean brush, 9. Empty glass into bowl.) and another in trial 2 (9.Empty glass into bowl), while performed the third correctly. 
- Patient 3 committed one omission error in trial 1 (8.Wipe mouth) another in trial 2 (9.Empty glass into bowl) and two errors in trial 3 (8.Wipe mouth, 9. Empty glass into bowl).

- Meanwhile, Patient 2, 4 and 5 did not commit any error.

Figure 56 displays the time that each patient took to brush their teeth. The measurements where considered from the insertion of the brush into the mouth until the toothbrush exited the mouth. Where the patient stopped brushing, wetted the brush or spat into the bowl before continuing brushing (e.g. patient 4, trial 1), brushing time reflects the sum total of the time the brush was in the mouth.

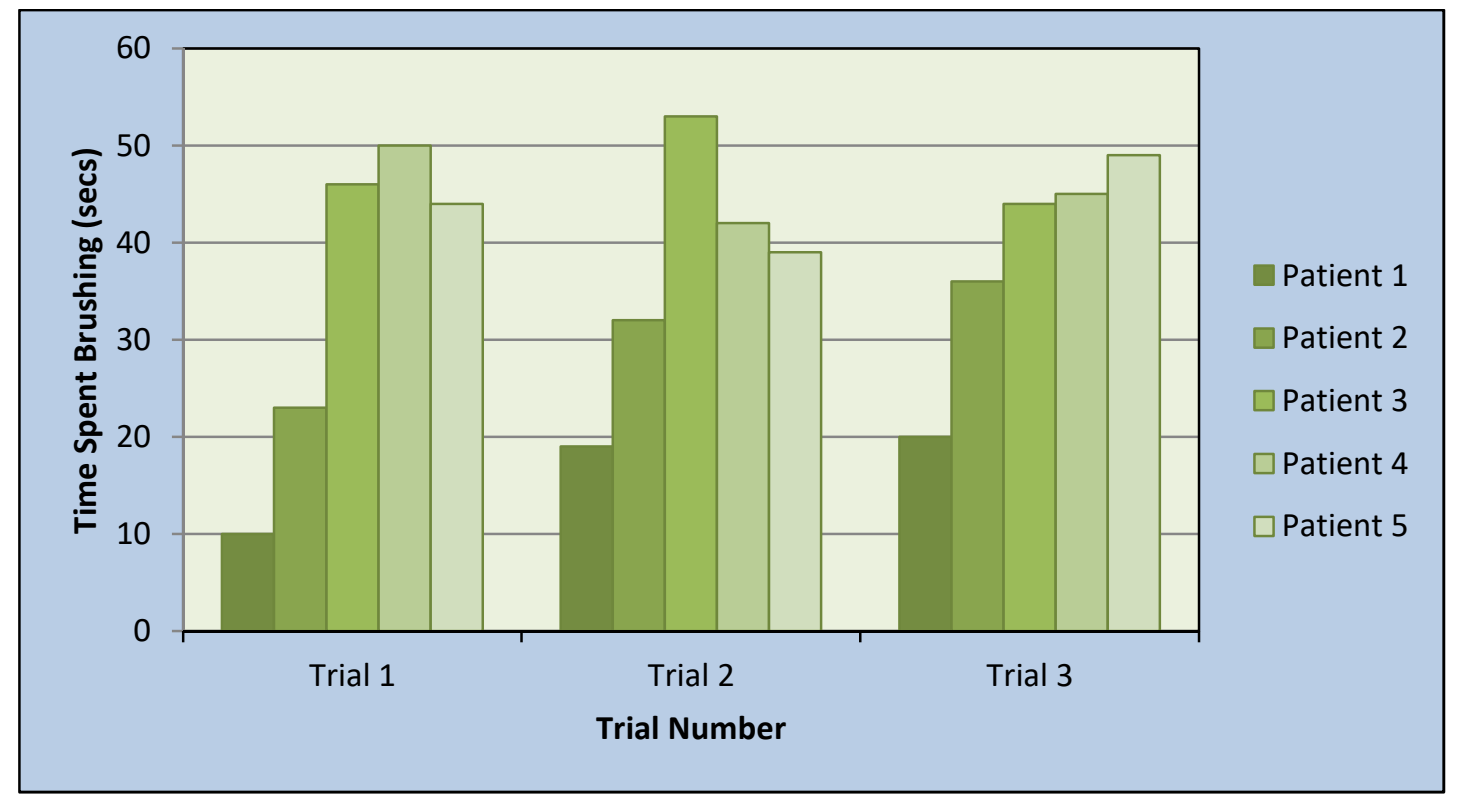

Figure 56. Time spent by each patient brushing their teeth across the three trials.

Regarding the patient feedback, the user interface was clear and easy to use. Patients would use the system in their own home. The patients stated that they did not know how long to brush their teeth for despite explicit instruction to brush for as long as they would do at home.

\subsubsection{Induced stress}

8 patients from the efficacy trial carried out in UoB underwent a study with the NIBP interface described in the previous chapter, chapter 5 . Measurements were taken during each of the 5 weekly training sessions, before and after making 8 cups of tea with CogWatch prototype. To avoid 
the white collar effect a maximum of 5 measurements were registered for each patient. Table 18 summarizes the average values of heart rate, systolic and diastolic BP of the population studied.

Table 18. Average values of NIBP experiment.

\begin{tabular}{|l|l|l|}
\cline { 2 - 3 } \multicolumn{1}{c|}{} & Average heart rate & Average BP \\
\hline Pre-CW & $67 \mathrm{bpm}$ & $117 / 62 \mathrm{mmHg}$ \\
\hline Post-CW & $67 \mathrm{bpm}$ & $116 / 58 \mathrm{mmHg}$ \\
\hline
\end{tabular}

The general results demonstrate that both measurements from both pre and post sessions are similar, what suggest that the patients did not suffer under stress during the trials as the study of the difference of means concludes. The average results from this study are shown in Figure 57. (Notice that mean $\mathrm{BP}$ refers to the mean $\mathrm{BP}$ registered during the blowing of the module and not the mean of systolic and diastolic BPs).

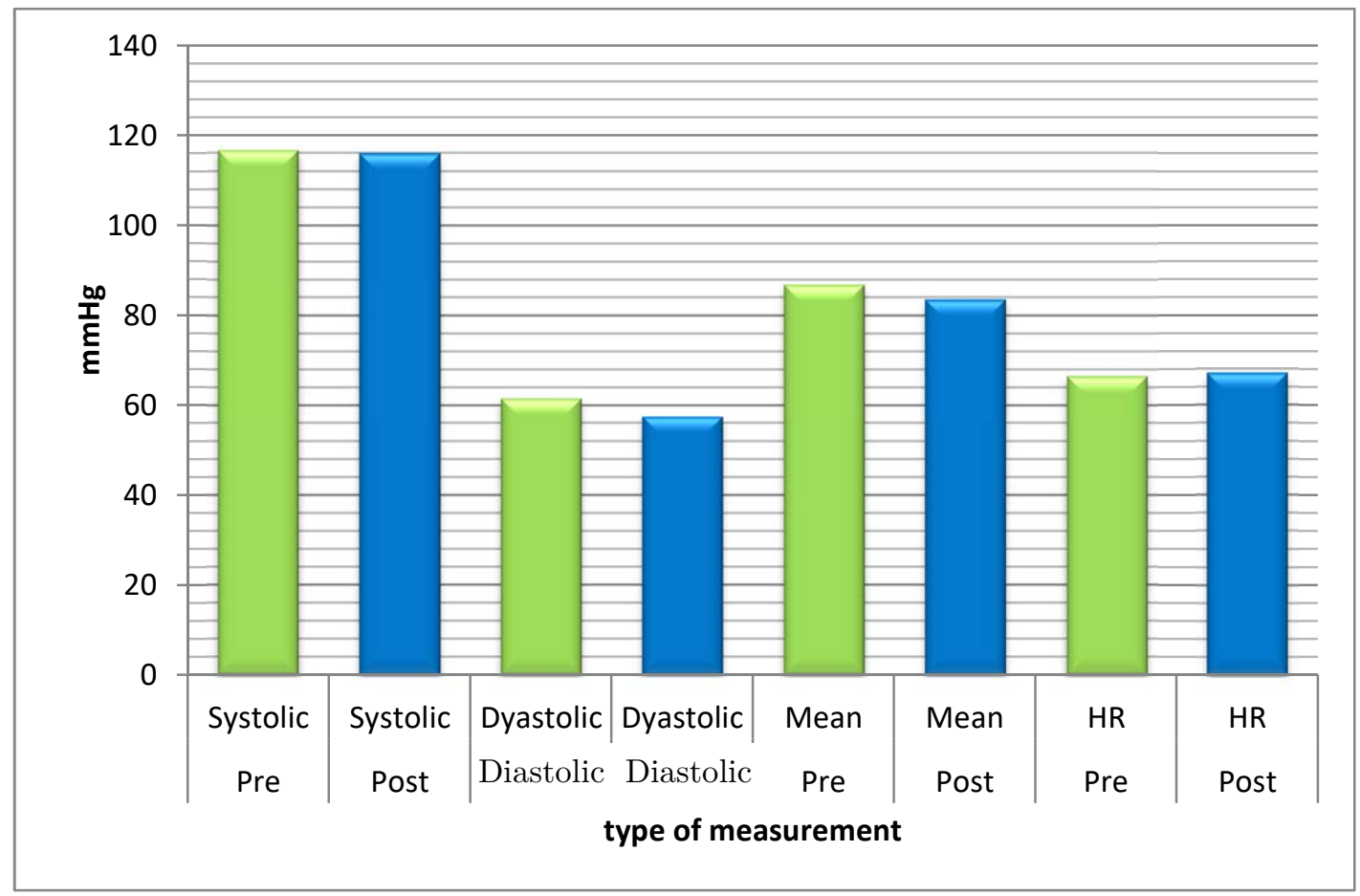

Figure 57. Results from UoB efficacy trial. 


\subsection{Discussion}

The evaluation described in this chapter aims to manifest the effectiveness of the techniques described in this thesis as a core contribution for an ICTbased rehabilitation platform. The platform has been demonstrated as an effective tool for the assessment of guidance and support for patients of AADS in two representative ADL tasks, a hot drink preparation and a grooming task. The number of successfully accomplished sessions, where the task were completed by the patient, reveal that the system is appropriately for supporting cognitive rehabilitation. It is important to notice that, even when conditions were not significantly different from the baseline, the system was still able to successfully guide patients through the multi-step tasks and reach a successful outcome.

From interviews with focus groups it has been conclude that the line followed in this research is a valid approach. Another discussion that arises from this evaluation is the benefits of providing the information when the user requires it, instead of presenting general guidelines. The results from the evaluation suggested that the first strategy prevails retain information in subsequent sessions.

The cognitive errors more frequently committed were those related to omission errors, as described by the literature, the system performs well capturing these errors. The prototype addresses the difficulties experimented by AADS patients by removing the amount of cognitive load placed on them. The technology supports via feedback the patient so she/he only has to concern about performing the task.

These conclusions from the experts are positive valuations which reinforces the work carried out. To cite some:

"Feedback on the system was very positive. All patients recognized the value of the system and stated that they would use the system in their own home." "This flexibility of the system is vital for its success in the home-based setting by ensuring that it can adapt the level of support provided depending on the ability of patients. This adaptability of the system is evident in the results of individual patients." 


\section{CHAPTER 7}

\section{Conclusion}

This final chapter remarks the conclusions from the experience gained during the realization of this thesis. From the framework of chaos theory S. Kauffman described the unpredictability of complex systems [204]. This behaviour has been widely appreciated in different chaotic systems, such as the well know Lorenz attractor [205]. Our cortex is an amazing field of research, the regions of the cortex involved in different cognitive processes are well defined but injuries in different spots of the brain, even if those spots are very close, could lead in extremely different manifestations and impairments. But, as S. Kauffman suggested, is in this tiny line which divides the severity of a clinical manifestation where the capacity of human beings is manifested. We can move forward and prevail over our environment

Adaptability is the key of our evolution and keeps its' basis on behaviour. It is impossible to predict human behaviour as we have the willingness to modify it and to adapt it to our circumstances. The proposed solution constitutes a step forward in assistance and relies on ICTs. At the beginning of this work, a reflection on how we use the information and whether it is appropriate arises. An indirect conclusion was that the responsibility for a person to learn depends on the use of the information available. The information changes our behaviour and therefore our chances to overcome the rehabilitation process. 


\subsection{Conclusions}

While a user executes a task, it can be monitored in real time using traditional methods of reinforcement learning. But to be effective requires some level of expertise. A good strategy to provide that level of expertise is obtained by providing the system with greater flexibility. For that purpose it must be taken into account user preferences. The solution showed in this dissertation has been demonstrated as an effective Task Model strategy for acquiring an adaptive behaviour of the system by combining a MDP based solution with a model checking solution and defining the proper interaction between them.

For a proper definition of a task model two requirements are essential:

First, it is necessary to properly define the hierarchies and actions, as well as all the possible errors involved in the task. It could lead in high dimensional spaces and is in this stage where the help of an expert is necessary. The fundamental role of the expert concerns to define the error table and the required level of segmentation via a stopping rule and every tool for obtaining that information supposes an additional value.

Second, it is necessary to define multiple models of a task to obtain an adaptive response from the system. A useful adaptive response can be obtained by the combination of quality and quantity preferences of the user.

Multimodal guidance is more effective when it considers different levels of assistance as far as the user evolves in learning. For this purpose it is essential to store relevant information of the results of every session. Also a solution for characterizing the learning factor through an algorithm for selecting the proper model is important.

Regarding the integration of the described solution for a more complete prototype under the framework of the CogWatch project it could be concluded that the solution has been demonstrated to help to reduce errors committed by AADS patients and to be effective to assess cognitive rehabilitation. Some requirements have to be faced in order to fulfil user suggestions for a home based platform such as the inclusion of more ADLs and the optimization of the apparatus for reducing effective costs. 
In order to determine some of the challenges found in the implementation of the platform it has been found some constrains and limitations which must be considered to reproduce and improve the work reported in this thesis:

- To achieve feasible action recognition it was required to undergo a large process of data acquisition and algorithms refinement. The best strategy to deal with this problematic is the emulation of the recognition stage through tele-supervision via professional intervention. This solution requires that the professional inputs the actions made by the user remotely. This system must be blind from the front-end perspective to obtain useful data for the final product.

- Action recognition via Hidden Markov Models does not allow the early recognition of actions and, therefore, prospective cueing was not tested.

- Larger studies and data collection are required to carry out inferential statistics. All the conclusions extracted from this study are limited to descriptive statistics. Future works will consider larger test beds and the development of a commercial ready product which can be used at home by patients.

- Technological constraints are, currently, a leap to surpass by society, emerging technologies such as IoT foresights a further impact of this work in consonant of technological trends in the near future. This results in a lower impact and less rejection of the technology from the user.

Both, users and carers agreed that one of the advantages of the system is the flexibility to allow personalizing the cues and adapt it to their necessities and capacities. It can be concluded that the solution adopted by CogWatch improves current rehabilitation techniques and the model exposed could become a new guideline in personalized rehabilitation.

The work presented aims to demonstrate how technology could be applied to enhance our way of living and in particular the advantages that task modelling provides to the three main fields of application: taking decision, provide contextualized guidance and monitoring. 
First, it has been presented a MDP based task model for helping an actor to take decisions. The manifested shortcomings of the system has been solve by complementing the solution by the adoption of a distributed system which combines MDP and model checking, the description of the system shows how both strategies can interact with each other.

Second, the information from the task model has been applied to define a feedback system which takes advantage of the concurrent state to contextualize the information showed to the user improving the communication and interaction with the user.

Third, an approximation to provide user monitoring has been presented and validated. The results from this approach demonstrate that i) a non-invasive strategy is suitable for assisting the user through a sequential multi-step task and ii) the user is not induced under stress according to parametric physiological signals.

Finally, according to the objectives set at the begging of this dissertation it can be conclude that:

- The problematic and requirements for modelling the activities and errors made by users has been reviewed.

- A model for monitoring performance of actions in multi-step tasks has been presented. Furthermore, the model has been implemented and tested.

- Special attention has been payed to provide useful feedback to users during the execution of multi-step tasks.

- Thanks to the CogWatch project[2], the work carried out during this thesis has been evaluated in a promising tool for the rehabilitation of AADS patients.

\subsection{Future Work}

The work carried out during this researching period of time opens a discussion about the usefulness of the task model strategy. In particular the work presented proposes a new line of action in the field of cognitive rehabilitation. This type of rehabilitation has been somewhat ignored and is 
currently acquiring more importance. Care needs described by the patients have been translated to different solutions. The strategy followed in patients of AADS can be borrowed in other syndromes as dementia with high incidence among the elderly population.

Due to time restrictions the influence of a prospective and retrospective cueing strategy has not been studied and the platform described could be used as a suitable testing suite. All the modifications needed are briefly described in chapter 4 to carry out this work.

The strategy demonstrated for preparing a hot drink and grooming can be extended to other ADLs. The major restrictions are focussed on the recognition of actions and the instrumentation required for affording it, but, the gradual introduction of sensorised objects in the market provides new solutions to this matter.

Finally, due to the scope of the research project, the work described is only available in a prototype status and further work must be done in order to guarantee effective cost and simplicity to install the system in a domestic environment with all the advantages that rehabilitation at home could bring. Personalization has been proved to be a key factor for enhancing the system and all future work on this topic will be welcomed by both carers and patients.

In the following section some of the incoming technologies that currently and in a near future will provide new solutions for personalized home care are cited. The underneath idea is to justify the instauration of new technologies at home against the posture that claims about them as unnecessary invaders. The benefits from these technologies as well as their drawbacks are analysed.

\subsubsection{Future lines of research}

The methodology exposed in this thesis could be applied to multiple areas of research. It can take advantages of the increasing electronic instrumentation of the denominated "smart" objects and the IoT. Furthermore, collected information will make it possible to generate personal profiles based on $\mathrm{BD}$ and generate target alerts for desired 
indicators, all managed via an internal Task Model. There is a wide field of application for the work presented. To cite some of these possible areas:

- Public services :

Some management services such as hospitalization, the use of public transport or administrative processes can be supervised and automatized individually to avoid conflicts and streamline these services.

- Healthcare :

Personalized healthcare improves the quality of life of people and different alerts systems could be designed using the methodology reported.

- Guidance systems :

Instruction sheets could be replaced by multimedia systems conceived for mobile platforms which could be more efficient and ecologically sustainable.

- Manufacturing

Some manufacturing systems, such as lean manufacturing, based some of their efficacy indicators in avoid risk. Task Modelling could be applied to detect possible risks in the manufacturing process. 


\section{Appendix A}

\section{Publications}

The list of publications published and dissemination activities held during the realization of this thesis are detailed:

\section{Journal Articles:}

1. Cogollor, J. M., Pastorino, M., Rojo, J., Fioravanti, A., Wing, A., Arredondo, M. T., Ferre, M., Breñosa, J., Hermsdörfer, J., De Teresa, J., Walton, C., Worthington, A., and Giachritsis, C. An Innovative Solution Based On Human-computer Interaction to Support Cognitive Rehabilitation. Journal of Accessibility and Design for All, vol. 4 (3), pp. 238-254, 2014.

2. Pastorino, M., Fioravanti, A., Arredondo, M. T., Cogollor, J. M., Rojo, J., Ferre, M., Bienkiewicz, M., Hermsdörfer, J., Fringi, E., and Wing, A. Preliminary Evaluation of a Personal Healthcare System Prototype for Cognitive eRehabilitation in a Living Assistance Domain. MDPI Sensors, vol. 14, pp. 10213-10233, 2014.

3. Cogollor, J. M., Hughes, C., Ferre, M., Rojo, J., Hermsdörfer, J., Wing, A., and Campo, S. Handmade Task Tracking Applied to Cognitive Rehabilitation. MDPI Sensors, vol. 12, pp. 14214-14231, 2012.

\section{Conference proceedings:}

1. Cogollor, J. M., Campo, S., Rojo, J., Ferre, M., and Aracil, R. A Novel Cognitive Rehabilitation Based on Virtual Reality Techniques. IEEE Intelligent Vehicles 2012, Workshop on Perception in Robotics. $3^{\text {rd }}$ $7^{\text {th }}$ June 2012, Alcalá de Henares, Madrid, Spain.

2. Rojo, J., Cogollor, J. M., Pastorino, M., Fioravanti, A., Breñosa, J., Arredondo, M. T., Ferre, M., Aracil, R., and Sebastián y Zúñiga, J. M. Sistema de Rehabilitación Cognitiva para la Asistencia en Actividades Cotidianas de Pacientes tras Sufrir un 
Accidente Cerebro-vascular. Robótica Cognitiva 2013, $12^{\text {o }}$ Workshop Robocity 2030. $4^{\text {th }}$ July 2013, Madrid, Spain.

3. Cogollor, J. M., Pastorino, M., Rojo, J., Fioravanti, A., Wing, A., Arredondo, M. T., Ferre, M., Breñosa, J., Hermsdörfer, J., De Teresa, J., Walton, C., Worthington, A., and Giachritsis, C. An Innovative Solution Based On Human-computer Interaction to Support Cognitive Rehabilitation. V International Congress on Design, Research Networks, and Technology for all 2013. $23^{\text {rd }}-25^{\text {th }}$ September 2013, Madrid, Spain.

4. Pflügler, Johannes, Andrea Schlegel, Emilie Jean-Baptiste, Pia Rotshtein, Matteo Pastorino, Javier Rojo, Jose Maria Cogollor, Maria Teresa Arredondo, Marta MN Bieńkiewicz, and Joachim Hermsdörfer. "Using Human-Computer Interface for Rehabilitation of Activities of Daily Living (ADL) in Stroke Patients: Lessons from the First Prototype." In Replace, Repair, Restore, Relieve-Bridging Clinical and Engineering Solutions in Neurorehabilitation, pp. 629-636. Springer International Publishing, 2014.

5. Rojo-Lacal J.I, Soto-Rey,J, Perez-Tejero,J Rojo-González,J.J. "Aleatory system for obtaining visual and auditory response time." 2014, EUCAPA, European Congress of Adapted Physical Activity. Monday, September 29, 2014 to Thursday, October 02, 2014, INEF, Madrid, Spain.

6. Gómez, M., Rojo, J, Cogollor, J. M., Sánchez-Urán, M. A., Sebastián, J. M., Ferre, M., and Aracil, R. IR Based Perception System for Evaluation of Human Hand Manipulation. IEEE Humanoids 2014, Workshop on Cognition, Perception and Postural Control for Humanoids. $18^{\text {th }}$ November 2014, Madrid, Spain.

\section{Contribution to the Organization of Workshops and Conferences:}

1. IEEE Humanoids 2014, Workshop on Cognition, Perception and Postural Control for Humanoids. 18th November 2014, Madrid, Spain.

2. Cumbre de Robótica para los Ciudadanos en la Comunidad de Madrid. 27th September 2013, Leganés, Madrid, Spain. 


\section{ApPEndix B}

\section{Algorithms FOR DEALING With UNCERTAINTY IN INPUT ACTIONS}

POMDP algorithm, [144]

\section{Inputs:}

A: set of machines' action a

$\mathbf{Q}(\mathbf{b}, \mathbf{a})$ : expected cost for selecting a when in $\mathrm{b}$

$\mathbf{N}(\mathbf{b}, \mathbf{a})$ : number of times a is selected when in $\mathrm{b}$

$\mathbf{B}_{\mathrm{S}}$ : initial set of belief states $\mathrm{b}$

п: initial policy, with $\pi: \mathrm{B}_{S} \rightarrow \mathrm{A}$

repeat

$\mathrm{k} \leftarrow 0$

$\mathrm{b}=\mathrm{b}_{0}$ (probability 1 to be in the initial state $\mathrm{s}_{0}=\varnothing$ )

Generate a tea trial with the Simulated User:

repeat

$\mathrm{k} \leftarrow \mathrm{k}+1$

Get Simulated Users' output $\mathrm{a}_{\mathrm{u}, \mathrm{k}}$ and update $\mathrm{b}$

$\mathrm{a}_{\mathrm{k}} \leftarrow \Pi(\mathrm{b})$ or $\Pi\left(\mathrm{b}_{\mathrm{n}}\right)$

with $\mathrm{b}_{\mathrm{n}} \leftarrow$ nearest neighbour of $\mathrm{b}$ in $\mathrm{B}_{\mathrm{s}}$

$\operatorname{record}\left(b_{k}, a_{k}, c\left(b_{k}, a_{k}\right)\right), K \leftarrow k$

until

Scan trial, update $\mathrm{b}$ and $\mathrm{Q}$

for $\mathrm{r} \boldsymbol{\epsilon}[0, \mathrm{~K}] \mathrm{do}$

$\mathrm{C} \leftarrow$ sum of the costs $c\left(b_{k}, a_{k}\right)$ from $b_{r}$ to $b_{K}$

if $\exists \mathrm{br} \in \mathrm{B}_{\mathrm{s}}$ and $|\mathrm{bk}-\mathrm{br}|<\mathrm{e}$ then

$$
\begin{aligned}
& \mathrm{Q}\left(\mathrm{b}_{\mathrm{r}}, \mathrm{a}_{\mathrm{r}}\right) \leftarrow \frac{\mathbf{Q}\left(\mathbf{b}_{\mathrm{r}}, \mathbf{a}_{\mathbf{r}}\right) * \mathbf{N}\left(\mathbf{b}_{\mathrm{r}}, \mathbf{a}_{\mathbf{r}}\right)+\mathrm{C}}{\mathbf{Q}\left(\mathbf{b}_{\mathbf{r}}, \mathbf{a}_{\mathbf{r}}\right)+\mathbf{1}} \\
& \mathrm{N}\left(\mathrm{b}_{\mathrm{r}}, \mathrm{a}_{\mathrm{r}}\right) \leftarrow \mathrm{N}\left(\mathrm{b}_{\mathrm{r}}, \mathrm{a}_{\mathrm{r}}\right)+1
\end{aligned}
$$

else

create a new point $b_{w}$ in $B_{s}$ 
Appendix B

initialise $\mathrm{Q}(\mathrm{bw}, \mathrm{ar}) \leftarrow \mathrm{C}(\mathrm{bw}, \mathrm{ak})$

end if

end for

update policy

$\left(\mathrm{b}_{\mathrm{r}}\right)=\operatorname{argmina} \mathrm{Q}\left(\mathrm{b}_{\mathrm{r}}, \mathrm{a}_{\mathrm{r}}\right)$ foreach br $\boldsymbol{\epsilon} \mathrm{B}_{\mathrm{s}}$, foreach $\mathrm{a}_{\mathrm{r}} \boldsymbol{\epsilon} \mathrm{A}$.

until converged 


\section{ApPendix C}

\section{TASK MODEL COMMUNICATION TESTS}

Program and tests results.

\section{Results from Information Handler to Task Module}

The following code was used for doing the stress test.

For the client side:

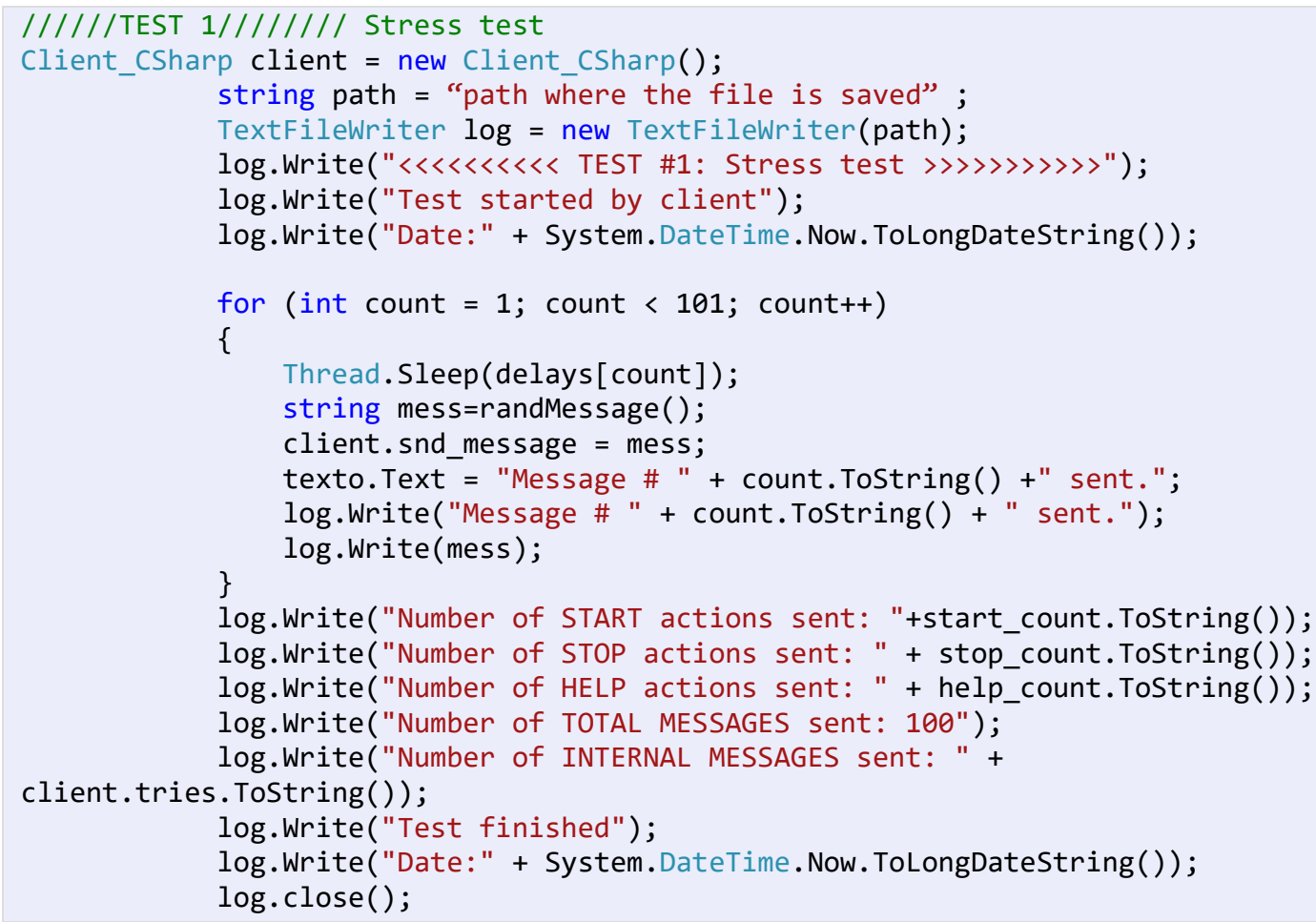


And for the server side:

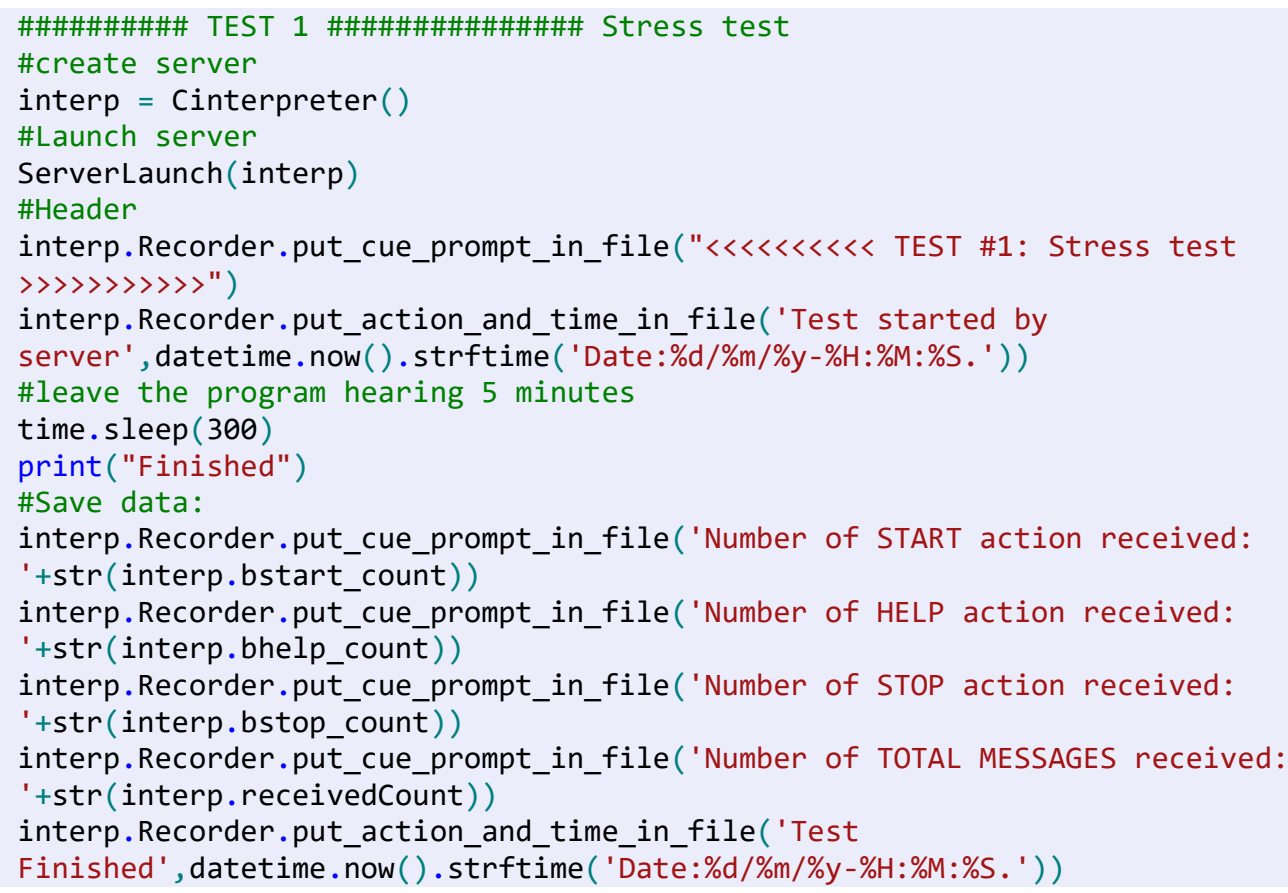

Stress testing results were success. Hundred per cent of message sent from client were received from the server but the system requires 10 trials for sending the last message what slows down the communications in this channel. These results were improved by shortening the size of the message with a compression method.

The following code for doing the timing test were used

For the client side:

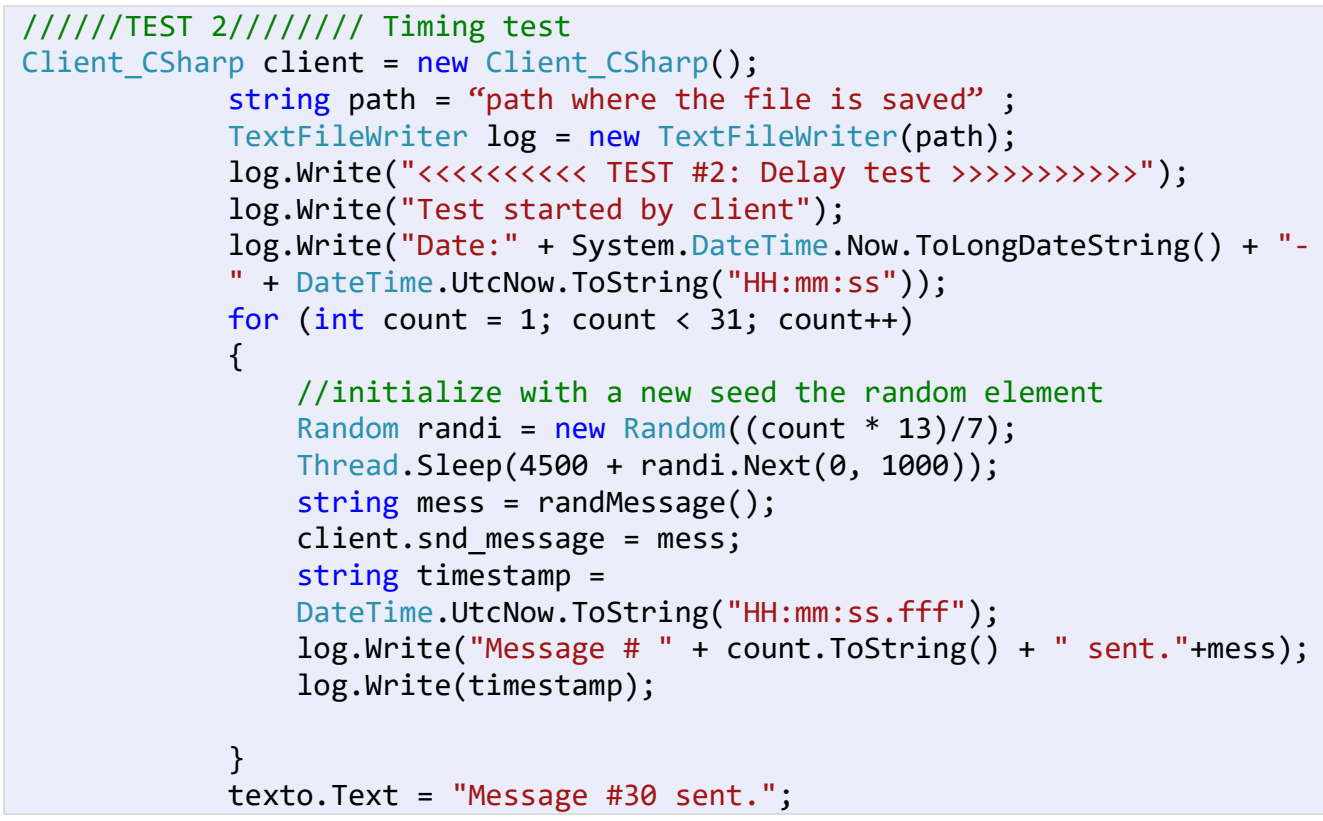




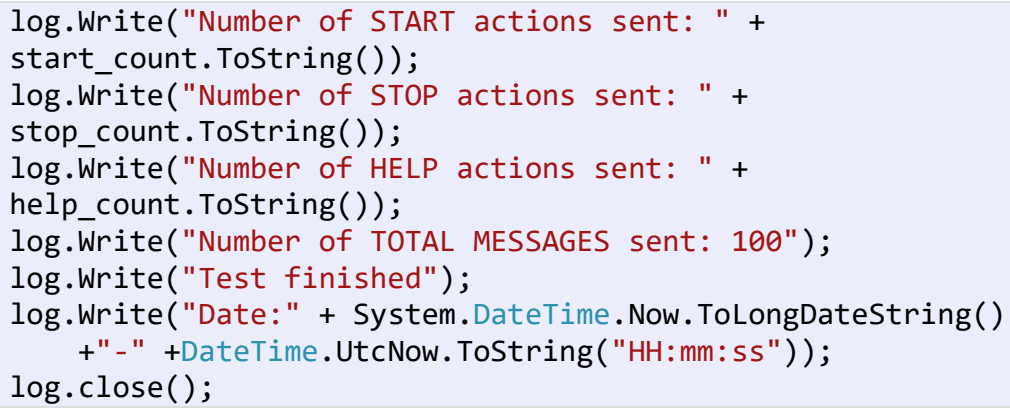

And for the server side:

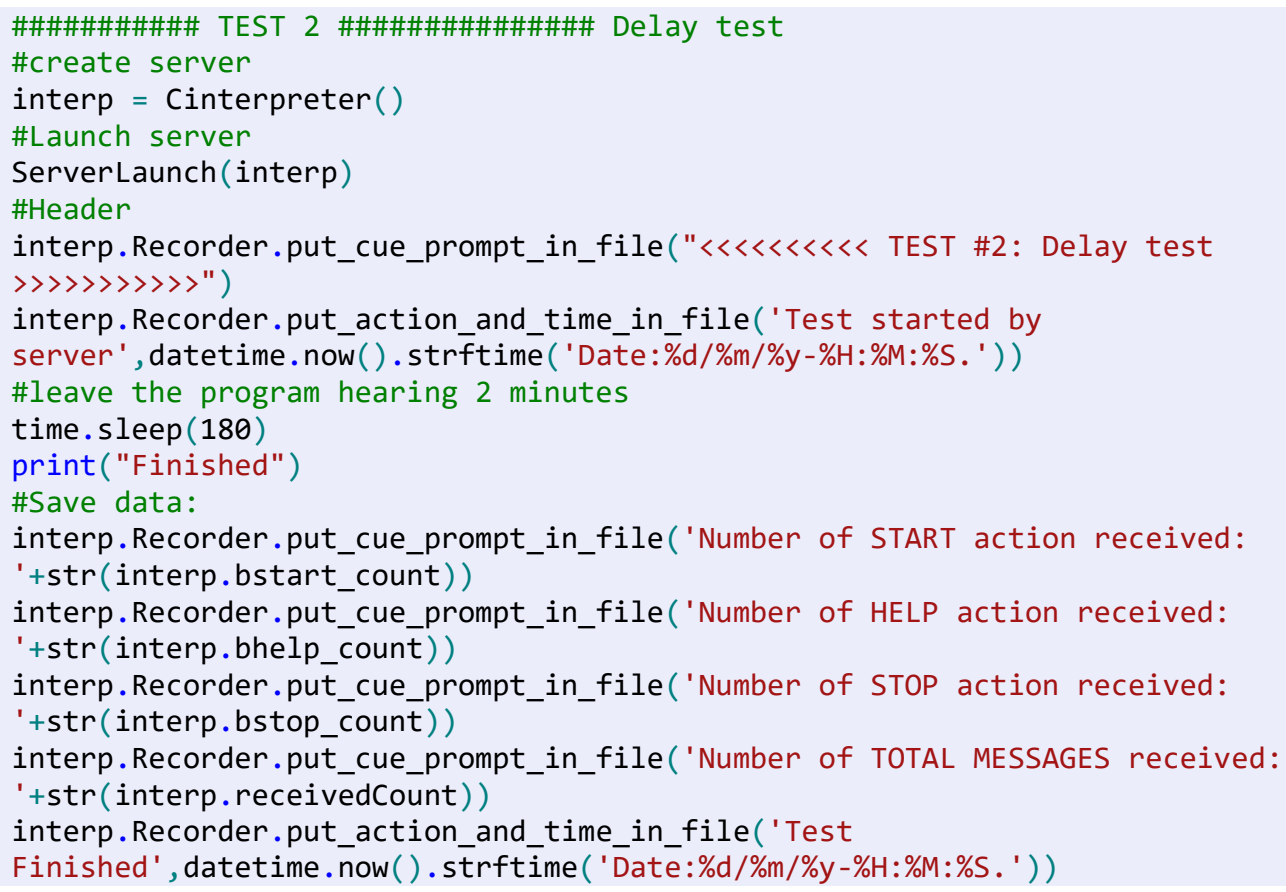

The average delay obtained is $1.03 \mathrm{~ms}$ and the standard deviation is $5.56 \mathrm{~ms}$ the major delay is given by the connection. The following table, Table 19, resumes the delays for each connection expressed in milliseconds: 
Table 19. Test Results for timing test channel 1.

\begin{tabular}{|c|c|c|c|}
\hline Message & Delay [ms] & Message & Delay [ms] \\
\hline$\# 1$ & 31 & $\# 16$ & Less than 1 (N/A) \\
\hline$\# 2$ & Less than 1 (N/A) & $\# 17$ & Less than 1 (N/A) \\
\hline$\# 3$ & Less than 1 (N/A) & $\# 18$ & Less than 1 (N/A) \\
\hline$\# 4$ & Less than 1 (N/A) & $\# 19$ & Less than 1 (N/A) \\
\hline$\# 5$ & Less than 1 (N/A) & $\# 20$ & Less than 1 (N/A) \\
\hline$\# 6$ & Less than 1 (N/A) & $\# 21$ & Less than 1 (N/A) \\
\hline$\# 7$ & Less than 1 (N/A) & $\# 22$ & Less than 1 (N/A) \\
\hline$\# 8$ & Less than 1 (N/A) & $\# 23$ & Less than 1 (N/A) \\
\hline$\# 9$ & Less than 1 (N/A) & $\# 24$ & Less than 1 (N/A) \\
\hline$\# 10$ & Less than 1 (N/A) & $\# 25$ & Less than 1 (N/A) \\
\hline$\# 11$ & Less than 1 (N/A) & $\# 26$ & Less than 1 (N/A) \\
\hline$\# 12$ & Less than 1 (N/A) & $\# 27$ & Less than 1 (N/A) \\
\hline$\# 13$ & Less than 1 (N/A) & $\# 28$ & Less than 1 (N/A) \\
\hline$\# 14$ & Less than 1 (N/A) & $\# 29$ & Less than 1 (N/A) \\
\hline$\# 15$ & Less than 1 (N/A) & $\# 30$ & Less than 1 (N/A) \\
\hline
\end{tabular}

The delays obtained in this test ensure that the socket connection is valid for the application in terms of delays introduced in the system. 


\section{Results from Task Module to Information Handler}

The following code for doing the stress test were used

For the client side:

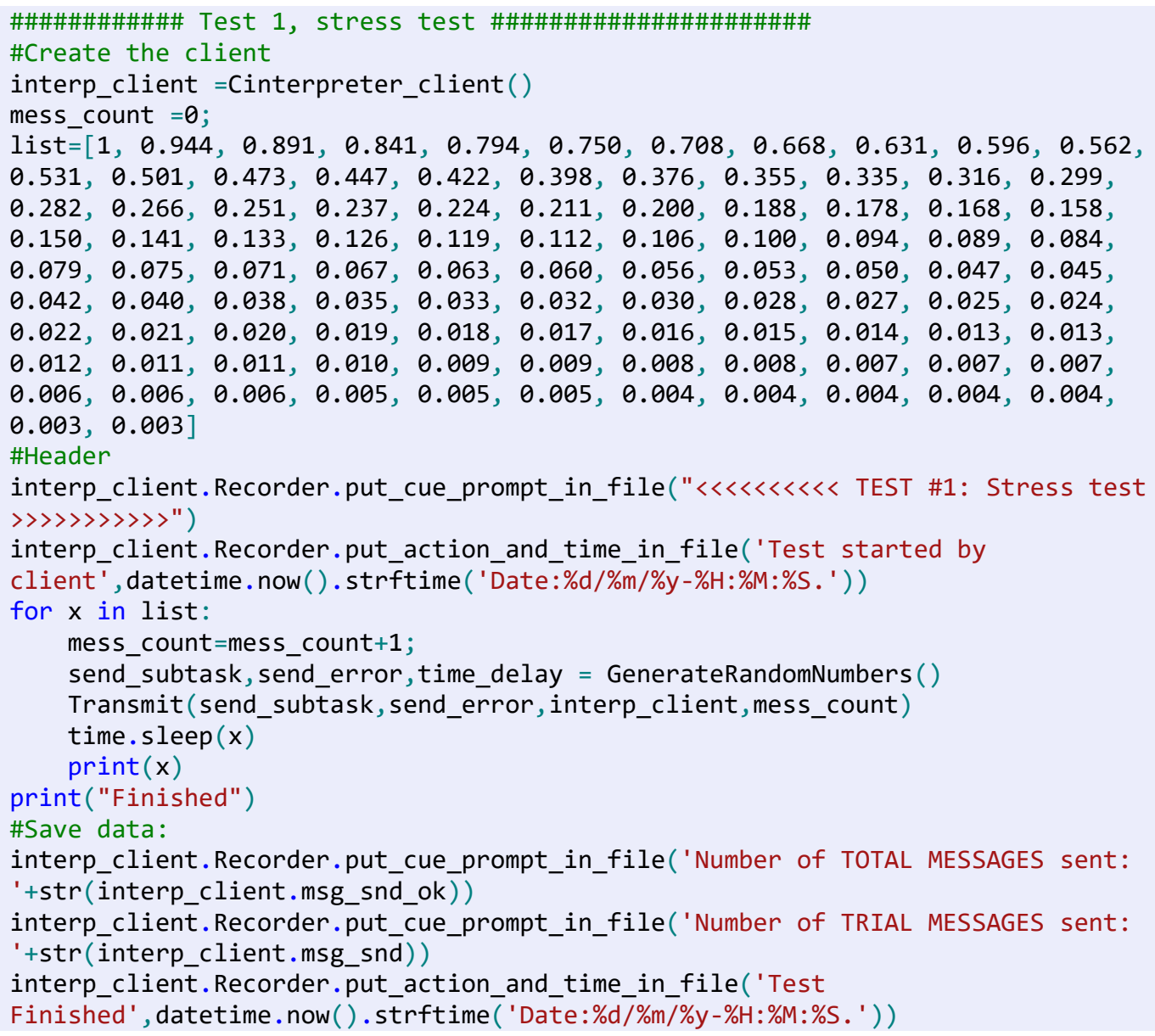

For the server side:

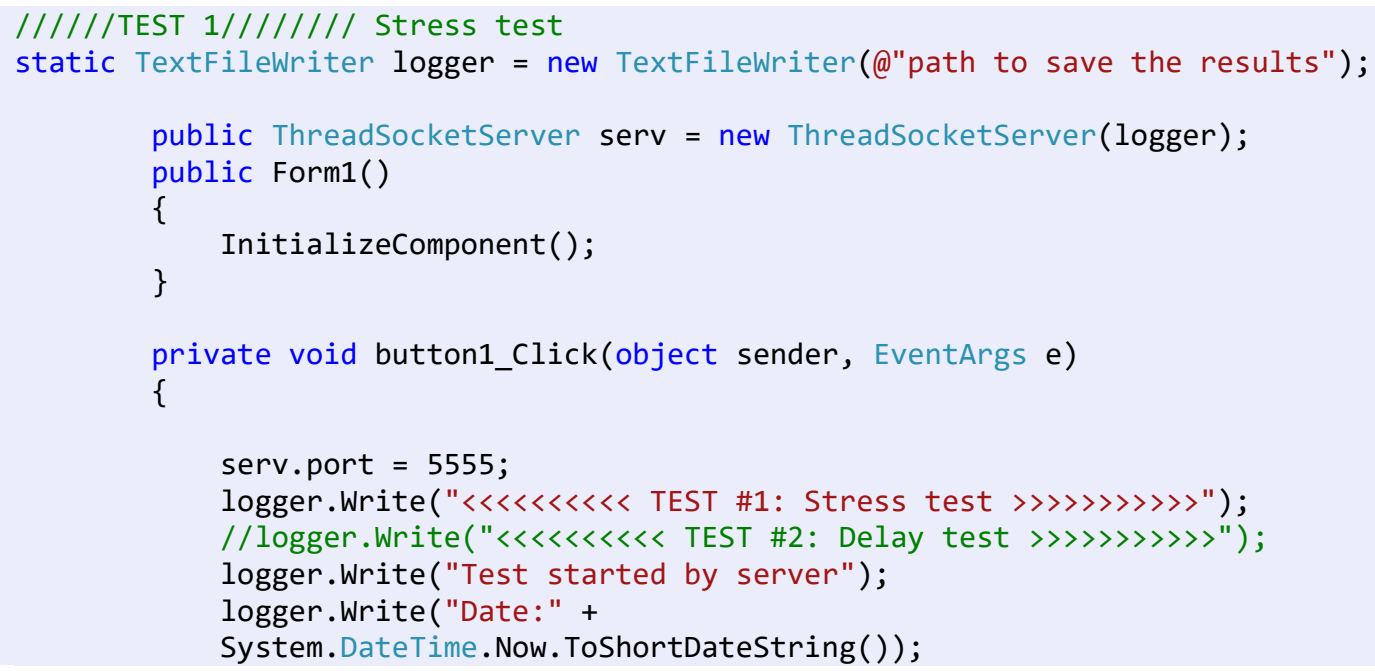




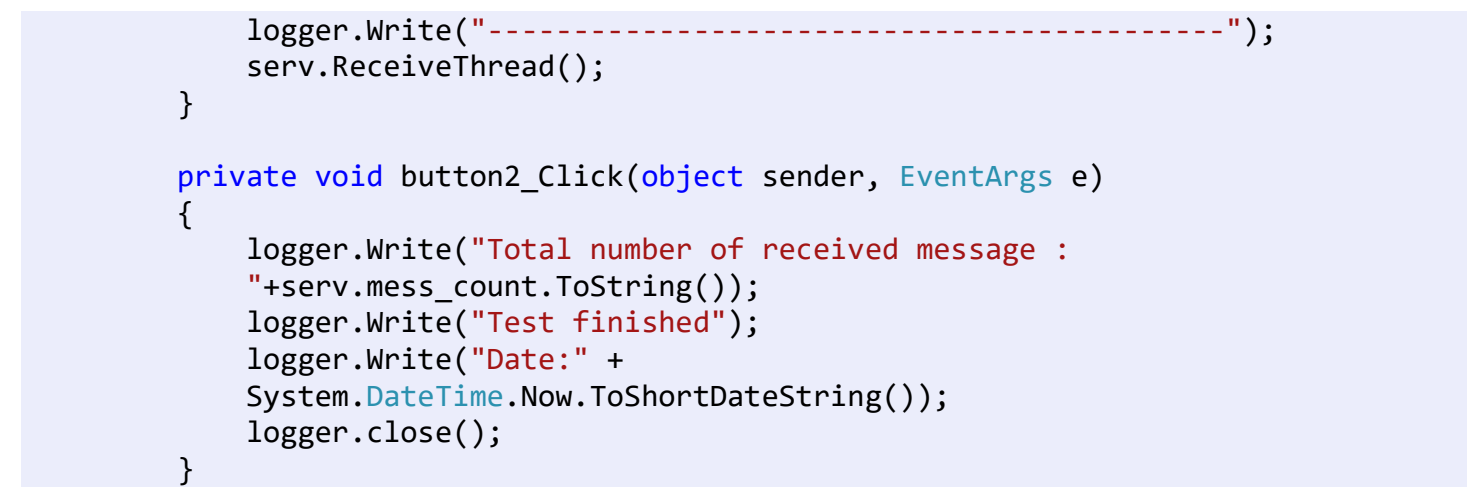

Stress testing results were success. Hundred percent of message sent from client were received from the server and the client does not require to re-sent any message for ensure the correct reception of the message. This is due to the smaller size of the message in comparison with the message exchanged in channel 1 .

The following code for doing the timming test were used

For the client side:

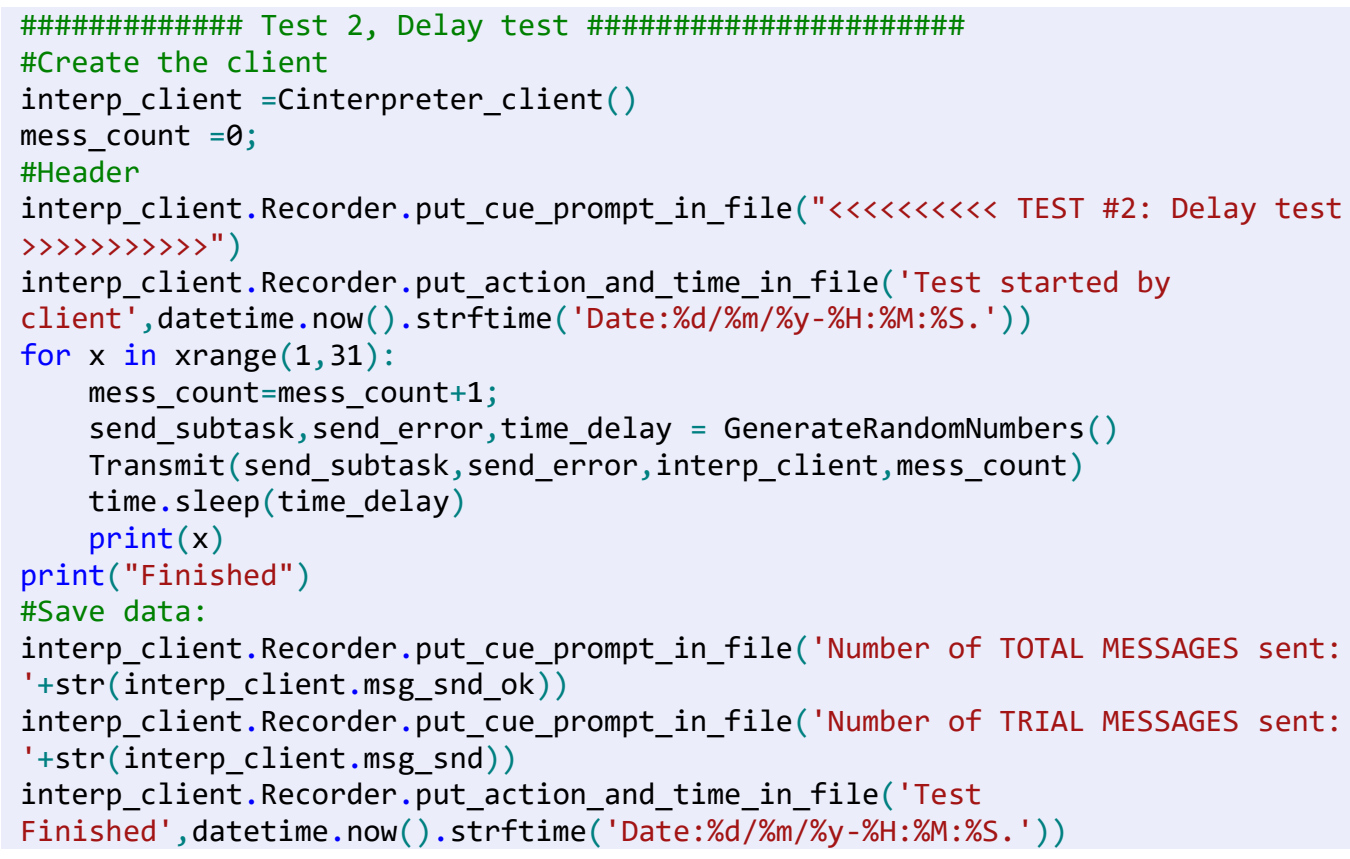


And for the server side:

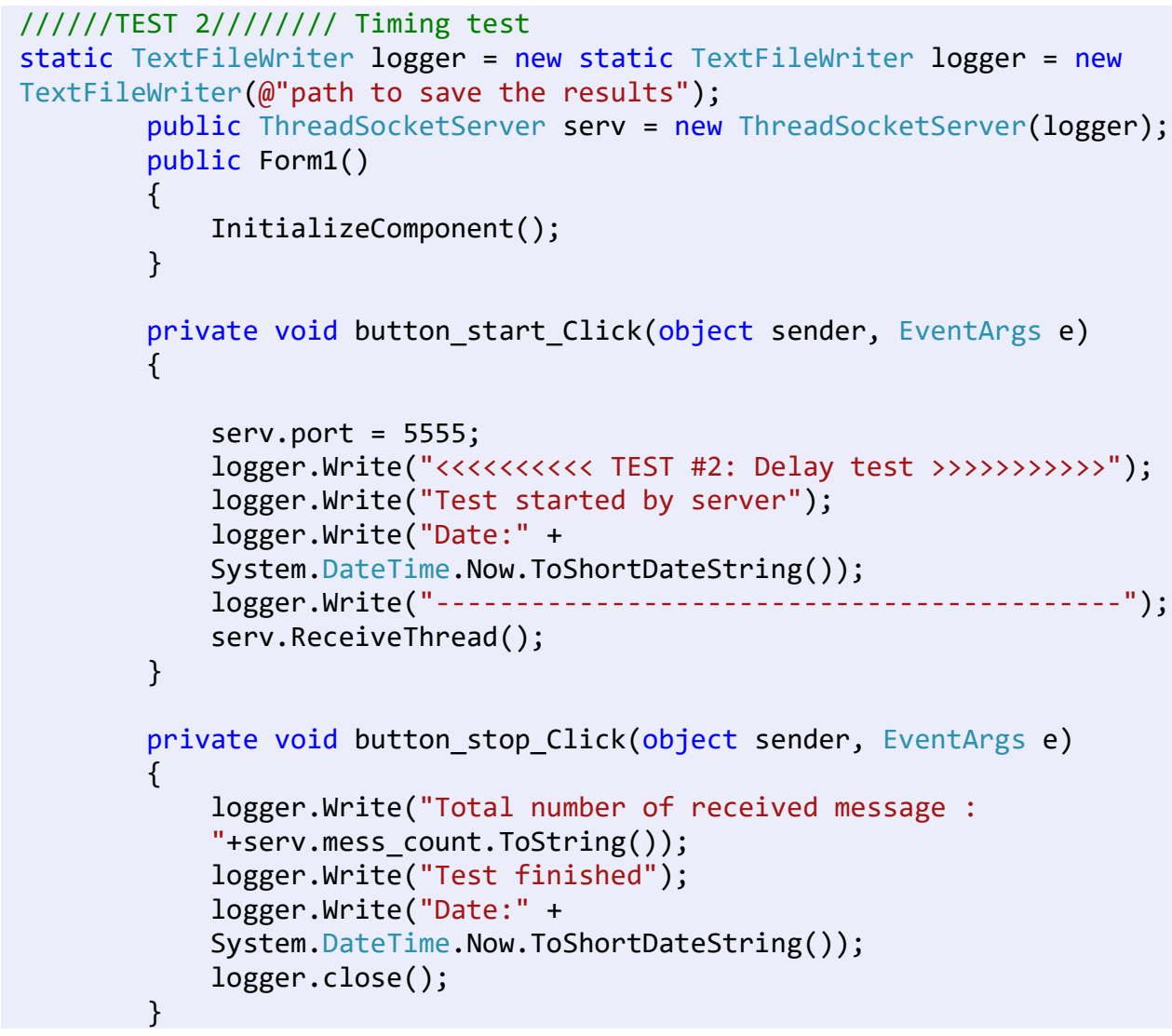

The mean delay obtained is $12.54 \mathrm{~ms}$ and the standard deviation is 22.72 the major delay is given by the connection but in this channel other delays appear. The following table,

Table 20, resumes the delays for each connection expressed in milliseconds:

Table 20.Test Results for timing test channel 2

\begin{tabular}{|c|ccc|}
\hline Message & Delay [ms] & Message & Delay [ms] \\
\hline$\# 1$ & 110 & $\# 16$ & 15 \\
\hline$\# 2$ & Less than 1 (N/A) & $\# 17$ & Less than 1 (N/A) \\
\hline$\# 3$ & Less than 1 (N/A) & $\# 18$ & 31 \\
$\# 4$ & Less than 1 (N/A) & $\# 19$ & Less than 1 (N/A) \\
\hline$\# 5$ & Less than 1 (N/A) & $\# 20$ & Less than 1 (N/A) \\
\hline$\# 6$ & Less than 1 (N/A) & $\# 21$ & Less than 1 (N/A) \\
\hline 7 & 31 & $\# 22$ & Less than 1 (N/A) \\
$\# 8$ & 32 & $\# 23$ & Less than 1 (N/A) \\
$\# 9$ & Less than 1 (N/A) & $\# 24$ & Less than 1 (N/A) \\
\hline
\end{tabular}




\begin{tabular}{|c|c|c|c|}
\hline$\# 10$ & 31 & $\# 25$ & 32 \\
\hline$\# 11$ & Less than 1 (N/A) & $\# 26$ & Less than 1 (N/A) \\
\hline$\# 12$ & 31 & $\# 27$ & Less than 1 (N/A) \\
\hline$\# 13$ & Less than 1 (N/A) & $\# 28$ & Less than 1 (N/A) \\
\hline$\# 14$ & Less than 1 (N/A) & $\# 29$ & 31 \\
\hline$\# 15$ & 32 & $\# 30$ & Less than 1 (N/A) \\
\hline
\end{tabular}

The delays obtained in this test means that the socket connection is valid for the application in terms of delays introduced in the system.

\section{Data from the test:}

The data obtained from the communication tests can be found in the following link:

$\underline{\text { Study results link: }}$

https://docs.google.com/document/d/1Gjl3WmvjWOpoc3Oo1BxuJHDEAD7ejfDjgQ96nGkhYY/edit?usp=sharing 


\section{APPENDIX D}

\section{Reaction Time Meter Description}

This appendix details the material used for the RT study and the characteristics of the RTM developed for this thesis.

\section{Material}

For measuring and recording at work the following materials were used:

A record sheet where the volunteer wrote: gender, age, and the sport in which s/he competes, time it takes to compete in the sport, if they are taking medications, if you have consumed some energy drink, if you have consumed some sort substance that can alter the study of RT, number of hours of sleep that makes daily hours of sleep and enjoyed the day where the test was performed. The investigator wrote down: order of registration and reaction times of each of the tests.

Video camera: a video camera (was used a Canon HG10 - AVCHD) to make records and to check measurements after data collection. It is recorded directly on the hard disk with a capacity of $40 \mathrm{~Gb}$ up to 15 hours of video with a resolution of 1920 x 1080 pixels. Its dimensions are $81 \times 75 \times 129 \mathrm{~mm}$. and a weight of 505 grams. To support this we had a camera tripod.

RTM, Figure 45, is a tool for measuring the Elective Manual Reaction Time to light or sound stimulus.

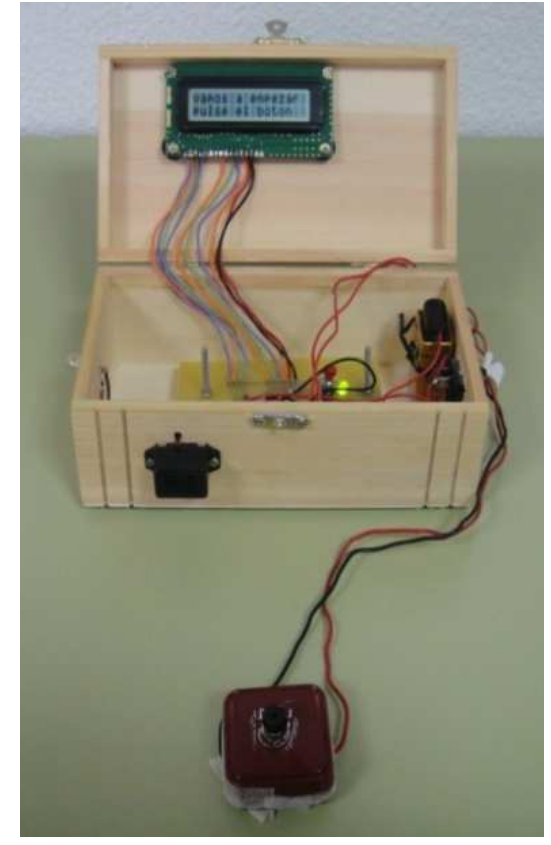

Figure 58. Reaction Time Meter 
The apparatus comprises:

- A switch with a button where the volunteer must press for its RT register.

- On the side, a Light Emitting Diode (LED) arranged in a white color for issuing visual signal.

- On the front, a small speaker for issuing the alarm.

- On the inside, a control panel that lets you apply the operation and start the meter.

- At the top, a recording unit and a Liquid Crystal Display (LCD) reflect reaction times after running visual or sound stimulus.

This apparatus has four sets of random light stimuli and 4 emitted sounds, and a temporary and random interval between them. The technical characteristics of this device are reflected in Appendix D. The accuracy value of RTM is $\pm 0.010 \mathrm{~ms}$.

\section{Working principle}

In this appendix, the characteristics of the instrumentation used for time measurement is described. This machine counts in seconds, with three decimal places, the time elapsed since the device generates a visual or sound stimulus, randomly, until the volunteer activates a button, the process is carried out eight executions in batches interrupting time program until it is re-enabled via pushbutton. The position of the pusher relative to the individual is crucial. In order to accurately evaluate this effect, and hence, and avoid constrains distances are previously measure for each session.

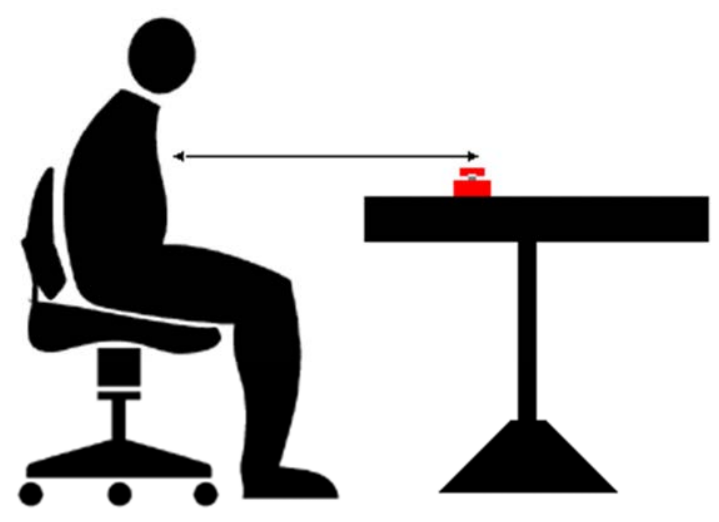

Figure 59. Position of the user and distance reference. 


\section{General schema}

In Figure 60, it is showed the general schema of the circuit.

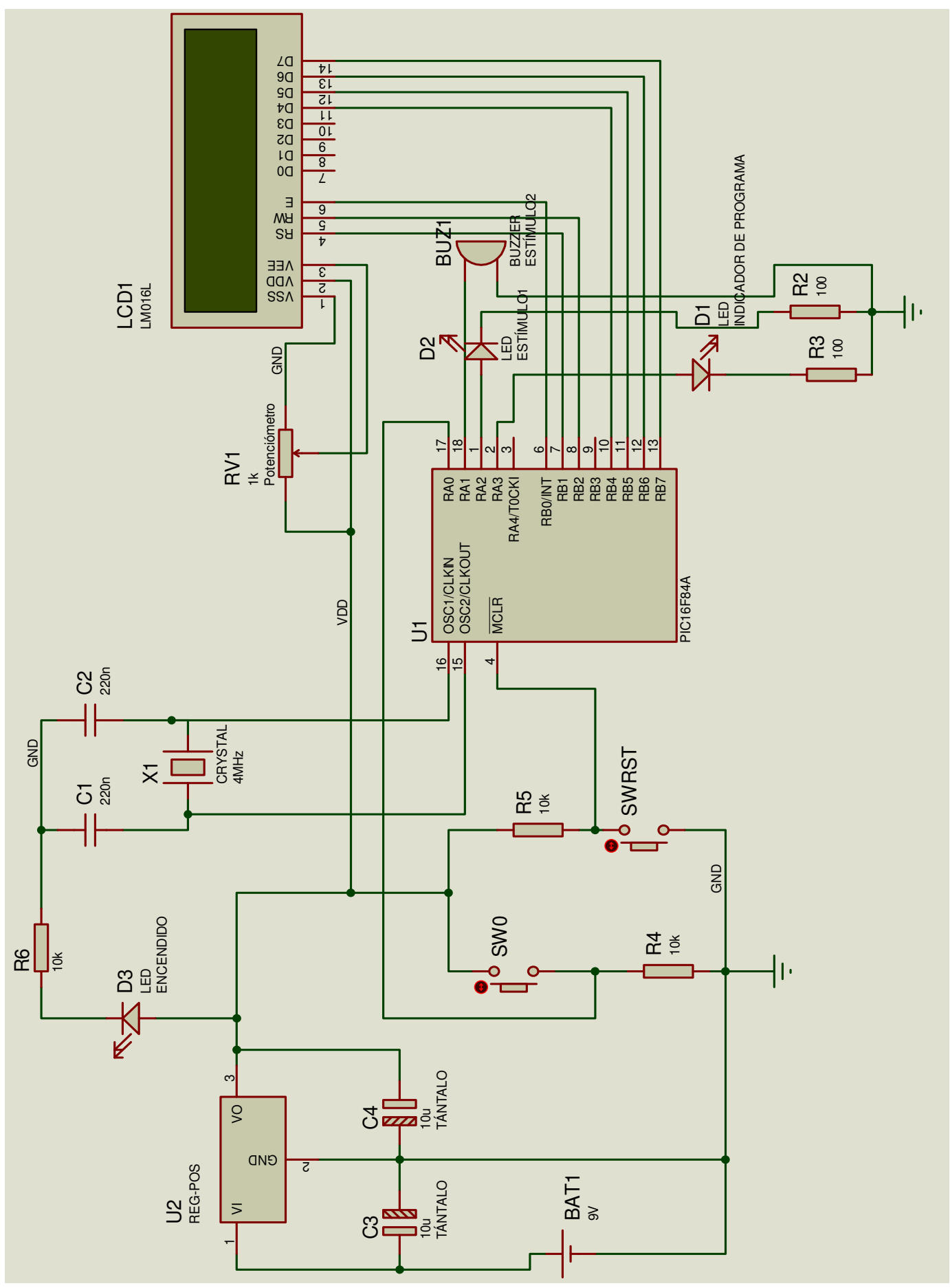

Figure 60. General schema. 


\section{Routing of the circuit}

Once defined the general schema the next step is to route the tracks, i.e. define the physical circuit from a CAD program, in the figure below the layout of the circuit is shown for both sides represented in red, top layer and blue, bottom layer.

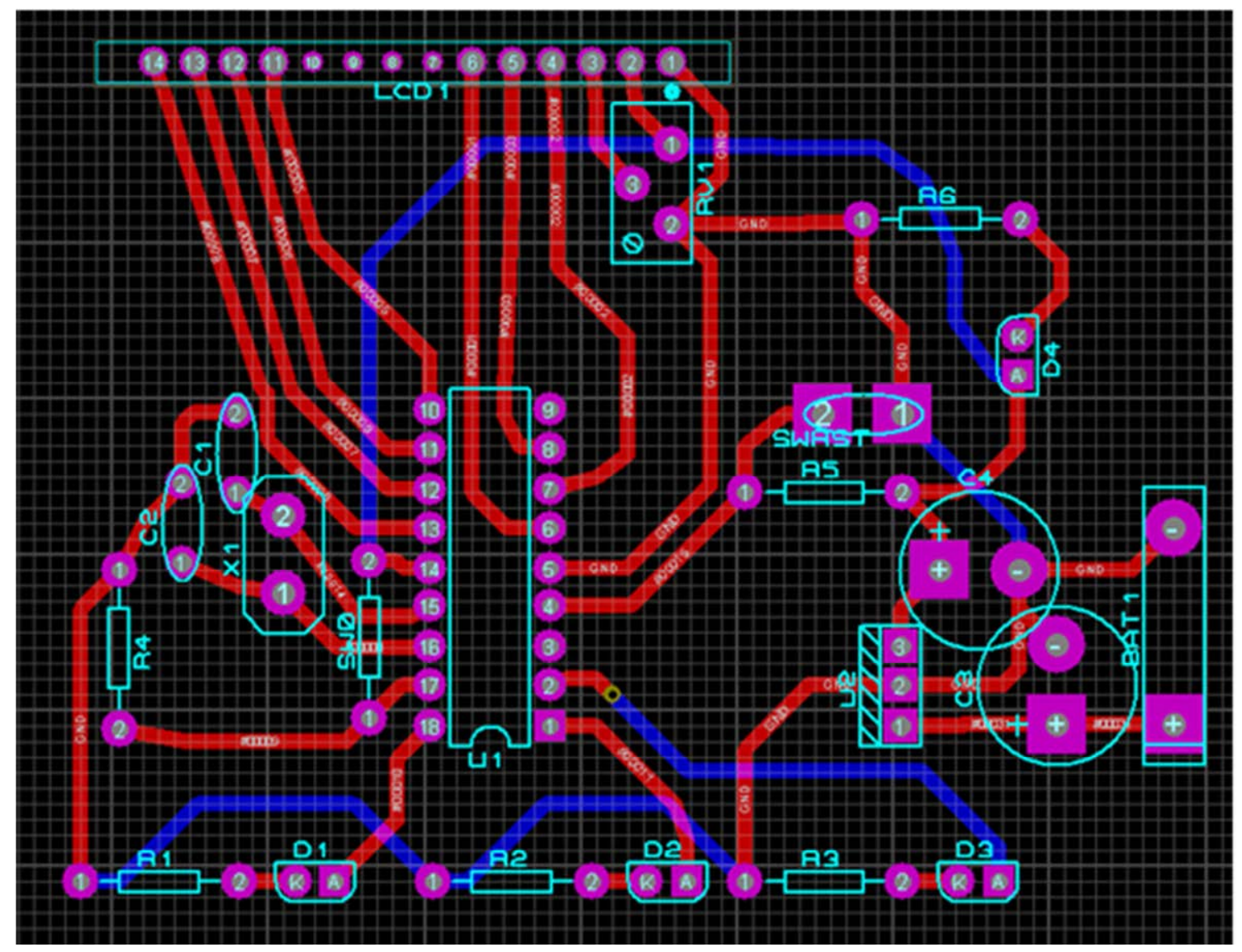

Figure 61. Layout of the circuit. 


\section{ApPendix E}

\section{Non-Invasive Blood Pressure MODUle DESCRIPTION}

The NIBP module, see Figure 62, measures arterial pressure in a NonInvasive way, detecting pressure pulses generated in the air inside the cuff.

Applying this oscillometric method, the following patients' parameters can be derived from detected pulses: systolic, mean and diastolic.

The traditional method for measuring NIBP is inflating the cuff above systolic pressure of the patient and then search by lowering cuff pressure the systolic, mean and diastolic values by oscillometry. This represents a great stress to the patient to bring the cuff pressure to such high values in such a short time. We can say that the measurement method modifies the target parameter to measure (white collar syndrome).

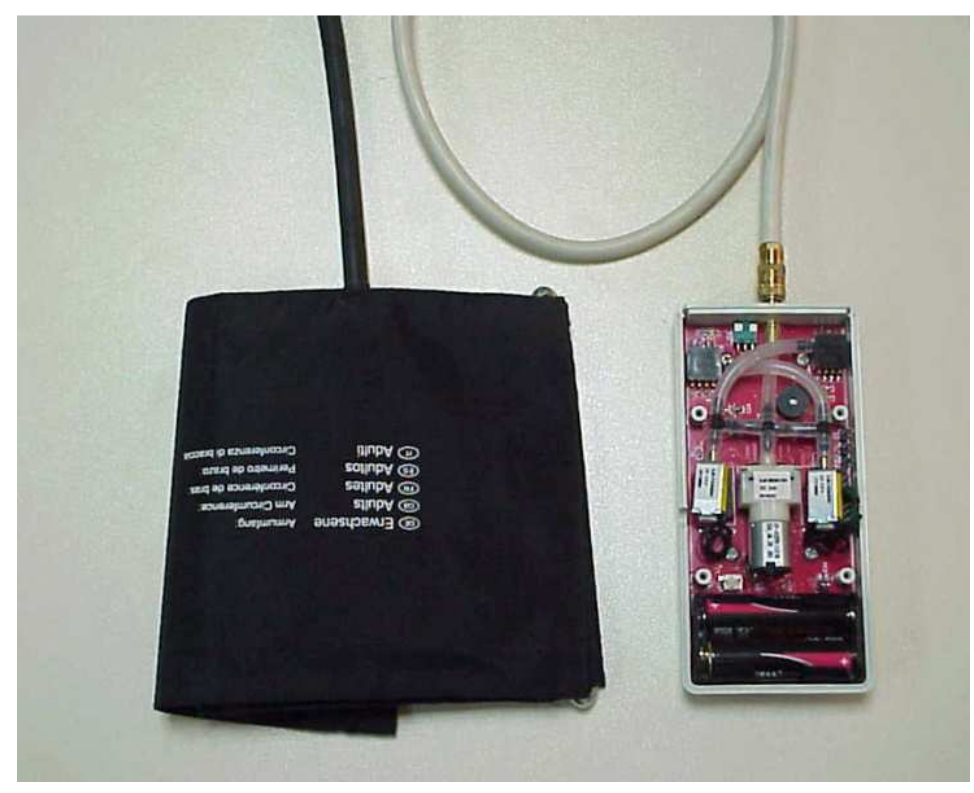

Figure 62. Teardown of NIBP module. 
However, the Cogwatch module has been designed and programmed for measurement during inflation of the cuff, thus measurement is softer and only maintained to a high pressure right at the end of the measurement, and also greatly reducing the measurement time. Therefore, lower stress levels are achieved.

The measurements obtained with this module are systolic, mean and diastolic, and also the HR.

※ Systolic Pressure Range: $35-250 \mathrm{mmHg}$

※ Medium pressure range: $25-234 \mathrm{mmHg}$

※ Diastolic Pressure Range: $20-218 \mathrm{mmHg}$

※ HR Range: 30-250 bpm. 


\section{Appendix F}

\section{ACtion-ERROR DEFINITION TABLES}

This appendix contains the action and error definition tables used for prototypes developed in CogWatch project a summary of the contents is listed below. These tables aim to describe some relevant actions and errors committed by patients of AADS and have been defined by professionals in the matter.

Prototype I: drink preparation task.

※ Actions recognized for tea making.

※ Error definitions for tea making.

Prototype II: grooming task.

※ Actions recognized for tooth brushing.

※ Error definitions for tooth brushing. 


\begin{tabular}{|c|c|c|c|c|c|}
\hline 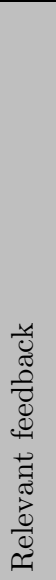 & 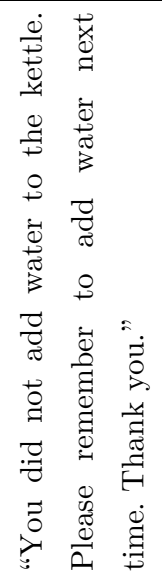 & 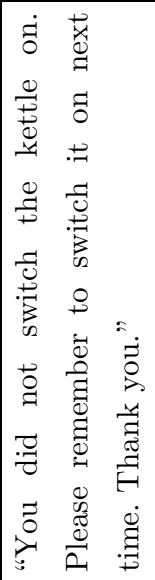 & 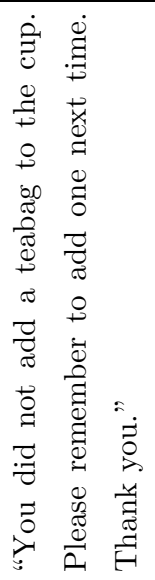 & 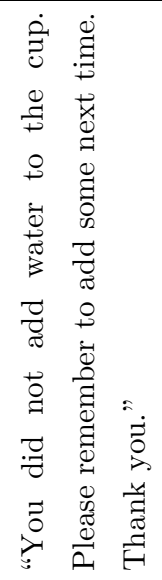 & 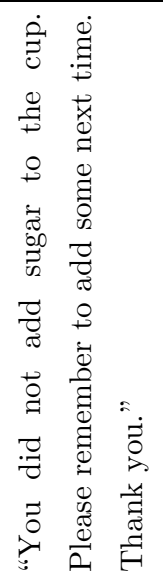 \\
\hline 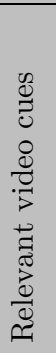 & 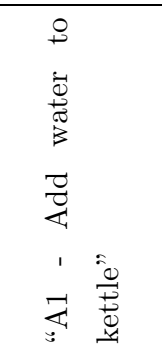 & 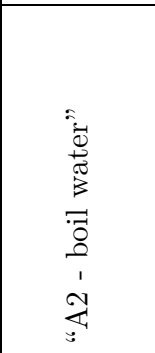 & 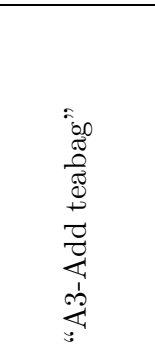 & 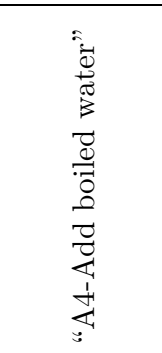 & 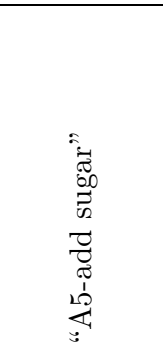 \\
\hline 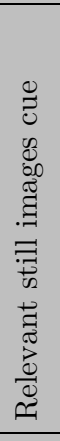 & 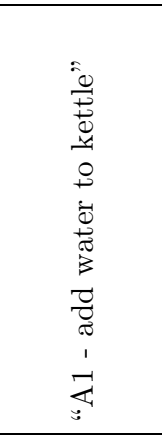 & 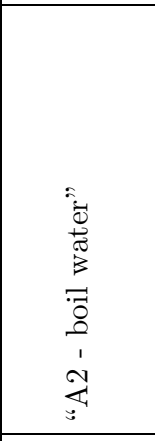 & 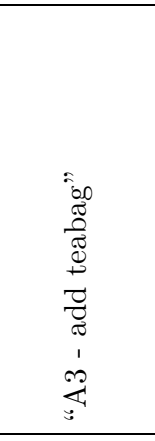 & 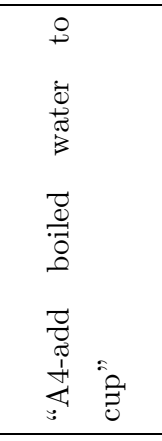 & 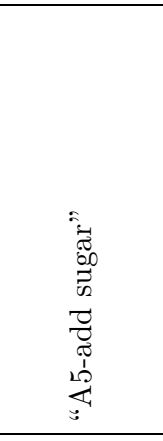 \\
\hline 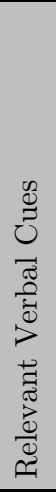 & 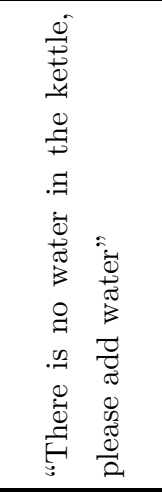 & 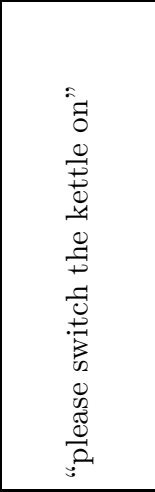 & 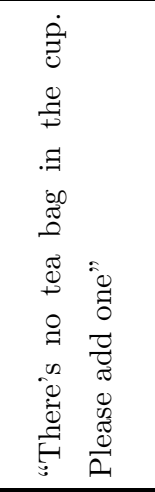 & 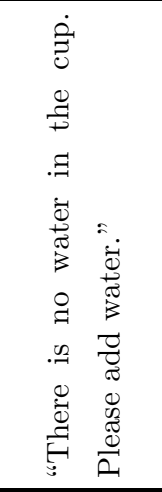 & 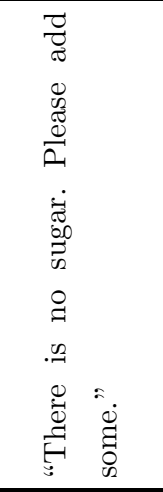 \\
\hline 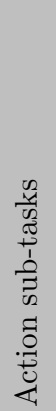 & 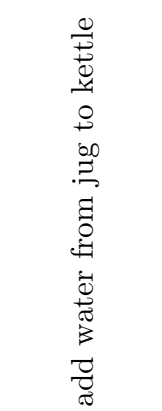 & 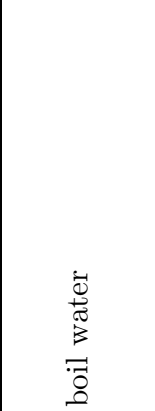 & 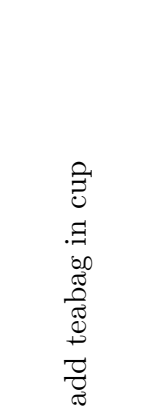 & 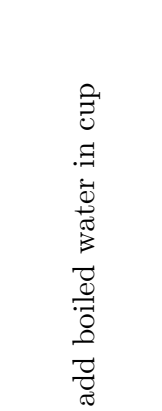 & 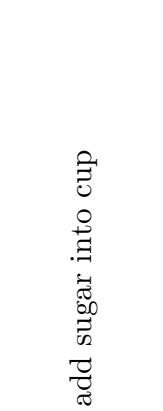 \\
\hline 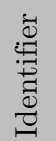 & $\dot{\varangle}$ & ₹ै & 妾 & 䒧 & $\frac{18}{4}$ \\
\hline
\end{tabular}




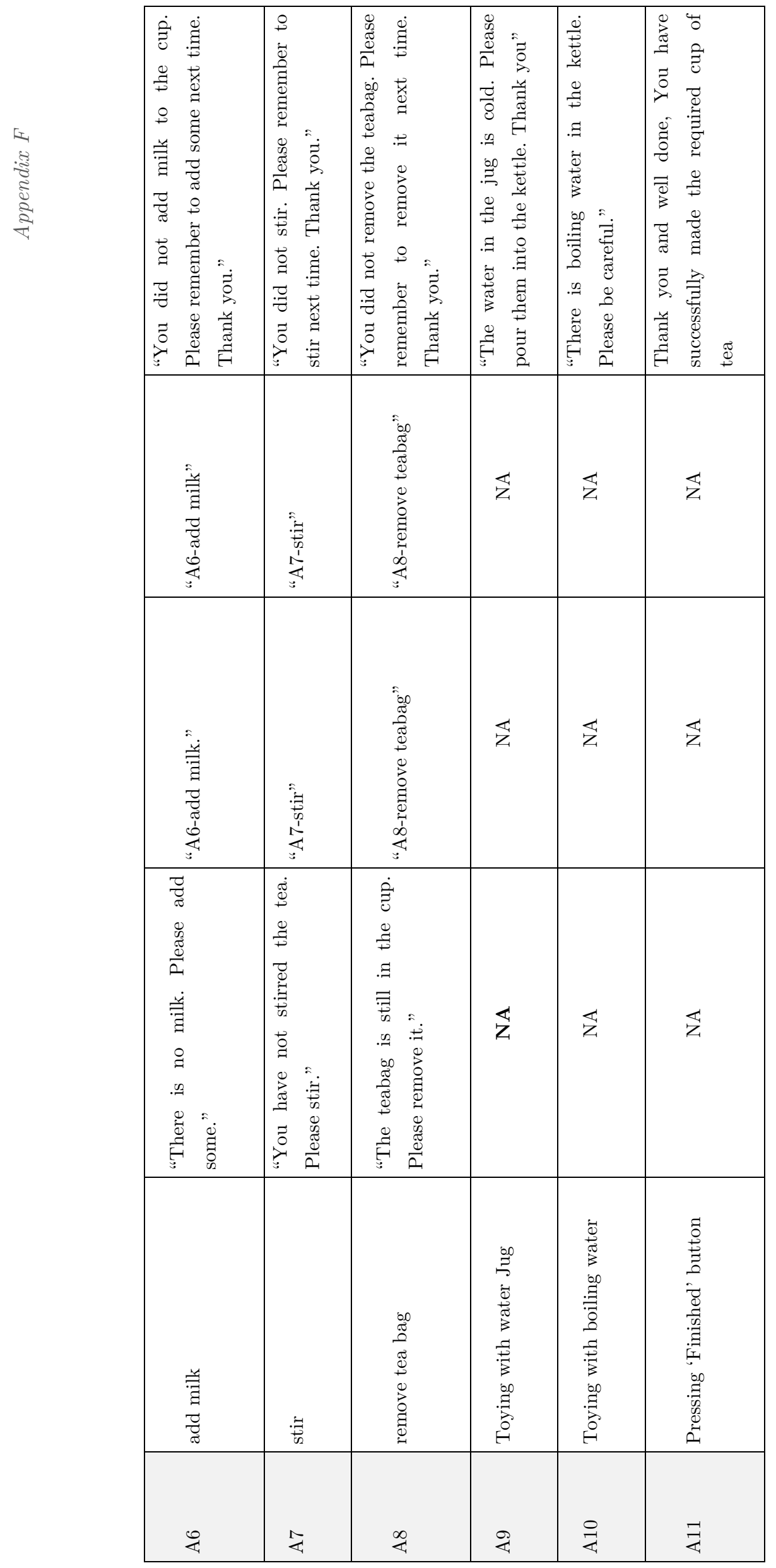




\begin{tabular}{|c|c|c|c|c|c|c|c|}
\hline 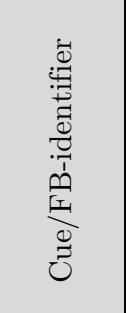 & 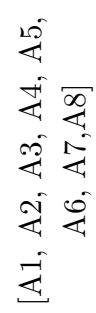 & 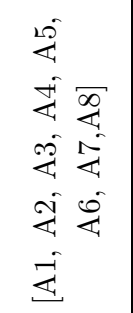 & 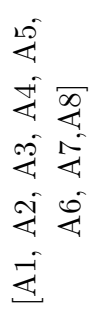 & $\begin{array}{l}\text { 苟 } \\
\text { | } \\
\stackrel{4}{4}\end{array}$ & 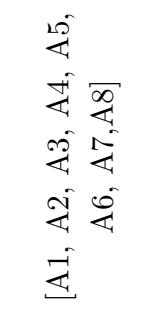 & $\begin{array}{l}0 \\
\& \\
\text { I } \\
\exists \\
\exists\end{array}$ & 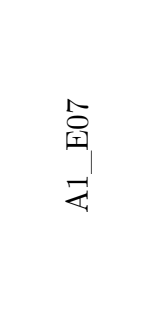 \\
\hline 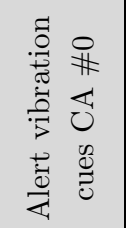 & 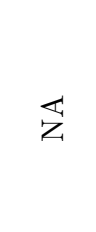 & 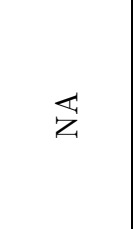 & 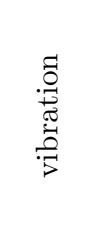 & 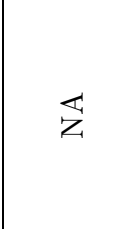 & 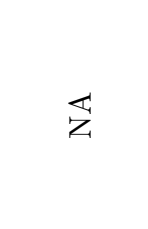 & 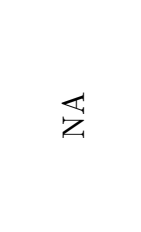 & 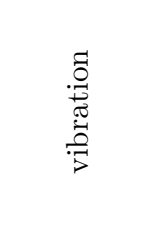 \\
\hline 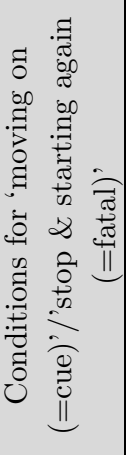 & 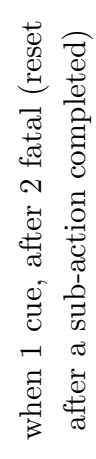 & 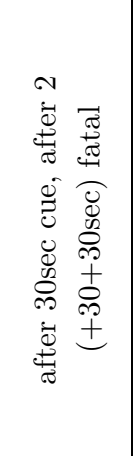 & 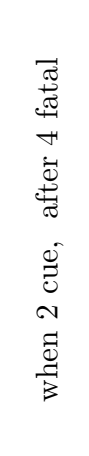 & 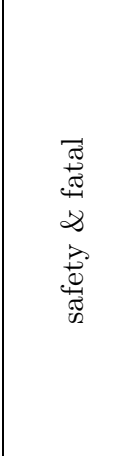 & 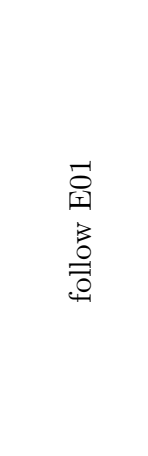 & 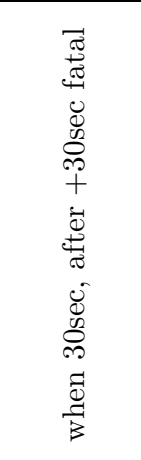 & 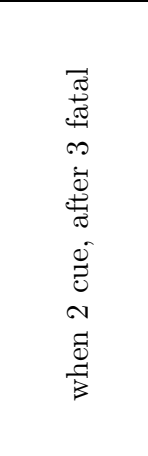 \\
\hline 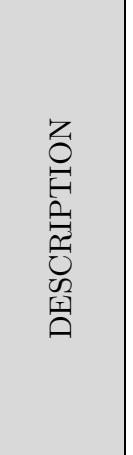 & 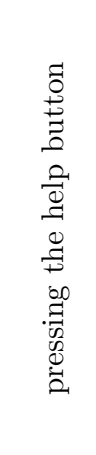 & 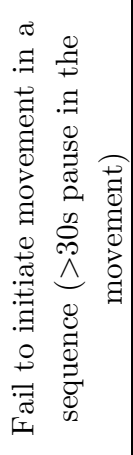 & 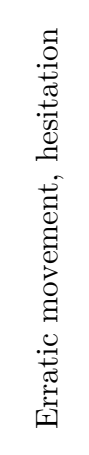 & 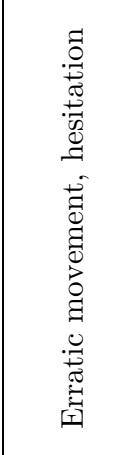 & 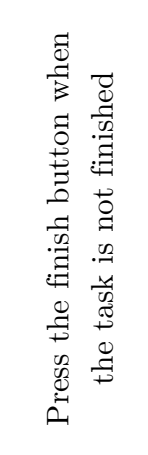 & 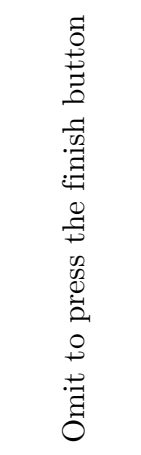 & 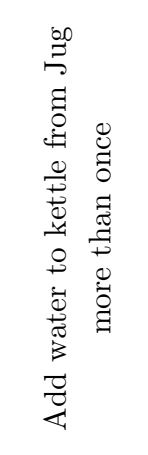 \\
\hline 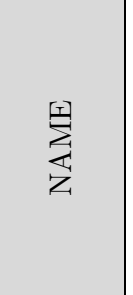 & 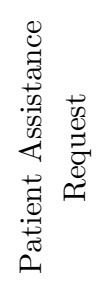 & 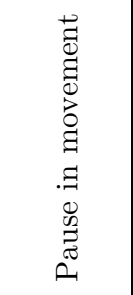 & 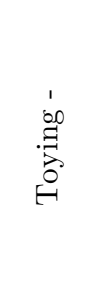 & 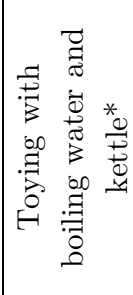 & 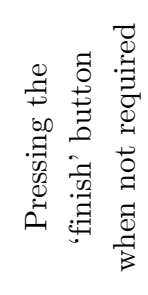 & 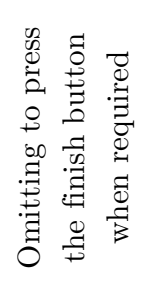 & 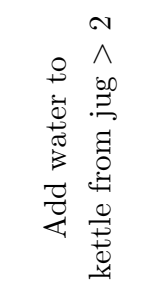 \\
\hline 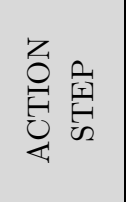 & 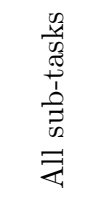 & 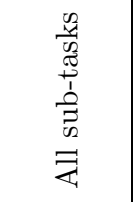 & 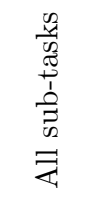 & 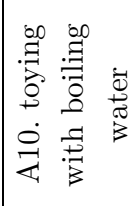 & 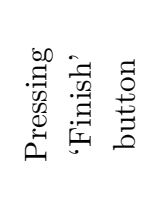 & 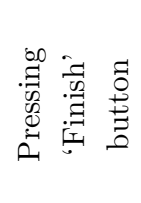 & 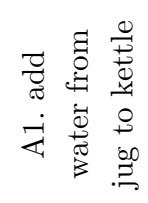 \\
\hline$\sum_{\mathrm{H}}$ & సี & సี & $\bar{\sigma}$ & సี & సี & $\bar{\sigma}$ & $\bar{\sigma}$ \\
\hline Ө & 荃 & $\begin{array}{l}\text { Oे } \\
\text { I. }\end{array}$ & $\begin{array}{l}\text { 胥 } \\
\text {. }\end{array}$ & 葺 & 闰 & \& & 总 \\
\hline
\end{tabular}




\begin{tabular}{|c|c|c|c|c|c|c|c|}
\hline \begin{tabular}{l}
$\infty$ \\
至 \\
$\ddots$ \\
\hdashline
\end{tabular} & $\begin{array}{l}8 \\
\text { 至 } \\
\text { ₹ }\end{array}$ & 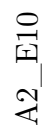 & $\begin{array}{l}\overrightarrow{\vec{r}} \\
\text { 要 }\end{array}$ & 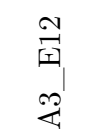 & 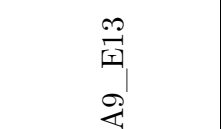 & \begin{tabular}{l}
$\overrightarrow{\mid}$ \\
至 \\
\multirow{2}{*}{}
\end{tabular} & \begin{tabular}{l}
20 \\
至 \\
\multirow{Z}{*}{}
\end{tabular} \\
\hline 広 & $\overleftrightarrow{z}$ & 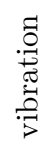 & $\overleftrightarrow{z}$ & 峾 & 吾 & $\overleftrightarrow{z}$ & 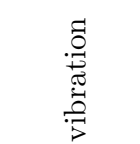 \\
\hline 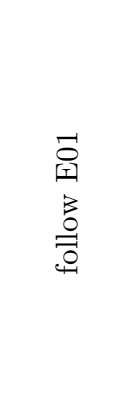 & $\begin{array}{l}\overrightarrow{0} \\
\text { 采 } \\
3 \\
0 \\
0 \\
0 \\
0\end{array}$ & 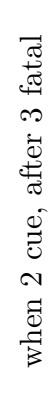 & $\begin{array}{l}\text { 오 } \\
\text { I } \\
3 \\
0 \\
0 \\
0\end{array}$ & 焉 & 亚 & 稳 & 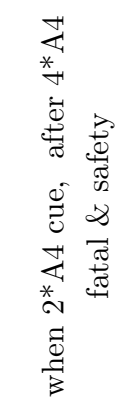 \\
\hline 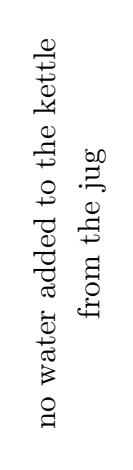 & 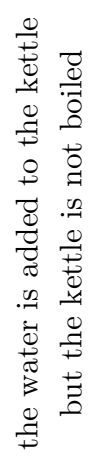 & 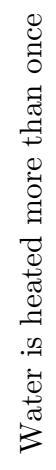 & 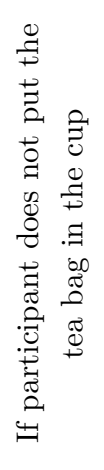 & 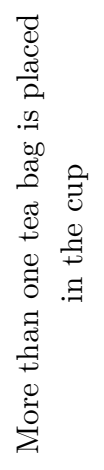 & 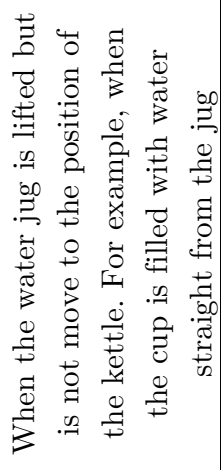 & 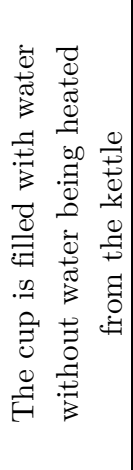 & 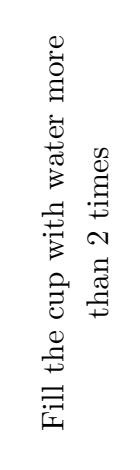 \\
\hline 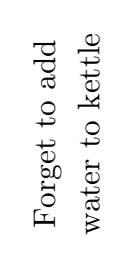 & 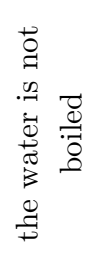 & $\begin{array}{l}\vec{\wedge} \\
\hat{0} \\
\overrightarrow{0} \\
\overrightarrow{3} \\
\overrightarrow{0} \\
\overrightarrow{0}\end{array}$ & 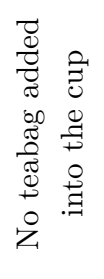 & 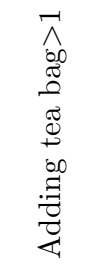 & 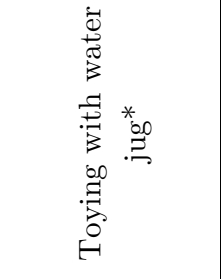 & 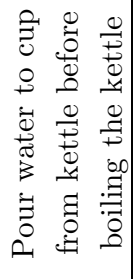 & 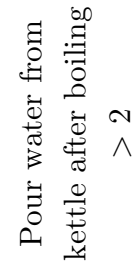 \\
\hline 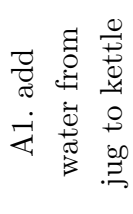 & 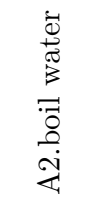 & 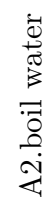 & 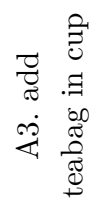 & 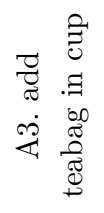 & 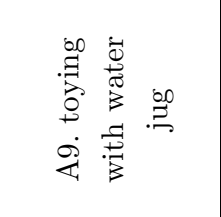 & 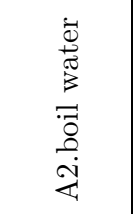 & 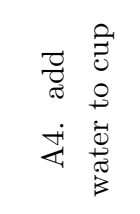 \\
\hline $\bar{\sigma}$ & $\bar{\sigma}$ & $\bar{\sigma}$ & $\bar{\sigma}$ & $\bar{\sigma}$ & సี & $\bar{\nabla}$ & $\bar{\sigma}$ \\
\hline $\begin{array}{l}\infty \\
0 \\
\text { I. }\end{array}$ & 总 & $\overrightarrow{\mid r}$ & $\overrightarrow{\mid r}$ & II & $\frac{9}{4}$ & 堊 & $\frac{12}{19}$ \\
\hline
\end{tabular}




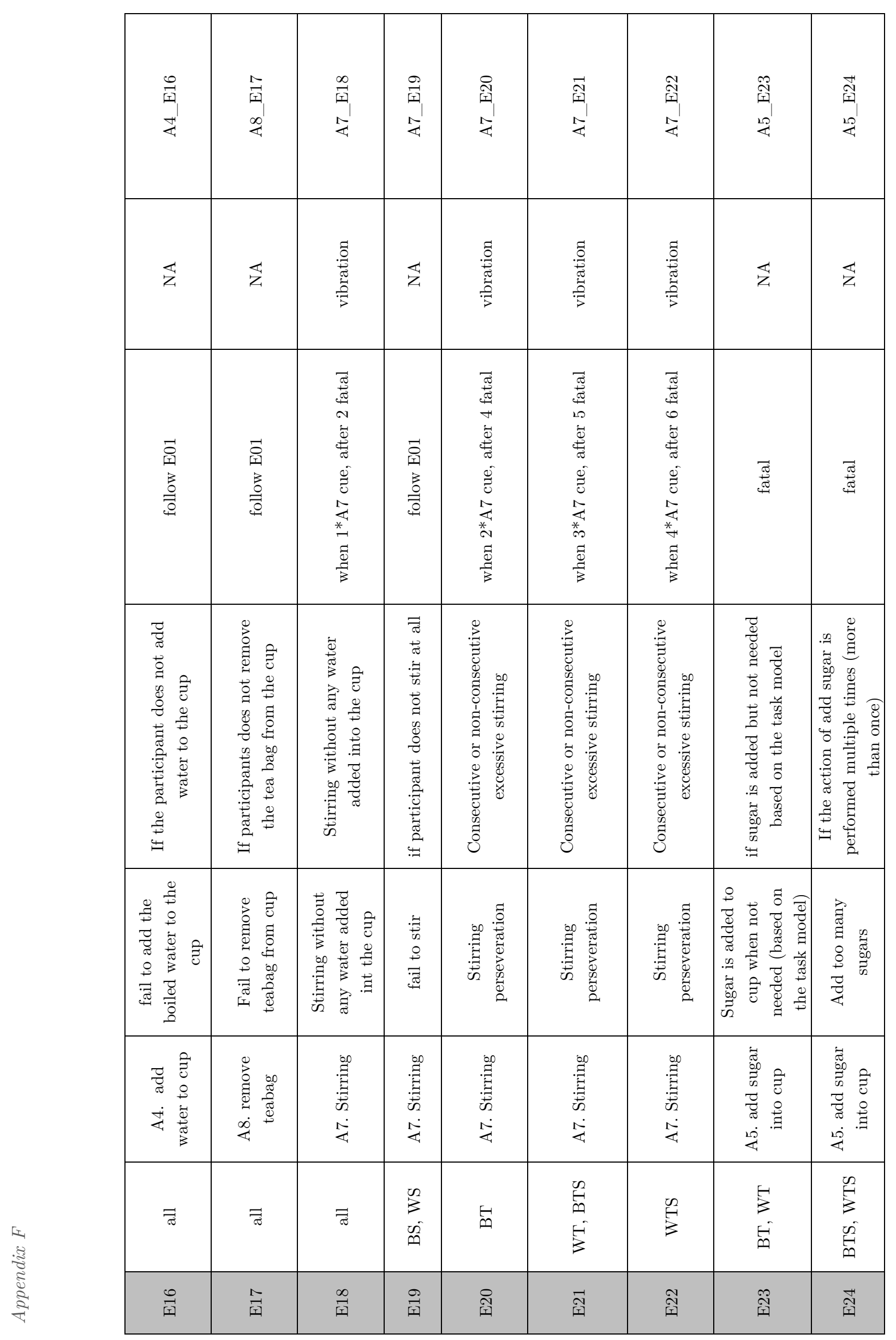




\begin{tabular}{|c|c|c|c|c|c|c|}
\hline 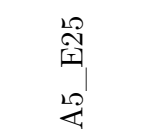 & 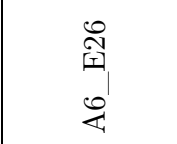 & $\begin{array}{l}\text { Nิ } \\
\text { 表 } \\
0\end{array}$ & $\begin{array}{l}\infty \\
\text { 空 } \\
0 \\
0\end{array}$ & $\begin{array}{l}\text { 缶 } \\
\text { दै }\end{array}$ & $\begin{array}{l}0 \\
\text { 雳 } \\
\text { 足 }\end{array}$ & $\vec{U}$ \\
\hline 艺 & 艺 & 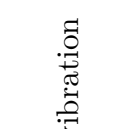 & 艺 & 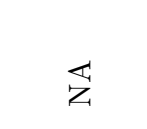 & $\overleftrightarrow{\mathrm{z}}$ & \\
\hline 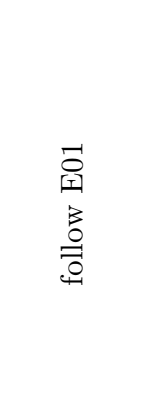 & 胥 & 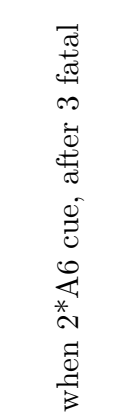 & 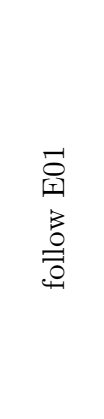 & $\overleftrightarrow{z}$ & 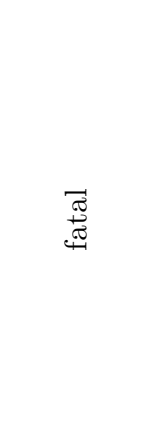 & \\
\hline 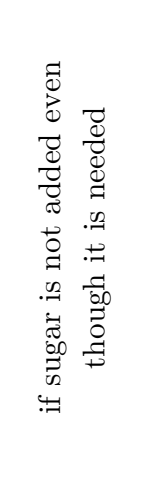 & 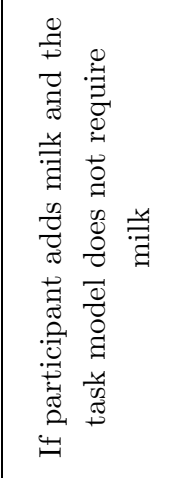 & 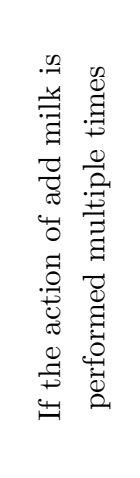 & 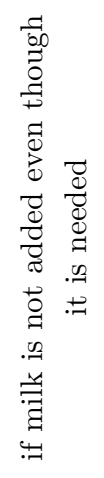 & 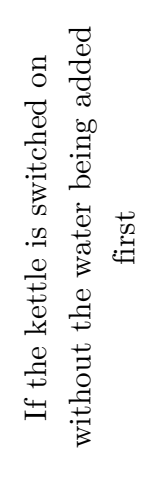 & 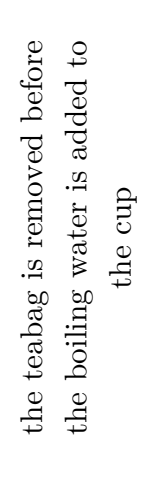 & 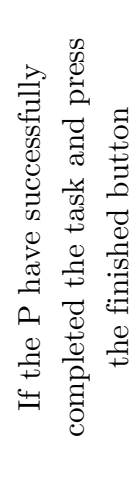 \\
\hline 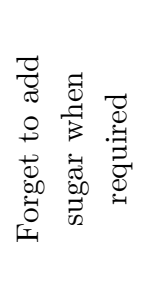 & 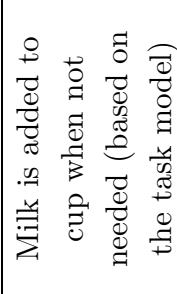 & 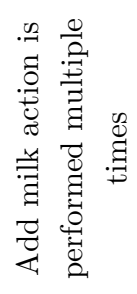 & 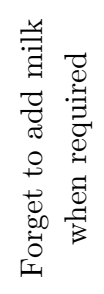 & 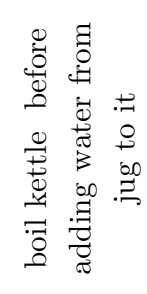 & 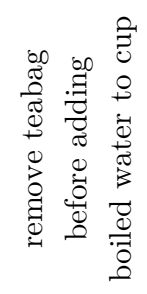 & 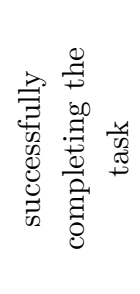 \\
\hline 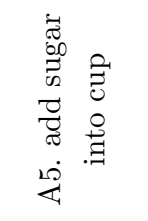 & 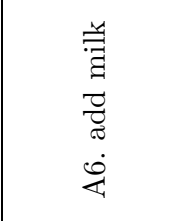 & 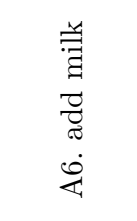 & 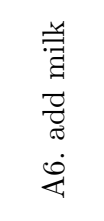 & 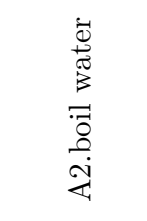 & 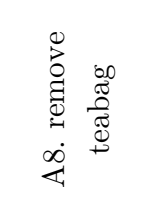 & 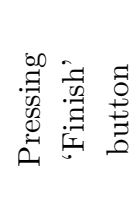 \\
\hline 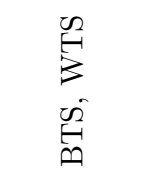 & 点 & $\begin{array}{l}02 \\
5 \\
3 \\
5-1 \\
3\end{array}$ & $\begin{array}{l}2 \\
5 \\
3 \\
5 \\
3 \\
3\end{array}$ & $\bar{\tau}$ & $\overline{\widetilde{\sigma}}$ & శี \\
\hline $\begin{array}{l}\stackrel{2}{N} \\
\text { 竍 }\end{array}$ & స్ & $\begin{array}{l}\hat{N} \\
\text { స్ }\end{array}$ & 㒰 & 胥 & 炁 & $\overrightarrow{8}$ \\
\hline
\end{tabular}




\begin{tabular}{|c|c|c|c|c|c|c|c|}
\hline 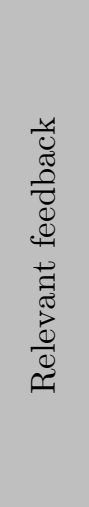 & 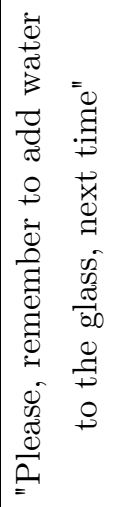 & 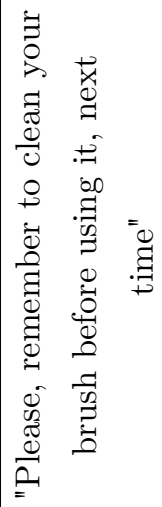 & 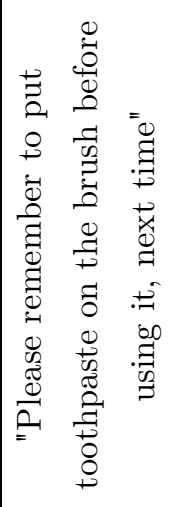 & 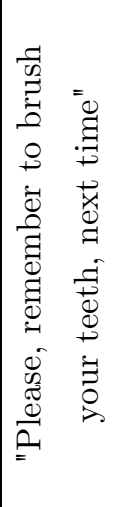 & 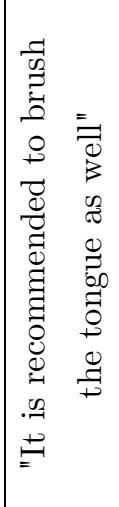 & 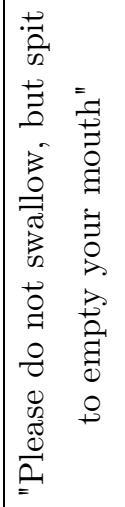 & 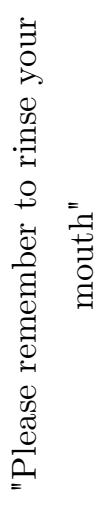 \\
\hline 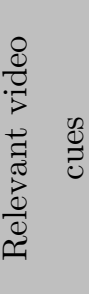 & 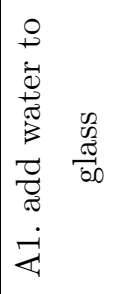 & 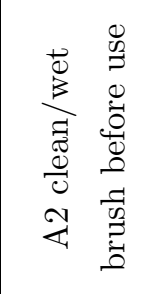 & 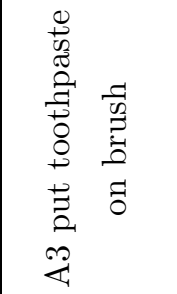 & 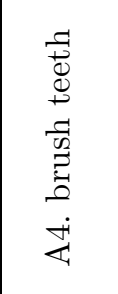 & $\begin{array}{l}0 \\
50 \\
5 \\
0 \\
0 \\
5 \\
5 \\
5 \\
0 \\
\dot{0} \\
\dot{0}\end{array}$ & $\begin{array}{l}\overrightarrow{\vec{n}} \\
\text { की } \\
\dot{0}\end{array}$ & 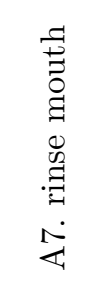 \\
\hline 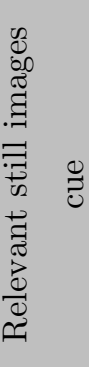 & 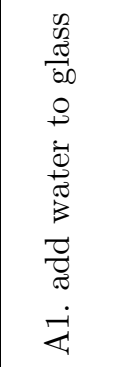 & 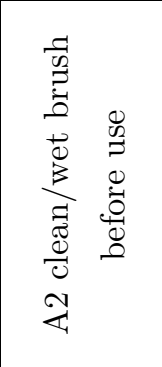 & 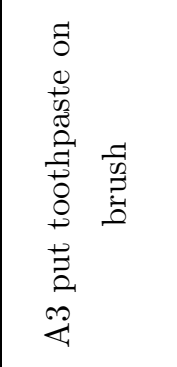 & 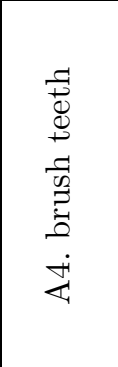 & 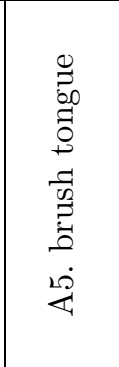 & $\begin{array}{l}\overrightarrow{\vec{n}} \\
\dot{2} \\
\dot{\varphi}\end{array}$ & 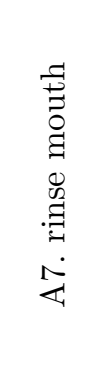 \\
\hline 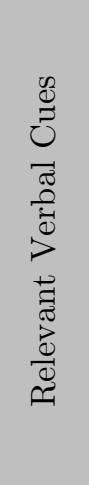 & 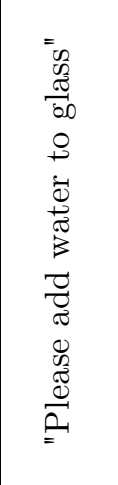 & 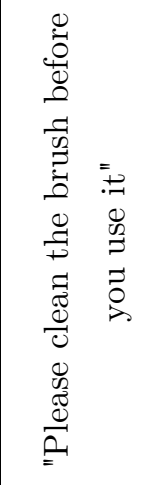 & 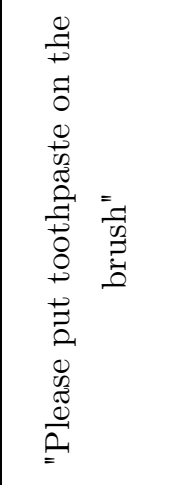 & 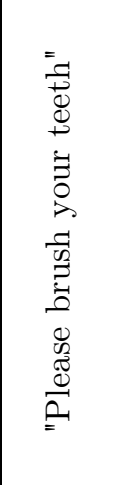 & 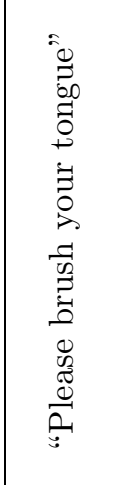 & 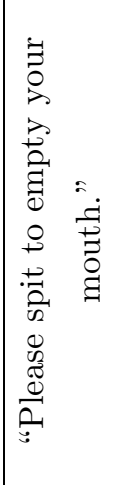 & 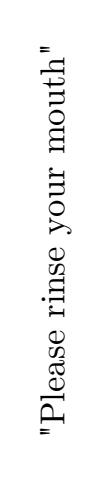 \\
\hline 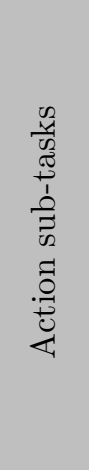 & 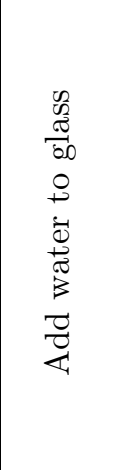 & 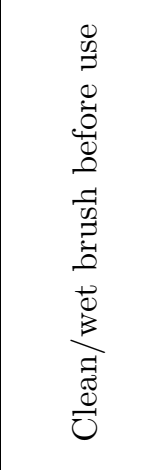 & 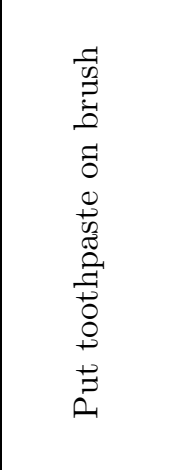 & 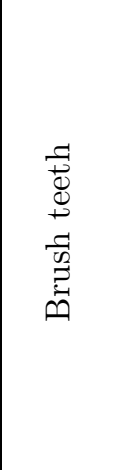 & 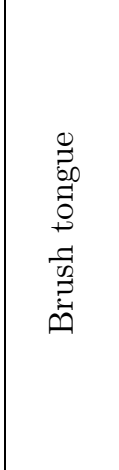 & 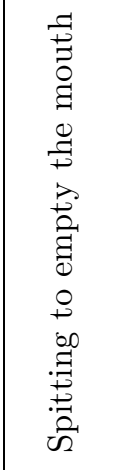 & 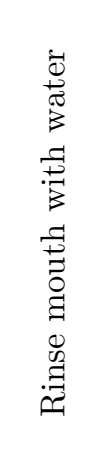 \\
\hline 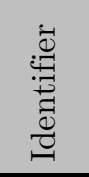 & ¿ & \& & $\stackrel{\infty}{\gtrless}$ & $\underset{4}{4}$ & $\stackrel{10}{4}$ & $\stackrel{0}{4}$ & 年 \\
\hline
\end{tabular}




\begin{tabular}{|c|c|c|c|c|c|c|}
\hline 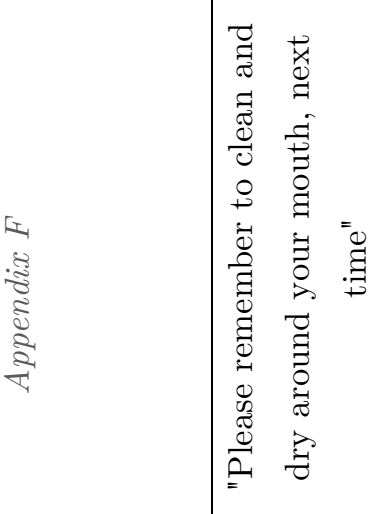 & 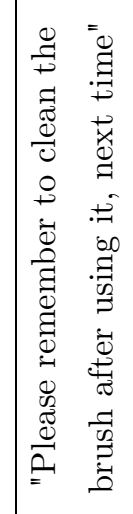 & 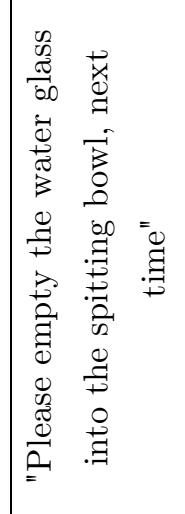 & 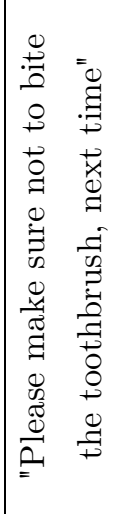 & 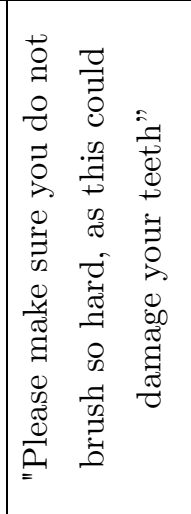 & 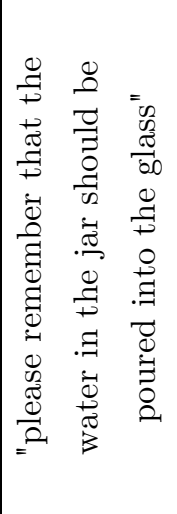 & 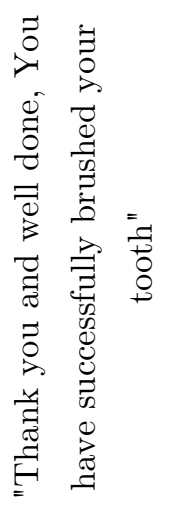 \\
\hline 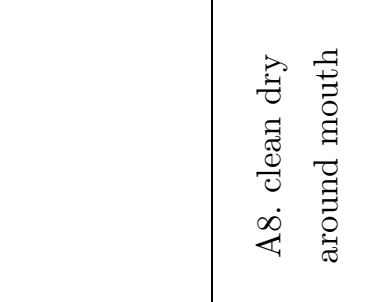 & 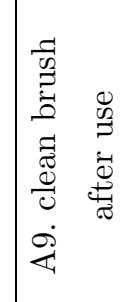 & 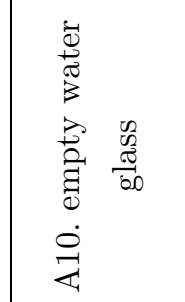 & 艺 & 艺 & 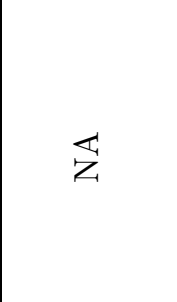 & 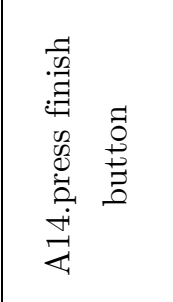 \\
\hline 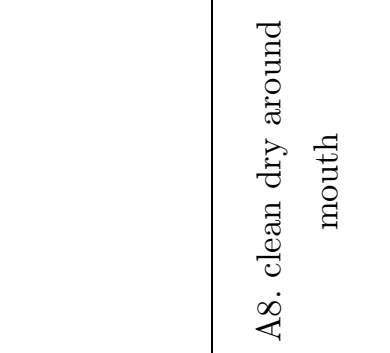 & 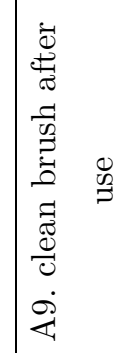 & 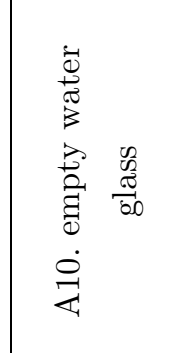 & 艺 & $\overleftrightarrow{Z}$ & 艺 & 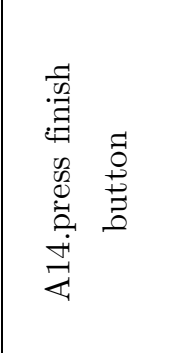 \\
\hline 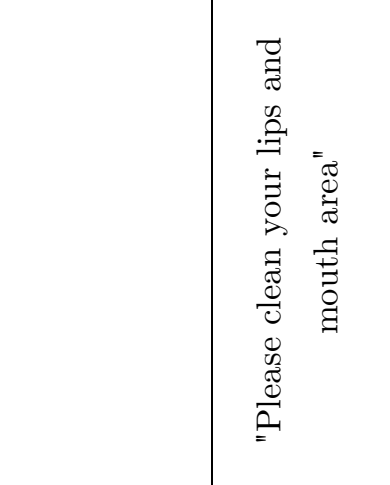 & 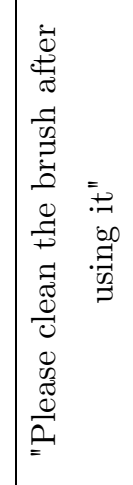 & 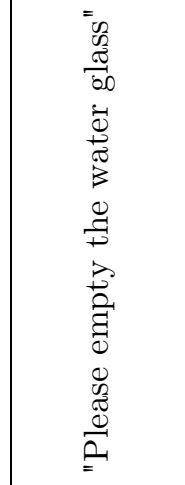 & 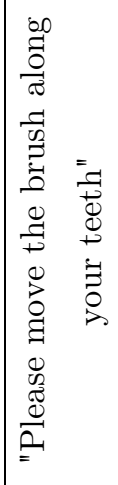 & 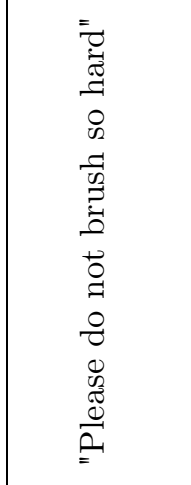 & 死 & \\
\hline 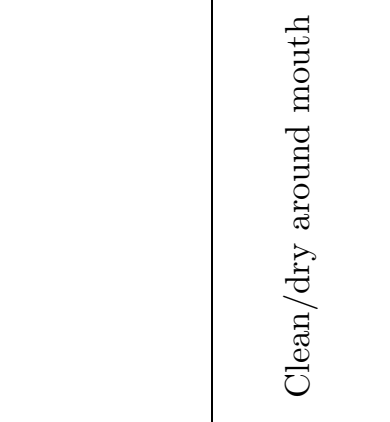 & 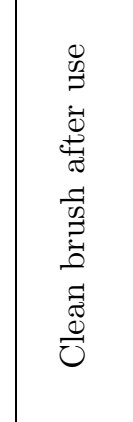 & 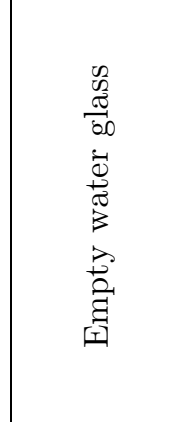 & 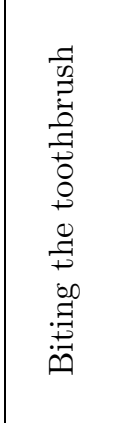 & 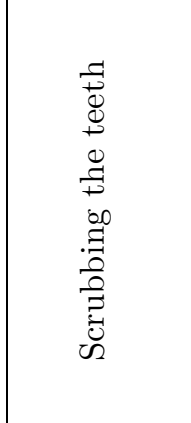 & 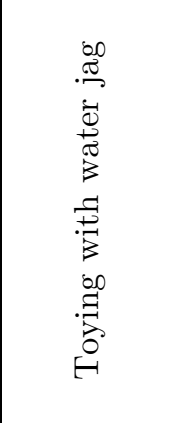 & 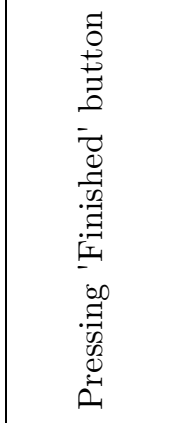 \\
\hline $\mathbb{4}_{4}^{\infty}$ & \& & 疍 & 光 & $\stackrel{\mathcal{Z}}{\mathrm{Z}}$ & $\stackrel{\infty}{\sharp}$ & $\underset{4}{Z}$ \\
\hline
\end{tabular}




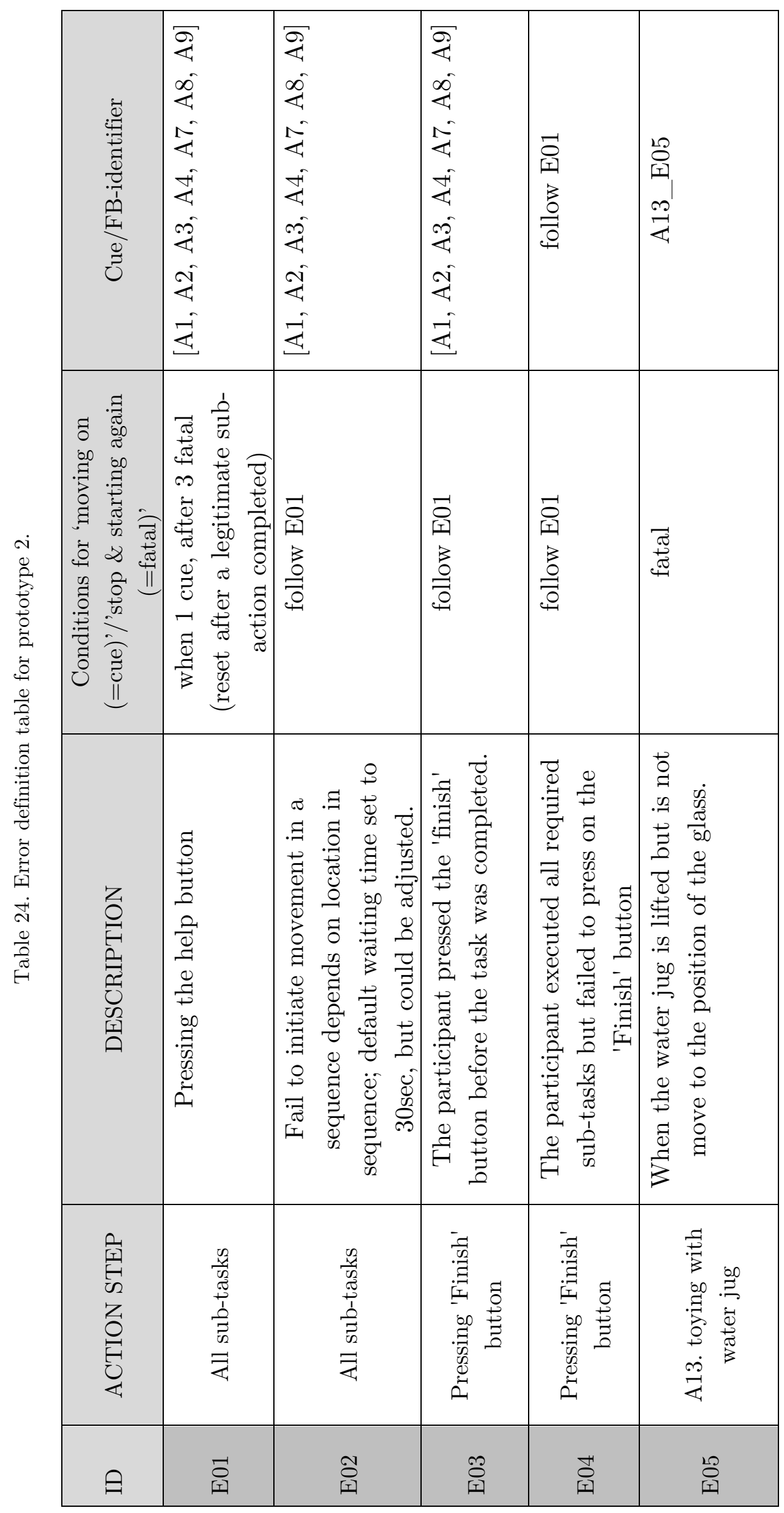




\begin{tabular}{|c|c|c|c|c|c|}
\hline 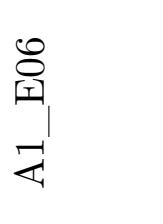 & 㐏 & 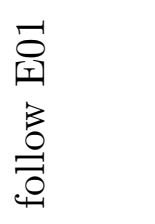 & 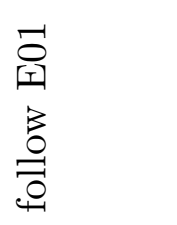 & 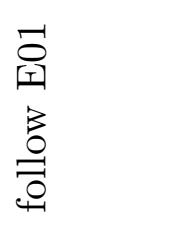 & $\begin{array}{l}\stackrel{\sim}{ت} \\
\underset{\text { II }}{\sharp} \\
\underset{4}{4}\end{array}$ \\
\hline 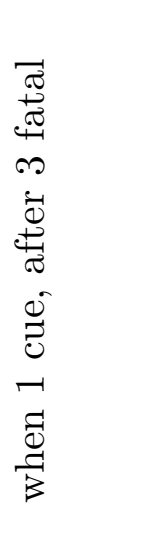 & 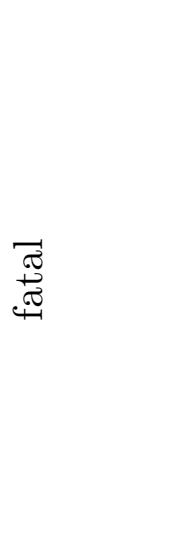 & 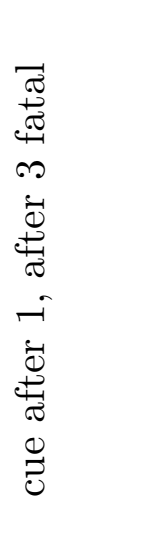 & 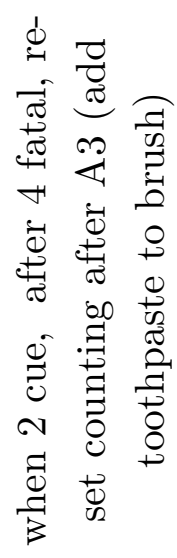 & 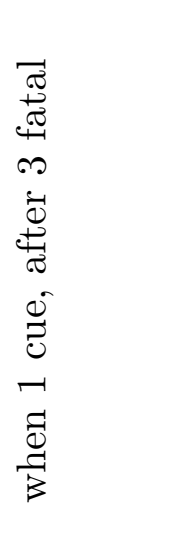 & 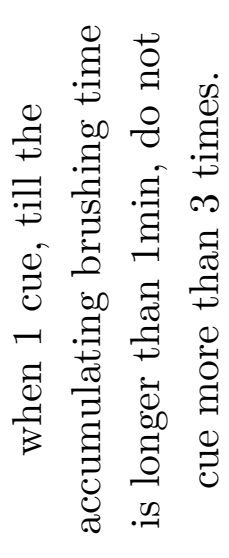 \\
\hline 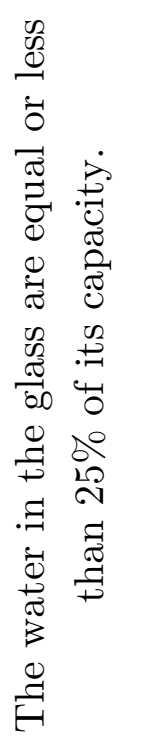 & 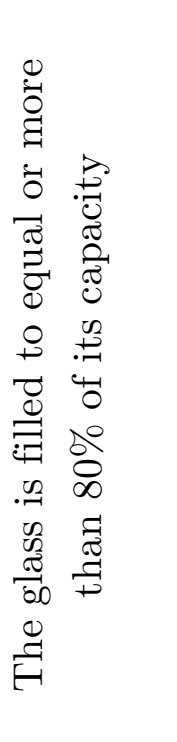 & 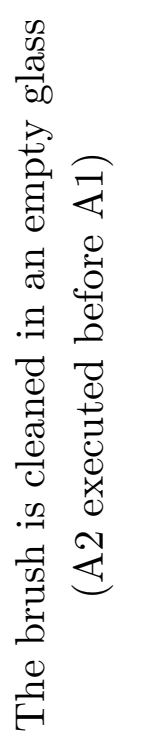 & 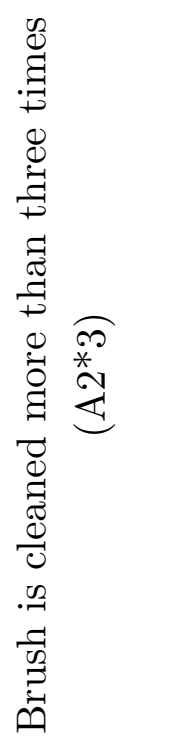 & 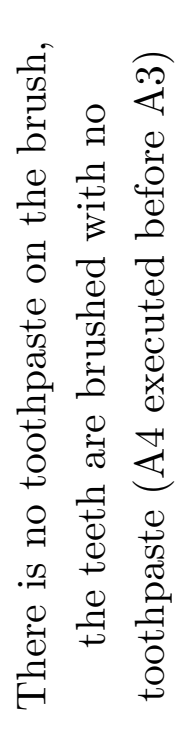 & 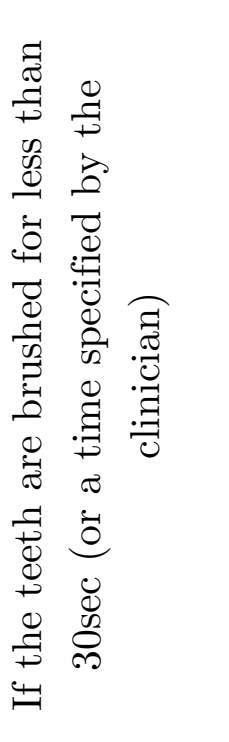 \\
\hline 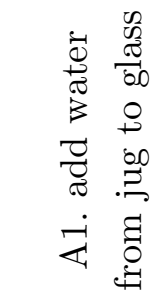 & 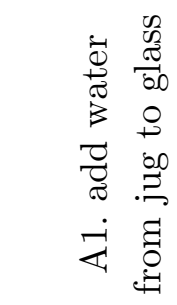 & 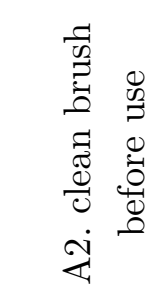 & 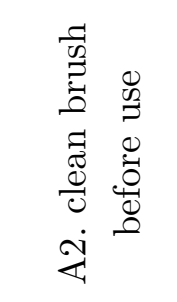 & 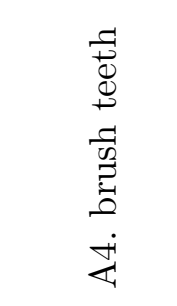 & 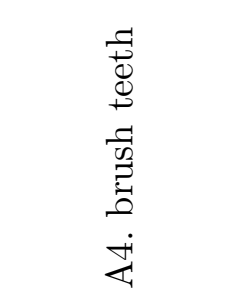 \\
\hline$\underset{\text { 足 }}{\varnothing}$ & 今o & 只 & $\stackrel{8}{8}$ & $\underset{\text { 뫼 }}{\stackrel{0}{2}}$ & $\underset{17}{\rightleftharpoons}$ \\
\hline
\end{tabular}




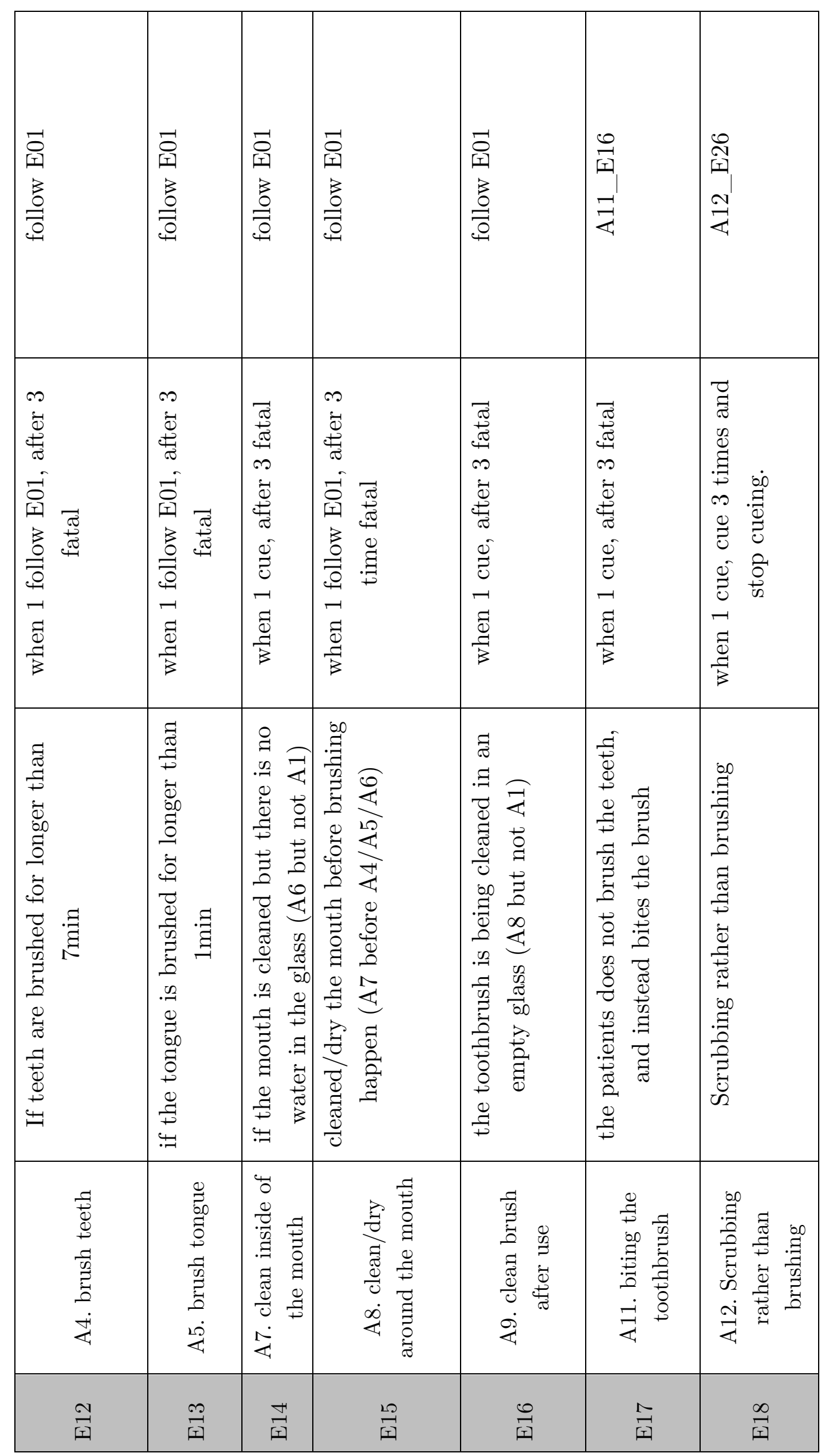




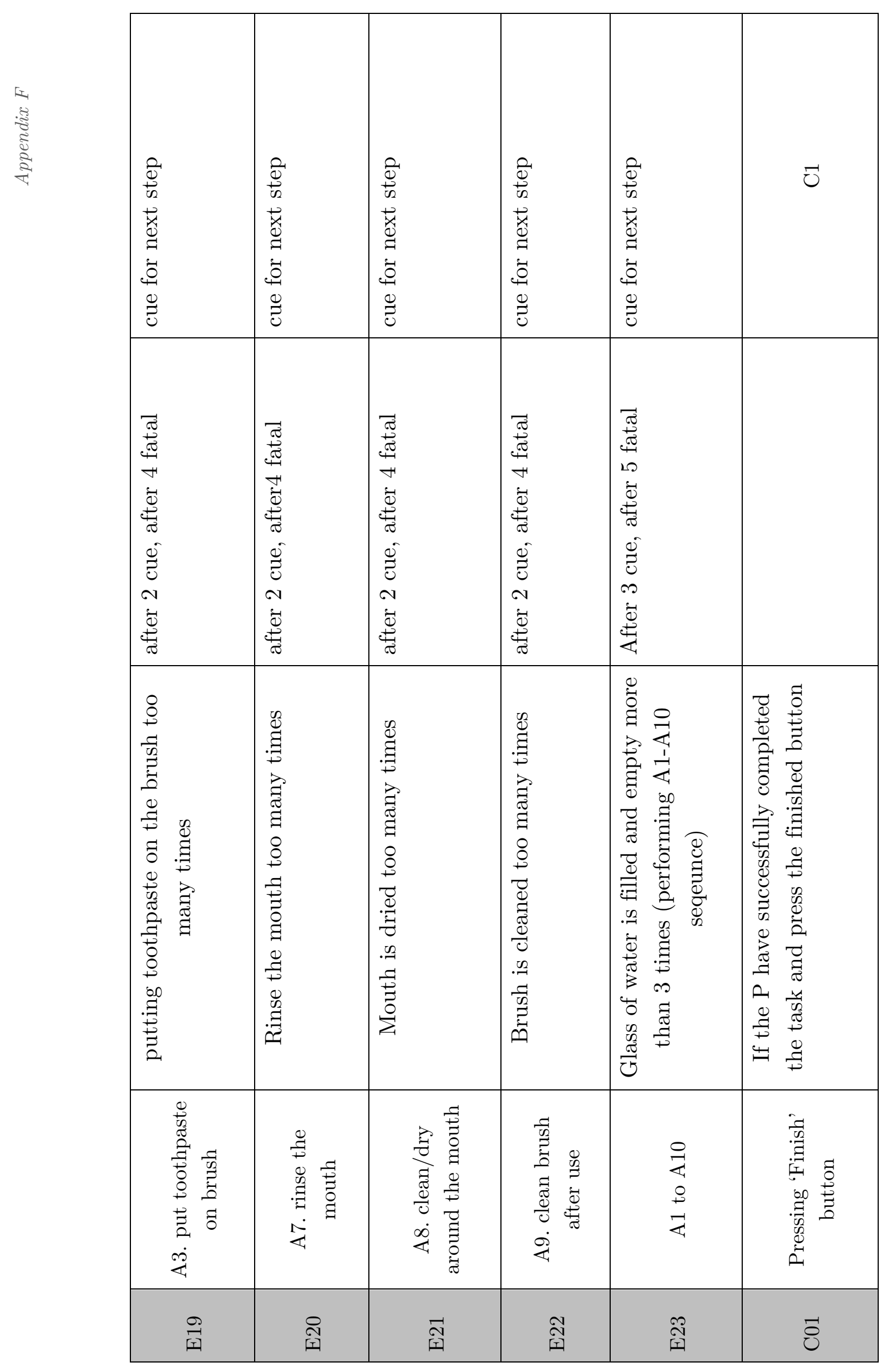





\section{LIST OF FIGURES}

Figure 1. Research framework proposed by S.T. March and G.F. Smith [14]29

Figure 2. Hugo Karl Liepmann, extracted from [22]....

Figure 3. Summarized scheme of brain regions mainly affected by the apraxia adapted from[24]. Notice that the shadow areas refer to the potentially critical areas affected and it does not necessary mean that the whole area is affected, instead there are not founded grounds to delimitate the affected area.

Figure 4. Introduction to the problematic of modelling; a simple task, pouring coffee into a vacuum flask. .47

Figure 5. Use of task models in the design cycle from [84] .......................50

Figure 6. MVC architecture schema from [85] ....................................50

Figure 7. La Machine Animale (a) Cover, (b) sketch up of the study of human biomechanics and (c) sketch up of the study of birds in flight [95]...53

Figure 8. TOTE example of hammering a nail re-described from [117]........58

Figure 9. Actor-critic architecture and pathways for supervisor information from [138]. .64

Figure 10. Example of a simple HTA for the task of changing a bulb. ........71

Figure 11. PGM defined from HTA shown in Figure 10.........................74

Figure 12. State machine cueing flow diagram.....................................78

Figure 13. MDP module - Profile module interactions. ..............................83

Figure 14. General three level architecture.......................................... 83

Figure 15. Description of the inner logic. .............................................. 84

Figure 16. Connection diagram. .................................................... 87

Figure 17. Inputs and outputs diagram............................................. 87

Figure 18. Decrement of times $[\mathrm{ms}]$ for each sample. ............................. 89

Figure 19.Increment of load in time. .............................................. 89

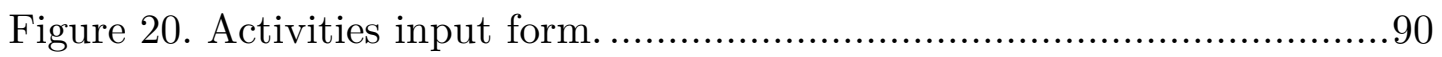

Figure 21.Creation of a probabilistic graphical tree. ............................... 91

Figure 22. State definition.................................................................91

Figure 23.Error activities...................................................................99

Figure 24.Error list generated. .........................................................92

Figure 25. Profile module interface...........................................................93 
Figure 26. Cue structure definition. 93

Figure 27. Model for improving attention postulated by M. Solhberg and C.

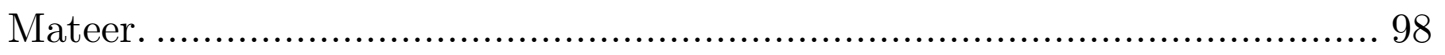

Figure 28. General architecture of the task model-cueing system............... 99

Figure 29. Interface snap shoot of the cue designer................................101

Figure 30. Proposed architecture for prospective cueing.......................... 103

Figure 31. Interface layouts for additional assitive information, cue progress and cue goal. .105

Figure 32. Experiment methodology for learning how to put in order a numbers puzzle and play a song with a piano. 106

Figure 33. Correct disposition of the puzzle. 107

Figure 34. Piano's keys used to play the song Jingle Bells in Do. ...............108

Figure 35. A still image indicates the correct piece to move......................109

Figure 36. Still image indicating which note to play. ..............................109

Figure 37. Results for piano test................................................ 111

Figure 38. Results for sliding puzzle test. ..................................... 111

Figure 39. The figure shows the modular methodology used with the Kinect ${ }^{\text {TM }}$ for data analysis. First, the data is acquired so as to launch the camera sensor using Kinect SDK@ functions and the interface developed, second, the data is filtered and synchronized, then the segmentation in epochs is carried out to represent and analyse the data. Finally, the information is stored. ..117 Figure 40. HTA for tea making. .119

Figure 41. This figure shows the object layout for the experiment. The objects involved. A cup of tea, a kettle, a tea bag; the sensor KinectTM and the Zebris system. The general Cartesian reference system is also represented.

Figure 42. Result of filtering Kinect ${ }^{\mathrm{TM}}$ data with a Butterworth low pass filter.

Figure 43. Comparison of position data for the Kinect (blue lines) and the Zebris system (green lines) from a single tea making task trial for the control (a) and the apraxic. .123

Figure 44. Correlation map between axes for the control participant (left panel) and the apraxic participant (right panel). .125

Figure 45. A volunteer doing the test.......................................... 128

Figure 46. NIBP module from the company RGB. 132 
Figure 47. NIBP interface. 132

Figure 48. Client- sub system from CogWatch architecture..................... 134

Figure 49. Appearance of the cue interface. ......................................... 138

Figure 50. Layout for tea preparation. .............................................. 140

Figure 51. Layout for toothbursihing. .......................................... 141

Figure 52. The quality step of technical validation. ............................. 142

Figure 53. German version of recipe-books...................................... 146

Figure 54. Crossover design for prototype I experiment.......................... 147

Figure 55. Number of successful teas made by patients in the six stages of the study. ... 149

Figure 56. Time spent by each patient brushing their teeth across the three trials.

Figure 57. Results from UoB efficacy trial. ......................................... 153

Figure 58. Reaction Time Meter ..................................................... 173

Figure 59. Position of the user and distance reference............................ 174

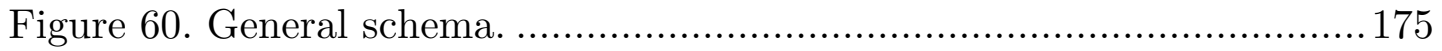

Figure 61 . Layout of the circuit.................................................... 176

Figure 62. Teardown of NIBP module............................................. 177 



\section{LIST OF TABLES}

Table 1. General stroke classification adapted from [16] ...........................34

Table 2. Different manifestations of apraxia from [23] ........................... 37

Table 3. Summary of error taxonomy, from [42]. "Examples are drawn from the task of preparing a sandwich, snack and drink and packing the three items into a lunch box, as used with various patient groups by M.F. Schwartz et al. [39], [40] and L.J. Buxbaum et al. [41]"

Table 4 . Therbligs, along with their mnemonic symbols and standard colours for charting adapted from [105] .55

Table 5. Error types and associated Psychological Mechanisms from [121] ...59

Table 6. List of required tools for changing a bulb...................................71

Table 7 . List of activities for changing a bulb.................................... 72

Table 8. Results of preliminary evaluation...................................... 79

Table 9. Available sequence for tea making. ........................................ 80

Table 10. Set of actions in closest sub-string problem............................ 81

Table 11. Mean Square Error (MSE) and cross-correlation values for the transverse, sagittal, and coronal axes................................................. 124

Table 12. Results of Reaction Time. .............................................. 130

Table 13. Comparison of means for visual and auditive RT.................... 130

Table 14. Devices for CogWatch prototypes. ......................................... 135

Table 15. Successful test percentage (\%) with prototype. .......................... 148

Table 16. Successful test percentage (\%) with recipe book........................ 148

Table 17. Order of the sequences performed by each patient; shadowed in red the steps performed after cueing...................................................... 151

Table 18. Average values of NIBP experiment..................................... 153

Table 19. Test Results for timing test channel 1.................................... 168

Table 20.Test Results for timing test channel 2 .................................... 171

Table 21. Action definition Prototype 1.......................................... 180

Table 22. Error definition table for prototype 1................................... 182

Table 23.Action definition Prototype 2 .............................................. 186

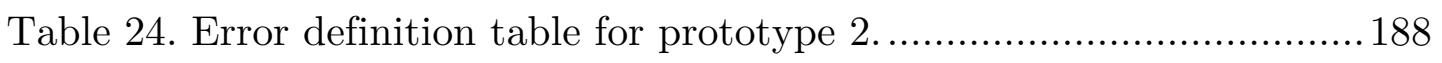





\section{BIBLIOGRAPHY}

[1] J. Ellul, La technique: ou L'enjeu du siècle. Paris: Économica, 1990.

[2] 'Welcome to COGWATCH'. [Online]. Available: http://www.cogwatch.eu/. [Accessed: 24-Sep-2015].

[3] M. Wooldridge and N. R. Jennings, 'Intelligent agents: theory and practice', Knowl. Eng. Rev., vol. 10, no. 2, pp. 115-152, Jun. 1995.

[4] C. Castelfranchi, 'Guarantees for autonomy in cognitive agent architecture', in Intelligent Agents, vol. 890, M. J. Wooldridge and N. R. Jennings, Eds. Berlin, Heidelberg: Springer Berlin Heidelberg, 1995, pp. $56-70$.

[5] M. R. Genesereth and S. P. Ketchpel, 'Software agents', Commun ACM, vol. 37, no. 7, pp. 48-53, 1994.

[6] K. Hartmann, G. Goldenberg, M. Daumüller, and J. Hermsdörfer, 'It takes the whole brain to make a cup of coffee: the neuropsychology of naturalistic actions involving technical devices', Neuropsychologia, vol. 43, no. 4, pp. 625-637, Jan. 2005.

[7] M. Ghallab, D. Nau, and P. Traverso, Automated Planning: Theory and Practice. 2004.

[8] J. M. Bradshaw, Ed., Software agents. Menlo Park, Calif. : Cambridge, Mass: AAAI Press; MIT Press, 1997.

[9] C. Hernández, R. Sanz, J. Gómez-Ramirez, L. S. Smith, A. Hussain, A. Chella, and I. Aleksander, Eds., From Brains to Systems, vol. 718. New York, NY: Springer New York, 2011.

[10] 'Mind Children - Hans Moravec | Harvard University Press'. [Online]. Available:

http://www.hup.harvard.edu/catalog.php?isbn=9780674576186.

[Accessed: 29-Sep-2015].

[11] J. C. R. Licklider, 'Man-computer symbiosis', Hum. Factors Electron. IRE Trans. On, no. 1, pp. 4-11, 1960.

[12] B. W. Genet N, 'Home care across Europe. Current structure and future challenges', Genet N, Boerma W, Kroneman M, Hutchinson A, Saltman $R B . \quad$ [Online]. Available: http://www.opimec.org/documentacion/2794/home-care-across-europecurrent-structure-and-future-challenges/. [Accessed: 05-Mar-2014].

[13] S. Kartakis, V. Sakkalis, P. Tourlakis, G. Zacharioudakis, and C. Stephanidis, 'Enhancing Health Care Delivery through Ambient 
Intelligence Applications', Sensors, vol. 12, no. 9, pp. 11435-11450, Aug. 2012.

[14] S. T. March and G. F. Smith, 'Design and Natural Science Research on Information Technology', Decis Support Syst, vol. 15, no. 4, pp. 251266, Dec. 1995.

[15] 'Types of Stroke - NHLBI, NIH'. [Online]. Available: http://www.nhlbi.nih.gov/health/health-topics/topics/stroke/types. [Accessed: 19-Aug-2016].

[16] L. A. Weisberg, R. L. Strub, and C. A. García, 'Fundamentos de neurología clínica / León A. Weisberg, Richard L. Strub, Carlos A. García', ResearchGate.

[17] S. Rasquin, J. Lodder, R. Ponds, I. Winkens, J. Jolles, and F. R. Verhey, 'Cognitive functioning after stroke: a one-year follow-up study', Dement. Geriatr. Cogn. Disord., vol. 18, no. 2, pp. 138-144, 2004.

[18] L. J. G. Rothi and K. M. Heilman, Apraxia: The Neuropsychology of Action. Psychology Press, 2014.

[19] J. M. S. Pearce, 'Hugo Karl Liepmann and apraxia', Clin. Med., vol. 9, no. 5, pp. 466-470, Jan. 2009.

[20] 'Drei Aufsätze aus dem Apraxiegebiet - Karger Publishers'. [Online]. Available: https://www.karger.com/Book/Home/219979. [Accessed: 10Sep-2015].

[21] H. Liepmann and O. Maas, 'A case of left-sided agraphia and apraxia in right-sided paralysis.', J. Psychol. Neurol., vol. 10, pp. 214-225, 1908.

[22] G. Goldenberg, Apraxia: The Cognitive Side of Motor Control. OUP Oxford, 2013.

[23] K. M. Heilman, 'APRAXIA':, Contin. Lifelong Learn. Neurol., vol. 16, pp. 86-108, Aug. 2010.

[24] G. Goldenberg, 'Apraxia - Agreements and controversies', Cortex, vol. 57, pp. 306-308, Aug. 2014.

[25] K. Morady and G. W. Humphreys, 'Comparing action disorganization syndrome and dual-task load on normal performance in everyday action tasks', Neurocase, vol. 15, no. 1, pp. 1-12, Jan. 2009.

[26] M. F. Schwartz, E. S. Reed, M. Montgomery, C. Palmer, and N. H. Mayer, 'The quantitative description of action disorganisation after brain damage: A case study', Cogn. Neuropsychol., vol. 8, no. 5, pp. 381-414, 1991.

[27] S. Monsell and J. Driver, Control of Cognitive Processes: Attention and Performance XVIII. MIT Press, 2000. 
[28] G. W. Humphreys and E. M. E. Forde, 'Disordered action schema and action disorganisation syndrome', Cogn. Neuropsychol., vol. 15, no. 6, pp. 771-812, 1998.

[29] M. J. Riddoch and G. W. Humphreys, BORB: Birmingham object recognition battery. LEA, 1993.

[30] J. Decety, Perception and Action: Recent Advances in Cognitive Neuropsychology. Psychology Press, 1998.

[31] K. Morady and G. Humphreys, 'Multiple task demands in action disorganization syndrome', Neurocase, vol. 17, no. 5, pp. 461-472, Oct. 2011.

[32] N. Smania, S. M. Aglioti, F. Girardi, M. Tinazzi, A. Fiaschi, A. Cosentino, and E. Corato, 'Rehabilitation of limb apraxia improves daily life activities in patients with stroke', Neurology, vol. 67, no. 11, pp. 2050-2052, 2006.

[33] C. M. van Heugten, J. Dekker, B. G. Deelman, A. J. van Dijk, F. C. Stehmann-Saris, and A. Kinebanian, 'Measuring disabilities in stroke patients with apraxia: A validation study of an observational method', Neuropsychol. Rehabil., vol. 10, no. 4, pp. 401-414, 2000.

[34] S. Giaquinto, S. Buzzelli, L. Francesco, A. Lottarini, P. Montenero, P. T. In, and G. Nolfe, 'On the prognosis of outcome after stroke', Acta Neurol. Scand., vol. 100, no. 3, pp. 202-208, 1999.

[35] B. Hanna-Pladdy, K. M. Heilman, and A. L. Foundas, 'Ecological implications of ideomotor apraxia Evidence from physical activities of daily living', Neurology, vol. 60, no. 3, pp. 487-490, 2003.

[36] F. I. Mahoney, 'Functional evaluation: the Barthel index.', Md. State Med. J., vol. 14, pp. 61-65, 1965.

[37] F. M. Nouri and N. B. Lincoln, 'An extended activities of daily living scale for stroke patients', Clin. Rehabil., vol. 1, no. 4, pp. 301-305, 1987.

[38] W.-L. Bickerton, M. J. Riddoch, D. Samson, A. B. Balani, B. Mistry, and G. W. Humphreys, 'Systematic assessment of apraxia and functional predictions from the Birmingham Cognitive Screen', J. Neurol. Neurosurg. Psychiatry, vol. 83, no. 5, pp. 513-521, 2012.

[39] M. F. Schwartz, M. W. Montgomery, L. J. Buxbaum, S. S. Lee, T. G. Carew, H. Coslett, M. Ferraro, E. Fitzpatrick-DeSalme, T. Hart, and N. Mayer, 'Naturalistic action impairment in closed head injury.', Neuropsychology, vol. 12, no. 1, p. 13, 1998.

[40] M. F. Schwartz, L. J. Buxbaum, M. W. Montgomery, E. FitzpatrickDeSalme, T. Hart, M. Ferraro, S. S. Lee, and H. B. Coslett, 
'Naturalistic action production following right hemisphere stroke', Neuropsychologia, vol. 37, no. 1, pp. 51-66, 1998.

[41] L. J. Buxbaum, 'Ideational apraxia and naturalistic action', Cogn. Neuropsychol., vol. 15, no. 6-8, pp. 617-643, 1998.

[42] R. P. Cooper, M. F. Schwartz, P. Yule, and T. Shallice, 'The simulation of action disorganisation in complex activities of daily living', Cogn. Neuropsychol., vol. 22, no. 8, pp. 959-1004, 2005.

[43] R. P. Cooper, 'Tool use and related errors in ideational apraxia: The quantitative simulation of patient error profiles', Cortex, vol. 43, no. 3, pp. 319-337, 2007.

[44] M. F. Schwartz, M. W. Montgomery, E. J. Fitzpatrick-DeSalme, C. Ochipa, H. B. Coslett, and N. H. Mayer, 'Analysis of a disorder of everyday action', Cogn. Neuropsychol., vol. 12, no. 8, pp. 863-892, 1995.

[45] M. F. Schwartz, M. W. Montgomery, L. J. Buxbaum, S. S. Lee, T. G. Carew, H. B. Coslett, M. Ferraro, E. Fitzpatrick-DeSalme, T. Hart, and N. Mayer, 'Naturalistic action impairment in closed head injury.', Neuropsychology, vol. 12, no. 1, pp. 13-28, 1998.

[46] J. Díaz-Guzmán, J. A. Egido-Herrero, B. Fuentes, C. Fernández-Pérez, R. Gabriel-Sánchez, G. Barbera, and S. Abilleira, 'Incidencia de ictus en España: estudio Iberictus. Datos del estudio piloto', Rev. Neurol., vol. 48, no. 2, pp. 61-65, 2009.

[47] J. Díaz-Guzmán, J.-A. Egido, R. Gabriel-Sánchez, G. Barberá-Comes, B. Fuentes-Gimeno, and C. Fernández-Pérez, 'Stroke and transient ischemic attack incidence rate in Spain: the IBERICTUS study', Cerebrovasc. Dis., vol. 34, no. 4, pp. 272-281, 2012.

[48] 'WHO | World Health Organization'. [Online]. Available: http://www.who.int/en/. [Accessed: 25-Jun-2014].

[49] A. G. Thrift, D. A. Cadilhac, T. Thayabaranathan, G. Howard, V. J. Howard, P. M. Rothwell, and G. A. Donnan, 'Global stroke statistics', Int. J. Stroke Off. J. Int. Stroke Soc., vol. 9, no. 1, pp. 6-18, Jan. 2014.

[50] 'World Stroke Organization - Home'. [Online]. Available: http://www.world-stroke.org/. [Accessed: 05-Jun-2013].

[51] K. D. Cicerone, C. Dahlberg, K. Kalmar, D. M. Langenbahn, J. F. Malec, T. F. Bergquist, T. Felicetti, J. T. Giacino, J. P. Harley, D. E. Harrington, J. Herzog, S. Kneipp, L. Laatsch, and P. A. Morse, 'Evidence-based cognitive rehabilitation: recommendations for clinical practice', Arch. Phys. Med. Rehabil., vol. 81, no. 12, pp. 1596-1615, Dec. 2000. 
[52] K. D. Cicerone, C. Dahlberg, J. F. Malec, D. M. Langenbahn, T. Felicetti, S. Kneipp, W. Ellmo, K. Kalmar, J. T. Giacino, J. P. Harley, L. Laatsch, P. A. Morse, and J. Catanese, 'Evidence-Based Cognitive Rehabilitation: Updated Review of the Literature From 1998 Through 2002', Arch. Phys. Med. Rehabil., vol. 86, no. 8, pp. 1681-1692, Aug. 2005 .

[53] E. Frank Lopresti, A. Mihailidis, and N. Kirsch, 'Assistive technology for cognitive rehabilitation: State of the art', Neuropsychol. Rehabil., vol. 14, no. 1-2, pp. 5-39, 2004.

[54] A. M. Seelye, M. Schmitter-Edgecombe, B. Das, and D. J. Cook, 'Application of Cognitive Rehabilitation Theory to the Development of Smart Prompting Technologies', Biomed. Eng. IEEE Rev. In, vol. 5, pp. 29-44, 2012.

[55] V. C. Georgopoulos and G. A. Malandraki, 'A Fuzzy Cognitive Map Hierarchical Model for Differential Diagnosis of Dysarthrias and Apraxia of Speech', in Engineering in Medicine and Biology Society, 2005. IEEE-EMBS 2005. 27th Annual International Conference of the, 2005, pp. 2409-2412.

[56] E. M. Albornoz, L. D. Vignolo, C. E. Martinez, and D. H. Milone, 'Genetic wrapper approach for automatic diagnosis of speech disorders related to Autism', in 2013 IEEE 14th International Symposium on Computational Intelligence and Informatics (CINTI), 2013, pp. 387392.

[57] H. Poizner, J. Lancaster, E. Tunik, S. Narayana, C. Franklin, W. Rogers, X. Li, P. T. Fox, and D. A. Robin, 'Towards a Healthy Human Model of Neural Disorders of Movement', IEEE Trans. Neural Syst. Rehabil. Eng., vol. 20, no. 6, pp. 853-857, Nov. 2012.

[58] B. Brewer, S. McDowell, and L. Worthen-Chaudhari, 'Poststroke Upper Extremity Rehabilitation: A Review of Robotic Systems and Clinical Results', Top. Stroke Rehabil., vol. 14, no. 6, pp. 22-44, Jan. 2007.

[59] 'Paro Therapeutic Robot'. [Online]. Available: http://www.parorobots.com/. [Accessed: 06-Mar-2014].

[60] C. Giraldo, S. Helal, and W. Mann, 'mPCA-A mobile patient caregiving assistant for Alzheimer patients', in First International Workshop on Ubiquitous Computing for Cognitive Aids (UbiCog'02), 2002.

[61] T. Gentry, J. Wallace, C. Kvarfordt, and K. B. Lynch, 'Personal digital assistants as cognitive aids for high school students with autism: results 
of a community-based trial', J. Vocat. Rehabil., vol. 32, no. 2, pp. 101$107,2010$.

[62] S. Helal, C. Giraldo, Y. Kaddoura, C. Lee, H. El Zabadani, and W. Mann, 'Smart phone based cognitive assistant', in UbiHealth 2003: The 2nd International Workshop on Ubiquitous Computing for Pervasive Healthcare Applications, 2003.

[63] B. Das, A. M. Seelye, B. L. Thomas, D. J. Cook, L. B. Holder, and M. Schmitter-Edgecombe, 'Using smart phones for context-aware prompting in smart environments', in 2012 IEEE Consumer Communications and Networking Conference (CCNC), 2012, pp. 399403.

[64] J.-A. Lozano, J.-A. Gil-Gomez, M. Alcaniz, J. Chirivella, and J. Ferri, 'Activities of daily living in a virtual reality system for cognitive rehabilitation', in Virtual Rehabilitation International Conference, 2009, 2009, pp. 205-205.

[65] E. Klinger, A. Kadri, J. L. Le Guiet, P. Coignard, N. D. Lac, P. A. Joseph, E. Sorita, P. Fuchs, L. Leroy, and F. Servant, 'AGATHE: A tool for personalized rehabilitation of cognitive functions', in 2013 International Conference on Virtual Rehabilitation (ICVR), 2013, pp. 214-215.

[66] E. Klinger, A. Kadri, E. Sorita, J.-L. Le Guiet, P. Coignard, P. Fuchs, L. Leroy, N. du Lac, F. Servant, and P.-A. Joseph, 'AGATHE: A tool for personalized rehabilitation of cognitive functions based on simulated activities of daily living', IRBM, vol. 34, no. 2, pp. 113-118, Apr. 2013.

[67] F. D. Rose, B. M. Brooks, and A. A. Rizzo, 'Virtual Reality in Brain Damage Rehabilitation: Review', Cyberpsychol. Behav., vol. 8, no. 3, pp. 241-262, Jun. 2005.

[68] 'IATSL - Intelligent Assistive Technology and Systems Lab'. [Online]. Available: http://www.ot.utoronto.ca/iatsl/. [Accessed: 06-Mar-2014].

[69] 'ISAAC System Home Page'. [Online]. Available: http://www.cosys.us/index.htm. [Accessed: 06-Mar-2014].

[70] P. Gorman, R. Dayle, C.-A. Hood, and L. Rumrell, 'Effectiveness of the ISAAC cognitive prosthetic system for improving rehabilitation outcomes with neurofunctional impairment', NeuroRehabilitation, vol. 18, no. 1, pp. 57-67, 2003.

[71] R. A. Powell and H. M. Single, 'Focus groups', Int. J. Qual. Health Care, vol. 8, no. 5, pp. 499-504, 1996.

[72] K. Boehner, J. Vertesi, P. Sengers, and P. Dourish, 'How HCI interprets the probes', in Proceedings of the SIGCHI conference on Human factors in computing systems, 2007, pp. 1077-1086. 
[73] S. M. Shafer, H. J. Smith, and J. C. Linder, 'The power of business models', Bus. Horiz., vol. 48, no. 3, pp. 199-207, 2005.

[74] N. Souchon, Q. Limbourg, and J. Vanderdonckt, 'Task Modelling in Multiple Contexts of Use', in Interactive Systems:Design, Specification, and Verification, P. Forbrig, Q. Limbourg, J. Vanderdonckt, and B. Urban, Eds. Springer Berlin Heidelberg, 2002, pp. 59-73.

[75] S. Gui and L. Luo, 'Reliability Analysis of Task Model in Real-Time Fault-Tolerant Systems', 2012, pp. 368-374.

[76] J. Arróspide Laborda and L. Salgado Álvarez de Sotomayor, 'On-road visual vehicle tracking using Markov chain Monte Carlo with metropolis sampling', Int. J. Automot. Technol., vol. 13, no. 6, pp. 955-961, Oct. 2012.

[77] X. Koutsoukos, F. Zhao, H. Haussecker, J. Reich, and P. Cheung, 'Fault modeling for monitoring and diagnosis of sensor-rich hybrid systems', in Decision and Control, 2001. Proceedings of the 40th IEEE Conference on, 2001, vol. 1, pp. 793-801.

[78] S. Li, 'A model-based fault detection and diagnostic methodology for secondary HVAC systems', Drexel University, 2009.

[79] M. Beetz, M. Tenorth, D. Jain, and J. Bandouch, 'Towards automated models of activities of daily life', Technol. Disabil., vol. 22, no. 1, p. 27, 2010.

[80] Y. Kuniyoshi, M. Inaba, and H. Inoue, 'Learning by watching: Extracting reusable task knowledge from visual observation of human performance', Robot. Autom. IEEE Trans. On, vol. 10, no. 6, pp. 799$822,1994$.

[81] J. M. Carmena, M. A. Lebedev, R. E. Crist, J. E. O’Doherty, D. M. Santucci, D. F. Dimitrov, P. G. Patil, C. S. Henriquez, and M. A. L. Nicolelis, 'Learning to Control a Brain-Machine Interface for Reaching and Grasping by Primates', PLoS Biol., vol. 1, no. 2, p. e2, 2003.

[82] A. Gharsellaoui, Y. Bellik, and C. Jacquet, 'A Run Time Executable Task Model for Ambient Intelligent Environments', 2013, pp. 691-696.

[83] 'Task Model - Model-based User Interfaces Incubator Group Wiki'. [Online]. Available: http://www.w3.org/2005/Incubator/model-basedui/wiki/Task_Model. [Accessed: 22-Sep-2015].

[84] 'design-cycle.jpg (Imagen JPEG, $733 \times 418$ píxeles) - Escalado (93\%)'. [Online]. Available: http://www.w3.org/2012/02/ctt/design-cycle.jpg. [Accessed: 23-Nov-2015].

[85] 'MVC Architecture - Google Chrome'. [Online]. Available: https://developer.chrome.com/apps/app_frameworks. [Accessed: 18May-2016]. 
[86] 'ASP.NET MVC Overview'. [Online]. Available: https://msdn.microsoft.com/en-us/library/dd381412(v=vs.108).aspx. [Accessed: 18-May-2016].

[87] E. L. Lehmann, 'A theory of some multiple decision problems, I', Ann. Math. Stat., pp. 1-25, 1957.

[88] E. L. Lehmann, 'A theory of some multiple decision problems. II', Ann. Math. Stat., pp. 547-572, 1957.

[89] T. J. Stewart, 'A critical survey on the status of multiple criteria decision making theory and practice', Omega, vol. 20, no. 5, pp. 569586, 1992.

[90] S. D. Pohekar and M. Ramachandran, 'Application of multi-criteria decision making to sustainable energy planning - a review', Renew. Sustain. Energy Rev., vol. 8, no. 4, pp. 365-381, 2004.

[91] L. Ljung, System identification - Theory for the User. Prentice-Hall, 1999 .

[92] J. Von Neumann and O. Morgenstern, Theory of games and economic behavior. Princeton university press, 2007.

[93] J. F. Nash Jr, 'The bargaining problem', Econom. J. Econom. Soc., pp. 155-162, 1950.

[94] J. M. Smith, Evolution and the Theory of Games. Cambridge university press, 1982.

[95] E.-J. Marey, La machine animale, locomotion terrestre et aérienne. Paris, G. Baillie, 1873.

[96] E. Muybridge, W. D. Marks, H. Allen, and F. X. Dercum, Animal locomotion: the Muybridge work at the University of Pennsylvania. JB Lippincott Company, 1888.

[97] M. L. Latash and V. M. Zatsiorsky, Classics in Movement Science. Human Kinetics, 2001.

[98] A. D. Kuo, J. M. Donelan, and A. Ruina, 'Energetic consequences of walking like an inverted pendulum: step-to-step transitions', Exerc. Sport Sci. Rev., vol. 33, no. 2, pp. 88-97, 2005.

[99] C. J. Walsh, K. Endo, and H. Herr, 'A quasi-passive leg exoskeleton for load-carrying augmentation', Int. J. Humanoid Robot., vol. 4, no. 3, pp. 487-506, 2007.

[100] N. Aphiratsakun, K. Chairungsarpsook, and M. Parnichkun, 'ZMP based gait generation of AIT's Leg Exoskeleton', in Computer and Automation Engineering (ICCAE), 2010 The 2nd International Conference on, 2010, vol. 5, pp. 886-890.

[101] 'Muybridge'. [Online]. Available: http://www.muybridge.org. [Accessed: 11-Nov-2015]. 
[102] F. W. Taylor, The principles of scientific management. 1911. Dover Publications, MineoIa, Nueva York, 1998.

[103] J. R. Hackman and G. R. Oldham, 'Motivation through the design of work: Test of a theory', Organ. Behav. Hum. Perform., vol. 16, no. 2, pp. 250-279, 1976.

[104] L. M. Gilbreth, 'The psychology of management', Ind. Eng. Eng. Dig. May June 1912, 1914.

[105] 'The Gilbreth Network: Therbligs'. [Online]. Available: http://gilbrethnetwork.tripod.com/therbligs.html. [Accessed: 11-Nov2015].

[106] F. B. Gilbreth, Bricklaying system. The MC Clark publishing co., 1909.

[107] F. B. Gilbreth and E. G. Carey, Cheaper by the Dozen. Open Road Media, 2013.

[108] E. F. Moore, 'Gedanken Experiments on Sequential Machines', 1956.

[109] G. H. Mealy, 'A Method for Synthesizing Sequential Circuits', Bell Syst. Tech. J., vol. 34, 1955.

[110] '07-SeqLogicIII.ppt - 07-SeqLogicIIIx2.pdf'. [Online]. Available: https://inst.eecs.berkeley.edu/ cs150/fa05/Lectures/07-

SeqLogicIIIx2.pdf. [Accessed: 13-Nov-2015].

[111] C. Reutenauer, The mathematics of Petri nets. Prentice-Hall, Inc., 1990.

[112] A. Knutilla, C. Schlenoff, S. Ray, S. T. Polyak, A. Tate, S. C. Cheah, and R. C. Anderson, Process specification language: An analysis of existing representations. US Department of Commerce, Technology Administration, National Institute of Standards and Technology, 1998.

[113] J. Rumbaugh, I. Jacobson, and G. Booch, Unified Modeling Language Reference Manual, The (2Nd Edition). Pearson Higher Education, 2004.

[114] M. Grüninger, 'Ontology of the Process Specification Language.', Handb. Ontol., vol. 1, 2004.

[115] S. A. White, 'Business process modeling notation', Specif. BPMI Org, 2004.

[116] G. A. Miller, E. Galanter, and K. H. Pribram, Plans and the Structure of Behavior. Adams Bannister Cox, 1986.

[117] G. Ward and R. Morris, 'Introduction to the psychology of planning', Cogn. Psychol. Plan., pp. 1-34, 2005.

[118] J. Annett and K. D. Duncan, 'Task analysis and training design.', 1967.

[119] E. Hollnagel, Handbook of Cognitive Task Design. CRC Press, 2003. 
[120] N. A. Stanton, 'Hierarchical task analysis: Developments, applications, and extensions', Appl. Ergon., vol. 37, no. 1, pp. 55-79, Jan. 2006.

[121] A. Sears and J. A. Jacko, The Human-Computer Interaction Handbook: Fundamentals, Evolving Technologies and Emerging Applications, Second Edition. CRC Press, 2007.

[122] D. E. Embrey, 'SHERPA: A systematic human error reduction and prediction approach', 1986.

[123] N. A. Stanton and C. Baber, 'Validating task analysis for error identification: reliability and validity of a human error prediction technique', Ergonomics, vol. 48, no. 9, pp. 1097-1113, 2005.

[124] M. L. Puterman, Markov decision processes: discrete stochastic dynamic programming. John Wiley \& Sons, 2014.

[125] G. E. Monahan, 'State of the art-a survey of partially observable Markov decision processes: theory, models, and algorithms', Manag. Sci., vol. 28, no. 1, pp. 1-16, 1982.

[126] R. S. Sutton and A. G. Barto, Reinforcement learning: An introduction, vol. 1. MIT press Cambridge, 1998.

[127] F. L. Lewis and D. Vrabie, 'Reinforcement learning and adaptive dynamic programming for feedback control', Circuits Syst. Mag. IEEE, vol. 9, no. 3, pp. 32-50, 2009.

[128] A. G. Barto, R. S. Sutton, and C. J. Watkins, 'Sequential decision problems and neural networks', in Advances in neural information processing systems, 1990, pp. 686-693.

[129] L. P. Kaelbling, M. L. Littman, and A. W. Moore, 'Reinforcement learning: A survey', J. Artif. Intell. Res., pp. 237-285, 1996.

[130] D. P. Bertsekas and J. N. Tsitsiklis, 'Neuro-dynamic programming: an overview', in Decision and Control, 1995., Proceedings of the 34th IEEE Conference on, 1995, vol. 1, pp. 560-564.

[131] C. Szepesvári, 'Algorithms for reinforcement learning', Synth. Lect. Artif. Intell. Mach. Learn., vol. 4, no. 1, pp. 1-103, 2010.

[132] H. Van Hasselt, 'Reinforcement learning in continuous state and action spaces', in Reinforcement learning, Springer, 2012, pp. 207-251.

[133] E. Levin, R. Pieraccini, and W. Eckert, 'A stochastic model of humanmachine interaction for learning dialog strategies', Speech Audio Process. IEEE Trans. On, vol. 8, no. 1, pp. 11-23, 2000.

[134] R. S. Sutton, 'Learning to predict by the methods of temporal differences', Mach. Learn., vol. 3, no. 1, pp. 9-44, 1988.

[135] G. Tesauro, 'Temporal difference learning of backgammon strategy', in Proceedings of the Ninth International Workshop on Machine Learning, ML92, 2014, pp. 451-457. 
[136] C. J. Watkins and P. Dayan, 'Q-learning', Mach. Learn., vol. 8, no. 34, pp. 279-292, 1992.

[137] I. Grondman, L. Buşoniu, G. A. Lopes, and R. Babuška, 'A survey of actor-critic reinforcement learning: Standard and natural policy gradients', Syst. Man Cybern. Part C Appl. Rev. IEEE Trans. On, vol. 42, no. 6, pp. 1291-1307, 2012.

[138] M. T. Rosenstein and A. G. Barto, 'Supervised Actor-Critic Reinforcement Learning', Handb. Learn. Approx. Dyn. Program., vol. 2, p. 359, 2004.

[139] D. Embrey, C. Blackett, P. Marsden, and J. Peachey, 'Development of a human cognitive workload assessment tool', MCA Final Rep., 2006.

[140] K. A. Ericsson and A. C. Lehmann, 'Expert and exceptional performance: evidence of maximal adaptation to task constraints', Annu. Rev. Psychol., vol. 47, pp. 273-305, 1996.

[141] E. Gibney, 'Google AI algorithm masters ancient game of Go', Nat. News, vol. 529, no. 7587, p. 445, Jan. 2016.

[142] R. Clark, 'Cognitive Task Analysis for Expert-Based Instruction in Healthcare', in Handbook of Research on Educational Communications and Technology, J. M. Spector, M. D. Merrill, J. Elen, and M. J. Bishop, Eds. Springer New York, 2014, pp. 541-551.

[143] V. Venkataraman, P. Turaga, N. Lehrer, M. Baran, T. Rikakis, and S. L. Wolf, 'Decision support for stroke rehabilitation therapy via describable attribute-based decision trees', in 2014 36th Annual International Conference of the IEEE Engineering in Medicine and Biology Society, 2014, pp. 3154-3159.

[144] E. M. D. Jean-Baptiste, P. Rotshtein, and M. Russell, 'POMDP Based Action Planning and Human Error Detection', in Artificial Intelligence Applications and Innovations, vol. 458, R. Chbeir, Y. Manolopoulos, I. Maglogiannis, and R. Alhajj, Eds. Cham: Springer International Publishing, 2015, pp. 250-265.

[145] J. Hoey, P. Poupart, C. Boutilier, and A. Mihailidis, 'POMDP models for assistive technology', in Proc. AAAI Fall Symposium on Caring Machines: AI in Eldercare, 2005.

[146] Y.-C. Tsai, 'Kinempt: a Kinect-based prompting system to transition autonomously through vocational tasks for individuals with cognitive impairments', in Proceedings of the 14th international ACM SIGACCESS conference on Computers and accessibility, 2012, pp. 299300.

[147] F. J. Damerau, 'A technique for computer detection and correction of spelling errors', Commun. ACM, vol. 7, no. 3, pp. 171-176, 1964. 
[148] V. I. Levenshtein, 'Binary codes capable of correcting deletions, insertions, and reversals', in Soviet physics doklady, 1966, vol. 10, pp. $707-710$.

[149] R. Hilliard, 'ISO/IEC/IEEE 42010 Homepage', ISO/IEC/IEEE 42010 Homepage. [Online]. Available: http://www.isoarchitecture.org/42010/. [Accessed: 18-May-2016].

[150] 'Extensible Markup Language (XML)'. [Online]. Available: http://www.w3.org/XML/. [Accessed: 02-Dec-2015].

[151] 'How to: Encrypt XML Elements with Asymmetric Keys'. [Online]. Available: https://msdn.microsoft.com/enus/library/ms229746(v=vs.110).aspx. [Accessed: 02-Dec-2015].

[152] A. Jacobs, 'The pathologies of big data', Commun. ACM, vol. 52, no. 8, pp. 36-44, 2009.

[153] R. Herold, Managing an information security and privacy awareness and training program. CRC press, 2010.

[154] P. E. Pailing, S. J. Segalowitz, and P. L. Davies, 'Speed of responding and the likelihood of error-like activity in correct trial ERPs', in Psychophysiology, 2000, vol. 37, pp. S76-S76.

[155] M. Falkenstein, J. Hohnsbein, J. Hoormann, and L. Blanke, 'Effects of errors in choice reaction tasks on the ERP under focused and divided attention', Psychophysiological Brain Res., vol. 1, pp. 192-195, 1990.

[156] C. S. Carter, T. S. Braver, D. M. Barch, M. M. Botvinick, D. Noll, and J. D. Cohen, 'Anterior cingulate cortex, error detection, and the online monitoring of performance', Science, vol. 280, no. 5364, pp. 747-749, 1998.

[157] N. Yeung, M. M. Botvinick, and J. D. Cohen, 'The neural basis of error detection: conflict monitoring and the error-related negativity.', Psychol. Rev., vol. 111, no. 4, p. 931, 2004.

[158] R. S Mahesh, Handbook of Research on Global Information Technology Management in the Digital Economy. IGI Global, 2008.

[159] B. F. Skinner, Science and human behavior. Simon and Schuster, 1965.

[160] B. F. Skinner, About behaviorism. Vintage, 2011.

[161] S. Kornblum, T. Hasbroucq, and A. Osman, 'Dimensional overlap: cognitive basis for stimulus-response compatibility-a model and taxonomy.', Psychol. Rev., vol. 97, no. 2, p. 253, 1990.

[162] S. Harnad, 'Categorical Perception', in Encyclopedia of Cognitive Science, vol. LXVII, MacMillan: Nature Publishing Group, 2003.

[163] V. K. Emery, P. J. Edwards, J. A. Jacko, K. P. Moloney, L. Barnard, T. Kongnakorn, F. Sainfort, and I. U. Scott, 'Toward achieving universal usability for older adults through multimodal feedback', in 
ACM SIGCAPH Computers and the Physically Handicapped, 2003, pp. $46-53$.

[164] J. A. Jacko, I. U. Scott, F. Sainfort, L. Barnard, P. J. Edwards, V. K. Emery, T. Kongnakorn, K. P. Moloney, and B. S. Zorich, 'Older adults and visual impairment: what do exposure times and accuracy tell us about performance gains associated with multimodal feedback?', in Proceedings of the SIGCHI conference on Human factors in computing systems, 2003, pp. 33-40.

[165] J. M. M. Gas, M. L. H. Serrano, M. V. Junyent, and A. C. Mas, 'Análisis de los componentes del tiempo de reacción', Anu. Psicol. UB J. Psychol., no. 65, pp. 139-152, 1995.

[166] I. S. MacKenzie, 'Fitts' law as a research and design tool in humancomputer interaction', Hum.-Comput. Interact., vol. 7, no. 1, pp. 91139, 1992.

[167] A. Newell and P. S. Rosenbloom, 'Mechanisms of skill acquisition and the law of practice', Cogn. Ski. Their Acquis., vol. 1.

[168] H. Cen, K. Koedinger, and B. Junker, 'Learning factors analysis-a general method for cognitive model evaluation and improvement', in Intelligent tutoring systems, 2006, pp. 164-175.

[169] M. M. Sohlberg and C. A. Mateer, 'Improving attention and managing attentional problems', Ann. N. Y. Acad. Sci., vol. 931, no. 1, pp. 359$375,2001$.

[170] J. Yamato, J. Ohya, and K. Ishii, 'Recognizing human action in timesequential images using hidden markov model', in Computer Vision and Pattern Recognition, 1992. Proceedings CVPR'92., 1992 IEEE Computer Society Conference on, 1992, pp. 379-385.

[171] P. Scovanner, S. Ali, and M. Shah, 'A 3-dimensional sift descriptor and its application to action recognition', in Proceedings of the 15th international conference on Multimedia, 2007, pp. 357-360.

[172] H. Jhuang, T. Serre, L. Wolf, and T. Poggio, 'A biologically inspired system for action recognition', in Computer Vision, 200\%. ICCV $200 \%$. IEEE 11th International Conference on, 2007, pp. 1-8.

[173] M. D. Rodriguez, J. Ahmed, and M. Shah, 'Action mach a spatiotemporal maximum average correlation height filter for action recognition', in Computer Vision and Pattern Recognition, 2008. CVPR 2008. IEEE Conference on, 2008, pp. 1-8.

[174] H. Wang, A. Kläser, C. Schmid, and C.-L. Liu, 'Action recognition by dense trajectories', in Computer Vision and Pattern Recognition (CVPR), 2011 IEEE Conference on, 2011, pp. 3169-3176. 
[175] S. Ji, W. Xu, M. Yang, and K. Yu, '3D convolutional neural networks for human action recognition', Pattern Anal. Mach. Intell. IEEE Trans. On, vol. 35, no. 1, pp. 221-231, 2013.

[176] J. C. Bezdek, Pattern recognition with fuzzy objective function algorithms. Springer Science \& Business Media, 2013.

[177] C. Schüldt, I. Laptev, and B. Caputo, 'Recognizing human actions: a local SVM approach', in Pattern Recognition, 2004. ICPR 2004. Proceedings of the 17th International Conference on, 2004, vol. 3, pp. $32-36$.

[178] 'Non-invasive user monitoring in daily tasks for cognitive rehabilitation - Archivo Digital UPM'. [Online]. Available: http://oa.upm.es/38573/. [Accessed: 02-Dec-2015].

[179] 'Support', META. [Online]. Available: http://meta.watch/pages/support. [Accessed: 02-Dec-2015].

[180] R. M. Schafer, 'Acoustic space', in Dwelling, place and environment, Springer, 1985, pp. 87-98.

[181] M. S. Armstrong and L. Orseau, 'Safely interruptible agents', 2016.

[182] D. D. Wackerly, W. Mendenhall, and R. L. Scheaffer, Mathematical statistics with applications, 7th ed. Belmont, CA: Thomson Brooks/Cole, 2008.

[183] 'The Comprehensive $\mathrm{R}$ Archive Network'. [Online]. Available: https://cran.r-project.org/. [Accessed: 18-Feb-2016].

[184] J. Russell and R. Cohn, McNemar's Test. Book on Demand, 2012.

[185] 'zebris Medical GmbH CMS-HS Measuring System for 3D Real Time Motion Analysis' [Online]. Available: http://zebrismedical.de/english/medizin/medizin-3dmesssysteme-cms-hsl-cmshs.php?navanchor=1010053. [Accessed: 04-Dec-2015].

[186] H. M. Overhoff, D. Lazovic, M. Liebing, and C. Macher, 'Total knee arthroplasty: coordinate system definition and planning based on 3-D ultrasound image volumes', in International Congress Series, 2001, vol. 1230, pp. 292-299.

[187] R. Cooper and T. Shallice, 'Contention Scheduling and the control of routine activities', Cogn. Neuropsychol., vol. 17, no. 4, pp. 297-338, Jun. 2000.

[188] B. Coley, B. M. Jolles, A. Farron, A. Bourgeois, F. Nussbaumer, C. Pichonnaz, and K. Aminian, 'Outcome evaluation in shoulder surgery using 3D kinematics sensors', Gait Posture, vol. 25, no. 4, pp. 523-532, 2007.

[189] J. Cohen, Statistical power analysis for the behavioral sciences. Academic press, 2013. 
[190] X. H. Vidal, 'Las pruebas diagnósticas y los parámetros que las definen', Med.-Programa Form. Médica Contin. Acreditado, vol. 8, no. 118, pp. 6318-6327, 2003.

[191] G. G. Gil, J. T. Cano, V. L. Caudet, P. P. Campo, A. Meléndez, and A. Carrillo, 'Influencia del color en el tiempo de reacción', 2004.

[192] Ó. Martínez de Quel Pérez, 'El tiempo de reacción visual en el kárate', Ciencias, 2003.

[193] J. P. Tejero and J. J. Rojo-González, 'Estudio del tiempo de reacción ante estímulos sonoros y visuales', Mot. Rev. Cienc. Act. Física Deporte, no. 27, pp. 149-162, 2011.

[194] 'IBM Watson Health'. [Online]. Available: http://www.ibm.com/smarterplanet/us/en/ibmwatson/health/. [Accessed: 03-Dec-2015].

[195] National Center for Chronic Disease Prevention, The Power of Prevention: Chronic Disease... The Public Health Challenge of the 21st Century (Atlanta, GA: Centers for Disease Control and Prevention, 2009). 2012.

[196] A. F. Members, J. Perk, G. D. Backer, H. Gohlke, I. Graham, Ž. Reiner, M. Verschuren, C. Albus, P. Benlian, G. Boysen, R. Cifkova, C. Deaton, S. Ebrahim, M. Fisher, G. Germano, R. Hobbs, A. Hoes, S. Karadeniz, A. Mezzani, E. Prescott, L. Ryden, M. Scherer, M. Syvänne, W. J. M. S. O. Reimer, C. Vrints, D. Wood, J. L. Zamorano, F. Zannad, O. experts who contributed to parts of the Guidelines, M. T. Cooney, E. C. for P. G. (cpg), J. Bax, H. Baumgartner, C. Ceconi, V. Dean, C. Deaton, R. Fagard, C. Funck-Brentano, D. Hasdai, A. Hoes, P. Kirchhof, J. Knuuti, P. Kolh, T. McDonagh, C. Moulin, B. A. Popescu, Ž. Reiner, U. Sechtem, P. A. Sirnes, M. Tendera, A. Torbicki, A. Vahanian, S. Windecker, D. Reviewers, C. Funck-Brentano, P. A. Sirnes, V. Aboyans, E. A. Ezquerra, C. Baigent, C. Brotons, G. Burell, A. Ceriello, J. D. Sutter, J. Deckers, S. D. Prato, H.-C. Diener, D. Fitzsimons, Z. Fras, R. Hambrecht, P. Jankowski, U. Keil, M. Kirby, M. L. Larsen, G. Mancia, A. J. Manolis, J. McMurray, A. Pająk, A. Parkhomenko, L. Rallidis, F. Rigo, E. Rocha, L. M. Ruilope, E. van der Velde, D. Vanuzzo, M. Viigimaa, M. Volpe, O. Wiklund, and C. Wolpert, 'European Guidelines on cardiovascular disease prevention in clinical practice (version 2012)', Eur. Heart J., p. ehs092, May 2012.

[197] G. J. Palmer, M. G. Ziegler, and C. R. Lake, 'Response of norepinephrine and blood pressure to stress increases with age', $J$. Gerontol., vol. 33, no. 4, pp. 482-487, 1978. 
[198] R. B. Devereux, T. G. Pickering, G. A. Harshfield, H. D. Kleinert, L. Denby, L. Clark, D. Pregibon, M. Jason, B. Kleiner, and J. S. Borer, 'Left ventricular hypertrophy in patients with hypertension: importance of blood pressure response to regularly recurring stress.', Circulation, vol. 68, no. 3, pp. 470-476, 1983.

[199] J. Brod, V. Fencl, Z. Hejl, and J. Jirka, 'Circulatory changes underlying blood pressure elevation during acute emotional stress (mental arithmetic) in normotensive and hypertensive subjects.', Clin. Sci., vol. 18, pp. 269-279, 1959.

[200] K. A. Matthews, K. L. Woodall, and M. T. Allen, 'Cardiovascular reactivity to stress predicts future blood pressure status.', Hypertension, vol. 22, no. 4, pp. 479-485, 1993.

[201] P. J. Gianaros, S. W. Derbtshire, J. C. May, G. J. Siegle, M. A. Gamalo, and J. R. Jennings, 'Anterior cingulate activity correlates with blood pressure during stress', Psychophysiology, vol. 42, no. 6, pp. 627$635,2005$.

[202] A. G. Fisher, 'The assessment of IADL motor skills: an application of many-faceted Rasch analysis', Am. J. Occup. Ther., vol. 47, no. 4, pp. 319-329, 1993.

[203] Q. McNemar, 'Note on the sampling error of the difference between correlated proportions or percentages', Psychometrika, vol. 12, no. 2, pp. 153-157, Jun. 1947.

[204] S. A. Kauffman, The Origins of Order: Self Organization and Selection in Evolution. Oxford University Press, 1993.

[205] E. W. Weisstein, 'Lorenz Attractor'. [Online]. Available: http://mathworld.wolfram.com/LorenzAttractor.html. [Accessed: 12Nov-2015]. 